\title{
Appalachian Basin Bituminous Coal: Sulfur Content and Potential Sulfur Dioxide Emissions of Coal Mined for Electrical Power Generation
}

By Michael H. Trippi, Leslie F. Ruppert, Emil D. Attanasi, Robert C. Milici, and

Philip A. Freeman

Chapter G.5 of

Coal and Petroleum Resources in the Appalachian Basin:

Distribution, Geologic Framework, and Geochemical Character

Edited by Leslie F. Ruppert and Robert T. Ryder

Professional Paper 1708 
Suggested citation:

Trippi, M.H., Ruppert, L.F., Attanasi, E.D., Milici, R.C., and Freeman, P.A., 2014, Appalachian basin bituminous coal; Sulfur content and potential sulfur dioxide emissions of coal mined for electrical power generation, chap. G.5 of Ruppert, L.F., and Ryder, R.T., eds., Coal and petroleum resources in the Appalachian basin; Distribution, geologic framework, and geochemical character: U.S. Geological Survey Professional Paper 1708, 68 p., http://dx.doi. org/10.3133/pp1708G.5. 


\section{Contents}

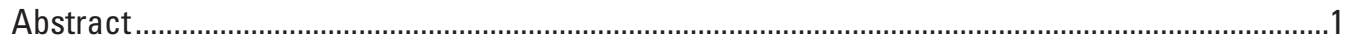

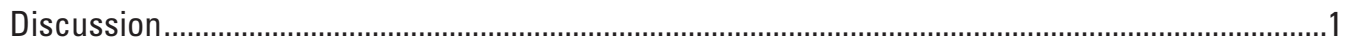

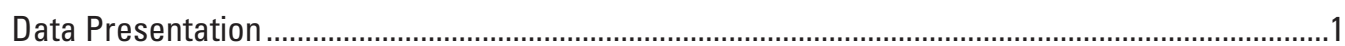

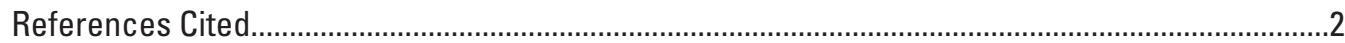

\section{Figures}

1-4. Maps showing-

1. Weighted average sulfur content of bituminous coal that was mined in the Appalachian basin for electrical power generation for 1983 through 1995.

2. Weighted average sulfur content of bituminous coal that was mined in the Appalachian basin for electrical power generation for 1996 through 2005

3. Potential $\mathrm{SO}_{2}$ emissions of bituminous coal that was mined in the Appalachian basin for electrical power generation for 1983 through 1995

4. Potential $\mathrm{SO}_{2}$ emissions of bituminous coal that was mined in the Appalachian basin for electrical power generation for 1996 through 2005 .

5-13. Graphs showing-

5. Amount of bituminous coal that was mined for electrical power generation from six Appalachian basin counties that exhibited generally increasing production trends

6. Amount of bituminous coal that was mined for electrical power generation from two Appalachian basin counties that exhibited generally decreasing production trends.

7. Amount of bituminous coal that was mined for electrical power generation from six Appalachian basin counties that exhibited fluctuating production trends

8. Sulfur content of bituminous coal that was mined for electrical power generation from six Appalachian basin counties that exhibited generally increasing production trends

9. Sulfur content of bituminous coal that was mined for electrical power generation from two Appalachian basin counties that exhibited generally decreasing production trends

10. Sulfur content of bituminous coal that was mined for electrical power generation from six Appalachian basin counties that exhibited fluctuating production trends

11. Potential $\mathrm{SO}_{2}$ emissions based on sulfur percentages of coal that was mined for electrical power generation from six Appalachian basin counties that exhibited generally increasing production trends

12. Potential $\mathrm{SO}_{2}$ emissions based on sulfur percentages of coal that was mined for electrical power generation from two Appalachian basin counties that exhibited generally decreasing production trends 
13. Potential $\mathrm{SO}_{2}$ emissions based on sulfur percentages of coal that was mined for electrical power generation from six Appalachian basin counties that exhibited fluctuating production trends.

\section{Tables}

1. Total weight of bituminous coal that was mined in the Appalachian basin for electrical power generation.

2. Heating values of bituminous coal that was mined in the Appalachian basin for electrical power generation.

3. Weighted average sulfur content of bituminous coal that was mined in the Appalachian basin for electrical power generation

4. Potential $\mathrm{SO}_{2}$ emissions of bituminous coal that was mined in the Appalachian basin for electrical power generation

5. Summary statistics comparing weighted average sulfur content and potential $\mathrm{SO}_{2}$ emissions for 1983 through 1995 and 1996 through 2005

\section{Conversion Factors}

\begin{tabular}{lcl}
\hline \multicolumn{1}{c}{ Multiply } & By & \multicolumn{1}{c}{ To obtain } \\
\hline & Mass & \\
\hline ton, short $(2,000 \mathrm{lb})$ & 0.9072 & megagram $(\mathrm{Mg})$ \\
\hline & Energy & \\
\hline $\begin{array}{l}\text { British thermal unit }(\mathrm{Btu}) \\
\text { British thermal unit per pound } \\
(\text { Btu/lb) }\end{array}$ & $1,055.056$ & joule $(\mathrm{J})$ \\
\hline
\end{tabular}




\title{
Appalachian Basin Bituminous Coal: Sulfur Content and Potential Sulfur Dioxide Emissions of Coal Mined for Electrical Power Generation
}

\author{
By Michael H. Trippi, ${ }^{1}$ Leslie F. Ruppert, ${ }^{1}$ Emil D. Attanasi, ${ }^{1}$ Robert C. Milici, ${ }^{1}$ and Philip A. Freeman'
}

\begin{abstract}
Data from 157 counties in the Appalachian basin of average sulfur content of coal mined for electrical power generation from 1983 through 2005 show a general decrease in the number of counties where coal mining has occurred and a decrease in the number of counties where higher sulfur coals $\left(>2\right.$ percent sulfur) were mined. Calculated potential $\mathrm{SO}_{2}$ emissions (assuming no post-combustion $\mathrm{SO}_{2}$ removal) show a corresponding decrease over the same period of time.
\end{abstract}

\section{Discussion}

The purpose of this report is to present trends in average sulfur content and calculated potential sulfur dioxide $\left(\mathrm{SO}_{2}\right)$ emission (assuming no post-combustion $\mathrm{SO}_{2}$ removal) levels of coal mined for electrical power generation from 1983 through 2005 in the Appalachian basin. During this time, the U.S. Congress passed several rules and regulations that set upper limits on $\mathrm{SO}_{2}$ emissions from coal-burning powerplants. In 1990, the Congress set a national ceiling of 9.5 million tons of $\mathrm{SO}_{2}$ emissions per year from electrical power generation plants with the passing of Phase II of the 1990 Clean Air Act Amendments (Public Law 101-549) (U.S. Environmental Protection Agency, 2008). In 2005, the U.S. Environmental Protection Agency declared a new ceiling for annual $\mathrm{SO}_{2}$ emissions from coal-fired power plants for 28 States and the District of Columbia with the adoption of the Clean Air Interstate Rule (CAIR) (U.S. Environmental Protection Agency, 2007). In 2008, a U.S. court ordered the Environmental Protection Agency to issue a new rule to implement the Clean Air Act requirements concerning the transport of air pollution across State lines, and the U.S. Environmental Protection Agency adapted the Cross-State Air Pollution Rule (CSAPR; U.S. Environmental Protection Agency, 2011). In 2014, the CSAPR was upheld by the U.S. Supreme Court. The new rule sets annual $\mathrm{SO}_{2}$ (and other emissions, including nitrogen

${ }^{1}$ U.S. Geological Survey, Reston, Va. dioxide and particulate matter) ceilings for the 28 States and the District of Columbia. Powerplant compliance may be achieved through the use of a combination of (1) initial $\mathrm{SO}_{2}$ emissions allowances; (2) purchase of additional emissions allowances from other powerplants; (3) use of low sulfur coal; and (4) reduction of sulfur using other techniques such as removal of sulfur-bearing minerals through washing and use of flue-gas desulfurization "scrubbers" during combustion. As of the end of 2005, there were still 6.2 million tons of $\mathrm{SO}_{2}$ emissions allowances banked by the industry as a result of overcompliance in the 1980s and 1990s (U.S. Environmental Protection Agency, 2006). Until this inventory is reduced, actual $\mathrm{SO}_{2}$ emissions will probably exceed the annual allocation to the industry.

This report is an update of a previous report by Attanasi and Milici (1998), who compiled data from the Federal Energy Regulatory Commission (FERC) and the Energy Information Administration (EIA) for 1983 through 1995. Their original data can be found in tables 1 through 3 of this report. Data for the years 1996 through 2005 were obtained from COALdat, a database (released in April 2006) of U.S. coal production and market transactions available by subscription from Platts, a division of The McGraw-Hill Companies, Inc. These original data can be found in tables 1 through 4 of the report. In addition, pounds of $\mathrm{SO}_{2}$ per million British thermal units ( $\mathrm{lb} \mathrm{SO}_{2} / \mathrm{MMBtu}$ ) were calculated for the years 1983 through 1995 , and are shown in table 4.

Data in this report represent average values of coal mined for electrical power generation in each county and should not be used to infer detailed spatial and stratigraphic distribution of the sulfur content and $\mathrm{SO}_{2}$ emissions of the coal. Finally, it should be noted that in these data there is no way to distinguish between washed and unwashed coal.

\section{Data Presentation}

Comparison of the weighted average sulfur percentage maps for the years 1983 through 1995 (fig. 1; table 3) and 1996 through 2005 (fig. 2; table 3) shows a general decrease 
in the number of counties where coal mining has occurred and a decrease in the number of counties where higher sulfur coals ( $>2$ percent sulfur) were mined. During the period 1996 through 2005, 19.1 percent of the counties shown in figure 1 reported no mining production (table 5). Of the 127 counties that reported mining production between 1996 and 2005, 41.7 percent of the counties showed an increase in weight percent sulfur, 55.9 percent showed a decrease in weight percent sulfur, and 2.4 percent showed no change in weight percent sulfur. The vast majority of sulfur increases and decreases (93.5 percent) were one percentage point or less (table 5). Five counties showed increases in weight percent sulfur of greater than one percentage point (Guernsey and Washington Counties, Ohio; Mercer County, Pa.; Grundy County, Tenn.; and Ohio County, W. Va.). Mercer County, Pa., showed the greatest increase (4.21 percentage points) (table 5). Three counties showed decreases in weight percent sulfur of greater than one percentage point (Greenup and Wolfe Counties, Ky., and Mason County, W. Va.). Mason County, W. Va., showed the greatest decrease (2.12 percentage points) (table 5).

The decreases in sulfur percentages may be due to increased coal cleaning or increased mining of coal beds with lower sulfur content. Herhal and Minnucci (1991) report that 50 percent of all coal produced in the United States in 1983 was mechanically cleaned, including 73 percent of Central Appalachian basin coal, 79 percent of Southern Appalachian basin coal, and 70 percent of Northern Appalachian basin coal. Akers and others (1994) state that 77 percent of bituminous coal shipments from Eastern and Midwestern States was cleaned to some degree, and Laurila (1998) reports that 55 percent of coal produced in the United States was cleaned. Unfortunately, detailed data describing the amount of coal cleaned annually are not included in EIA, FERC, or Platts databases available to the public and are probably not collected by these or any other organizations. Likewise, data about coal beds mined are very sparse and can only be conjectured without detailed data availability.

Figures 3 and 4 and table 4 show weighted average pounds of $\mathrm{SO}_{2}$ per million British thermal units for Appalachian basin coal produced for electrical power generation for the periods 1983 to 1995 and 1996 to 2005, respectively. These maps exhibit trends similar to those observed in the percent sulfur maps for the corresponding time periods (figs. 1,2). Of the 127 counties that reported mining activity from 1996 to 2005, 45.7 percent of the counties showed an increase in pounds of $\mathrm{SO}_{2}$ per million British thermal units, 53.5 percent showed a decrease in pounds of $\mathrm{SO}_{2}$ per million British thermal units, and 0.8 percent of the counties showed no change in pounds of $\mathrm{SO}_{2}$ per million British thermal units (table 5). Most of the $\mathrm{SO}_{2}$ increases and decreases (81.7 percent) were less than one pound of $\mathrm{SO}_{2}$ per million British thermal units (table 5). Twelve counties showed increases of greater than 1 pound of $\mathrm{SO}_{2}$ per million British thermal units. Mercer County, Pa., had the greatest increase (8.18 $\mathrm{lb} \mathrm{SO}_{2} / \mathrm{MMBtu}$ ) (table 5). Eleven counties showed decreases of greater than 1 pound of $\mathrm{SO}_{2}$ per million British thermal units; Mason County, W. Va., had the greatest decrease (3.75 $\mathrm{lb} \mathrm{SO}_{2} / \mathrm{MMBtu}$ ) (table 5).

Between 1983 and 2005, 14 counties had peak annual coal production values greater than 10,000 thousand tons; these values are graphed by year in figures 5 through 7 . Of these counties, six exhibited increasing production (fig. 5), two exhibited decreasing production (fig. 6), and six exhibited fluctuating production (fig. 7). Variations in weight percent of sulfur for the same 14 counties remained relatively constant for 12 of the counties throughout the 23-year period (figs. 8-10). In Indiana County, Pa., the coal exhibited a slight increase in weight percent sulfur after 1998 (fig. 9), and in Marshall County, W. Va., the coal exhibited a decrease in weight percent sulfur after 1998 (fig. 10). Trends of pounds of $\mathrm{SO}_{2}$ per million British thermal units are similar to those observed for weight percent sulfur (figs. 11-13). Most counties had coal with relatively steady levels of $\mathrm{SO}_{2}$ except for Indiana County, $\mathrm{Pa}$., where the coal exhibited a slight increase in $\mathrm{SO}_{2}$ after 1998 (fig. 12) and Marshall County, W. Va., where the coal exhibited a large decrease in $\mathrm{SO}_{2}$ after 1998 (fig. 13). Coal mined in Marshall County, W. Va., is predominantly from the Pittsburgh bed, and has been cleaned for many years (Mitch Blake, West Virginia Geological and Economic Survey, written commun., 2007). We must therefore conclude that the decrease in sulfur in Marshall County, W. Va., must be due to mining of coal beds with lower sulfur content and (or) closing of mines in high sulfur coal beds. The decrease in sulfur content of coal mined in Marshall County, W. Va., as delivered to powerplants may be due to selective mining within Pittsburgh coal bed mines, mining of additional coal beds with lower sulfur content, and (or) closing of mines with higher sulfur content. Without detailed county-level data about coal cleaning and coal beds being mined, we can only speculate that decreases in weight percent sulfur and pounds of $\mathrm{SO}_{2}$ per million British thermal units within particular counties may be due to increased coal cleaning or increased mining of coal beds with lower sulfur content.

\section{References Cited}

Akers, D., Raleigh, C., Shirey, G., and Dospoy, R., 1994, The effect of coal cleaning on trace elements; Draft report, application of algorithms: Palo Alto, Calif., Electric Power Research Institute (EPRI). (Prepared for EPRI by CQ, Inc.)

Attanasi, E.D., and Milici, R.C., 1998, Appalachian basin bituminous coal; Weight percent sulfur of produced coal by county, as received at the powerplant; U.S. Geological Survey Open-File Report 98-763, available only online at http://pubs.usgs.gov/of/1998/of98-763/.

Herhal, A.J., and Minnucci, Chris, 1991, Project summary; Assessment of physical coal cleaning practices for 
sulfur removal: U.S. Environmental Protection Agency [Report] EPA/600/S7-90/013, 12 p. (Also available online from http://www.epa.gov/nscep/index.html; search for 600S790013.)

Laurila, Mel, 1998, Coal prep in the next millennium, in Keystone Coal Industry Manual: Chicago, Ill., Intertec Publishing Company, p. 258-267.

U.S. Environmental Protection Agency, 2006, Acid rain program; 2005 progress report: U.S. Environmental Protection Agency Web site at http://www.epa.gov/airmarket/progress/ docs/2005report.pdf. (Accessed July 7, 2008.)
U.S. Environmental Protection Agency, 2007, Clean Air Interstate Rule [CAIR]: U.S. Environmental Protection Agency Web site at http://www.epa.gov/cleanairinterstaterule. (Accessed July 7, 2008.)

U.S. Environmental Protection Agency, 2008, Clean Air Act: U.S. Environmental Protection Agency Web site at http://www.epa.gov/air/caa/. (Accessed July 7, 2008.)

U.S. Environmental Protection Agency, 2012, Cross-State Air Pollution Rule: U.S. Environmental Protection Agency Web page accessed May 13, 2014, at http://www.epa.gov/ airtransport/CSAPR/. 

Figures 1-13 


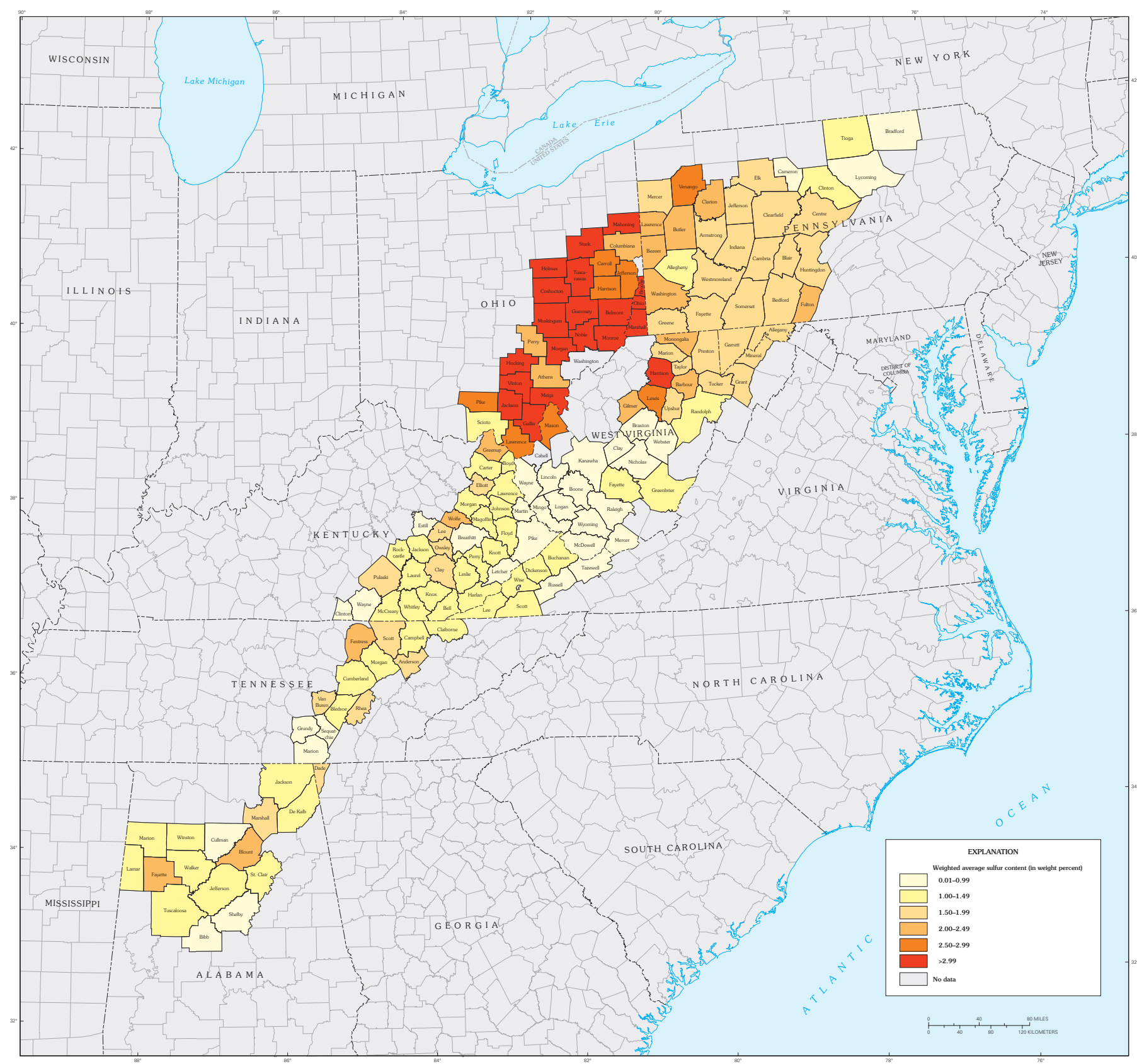

Figure 1. Weighted average sulfur content (in weight percent) of bituminous coal that was mined in the Appalachian basin for electrical power generation. Data are shown by county for 1983 through 1995 and are given in table 3. 


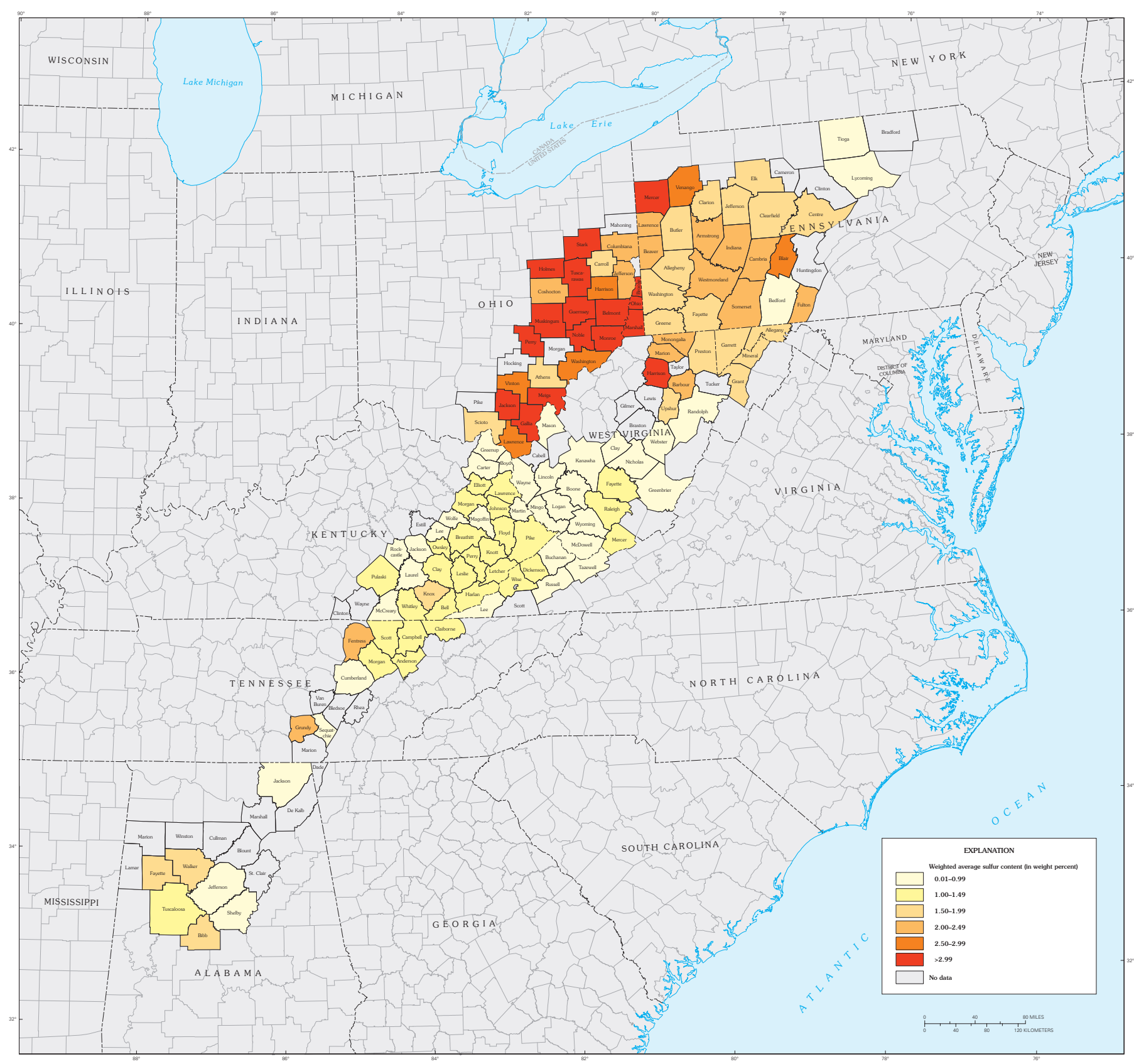

Figure 2. Weighted average sulfur content (in weight percent) of bituminous coal that was mined in the Appalachian basin for electrical power generation. Data are shown by county for 1996 through 2005 and are given in table 3. 


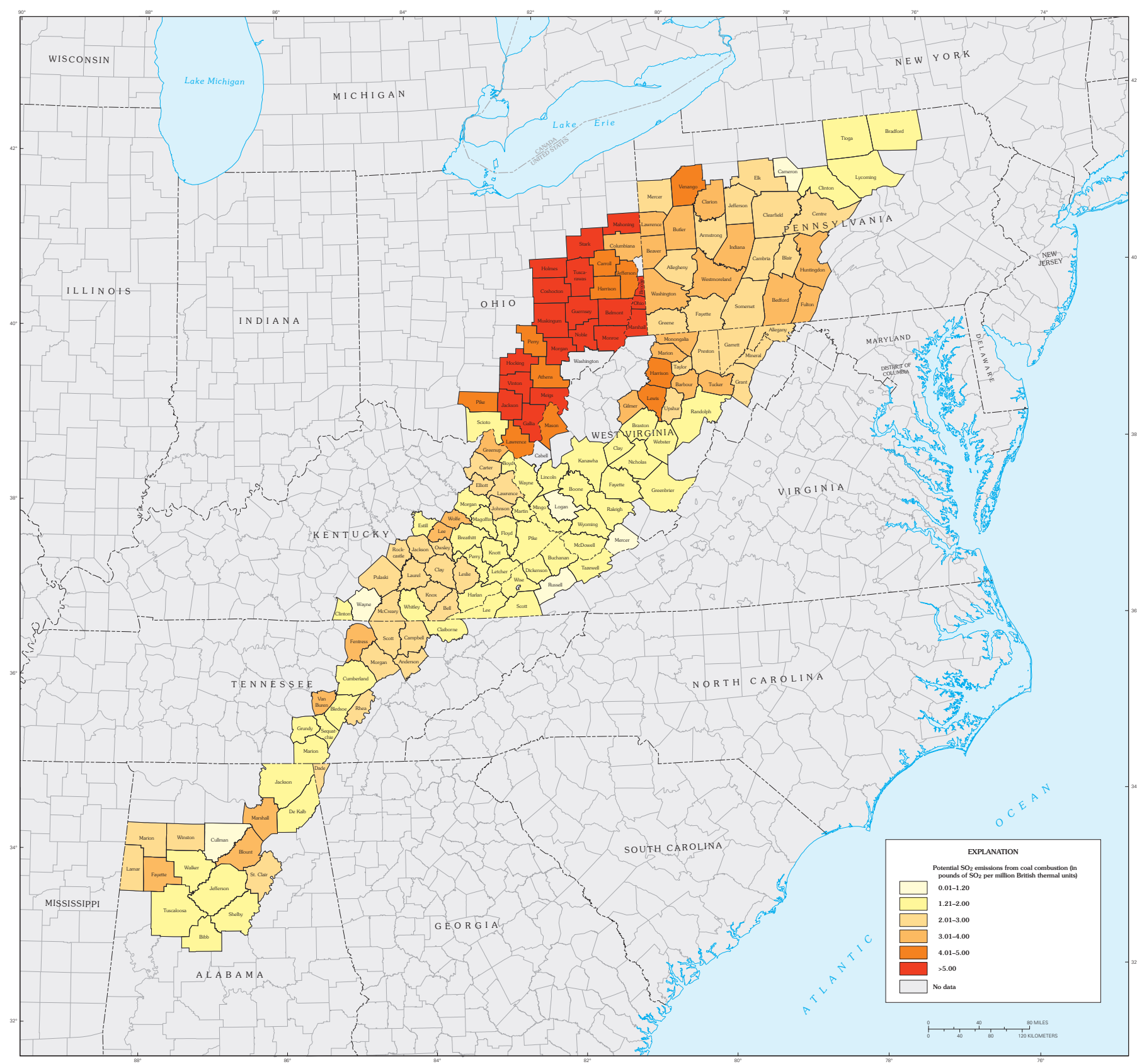

Figure 3. Potential $\mathrm{SO}_{2}$ emissions (in pounds of $\mathrm{SO}_{2}$ per million British thermal units) of bituminous coal that was mined in the Appalachian basin for electrical power generation. Data are shown by county for 1983 through 1995 and are given in table 4. 


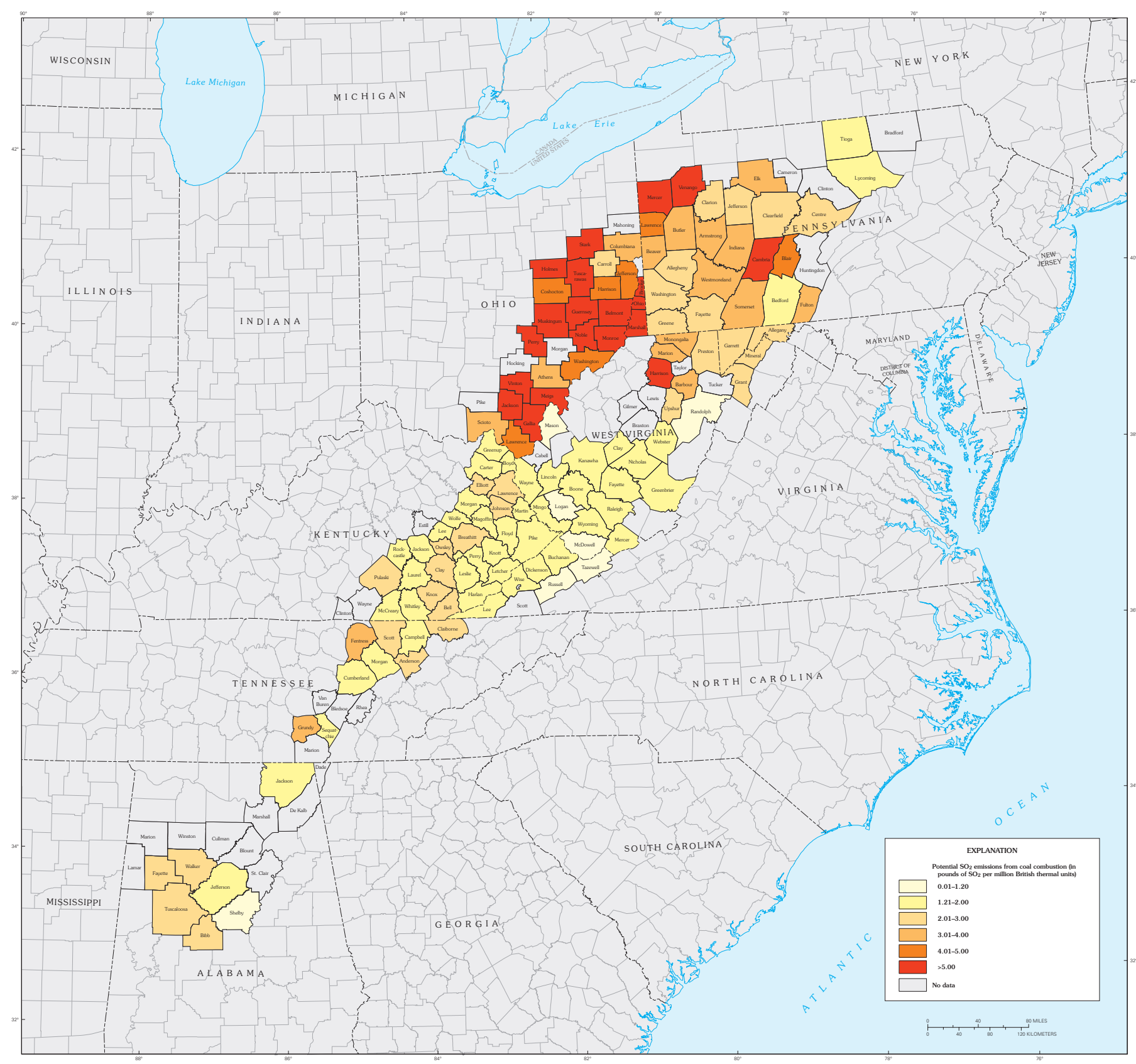

Figure 4. Potential $\mathrm{SO}_{2}$ emissions (in pounds of $\mathrm{SO}_{2}$ per million British thermal units) of bituminous coal that was mined in the Appalachian basin for electrical power generation. Data are shown by county for 1996 through 2005 and are given in table 4. 


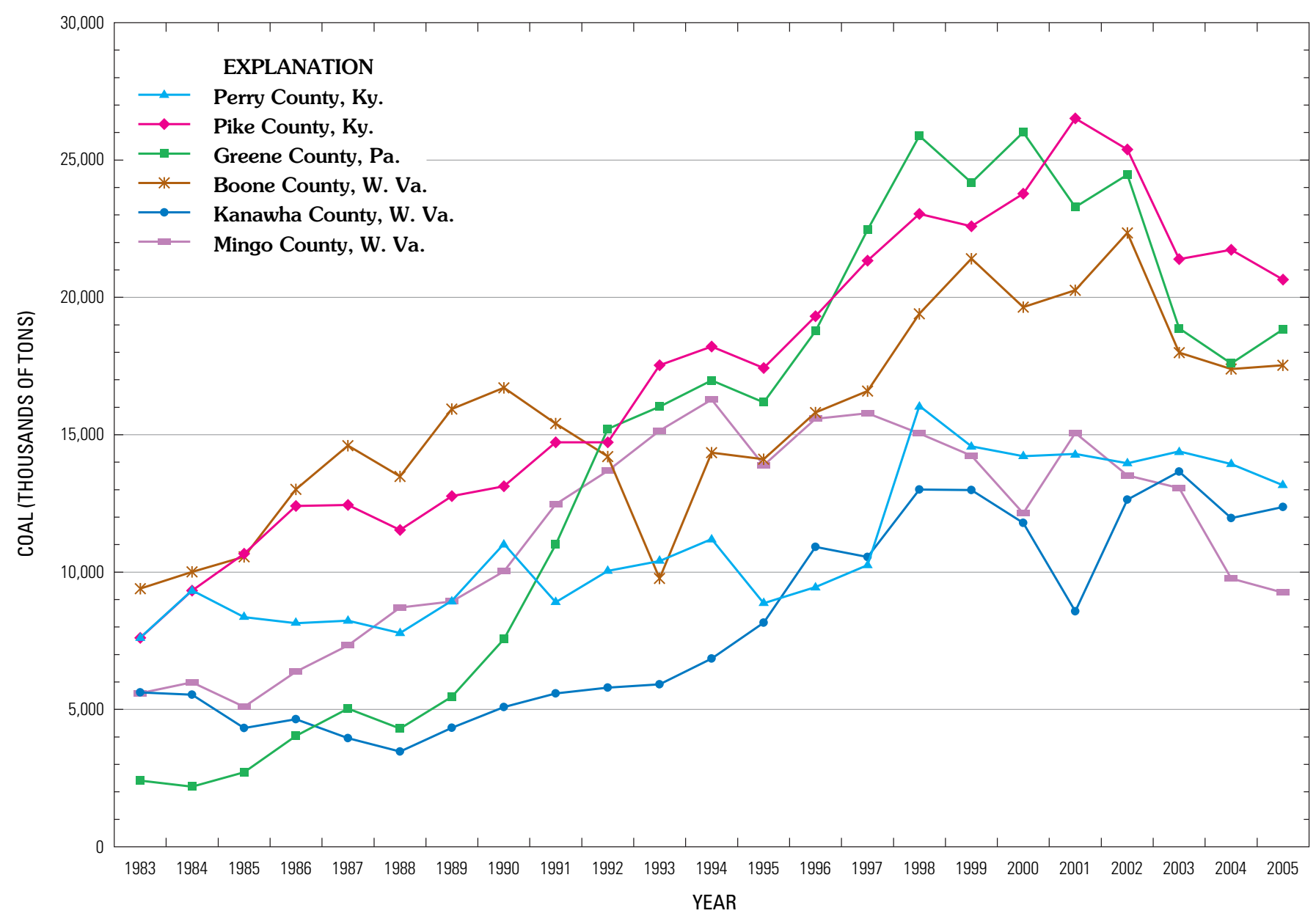

Figure 5. Amount of bituminous coal (in thousands of tons) that was mined for electrical power generation from six Appalachian basin counties that exhibited generally increasing production trends. Data are shown for 1983 through 2005 and are given in table 1. 


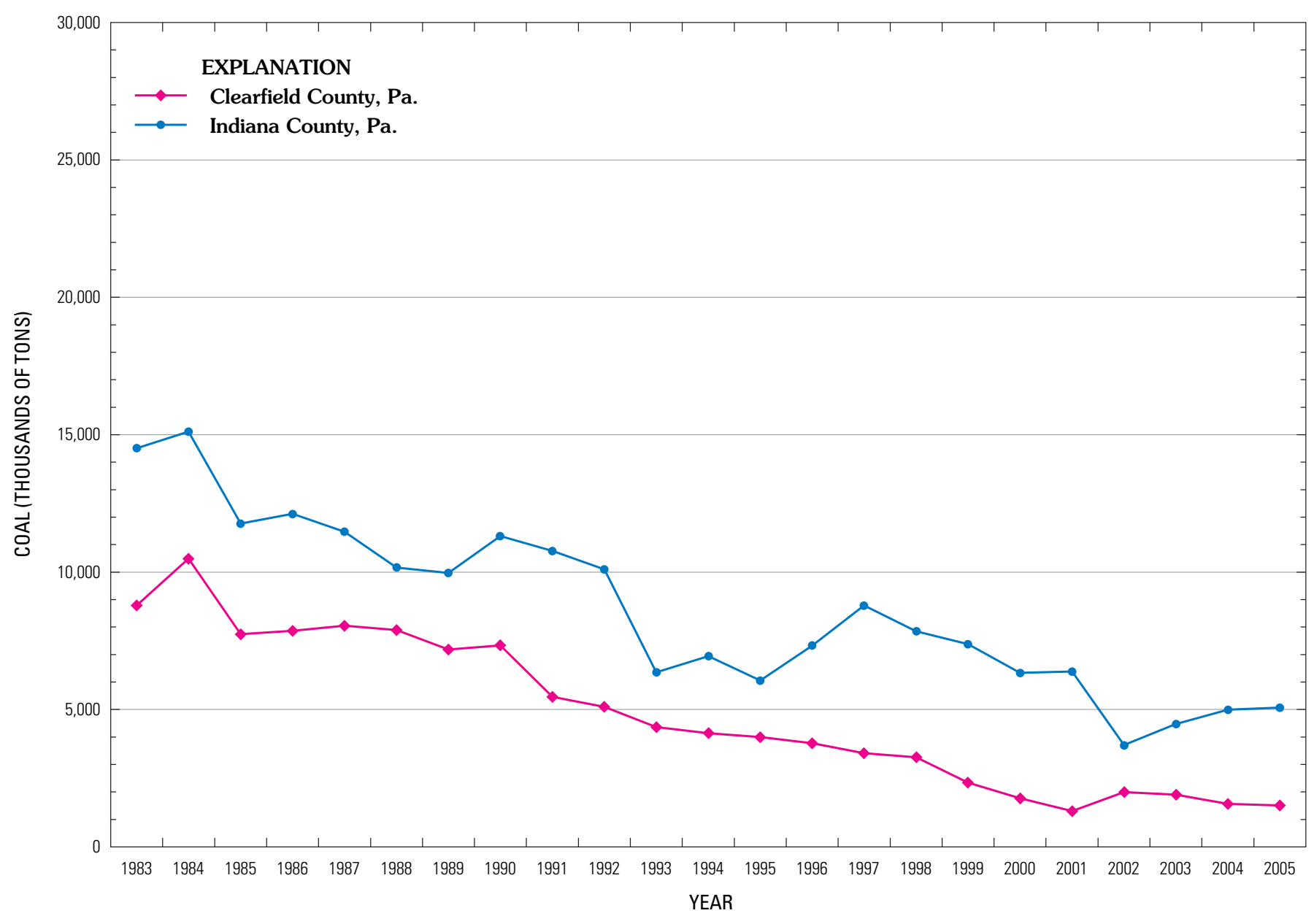

Figure 6. Amount of bituminous coal (in thousands of tons) that was mined for electrical power generation from two Appalachian basin counties that exhibited generally decreasing production trends. Data are shown for 1983 through 2005 and are given in table 1. 


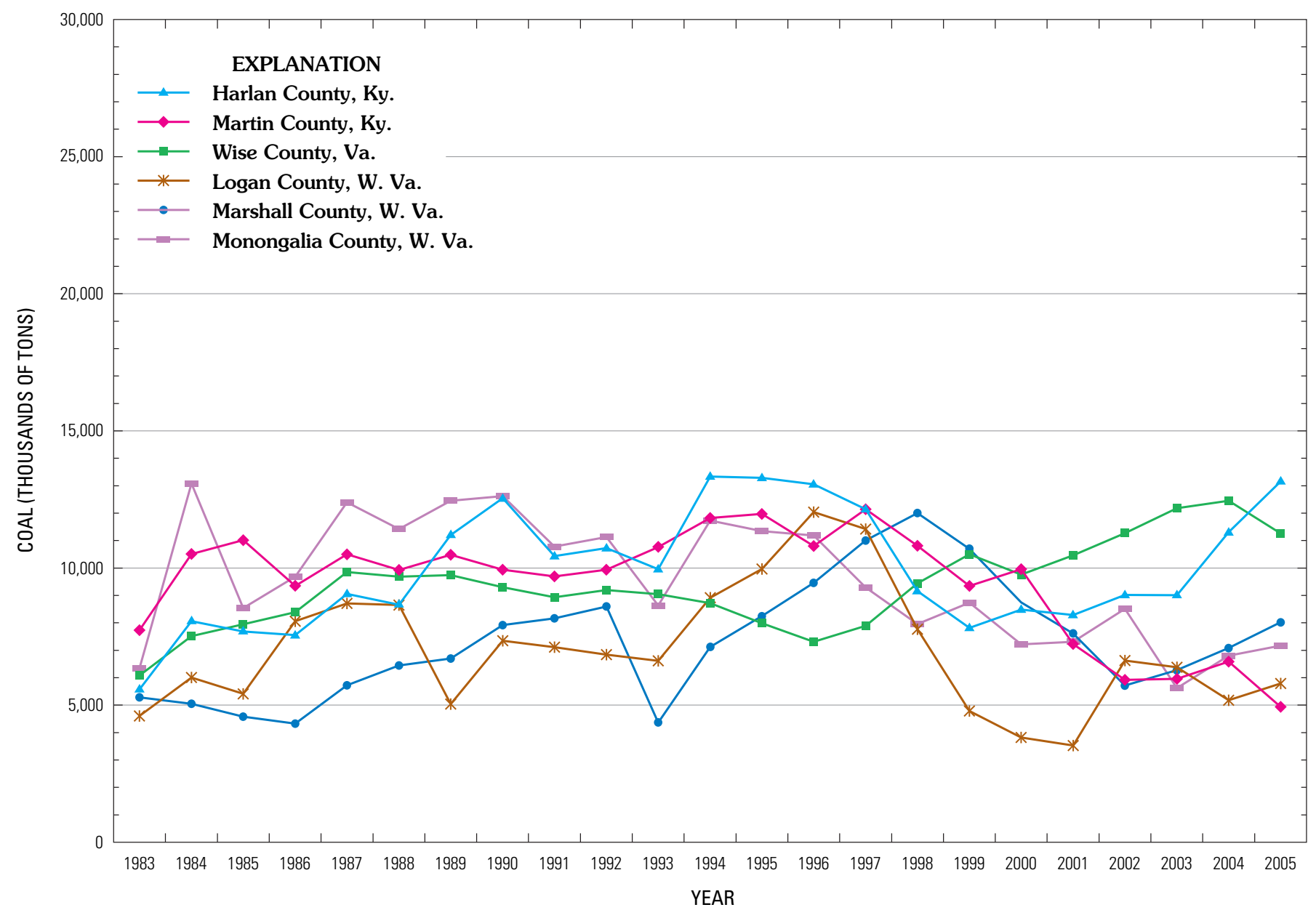

Figure 7. Amount of bituminous coal (in thousands of tons) that was mined for electrical power generation from six Appalachian basin counties that exhibited fluctuating production trends. Data are shown for 1983 through 2005 and are given in table 1. 


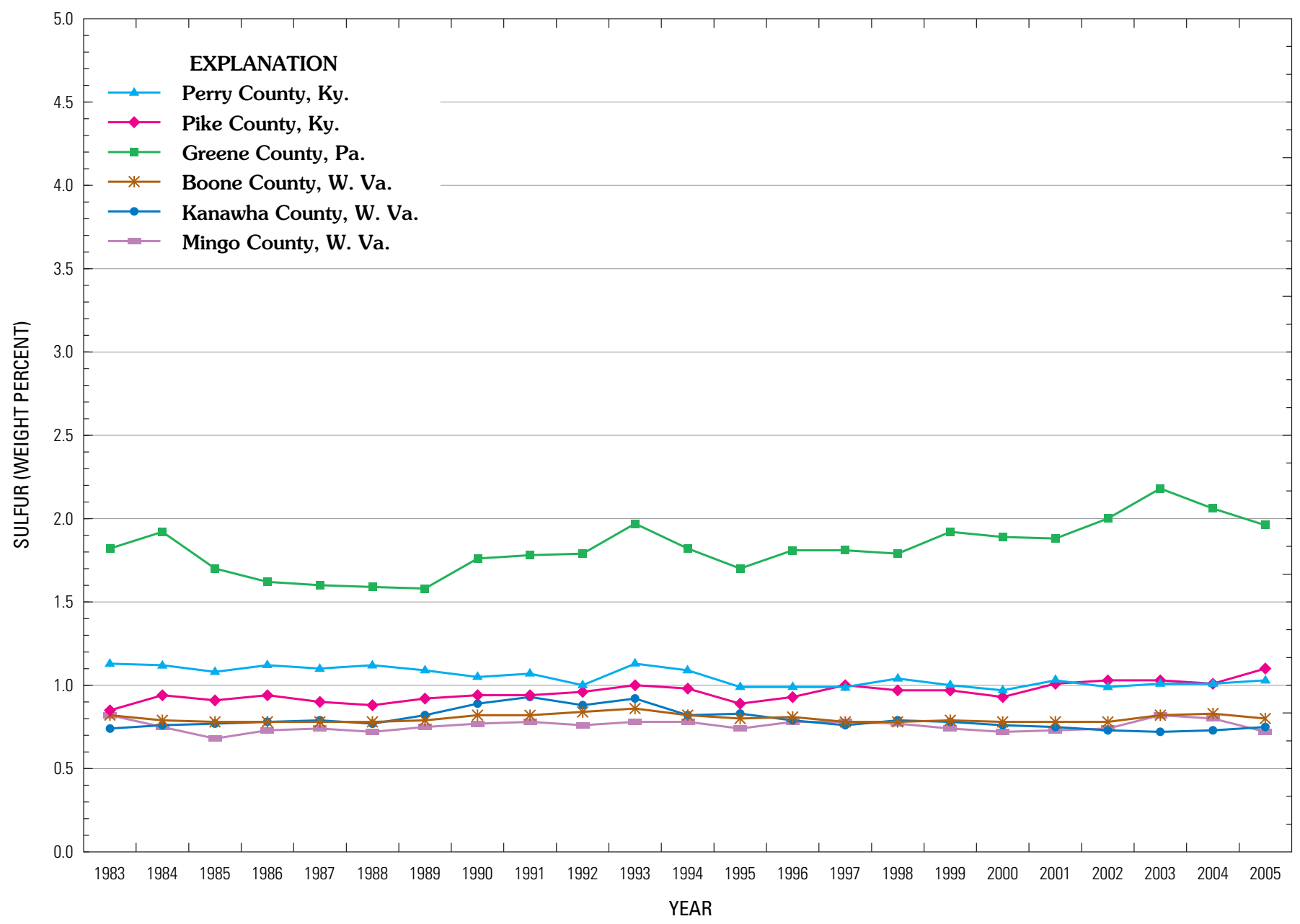

Figure 8. Sulfur content (in weight percent) of bituminous coal that was mined for electrical power generation from six Appalachian basin counties that exhibited generally increasing production trends. Data are shown for 1983 through 2005 and are given in table 3. 


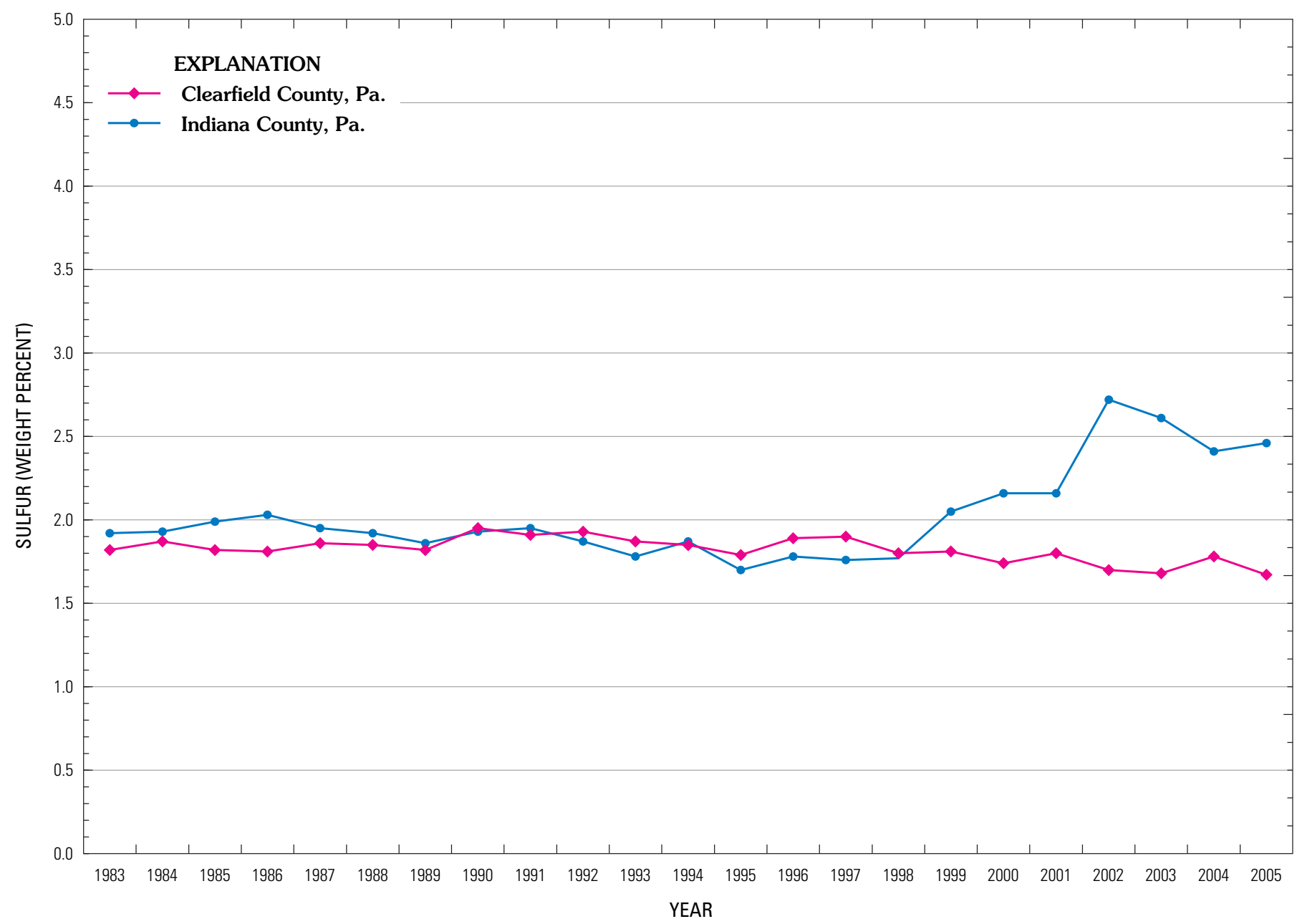

Figure 9. Sulfur content (in weight percent) of bituminous coal that was mined for electrical power generation from two Appalachian basin counties that exhibited generally decreasing production trends. Data are shown for 1983 through 2005 and are given in table 3. 


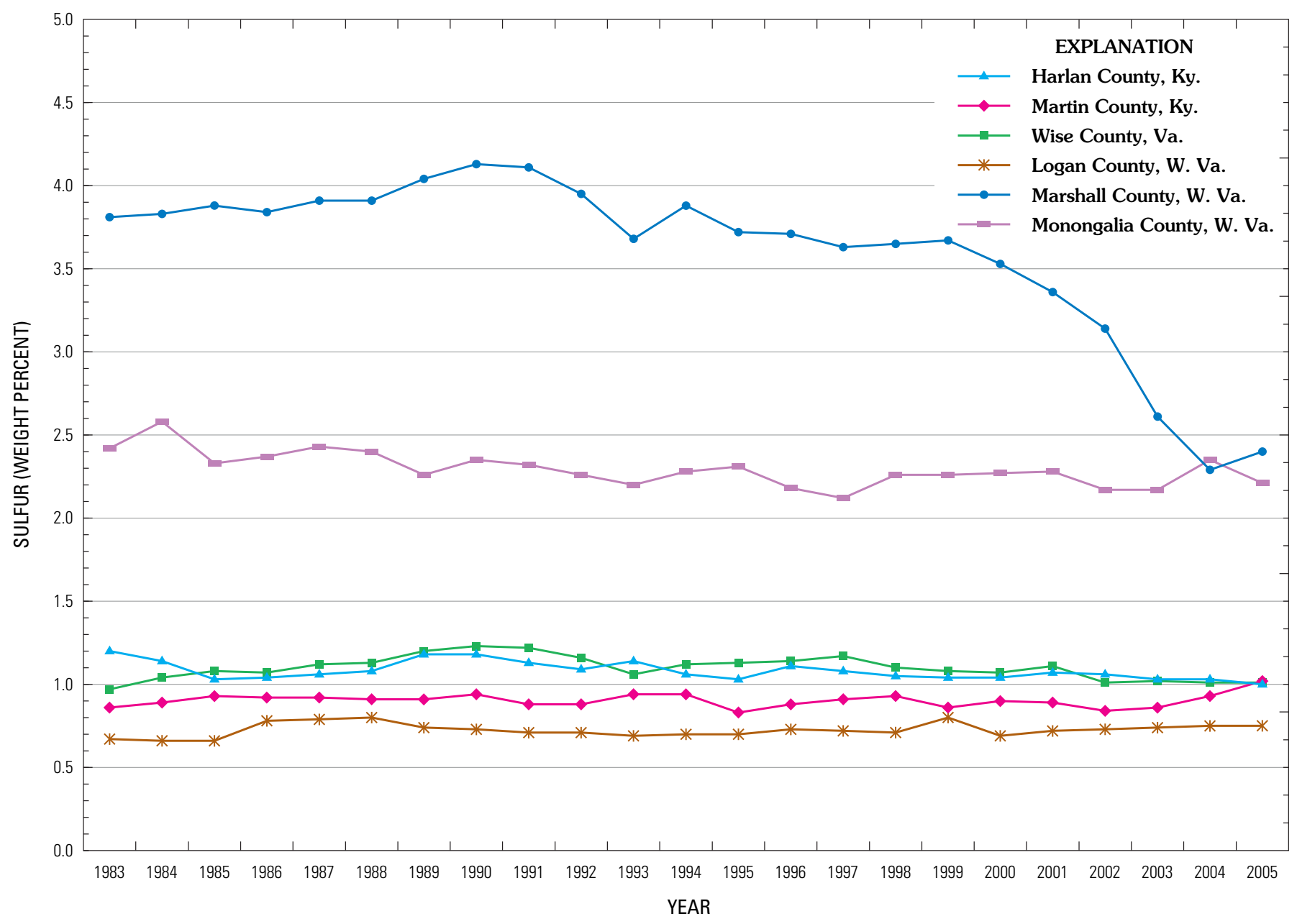

Figure 10. Sulfur content (in weight percent) of bituminous coal that was mined for electrical power generation from six Appalachian basin counties that exhibited fluctuating production trends. Data are shown for 1983 through 2005 and are given in table 3. 


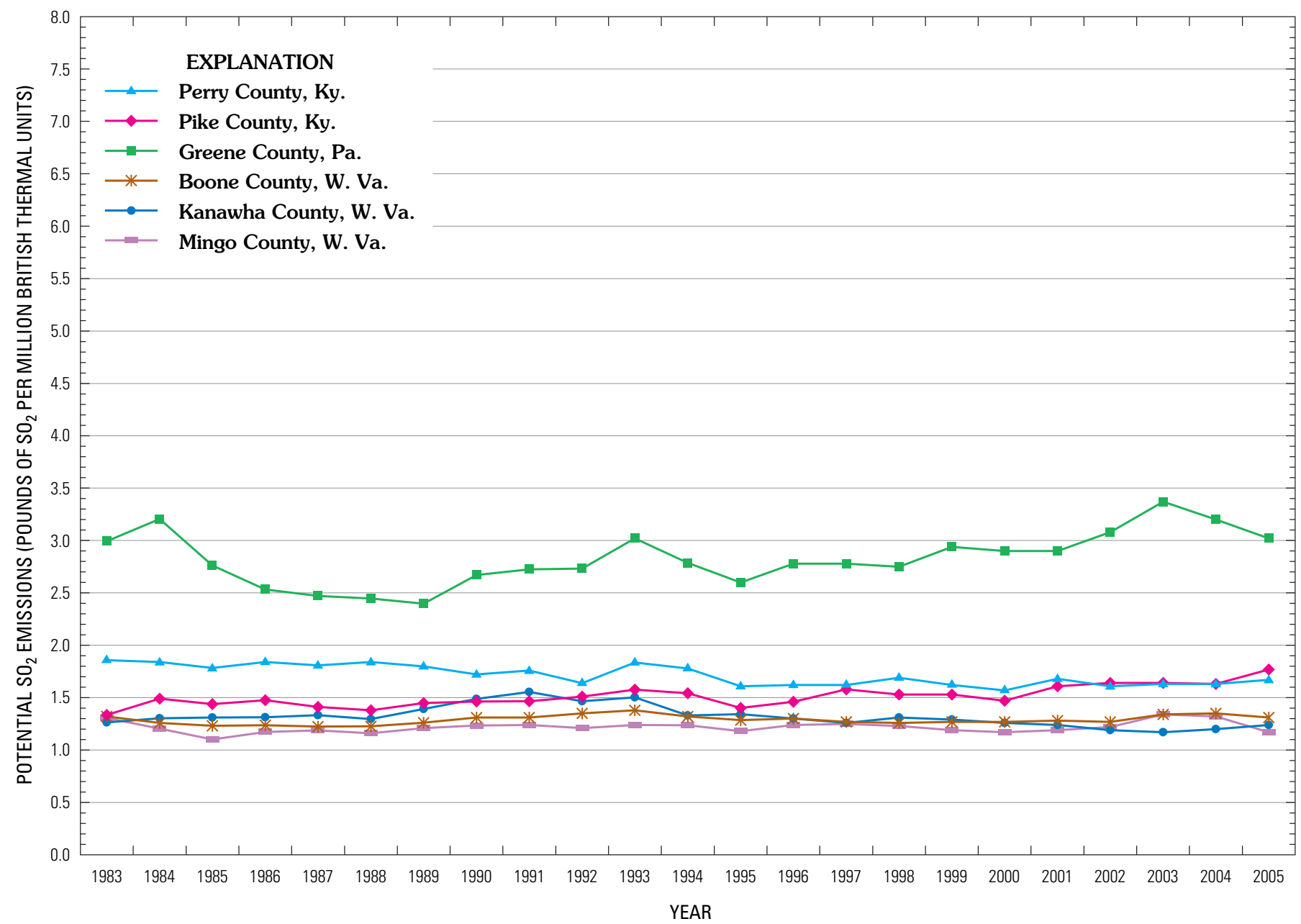

Figure 11. Potential $\mathrm{SO}_{2}$ emissions (in pounds of $\mathrm{SO}_{2}$ per million British thermal units) based on sulfur percentages of coal that was mined for electrical power generation from six Appalachian basin counties that exhibited generally increasing production trends. Data are shown for 1983 through 2005 and are given in table 4. 


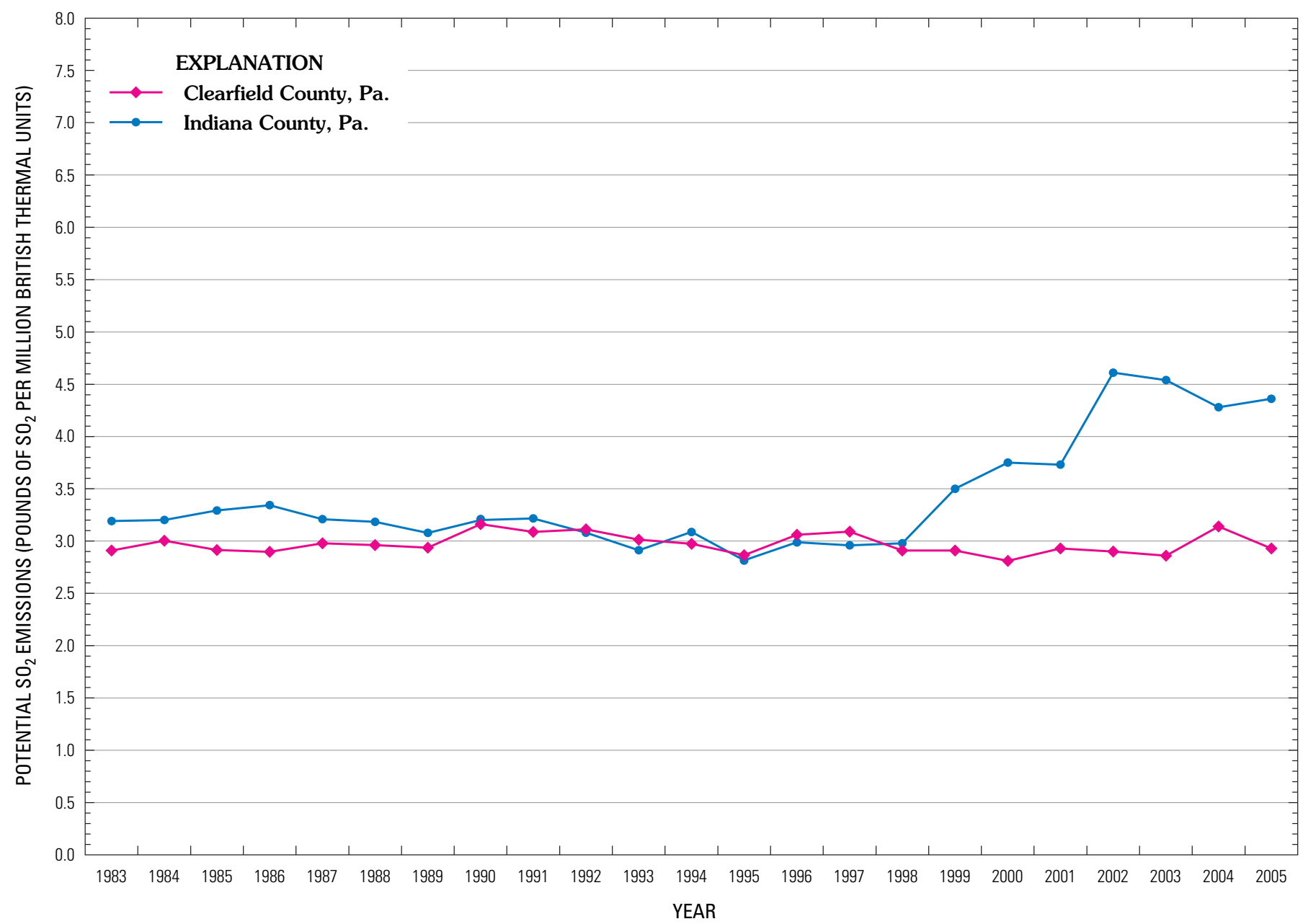

Figure 12. Potential $\mathrm{SO}_{2}$ emissions (in pounds of $\mathrm{SO}_{2}$ per million British thermal units) based on sulfur percentages of coal that was mined for electrical power generation from two Appalachian basin counties that exhibited generally decreasing production trends. Data are shown for 1983 through 2005 and are given in table 4 . 


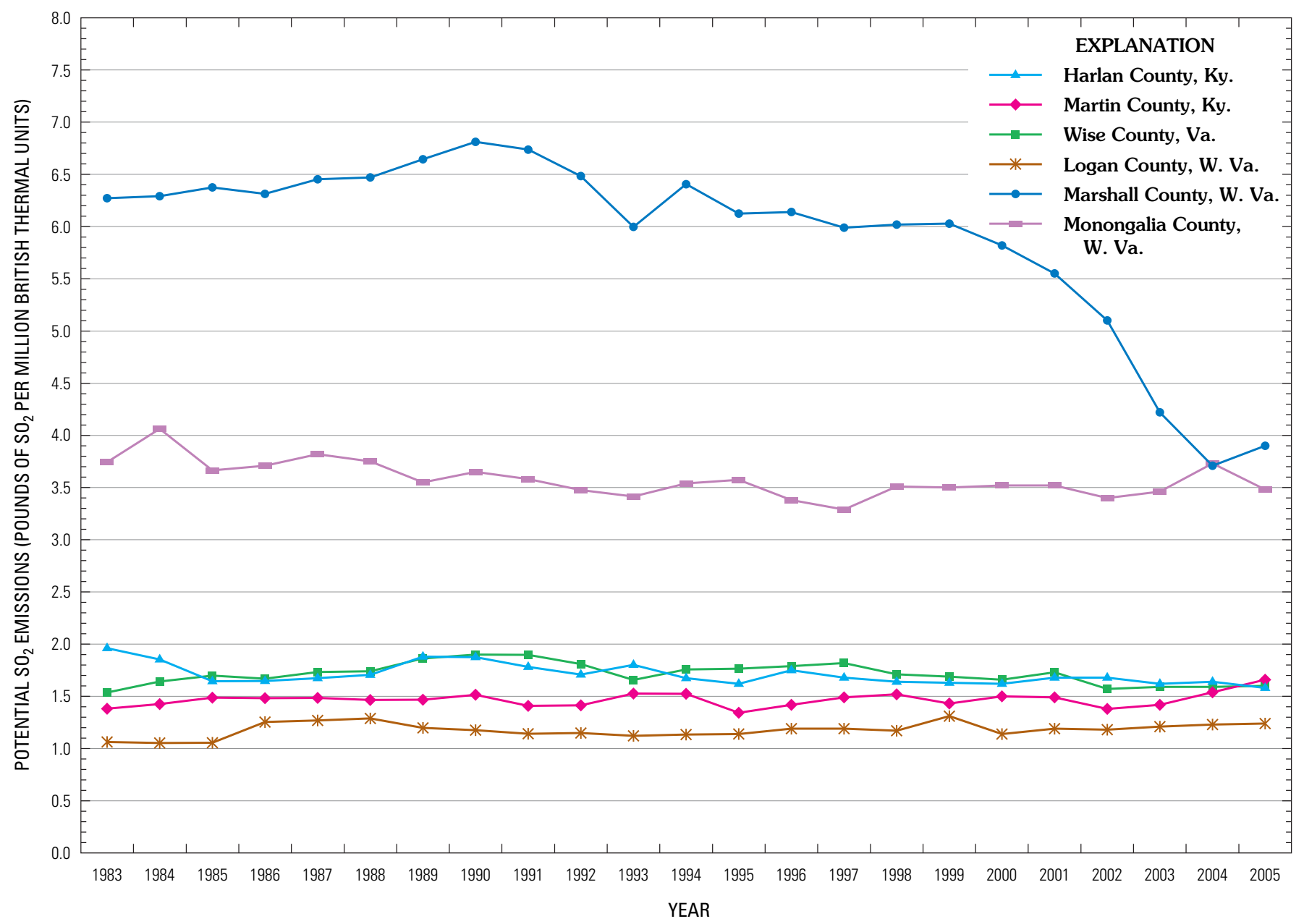

Figure 13. Potential $\mathrm{SO}_{2}$ emissions (in pounds of $\mathrm{SO}_{2}$ per million British thermal units) based on sulfur percentages of coal that was mined for electrical power generation from six Appalachian basin counties that exhibited fluctuating production trends. Data are shown for 1983 through 2005 and are given in table 4. 
Tables 1-5 
Table 1. Total weight of bituminous coal (in thousands of tons) that was mined in the Appalachian basin for electrical power generation.

[Data are shown by county. Data for 1983 through 1995 from Attanasi and Milici (1998); data for 1996 through 2005 are from the COALdat database. nd, no data]

\begin{tabular}{|c|c|c|c|c|c|c|c|c|c|c|c|c|c|c|c|}
\hline State & County & 1983 & 1984 & 1985 & 1986 & 1987 & 1988 & 1989 & 1990 & 1991 & 1992 & 1993 & 1994 & 1995 & $\begin{array}{l}\text { Total weight } \\
\text { (1983-1995) }\end{array}$ \\
\hline Ala. & Bibb & 0.00 & 0.00 & 0.00 & 0.00 & 0.00 & 0.00 & 0.00 & 0.00 & 0.00 & 0.00 & 0.00 & 0.00 & 10.00 & 10.00 \\
\hline Ala. & Blount & 0.00 & 186.00 & 582.00 & 155.00 & 17.00 & 0.00 & 0.00 & 85.00 & 9.00 & 0.00 & 0.00 & 0.00 & 0.00 & $1,034.00$ \\
\hline Ala. & Cullman & 232.00 & 162.00 & 0.00 & 12.00 & 0.00 & 0.00 & 0.00 & 0.00 & 0.00 & 0.00 & 0.00 & 0.00 & 0.00 & 406.00 \\
\hline Ala. & De Kalb & 0.00 & 0.00 & 0.00 & 24.00 & 0.00 & 0.00 & 0.00 & 0.00 & 0.00 & 0.00 & 0.00 & 0.00 & 0.00 & 24.00 \\
\hline Ala. & Fayette & $1,193.00$ & $1,127.00$ & $1,349.00$ & $1,375.00$ & $1,461.00$ & $1,127.00$ & $1,932.00$ & $2,080.00$ & $1,971.00$ & $1,878.00$ & $2,279.00$ & $1,447.00$ & $1,794.00$ & $21,013.00$ \\
\hline Ala. & Jackson & 166.00 & 125.00 & 21.00 & 13.00 & 25.00 & 0.00 & 0.00 & 0.00 & 0.00 & 0.00 & 0.00 & 100.00 & 0.00 & 450.00 \\
\hline Ala. & Jefferson & $4,096.00$ & $4,574.00$ & $5,240.00$ & $5,692.00$ & $5,643.00$ & $5,091.00$ & $3,893.00$ & $5,041.00$ & $5,328.00$ & $5,254.00$ & $5,778.00$ & $6,471.00$ & $5,562.00$ & $67,663.00$ \\
\hline Ala. & Lamar & 0.00 & 0.00 & 0.00 & 0.00 & 0.00 & 130.00 & 202.00 & 0.00 & 0.00 & 0.00 & 0.00 & 0.00 & 0.00 & 332.00 \\
\hline Ala. & Marion & 0.00 & 441.00 & 403.00 & 407.00 & 461.00 & 556.00 & 572.00 & 604.00 & 135.00 & 30.00 & 73.00 & 46.00 & 0.00 & $3,728.00$ \\
\hline Ala. & Marshall & 206.00 & 110.00 & 0.00 & 0.00 & 0.00 & 0.00 & 0.00 & 0.00 & 0.00 & 0.00 & 0.00 & 0.00 & 0.00 & 316.00 \\
\hline Ala. & St. Clair & 0.00 & 0.00 & 8.00 & 0.00 & 4.00 & 2.00 & 0.00 & 0.00 & 0.00 & 0.00 & 0.00 & 0.00 & 0.00 & 14.00 \\
\hline Ala. & Shelby & 0.00 & 0.00 & 0.00 & 0.00 & 0.00 & 0.00 & 0.00 & 0.00 & 0.00 & 0.00 & 0.00 & 0.00 & 653.00 & 653.00 \\
\hline Ala. & Tuscaloosa & 184.00 & 137.00 & 585.00 & $1,776.00$ & $1,678.00$ & $1,765.00$ & $2,522.00$ & $2,419.00$ & $2,465.00$ & $2,635.00$ & $2,025.00$ & $2,632.00$ & $3,001.00$ & $23,824.00$ \\
\hline Ala. & Walker & $8,392.00$ & $8,085.00$ & $8,176.00$ & $6,919.00$ & $5,877.00$ & $5,917.00$ & $5,833.00$ & $5,949.00$ & $7,202.00$ & $6,461.00$ & $5,973.00$ & $4,839.00$ & $4,616.00$ & $84,239.00$ \\
\hline Ala. & Winston & 0.00 & 0.00 & 183.00 & 10.00 & 10.00 & 6.00 & 25.00 & 202.00 & 94.00 & 174.00 & 268.00 & 181.00 & 0.00 & $1,153.00$ \\
\hline Ga. & Dade & 1.00 & 9.00 & 0.00 & 0.00 & 0.00 & 0.00 & 0.00 & 0.00 & 0.00 & 0.00 & 0.00 & 0.00 & 0.00 & 10.00 \\
\hline Ку. & Bell & $4,327.00$ & $5,064.00$ & $4,456.00$ & $4,637.00$ & $5,111.00$ & $5,344.00$ & $5,128.00$ & $5,060.00$ & $5,348.00$ & $4,156.00$ & $2,718.00$ & $2,908.00$ & $3,883.00$ & $58,140.00$ \\
\hline Ку. & Boyd & $1,594.00$ & $1,334.00$ & 637.00 & 756.00 & 215.00 & 173.00 & 489.00 & 230.00 & 304.00 & 512.00 & 718.00 & 939.00 & 919.00 & $8,820.00$ \\
\hline Ky. & Breathitt & $1,631.00$ & $5,455.00$ & $4,083.00$ & $4,840.00$ & $4,834.00$ & $4,057.00$ & $4,179.00$ & $4,399.00$ & $1,467.00$ & $1,664.00$ & $2,258.00$ & $3,483.00$ & $2,013.00$ & $44,363.00$ \\
\hline Ку. & Carter & 74.00 & 244.00 & 298.00 & 55.00 & 170.00 & 95.00 & 41.00 & 57.00 & 52.00 & 69.00 & 0.00 & 32.00 & 0.00 & $1,187.00$ \\
\hline Ky. & Clay & $5,795.00$ & $6,171.00$ & $3,263.00$ & $3,502.00$ & $4,188.00$ & 965.00 & 848.00 & 512.00 & $1,235.00$ & 831.00 & 205.00 & 276.00 & 170.00 & $27,961.00$ \\
\hline Ky. & Clinton & 0.00 & 0.00 & 78.00 & 0.00 & 0.00 & 0.00 & 0.00 & 0.00 & 0.00 & 0.00 & 0.00 & 0.00 & 0.00 & 78.00 \\
\hline Ky. & Elliott & 345.00 & 434.00 & 468.00 & 209.00 & 57.00 & 0.00 & 130.00 & 87.00 & 10.00 & 0.00 & 0.00 & 0.00 & 0.00 & $1,740.00$ \\
\hline Ky. & Estill & 1.00 & 0.00 & 0.00 & 42.00 & 0.00 & 0.00 & 0.00 & 0.00 & 0.00 & 0.00 & 199.00 & 43.00 & 83.00 & 368.00 \\
\hline Ку. & Floyd & $3,526.00$ & $4,473.00$ & $4,651.00$ & $4,027.00$ & $4,373.00$ & $4,353.00$ & $4,693.00$ & $5,865.00$ & $3,891.00$ & $3,590.00$ & $5,240.00$ & $5,344.00$ & $5,770.00$ & $59,796.00$ \\
\hline Ky. & Greenup & 100.00 & 434.00 & 1.00 & 21.00 & 403.00 & $1,175.00$ & 207.00 & 277.00 & 235.00 & 263.00 & 537.00 & 224.00 & 236.00 & $4,113.00$ \\
\hline Ку. & Harlan & $5,573.00$ & $8,059.00$ & $7,683.00$ & $7,552.00$ & $9,060.00$ & $8,663.00$ & $11,205.00$ & $12,550.00$ & $10,435.00$ & $10,730.00$ & $9,958.00$ & $13,337.00$ & $13,285.00$ & $128,090.00$ \\
\hline Ky. & Jackson & 44.00 & 159.00 & 29.00 & 91.00 & 0.00 & 5.00 & 1.00 & 27.00 & 1.00 & 8.00 & 0.00 & 84.00 & 108.00 & 557.00 \\
\hline Ky. & Johnson & $1,951.00$ & $2,095.00$ & 727.00 & 893.00 & $1,309.00$ & 824.00 & 802.00 & $1,050.00$ & 845.00 & 905.00 & $1,051.00$ & 738.00 & 679.00 & $13,869.00$ \\
\hline Ky. & Knott & 316.00 & $1,096.00$ & $1,065.00$ & $1,080.00$ & 743.00 & 463.00 & 915.00 & $1,615.00$ & $2,844.00$ & $3,925.00$ & $4,786.00$ & $5,277.00$ & $4,488.00$ & $28,613.00$ \\
\hline
\end{tabular}


Table 1. Total weight of bituminous coal (in thousands of tons) that was mined in the Appalachian basin for electrical power generation.-Continued

[Data are shown by county. Data for 1983 through 1995 from Attanasi and Milici (1998); data for 1996 through 2005 are from the COALdat database. nd, no data]

\begin{tabular}{|c|c|c|c|c|c|c|c|c|c|c|c|c|}
\hline State & County & 1996 & 1997 & 1998 & 1999 & 2000 & 2001 & 2002 & 2003 & 2004 & 2005 & $\begin{array}{l}\text { Total weight } \\
\text { (1996-2005) }\end{array}$ \\
\hline Ala. & Bibb & 120.25 & 126.62 & 92.74 & 0.00 & 0.00 & 0.00 & 0.00 & 0.00 & 34.02 & 252.35 & 625.98 \\
\hline Ala. & Blount & nd & nd & nd & nd & nd & nd & nd & nd & nd & nd & 0.00 \\
\hline Ala. & Cullman & nd & nd & nd & nd & nd & nd & nd & nd & nd & nd & 0.00 \\
\hline Ala. & De Kalb & nd & nd & nd & nd & $\mathrm{nd}$ & nd & nd & nd & nd & nd & 0.00 \\
\hline Ala. & Fayette & 69.61 & 0.00 & 0.00 & 0.00 & 0.00 & 598.43 & 203.28 & 0.00 & 0.00 & 0.00 & 871.32 \\
\hline Ala. & Jackson & 0.00 & 0.00 & 0.00 & 0.00 & 2.51 & 0.00 & 0.00 & 58.78 & 81.47 & 117.20 & 259.96 \\
\hline Ala. & Jefferson & $8,112.49$ & $6,750.74$ & $6,610.95$ & $5,339.09$ & $4,599.87$ & $4,132.65$ & $4,703.23$ & $4,825.86$ & $4,534.94$ & $3,162.36$ & $52,772.18$ \\
\hline Ala. & Lamar & nd & nd & nd & nd & nd & nd & $\mathrm{nd}$ & nd & nd & nd & 0.00 \\
\hline Ala. & Marion & nd & nd & nd & nd & nd & nd & nd & nd & nd & nd & 0.00 \\
\hline Ala. & Marshall & nd & nd & nd & nd & nd & nd & nd & nd & nd & nd & 0.00 \\
\hline Ala. & St. Clair & nd & nd & nd & nd & nd & nd & nd & nd & nd & nd & 0.00 \\
\hline Ala. & Shelby & 400.02 & 689.45 & 441.80 & 0.00 & 12.67 & 63.27 & 0.00 & 0.00 & 0.00 & 0.00 & $1,607.21$ \\
\hline Ala. & Tuscaloosa & $4,372.08$ & $4,154.38$ & $5,681.69$ & $5,220.77$ & $4,539.68$ & $2,288.80$ & 789.19 & $2,405.19$ & $1,626.68$ & $4,513.99$ & $35,592.45$ \\
\hline Ala. & Walker & $3,747.40$ & $4,311.24$ & $3,849.30$ & $2,706.75$ & $1,863.30$ & $5,219.12$ & $5,480.38$ & $4,653.24$ & $5,266.73$ & $2,165.56$ & $39,263.02$ \\
\hline Ala. & Winston & nd & nd & nd & nd & nd & nd & nd & nd & nd & nd & 0.00 \\
\hline Ga. & Dade & nd & nd & nd & $\mathrm{nd}$ & nd & nd & nd & $\mathrm{nd}$ & nd & nd & 0.00 \\
\hline Ky. & Bell & $4,122.35$ & $3,605.60$ & $3,386.82$ & $2,814.66$ & $1,579.84$ & 690.35 & $1,425.22$ & $1,251.70$ & 978.97 & $1,557.19$ & $21,412.70$ \\
\hline Ky. & Boyd & 303.05 & 397.72 & 387.87 & 412.50 & 821.33 & $1,256.99$ & $1,102.14$ & 740.23 & 857.63 & 422.25 & $6,701.71$ \\
\hline Ky. & Breathitt & $1,042.61$ & $1,390.26$ & 737.13 & 496.33 & 229.30 & 271.30 & 347.69 & 524.47 & 488.26 & 223.69 & $5,751.04$ \\
\hline Ky. & Carter & 0.00 & 0.00 & 79.00 & 0.00 & 0.00 & 0.00 & 0.00 & 0.00 & 0.00 & 0.00 & 79.00 \\
\hline Ky. & Clay & 628.40 & 508.00 & 411.60 & 490.80 & 173.40 & 403.75 & 266.64 & 194.32 & 362.00 & 515.01 & $3,953.92$ \\
\hline Ky. & Clinton & nd & nd & nd & nd & nd & nd & nd & nd & nd & nd & 0.00 \\
\hline Ку. & Elliott & nd & nd & nd & nd & nd & nd & nd & nd & nd & nd & 0.00 \\
\hline Ky. & Estill & 9.50 & 10.00 & 0.00 & 9.14 & 220.30 & 295.30 & 53.00 & 52.00 & 20.00 & 0.00 & 669.24 \\
\hline Ky. & Floyd & $4,650.69$ & $4,013.14$ & $4,879.03$ & $3,024.67$ & $2,323.98$ & 839.26 & $1,371.72$ & 958.84 & $1,724.11$ & $1,990.17$ & $25,775.61$ \\
\hline Ky. & Greenup & 201.00 & 266.00 & 264.00 & 172.00 & 173.00 & 8.00 & 0.00 & 0.00 & 0.00 & 0.00 & $1,084.00$ \\
\hline Ky. & Harlan & $13,055.86$ & $12,143.29$ & $9,159.63$ & $7,820.85$ & $8,483.03$ & $8,285.30$ & $9,021.87$ & $9,004.16$ & $11,302.96$ & $13,158.26$ & $101,435.21$ \\
\hline Ку. & Jackson & 0.00 & 0.00 & 0.00 & 0.00 & 0.00 & 0.00 & 8.75 & 0.00 & 0.00 & 0.00 & 8.75 \\
\hline Ky. & Johnson & $1,386.85$ & $1,895.80$ & $1,536.60$ & $1,429.65$ & $1,085.70$ & $1,708.80$ & $1,225.22$ & $1,202.67$ & 305.38 & 137.60 & $11,914.27$ \\
\hline Ky. & Knott & $5,778.05$ & $7,025.75$ & $6,588.82$ & $6,998.43$ & $6,878.00$ & $7,969.58$ & $8,504.12$ & $5,696.82$ & $7,419.92$ & $7,620.92$ & $70,480.41$ \\
\hline
\end{tabular}


Table 1. Total weight of bituminous coal (in thousands of tons) that was mined in the Appalachian basin for electrical power generation.-Continued [Data are shown by county. Data for 1983 through 1995 from Attanasi and Milici (1998); data for 1996 through 2005 are from the COALdat database. nd, no data]

\begin{tabular}{|c|c|c|c|c|c|c|c|c|c|c|c|c|c|c|c|}
\hline State & County & 1983 & 1984 & 1985 & 1986 & 1987 & 1988 & 1989 & 1990 & 1991 & 1992 & 1993 & 1994 & 1995 & $\begin{array}{l}\text { Total weight } \\
\text { (1983-1995) }\end{array}$ \\
\hline Ky. & Knox & 289.00 & 542.00 & 693.00 & 678.00 & 499.00 & 296.00 & 271.00 & 9.00 & 15.00 & 14.00 & 35.00 & 17.00 & 74.00 & $3,432.00$ \\
\hline Ky. & Laurel & 472.00 & $1,350.00$ & 869.00 & 985.00 & 907.00 & 999.00 & 152.00 & 436.00 & 7.00 & 12.00 & 22.00 & 0.00 & 1.00 & $6,212.00$ \\
\hline Ку. & Lawrence & 118.00 & 328.00 & 175.00 & 246.00 & 515.00 & 408.00 & 417.00 & 225.00 & 56.00 & 125.00 & 190.00 & 349.00 & 92.00 & $3,244.00$ \\
\hline Ky. & Lee & 0.00 & 0.00 & 278.00 & 165.00 & 51.00 & 17.00 & 48.00 & 28.00 & 0.00 & 0.00 & 7.00 & 0.00 & 5.00 & 599.00 \\
\hline Ky. & Leslie & $2,751.00$ & $2,966.00$ & $3,459.00$ & $2,818.00$ & $2,964.00$ & $4,185.00$ & $4,327.00$ & $4,763.00$ & $4,441.00$ & $5,671.00$ & $7,094.00$ & $6,962.00$ & $7,517.00$ & $59,918.00$ \\
\hline Ку. & Letcher & $1,701.00$ & $3,254.00$ & $3,270.00$ & $4,389.00$ & $5,357.00$ & $7,004.00$ & $7,284.00$ & $7,735.00$ & $5,580.00$ & $5,385.00$ & $7,635.00$ & $6,748.00$ & $7,267.00$ & $72,609.00$ \\
\hline Ку. & McCreary & 786.00 & 817.00 & $1,725.00$ & $1,518.00$ & $1,378.00$ & 533.00 & 0.00 & 0.00 & 0.00 & 25.00 & 186.00 & 24.00 & 59.00 & $7,051.00$ \\
\hline Ку. & Magoffin & $1,238.00$ & $1,662.00$ & $2,437.00$ & $2,034.00$ & $1,407.00$ & $1,373.00$ & $1,890.00$ & $1,907.00$ & $1,680.00$ & $1,342.00$ & 810.00 & 955.00 & 911.00 & $19,646.00$ \\
\hline Ку. & Martin & $7,731.00$ & $10,507.00$ & $11,013.00$ & $9,344.00$ & $10,504.00$ & $9,928.00$ & $10,480.00$ & $9,939.00$ & $9,695.00$ & $9,933.00$ & $10,759.00$ & $11,822.00$ & $11,970.00$ & $133,625.00$ \\
\hline Ky. & Morgan & 31.00 & 17.00 & 3.00 & 90.00 & 149.00 & 1.00 & 6.00 & 24.00 & 0.00 & 0.00 & 0.00 & 19.00 & 0.00 & 340.00 \\
\hline Ку. & Owsley & 31.00 & 122.00 & 82.00 & 248.00 & 65.00 & 205.00 & 157.00 & 28.00 & 18.00 & 8.00 & 17.00 & 10.00 & 0.00 & 991.00 \\
\hline Ку. & Perry & $7,613.00$ & $9,331.00$ & $8,356.00$ & $8,135.00$ & $8,232.00$ & $7,777.00$ & $8,961.00$ & $11,025.00$ & $8,902.00$ & $10,044.00$ & $10,399.00$ & $11,202.00$ & $8,869.00$ & $118,846.00$ \\
\hline Ky. & Pike & $7,606.00$ & $9,326.00$ & $10,669.00$ & $12,410.00$ & $12,445.00$ & $11,533.00$ & $12,768.00$ & $13,121.00$ & $14,727.00$ & $14,723.00$ & $17,532.00$ & $18,208.00$ & $17,430.00$ & $172,498.00$ \\
\hline Ку. & Pulaski & 553.00 & 721.00 & 484.00 & 401.00 & 514.00 & 459.00 & 432.00 & 419.00 & 388.00 & 394.00 & 365.00 & 417.00 & 318.00 & $5,865.00$ \\
\hline Ку. & Rockcastle & 28.00 & 0.00 & 1.00 & 0.00 & 0.00 & 0.00 & 0.00 & 0.00 & 0.00 & 0.00 & 2.00 & 0.00 & 0.00 & 31.00 \\
\hline Kу. & Wayne & 0.00 & 0.00 & 0.00 & 77.00 & 0.00 & 0.00 & 0.00 & 0.00 & 0.00 & 0.00 & 0.00 & 0.00 & 0.00 & 77.00 \\
\hline Ку. & Whitley & $1,514.00$ & $2,055.00$ & $2,044.00$ & $1,948.00$ & $2,994.00$ & $3,063.00$ & $3,197.00$ & $2,161.00$ & $1,811.00$ & $1,833.00$ & $1,948.00$ & $1,561.00$ & $1,152.00$ & $27,281.00$ \\
\hline Ку. & Wolfe & 2.00 & 99.00 & 235.00 & 481.00 & 399.00 & 53.00 & 28.00 & 0.00 & 59.00 & 169.00 & 213.00 & 135.00 & 14.00 & $1,887.00$ \\
\hline Md. & Allegany & 290.00 & 478.00 & 118.00 & 453.00 & 706.00 & 513.00 & 534.00 & 573.00 & 394.00 & 321.00 & 174.00 & 162.00 & 97.00 & $4,813.00$ \\
\hline Md. & Garrett & $1,418.00$ & $1,708.00$ & $1,042.00$ & $1,581.00$ & $2,111.00$ & $1,959.00$ & $1,830.00$ & $2,430.00$ & $3,118.00$ & $2,901.00$ & $2,809.00$ & $2,814.00$ & $3,125.00$ & $28,846.00$ \\
\hline Ohio & Athens & 6.00 & 0.00 & 0.00 & 0.00 & 128.00 & 103.00 & 96.00 & 74.00 & 47.00 & 0.00 & 0.00 & 0.00 & 0.00 & 454.00 \\
\hline Ohio & Belmont & $8,682.00$ & $8,470.00$ & $6,462.00$ & $6,427.00$ & $6,468.00$ & $6,175.00$ & $6,583.00$ & $5,954.00$ & $4,882.00$ & $4,877.00$ & $5,474.00$ & $6,089.00$ & $4,730.00$ & $81,273.00$ \\
\hline Ohio & Carroll & 103.00 & 167.00 & 247.00 & 238.00 & 236.00 & 153.00 & 130.00 & 65.00 & 41.00 & 505.00 & 574.00 & 444.00 & 185.00 & $3,088.00$ \\
\hline Ohio & Columbiana & 395.00 & 563.00 & 754.00 & 938.00 & 867.00 & $1,071.00$ & $1,008.00$ & $1,043.00$ & 562.00 & 429.00 & 661.00 & 905.00 & 780.00 & $9,976.00$ \\
\hline Ohio & Coshocton & $1,354.00$ & $1,178.00$ & $1,543.00$ & $1,923.00$ & $1,867.00$ & $1,785.00$ & $2,123.00$ & $2,036.00$ & $1,763.00$ & $1,894.00$ & $1,884.00$ & $1,802.00$ & $1,625.00$ & $22,777.00$ \\
\hline Ohio & Gallia & 207.00 & 399.00 & 263.00 & 236.00 & 72.00 & 0.00 & 0.00 & 0.00 & 0.00 & 0.00 & 207.00 & 336.00 & 307.00 & $2,027.00$ \\
\hline Ohio & Guernsey & 0.00 & 10.00 & 0.00 & 0.00 & 0.00 & 0.00 & 138.00 & 121.00 & 4.00 & 66.00 & 58.00 & 70.00 & 35.00 & 502.00 \\
\hline Ohio & Harrison & $2,898.00$ & $3,224.00$ & $2,579.00$ & $2,691.00$ & $2,536.00$ & $1,668.00$ & $2,197.00$ & $2,132.00$ & $3,475.00$ & $3,853.00$ & $3,512.00$ & $3,156.00$ & $2,359.00$ & $36,280.00$ \\
\hline Ohio & Hocking & 377.00 & $1,003.00$ & 762.00 & 894.00 & 902.00 & 629.00 & 804.00 & 904.00 & 561.00 & 463.00 & 217.00 & 6.00 & 0.00 & $7,522.00$ \\
\hline Ohio & Holmes & 0.00 & 0.00 & 0.00 & 0.00 & 0.00 & 23.00 & 293.00 & 313.00 & 336.00 & 332.00 & 350.00 & 399.00 & 222.00 & $2,268.00$ \\
\hline
\end{tabular}


Table 1. Total weight of bituminous coal (in thousands of tons) that was mined in the Appalachian basin for electrical power generation.-Continued [Data are shown by county. Data for 1983 through 1995 from Attanasi and Milici (1998); data for 1996 through 2005 are from the COALdat database. nd, no data]

\begin{tabular}{|c|c|c|c|c|c|c|c|c|c|c|c|c|}
\hline State & County & 1996 & 1997 & 1998 & 1999 & 2000 & 2001 & 2002 & 2003 & 2004 & 2005 & $\begin{array}{l}\text { Total weight } \\
\text { (1996-2005) }\end{array}$ \\
\hline Ky. & Knox & 0.00 & 0.00 & 21.80 & 0.00 & 0.00 & 0.00 & 25.43 & 38.24 & 118.40 & 24.72 & 228.59 \\
\hline Ky. & Laurel & 1.00 & 0.00 & 5.93 & 3.60 & 6.00 & 1.00 & 0.00 & 0.60 & 21.22 & 0.00 & 39.35 \\
\hline Ку. & Lawrence & 84.33 & 51.50 & 409.90 & 929.47 & $1,870.75$ & $2,869.19$ & $2,359.44$ & $4,152.60$ & $4,453.25$ & $3,384.21$ & $20,564.64$ \\
\hline Ky. & Lee & 0.00 & 0.00 & 0.00 & 0.00 & 0.00 & 0.00 & 5.09 & 0.00 & 0.00 & 0.00 & 5.09 \\
\hline Ky. & Leslie & $4,737.13$ & $5,202.07$ & $2,923.65$ & $3,436.06$ & $2,130.07$ & $2,570.72$ & 773.86 & 97.40 & 422.19 & 604.93 & $22,898.08$ \\
\hline Ку. & Letcher & $6,067.85$ & $5,703.18$ & $7,000.65$ & $5,164.35$ & $2,936.06$ & $4,940.28$ & $4,548.43$ & $8,098.47$ & $7,663.69$ & $6,570.17$ & $58,693.13$ \\
\hline Ky. & McCreary & 48.94 & 0.00 & 0.00 & 0.00 & 0.00 & 0.00 & 0.00 & 0.00 & 0.00 & 0.00 & 48.94 \\
\hline Ку. & Magoffin & 542.51 & 427.65 & 936.20 & 252.29 & 215.70 & 229.60 & 335.10 & 234.60 & 337.49 & 377.20 & $3,888.34$ \\
\hline Ky. & Martin & $10,799.70$ & $12,145.33$ & $10,808.79$ & $9,345.58$ & $9,957.19$ & $7,222.13$ & $5,919.34$ & $5,956.65$ & $6,588.29$ & $4,933.41$ & $83,676.41$ \\
\hline Ky. & Morgan & 0.00 & 0.00 & 12.60 & 12.60 & 36.80 & 94.20 & 0.00 & 0.00 & 0.00 & 0.00 & 156.20 \\
\hline Ky. & Owsley & 0.00 & 0.00 & 10.40 & 14.00 & 0.00 & 0.00 & 6.82 & 31.85 & 19.62 & 14.90 & 97.59 \\
\hline Ky. & Perry & $9,458.24$ & $10,250.25$ & $16,045.58$ & $14,578.61$ & $14,222.48$ & $14,302.38$ & $13,961.96$ & $14,395.93$ & $13,932.90$ & $13,161.35$ & $134,309.68$ \\
\hline Ky. & Pike & $19,316.84$ & $21,340.31$ & $23,038.98$ & $22,591.93$ & $23,772.24$ & $26,514.41$ & $25,384.20$ & $21,391.08$ & $21,732.54$ & $20,645.98$ & $225,728.51$ \\
\hline Ky. & Pulaski & 0.00 & 0.00 & 9.00 & 226.16 & 593.71 & 340.00 & 513.00 & 656.32 & 166.80 & 0.00 & $2,504.99$ \\
\hline Ky. & Rockcastle & 0.00 & 0.00 & 0.00 & 0.00 & 0.00 & 0.00 & 0.00 & 0.00 & 0.00 & 66.00 & 66.00 \\
\hline Ky. & Wayne & nd & nd & nd & nd & nd & nd & nd & nd & nd & nd & 0.00 \\
\hline Ky. & Whitley & 797.75 & 484.37 & 333.74 & 234.63 & 48.00 & 88.30 & 102.52 & 278.84 & 481.98 & 912.80 & $3,762.93$ \\
\hline Ky. & Wolfe & 52.90 & 166.20 & 64.70 & 37.70 & 0.00 & 0.00 & 0.00 & 0.00 & 0.00 & 0.00 & 321.50 \\
\hline Md. & Allegany & 309.10 & 176.64 & 196.43 & 85.41 & 66.26 & 65.97 & 771.89 & 792.63 & 787.41 & 757.78 & $4,009.52$ \\
\hline Md. & Garrett & $2,817.19$ & $3,462.01$ & $3,675.71$ & $3,014.37$ & $3,201.35$ & $2,884.67$ & $4,332.54$ & $5,051.04$ & $7,507.44$ & $6,522.31$ & $42,468.63$ \\
\hline Ohio & Athens & 0.00 & 0.00 & 0.00 & 0.00 & 0.00 & 0.00 & 5.50 & 0.00 & 46.55 & 52.60 & 104.65 \\
\hline Ohio & Belmont & $6,174.59$ & $6,013.10$ & $5,433.11$ & $5,325.08$ & $5,723.27$ & $6,362.77$ & $6,784.02$ & $6,014.87$ & $5,028.40$ & $4,866.73$ & $57,725.94$ \\
\hline Ohio & Carroll & 0.00 & 68.50 & 0.00 & 2.30 & 0.00 & 0.00 & 112.56 & 103.54 & 115.97 & 118.17 & 521.04 \\
\hline Ohio & Columbiana & 749.53 & 801.93 & 537.71 & 553.99 & 412.67 & 404.44 & 344.04 & 240.07 & 308.43 & 521.40 & $4,874.21$ \\
\hline Ohio & Coshocton & $1,860.90$ & $2,421.70$ & $2,137.60$ & $2,143.20$ & $2,395.50$ & $2,630.30$ & $3,152.80$ & $2,504.50$ & $1,947.00$ & $1,982.80$ & $23,176.30$ \\
\hline Ohio & Gallia & 341.30 & 282.90 & 325.20 & 317.80 & 335.20 & 322.20 & $1,827.50$ & $4,427.30$ & $6,177.50$ & $6,053.50$ & $20,410.40$ \\
\hline Ohio & Guernsey & $1,165.30$ & $1,031.00$ & $1,240.20$ & 0.40 & 0.60 & 13.30 & 0.00 & 0.00 & 0.00 & 0.00 & $3,450.80$ \\
\hline Ohio & Harrison & $1,065.10$ & $1,694.25$ & $1,622.41$ & $1,587.03$ & $1,750.66$ & $1,379.05$ & $1,485.68$ & $2,519.77$ & $2,858.43$ & $2,554.19$ & $18,516.57$ \\
\hline Ohio & Hocking & nd & nd & nd & nd & nd & nd & nd & nd & nd & nd & 0.00 \\
\hline Ohio & Holmes & 114.42 & 161.25 & 178.35 & 143.23 & 135.14 & 136.46 & 41.94 & 0.00 & 0.00 & 0.00 & 910.79 \\
\hline
\end{tabular}


Table 1. Total weight of bituminous coal (in thousands of tons) that was mined in the Appalachian basin for electrical power generation.-Continued [Data are shown by county. Data for 1983 through 1995 from Attanasi and Milici (1998); data for 1996 through 2005 are from the COALdat database. nd, no data]

\begin{tabular}{|c|c|c|c|c|c|c|c|c|c|c|c|c|c|c|c|}
\hline State & County & 1983 & 1984 & 1985 & 1986 & 1987 & 1988 & 1989 & 1990 & 1991 & 1992 & 1993 & 1994 & 1995 & $\begin{array}{l}\text { Total weight } \\
\text { (1983-1995) }\end{array}$ \\
\hline Ohio & Jackson & 226.00 & 316.00 & 338.00 & 678.00 & 696.00 & 654.00 & 887.00 & 988.00 & 789.00 & 586.00 & 928.00 & 918.00 & $1,248.00$ & $9,252.00$ \\
\hline Ohio & Jefferson & $1,576.00$ & $1,679.00$ & $2,363.00$ & $2,836.00$ & $2,547.00$ & $2,387.00$ & $2,196.00$ & $2,258.00$ & $2,118.00$ & $2,294.00$ & $2,451.00$ & $1,102.00$ & 463.00 & $26,270.00$ \\
\hline Ohio & Lawrence & 453.00 & 430.00 & 418.00 & 468.00 & 950.00 & 287.00 & 740.00 & $1,191.00$ & $2,216.00$ & $2,173.00$ & $2,119.00$ & 993.00 & 727.00 & $13,165.00$ \\
\hline Ohio & Mahoning & 179.00 & 135.00 & 128.00 & 228.00 & 250.00 & 201.00 & 236.00 & 147.00 & 79.00 & 21.00 & 46.00 & 34.00 & 10.00 & $1,694.00$ \\
\hline Ohio & Meigs & $3,297.00$ & $4,919.00$ & $5,599.00$ & $4,870.00$ & $5,128.00$ & $4,984.00$ & $3,857.00$ & $5,572.00$ & $5,887.00$ & $5,207.00$ & $3,744.00$ & $4,324.00$ & $4,655.00$ & $62,043.00$ \\
\hline Ohio & Monroe & $2,824.00$ & $3,472.00$ & $3,242.00$ & $3,601.00$ & $3,029.00$ & $2,228.00$ & $1,974.00$ & $1,359.00$ & 766.00 & 106.00 & 657.00 & $1,792.00$ & 570.00 & $25,620.00$ \\
\hline Ohio & Morgan & 504.00 & 318.00 & 386.00 & 0.00 & 0.00 & 0.00 & 118.00 & 0.00 & 0.00 & 0.00 & 0.00 & 0.00 & 0.00 & $1,326.00$ \\
\hline Ohio & Muskingum & $2,959.00$ & $3,182.00$ & $2,995.00$ & $2,636.00$ & 700.00 & 501.00 & 510.00 & 446.00 & 462.00 & 443.00 & 278.00 & 251.00 & 106.00 & $15,469.00$ \\
\hline Ohio & Noble & $1,056.00$ & $1,029.00$ & 831.00 & $1,458.00$ & $3,223.00$ & $2,881.00$ & $3,087.00$ & $2,539.00$ & $3,197.00$ & $3,634.00$ & $3,084.00$ & $2,331.00$ & $1,701.00$ & $30,051.00$ \\
\hline Ohio & Perry & $1,325.00$ & $1,588.00$ & $1,620.00$ & $1,745.00$ & $1,364.00$ & 893.00 & $1,104.00$ & $1,149.00$ & 104.00 & 243.00 & 375.00 & 395.00 & 316.00 & $12,221.00$ \\
\hline Ohio & Pike & 0.00 & 0.00 & 0.00 & 0.00 & 0.00 & 0.00 & 0.00 & 0.00 & 0.00 & 0.00 & 0.00 & 0.00 & 101.00 & 101.00 \\
\hline Ohio & Scioto & 0.00 & 17.00 & 0.00 & 0.00 & 0.00 & 0.00 & 0.00 & 0.00 & 0.00 & 0.00 & 0.00 & 0.00 & 0.00 & 17.00 \\
\hline Ohio & Stark & 203.00 & 224.00 & 229.00 & 280.00 & 195.00 & 125.00 & 13.00 & 24.00 & 19.00 & 2.00 & 48.00 & 0.00 & 0.00 & $1,362.00$ \\
\hline Ohio & Tuscarawas & 273.00 & 895.00 & 755.00 & 710.00 & 786.00 & 706.00 & 595.00 & 591.00 & $1,062.00$ & $1,028.00$ & $1,010.00$ & $1,066.00$ & 774.00 & $10,251.00$ \\
\hline Ohio & Vinton & 152.00 & 244.00 & 322.00 & 339.00 & 330.00 & 319.00 & 956.00 & 388.00 & 286.00 & 414.00 & 453.00 & 582.00 & 401.00 & $5,186.00$ \\
\hline Ohio & Washington & 0.00 & 0.00 & 0.00 & 0.00 & 0.00 & 0.00 & 0.00 & 0.00 & 0.00 & 0.00 & 0.00 & 0.00 & 0.00 & 0.00 \\
\hline $\mathrm{Pa}$. & Allegheny & 684.00 & 650.00 & 625.00 & 951.00 & $1,521.00$ & $1,291.00$ & $1,292.00$ & $1,497.00$ & 834.00 & 589.00 & 178.00 & 17.00 & 0.00 & $10,129.00$ \\
\hline Pa. & Armstrong & $1,473.00$ & $2,069.00$ & $4,065.00$ & $4,225.00$ & $4,228.00$ & $4,728.00$ & $4,575.00$ & $4,397.00$ & $4,097.00$ & $4,894.00$ & $4,059.00$ & $3,786.00$ & $3,736.00$ & $50,332.00$ \\
\hline Pa. & Beaver & 0.00 & 0.00 & 0.00 & 40.00 & 75.00 & 60.00 & 127.00 & 91.00 & 170.00 & 146.00 & 46.00 & 4.00 & 0.00 & 759.00 \\
\hline Pa. & Bedford & 88.00 & 68.00 & 118.00 & 73.00 & 20.00 & 40.00 & 43.00 & 40.00 & 1.00 & 9.00 & 12.00 & 0.00 & 0.00 & 512.00 \\
\hline $\mathrm{Pa}$. & Blair & 0.00 & 0.00 & 0.00 & 0.00 & 0.00 & 0.00 & 0.00 & 0.00 & 0.00 & 10.00 & 1.00 & 0.00 & 0.00 & 11.00 \\
\hline $\mathrm{Pa}$. & Bradford & 122.00 & 0.00 & 0.00 & 0.00 & 0.00 & 0.00 & 0.00 & 0.00 & 0.00 & 0.00 & 0.00 & 0.00 & 0.00 & 122.00 \\
\hline Pa. & Butler & 897.00 & $1,354.00$ & $1,484.00$ & $1,395.00$ & $1,636.00$ & $1,347.00$ & $1,878.00$ & $1,122.00$ & 460.00 & 417.00 & 531.00 & 417.00 & 136.00 & $13,074.00$ \\
\hline Pa. & Cambria & $4,407.00$ & $4,266.00$ & $3,937.00$ & $3,771.00$ & $4,350.00$ & $3,818.00$ & $4,422.00$ & $4,463.00$ & $4,038.00$ & $2,649.00$ & $2,083.00$ & $1,719.00$ & $1,406.00$ & $45,329.00$ \\
\hline Pa. & Cameron & 0.00 & 0.00 & 0.00 & 0.00 & 2.00 & 0.00 & 0.00 & 0.00 & 0.00 & 0.00 & 0.00 & 0.00 & 0.00 & 2.00 \\
\hline Pa. & Centre & 130.00 & 288.00 & 163.00 & 142.00 & 105.00 & 106.00 & 150.00 & 141.00 & 110.00 & 186.00 & 265.00 & 144.00 & 49.00 & $1,979.00$ \\
\hline Pa. & Clarion & $1,658.00$ & $1,465.00$ & $1,058.00$ & $1,292.00$ & $1,696.00$ & $1,654.00$ & $1,825.00$ & $1,325.00$ & $1,089.00$ & 966.00 & 784.00 & 567.00 & 280.00 & $15,659.00$ \\
\hline Pa. & Clearfield & $8,784.00$ & $10,494.00$ & $7,745.00$ & $7,863.00$ & $8,048.00$ & $7,888.00$ & $7,182.00$ & $7,329.00$ & $5,461.00$ & $5,098.00$ & $4,361.00$ & $4,137.00$ & $3,997.00$ & $88,387.00$ \\
\hline Pa. & Clinton & 108.00 & 0.00 & 1.00 & 5.00 & 16.00 & 12.00 & 59.00 & 175.00 & 110.00 & 40.00 & 0.00 & 2.00 & 0.00 & 528.00 \\
\hline Pa. & Elk & 171.00 & 207.00 & 171.00 & 50.00 & 31.00 & 7.00 & 53.00 & 13.00 & 32.00 & 45.00 & 60.00 & 39.00 & 58.00 & 937.00 \\
\hline
\end{tabular}


Table 1. Total weight of bituminous coal (in thousands of tons) that was mined in the Appalachian basin for electrical power generation.-Continued

[Data are shown by county. Data for 1983 through 1995 from Attanasi and Milici (1998); data for 1996 through 2005 are from the COALdat database. nd, no data]

\begin{tabular}{|c|c|c|c|c|c|c|c|c|c|c|c|c|}
\hline State & County & 1996 & 1997 & 1998 & 1999 & 2000 & 2001 & 2002 & 2003 & 2004 & 2005 & $\begin{array}{l}\text { Total weight } \\
\text { (1996-2005) }\end{array}$ \\
\hline Ohio & Jackson & $1,399.30$ & $1,438.40$ & $1,175.20$ & 699.80 & 641.10 & 930.10 & $1,212.86$ & 690.19 & 408.88 & 644.36 & $9,240.19$ \\
\hline Ohio & Jefferson & 234.70 & 189.00 & 340.90 & 246.80 & 335.60 & 330.50 & 501.12 & 433.69 & 542.74 & 478.45 & $3,633.50$ \\
\hline Ohio & Lawrence & 0.00 & 253.10 & 0.00 & 0.00 & 0.00 & 0.00 & 0.00 & 0.00 & 0.00 & 0.00 & 253.10 \\
\hline Ohio & Mahoning & nd & nd & nd & nd & nd & nd & nd & nd & nd & nd & 0.00 \\
\hline Ohio & Meigs & $5,996.60$ & $6,239.80$ & $5,160.60$ & $4,553.60$ & $4,306.50$ & $4,260.10$ & 463.10 & 0.00 & 0.00 & 0.00 & $30,980.30$ \\
\hline Ohio & Monroe & $2,438.60$ & $2,028.40$ & $1,464.00$ & $1,158.20$ & 8.70 & 77.40 & $1,065.60$ & $2,486.52$ & $2,503.86$ & $3,480.13$ & $16,711.41$ \\
\hline Ohio & Morgan & nd & nd & nd & nd & nd & nd & nd & nd & nd & nd & 0.00 \\
\hline Ohio & Muskingum & 94.60 & 126.40 & 199.30 & 100.50 & 312.10 & 533.20 & 192.80 & 0.00 & 0.00 & $1,078.90$ & $2,637.80$ \\
\hline Ohio & Noble & 842.30 & 737.50 & $1,035.58$ & $1,644.30$ & $1,996.80$ & $1,396.85$ & 984.80 & 740.98 & 600.37 & 332.04 & $10,311.52$ \\
\hline Ohio & Perry & 512.10 & 601.57 & 543.60 & 296.30 & 665.90 & 994.20 & 90.60 & 215.79 & $1,000.82$ & 262.94 & $5,183.82$ \\
\hline Ohio & Pike & nd & nd & nd & nd & nd & nd & nd & nd & nd & nd & 0.00 \\
\hline Ohio & Scioto & 0.00 & 0.00 & 0.00 & 0.00 & 0.00 & 0.00 & 0.00 & 0.00 & 29.60 & 0.00 & 29.60 \\
\hline Ohio & Stark & 33.40 & 15.70 & 22.67 & 30.41 & 10.67 & 6.07 & 0.00 & 0.00 & 0.00 & 0.00 & 118.92 \\
\hline Ohio & Tuscarawas & 529.38 & 449.54 & 337.73 & 255.48 & 375.36 & 389.50 & 525.65 & 90.10 & 29.70 & 0.00 & $2,982.44$ \\
\hline Ohio & Vinton & $1,179.60$ & 858.10 & $1,556.80$ & $1,198.00$ & $1,263.50$ & $1,387.50$ & 700.10 & 184.40 & 320.35 & 124.33 & $8,772.68$ \\
\hline Ohio & Washington & 0.00 & 0.00 & 0.00 & 0.00 & 0.00 & 0.00 & 0.00 & $2,410.00$ & $2,409.80$ & $2,473.70$ & $7,293.50$ \\
\hline $\mathrm{Pa}$. & Allegheny & 15.00 & 37.30 & 0.00 & 16.00 & 39.58 & 47.64 & 79.33 & 0.00 & 0.00 & 4.27 & 239.12 \\
\hline Pa. & Armstrong & $4,209.41$ & $3,919.08$ & $4,843.82$ & $4,205.73$ & $2,073.53$ & $2,473.07$ & $6,498.09$ & $8,480.00$ & $8,779.11$ & $8,264.03$ & $53,745.87$ \\
\hline Pa. & Beaver & 0.00 & 0.00 & 0.00 & 16.40 & 131.50 & 0.00 & 22.75 & 10.21 & 209.18 & 349.67 & 739.71 \\
\hline Pa. & Bedford & 0.00 & 0.00 & 0.00 & 0.37 & 24.40 & 0.00 & 0.00 & 7.71 & 0.96 & 0.00 & 33.44 \\
\hline Pa. & Blair & 5.00 & 5.30 & 0.00 & 0.00 & 0.00 & 0.00 & 0.00 & 0.00 & 0.00 & 0.00 & 10.30 \\
\hline $\mathrm{Pa}$. & Bradford & nd & nd & nd & nd & nd & nd & nd & nd & nd & nd & 0.00 \\
\hline $\mathrm{Pa}$. & Butler & 608.13 & $1,241.15$ & $1,400.90$ & 806.95 & 646.32 & 820.77 & 806.62 & 101.55 & 430.68 & 255.88 & $7,118.95$ \\
\hline Pa. & Cambria & $1,240.60$ & $1,057.40$ & $1,092.60$ & 521.30 & 354.13 & 216.50 & $1,084.83$ & 867.38 & $1,811.95$ & $3,835.19$ & $12,081.88$ \\
\hline Pa. & Cameron & nd & nd & nd & nd & nd & nd & nd & nd & nd & nd & 0.00 \\
\hline $\mathrm{Pa}$. & Centre & 53.00 & 36.00 & 18.00 & 0.00 & 0.00 & 0.00 & 0.00 & 28.28 & 0.00 & 0.00 & 135.28 \\
\hline Pa. & Clarion & 114.20 & 173.36 & 128.57 & 207.92 & 125.73 & 87.90 & 57.87 & 31.62 & 20.77 & 172.48 & $1,120.42$ \\
\hline $\mathrm{Pa}$. & Clearfield & $3,776.81$ & $3,408.26$ & $3,264.86$ & $2,335.79$ & $1,771.59$ & $1,308.05$ & $1,996.88$ & $1,900.47$ & $1,562.48$ & $1,512.95$ & $22,838.14$ \\
\hline $\mathrm{Pa}$. & Clinton & nd & nd & nd & nd & nd & nd & nd & nd & nd & nd & 0.00 \\
\hline $\mathrm{Pa}$. & Elk & 52.92 & 63.01 & 38.75 & 170.48 & 20.56 & 74.78 & 112.44 & 42.06 & 418.88 & 281.54 & $1,275.42$ \\
\hline
\end{tabular}


Table 1. Total weight of bituminous coal (in thousands of tons) that was mined in the Appalachian basin for electrical power generation.-Continued [Data are shown by county. Data for 1983 through 1995 from Attanasi and Milici (1998); data for 1996 through 2005 are from the COALdat database. nd, no data]

\begin{tabular}{|c|c|c|c|c|c|c|c|c|c|c|c|c|c|c|c|}
\hline State & County & 1983 & 1984 & 1985 & 1986 & 1987 & 1988 & 1989 & 1990 & 1991 & 1992 & 1993 & 1994 & 1995 & $\begin{array}{l}\text { Total weight } \\
\text { (1983-1995) }\end{array}$ \\
\hline Pa. & Fayette & $1,534.00$ & $1,808.00$ & $2,130.00$ & $2,620.00$ & $2,373.00$ & $2,246.00$ & $1,504.00$ & $1,866.00$ & 273.00 & 574.00 & $1,198.00$ & $1,025.00$ & $1,160.00$ & $20,311.00$ \\
\hline Pa. & Fulton & 9.00 & 15.00 & 25.00 & 18.00 & 12.00 & 11.00 & 12.00 & 8.00 & 8.00 & 13.00 & 5.00 & 0.00 & 3.00 & 139.00 \\
\hline $\mathrm{Pa}$. & Greene & $2,412.00$ & $2,188.00$ & $2,712.00$ & $4,036.00$ & $5,029.00$ & $4,307.00$ & $5,467.00$ & $7,562.00$ & $10,995.00$ & $15,208.00$ & $16,018.00$ & $16,973.00$ & $16,178.00$ & $109,085.00$ \\
\hline Pa. & Huntingdon & 0.00 & 0.00 & 0.00 & 0.00 & 0.00 & 0.00 & 0.00 & 0.00 & 3.00 & 5.00 & 3.00 & 9.00 & 0.00 & 20.00 \\
\hline Pa. & Indiana & $14,502.00$ & $15,109.00$ & $11,770.00$ & $12,125.00$ & $11,469.00$ & $10,161.00$ & $9,972.00$ & $11,310.00$ & $10,769.00$ & $10,095.00$ & $6,354.00$ & $6,940.00$ & $6,057.00$ & $136,633.00$ \\
\hline $\mathrm{Pa}$. & Jefferson & $2,562.00$ & $2,377.00$ & $2,011.00$ & $1,710.00$ & $1,532.00$ & $1,727.00$ & $1,848.00$ & $1,799.00$ & $1,395.00$ & $1,283.00$ & $1,559.00$ & $1,237.00$ & $1,119.00$ & $22,159.00$ \\
\hline Pa. & Lawrence & 380.00 & 318.00 & 294.00 & 430.00 & 423.00 & 220.00 & 169.00 & 254.00 & 108.00 & 47.00 & 31.00 & 0.00 & 78.00 & $2,752.00$ \\
\hline $\mathrm{Pa}$. & Lycoming & 146.00 & 195.00 & 161.00 & 120.00 & 169.00 & 199.00 & 218.00 & 248.00 & 195.00 & 217.00 & 191.00 & 180.00 & 132.00 & $2,371.00$ \\
\hline $\mathrm{Pa}$. & Mercer & 320.00 & 261.00 & 140.00 & 345.00 & 101.00 & 84.00 & 113.00 & 267.00 & 909.00 & 255.00 & 40.00 & 4.00 & 0.00 & $2,839.00$ \\
\hline Pa. & Somerset & $1,674.00$ & $2,057.00$ & $1,579.00$ & $1,818.00$ & $2,284.00$ & $2,287.00$ & $2,533.00$ & $2,829.00$ & $2,788.00$ & $3,922.00$ & $4,023.00$ & $4,270.00$ & $4,661.00$ & $36,725.00$ \\
\hline Pa. & Tioga & 427.00 & 355.00 & 330.00 & 275.00 & 300.00 & 254.00 & 168.00 & 6.00 & 0.00 & 0.00 & 0.00 & 0.00 & 0.00 & $2,115.00$ \\
\hline Pa. & Venango & 428.00 & 642.00 & 488.00 & 580.00 & 603.00 & 484.00 & 623.00 & 632.00 & 322.00 & 16.00 & 0.00 & 0.00 & 0.00 & $4,818.00$ \\
\hline Pa. & Washington & 393.00 & $1,229.00$ & $1,450.00$ & $1,104.00$ & 965.00 & 905.00 & $1,889.00$ & $1,660.00$ & $1,268.00$ & $2,169.00$ & $2,511.00$ & $1,746.00$ & $2,509.00$ & $19,798.00$ \\
\hline $\mathrm{Pa}$. & Westmoreland & 316.00 & 359.00 & 122.00 & 172.00 & 443.00 & 515.00 & 552.00 & 474.00 & 396.00 & 761.00 & 453.00 & 405.00 & 523.00 & $5,491.00$ \\
\hline Tenn. & Anderson & $1,163.00$ & 940.00 & 810.00 & 860.00 & 750.00 & 693.00 & $1,037.00$ & $1,142.00$ & 800.00 & 503.00 & 12.00 & 6.00 & 9.00 & $8,725.00$ \\
\hline Tenn. & Bledsoe & 0.00 & 8.00 & 83.00 & 7.00 & 1.00 & 0.00 & 0.00 & 0.00 & 0.00 & 0.00 & 0.00 & 0.00 & 0.00 & 99.00 \\
\hline Tenn. & Campbell & 773.00 & 652.00 & 898.00 & 985.00 & $1,176.00$ & 620.00 & 339.00 & 752.00 & 928.00 & $1,179.00$ & $1,031.00$ & 504.00 & 433.00 & $10,270.00$ \\
\hline Tenn. & Claiborne & $1,204.00$ & $1,189.00$ & $1,251.00$ & $1,315.00$ & $1,355.00$ & $1,352.00$ & $1,321.00$ & $1,284.00$ & 72.00 & 9.00 & 0.00 & 0.00 & 115.00 & $10,467.00$ \\
\hline Tenn. & Cumberland & 19.00 & 10.00 & 3.00 & 14.00 & 8.00 & 12.00 & 0.00 & 0.00 & 0.00 & 0.00 & 0.00 & 0.00 & 0.00 & 66.00 \\
\hline Tenn. & Fentress & 25.00 & 97.00 & 105.00 & 117.00 & 63.00 & 18.00 & 42.00 & 25.00 & 0.00 & 0.00 & 0.00 & 0.00 & 0.00 & 492.00 \\
\hline Tenn. & Grundy & 35.00 & 42.00 & 71.00 & 51.00 & 25.00 & 21.00 & 19.00 & 39.00 & 27.00 & 0.00 & 0.00 & 0.00 & 0.00 & 330.00 \\
\hline Tenn. & Marion & 0.00 & 0.00 & 12.00 & 136.00 & 32.00 & 62.00 & 398.00 & 27.00 & 0.00 & 0.00 & 0.00 & 0.00 & 0.00 & 667.00 \\
\hline Tenn. & Morgan & 178.00 & 217.00 & 348.00 & 297.00 & 129.00 & 96.00 & 64.00 & 131.00 & 70.00 & 77.00 & 119.00 & 130.00 & 185.00 & $2,041.00$ \\
\hline Tenn. & Rhea & 1.00 & 13.00 & 2.00 & 12.00 & 5.00 & 0.00 & 0.00 & 0.00 & 0.00 & 0.00 & 0.00 & 0.00 & 0.00 & 33.00 \\
\hline Tenn. & Scott & 193.00 & 191.00 & 175.00 & 209.00 & 206.00 & 245.00 & 144.00 & 161.00 & 298.00 & 244.00 & 294.00 & 420.00 & 491.00 & $3,271.00$ \\
\hline Tenn. & Sequatchie & 33.00 & 81.00 & 10.00 & 112.00 & 160.00 & 275.00 & 300.00 & 587.00 & 986.00 & 829.00 & 510.00 & 533.00 & 675.00 & $5,091.00$ \\
\hline Tenn. & Van Buren & 20.00 & 0.00 & 0.00 & 0.00 & 0.00 & 0.00 & 0.00 & 0.00 & 0.00 & 0.00 & 0.00 & 0.00 & 0.00 & 20.00 \\
\hline Va. & Buchanan & $2,194.00$ & $2,837.00$ & $3,193.00$ & $3,686.00$ & $4,393.00$ & $3,688.00$ & $3,523.00$ & $3,094.00$ & $2,310.00$ & $2,457.00$ & $2,097.00$ & $2,649.00$ & $3,431.00$ & $39,552.00$ \\
\hline Va. & Dickenson & $1,635.00$ & 803.00 & 775.00 & 808.00 & 691.00 & 788.00 & 800.00 & 866.00 & $1,485.00$ & $1,759.00$ & $2,014.00$ & $1,576.00$ & $1,152.00$ & $15,152.00$ \\
\hline Va. & Lee & $2,267.00$ & $2,749.00$ & $3,658.00$ & $3,416.00$ & $2,526.00$ & $2,374.00$ & $2,432.00$ & $2,292.00$ & $2,356.00$ & $2,479.00$ & $3,004.00$ & $2,538.00$ & $1,080.00$ & $33,171.00$ \\
\hline
\end{tabular}


Table 1. Total weight of bituminous coal (in thousands of tons) that was mined in the Appalachian basin for electrical power generation.-Continued

[Data are shown by county. Data for 1983 through 1995 from Attanasi and Milici (1998); data for 1996 through 2005 are from the COALdat database. nd, no data]

\begin{tabular}{|c|c|c|c|c|c|c|c|c|c|c|c|c|}
\hline State & County & 1996 & 1997 & 1998 & 1999 & 2000 & 2001 & 2002 & 2003 & 2004 & 2005 & $\begin{array}{l}\text { Total weight } \\
\text { (1996-2005) }\end{array}$ \\
\hline Pa. & Fayette & 905.15 & 712.23 & 807.56 & 481.23 & 444.87 & 454.46 & 85.22 & 79.93 & 205.74 & 509.15 & $4,685.54$ \\
\hline $\mathrm{Pa}$. & Fulton & 8.08 & 0.00 & 0.00 & 0.00 & 0.00 & 0.00 & 0.00 & 0.00 & 0.00 & 0.00 & 8.08 \\
\hline Pa. & Greene & $18,775.22$ & $22,454.05$ & $25,875.71$ & $24,177.75$ & $26,012.28$ & $23,285.67$ & $24,465.22$ & $18,874.68$ & $17,596.01$ & $18,832.57$ & $220,349.16$ \\
\hline $\mathrm{Pa}$. & Huntingdon & nd & nd & nd & nd & nd & nd & nd & $\mathrm{nd}$ & nd & nd & 0.00 \\
\hline Pa. & Indiana & $7,323.50$ & $8,782.20$ & $7,848.41$ & $7,374.68$ & $6,339.58$ & $6,380.56$ & $3,706.52$ & $4,473.44$ & $4,989.60$ & $5,063.95$ & $62,282.44$ \\
\hline Pa. & Jefferson & $1,059.89$ & 727.95 & 761.34 & 355.35 & 122.31 & 60.40 & 268.95 & 321.37 & 203.86 & 352.20 & $4,233.62$ \\
\hline Pa. & Lawrence & 8.80 & 0.00 & 0.00 & 0.00 & 9.00 & 0.00 & 0.00 & 2.79 & 0.00 & 2.79 & 23.38 \\
\hline $\mathrm{Pa}$. & Lycoming & 100.60 & 144.40 & 257.51 & 244.74 & 134.50 & 0.00 & 46.79 & 106.92 & 125.73 & 96.63 & $1,257.82$ \\
\hline Pa. & Mercer & 0.00 & 0.00 & 0.00 & 0.00 & 0.00 & 0.00 & 0.00 & 2.52 & 2.35 & 0.00 & 4.87 \\
\hline Pa. & Somerset & $4,163.79$ & $4,750.30$ & $4,324.69$ & $3,115.77$ & $2,612.33$ & $2,824.21$ & $3,475.13$ & $2,149.02$ & $1,711.56$ & $1,267.46$ & $30,394.26$ \\
\hline Pa. & Tioga & 0.00 & 0.00 & 0.00 & 0.00 & 0.00 & 0.00 & 9.88 & 11.78 & 16.47 & 2.66 & 40.79 \\
\hline $\mathrm{Pa}$. & Venango & 7.53 & 1.60 & 21.80 & 5.20 & 0.00 & 0.00 & 15.24 & 35.04 & 4.00 & 5.96 & 96.37 \\
\hline Pa. & Washington & $3,244.85$ & $4,228.35$ & $6,615.89$ & $5,650.93$ & $4,447.38$ & $3,389.10$ & $4,021.90$ & $4,642.54$ & $4,228.86$ & $4,076.71$ & $44,546.51$ \\
\hline $\mathrm{Pa}$. & Westmoreland & 589.50 & 597.80 & 259.90 & 620.68 & 355.86 & 216.50 & 0.00 & 0.00 & 53.81 & 152.72 & $2,846.77$ \\
\hline Tenn. & Anderson & 668.96 & $1,220.66$ & $1,103.81$ & 366.92 & 0.00 & 0.00 & 0.00 & 16.40 & 160.88 & 33.00 & $3,570.63$ \\
\hline Tenn. & Bledsoe & nd & nd & nd & nd & nd & nd & nd & nd & nd & nd & 0.00 \\
\hline Tenn. & Campbell & 431.87 & 28.01 & 0.00 & 0.00 & 0.00 & 126.42 & 366.98 & 283.97 & 203.24 & 483.59 & $1,924.08$ \\
\hline Tenn. & Claiborne & 378.55 & 438.48 & 358.70 & 457.70 & 562.30 & 875.60 & 890.60 & 822.02 & 992.20 & 993.60 & $6,769.75$ \\
\hline Tenn. & Cumberland & 0.00 & 0.00 & 85.12 & 258.26 & 269.64 & 261.06 & 284.83 & 235.43 & 91.44 & 46.36 & $1,532.14$ \\
\hline Tenn. & Fentress & 0.00 & 0.00 & 0.00 & 0.00 & 0.00 & 0.00 & 0.00 & 0.00 & 8.06 & 0.00 & 8.06 \\
\hline Tenn. & Grundy & 0.00 & 0.00 & 0.00 & 0.00 & 0.00 & 0.00 & 0.00 & 0.00 & 0.00 & 7.20 & 7.20 \\
\hline Tenn. & Marion & nd & nd & nd & nd & nd & nd & nd & nd & nd & nd & 0.00 \\
\hline Tenn. & Morgan & 294.88 & 17.81 & 6.95 & 4.90 & 0.00 & 0.00 & 0.00 & 0.00 & 0.00 & 0.00 & 324.54 \\
\hline Tenn. & Rhea & nd & nd & nd & nd & nd & nd & nd & nd & nd & nd & 0.00 \\
\hline Tenn. & Scott & 473.76 & 400.41 & 327.66 & 457.52 & 351.93 & 142.13 & 0.00 & 0.00 & 26.32 & 132.00 & $2,311.73$ \\
\hline Tenn. & Sequatchie & 691.30 & 943.59 & 552.73 & 429.14 & 402.97 & 0.00 & 0.00 & 0.00 & 0.00 & 0.00 & $3,019.73$ \\
\hline Tenn. & Van Buren & nd & nd & nd & $\mathrm{nd}$ & $\mathrm{nd}$ & nd & nd & nd & nd & nd & 0.00 \\
\hline Va. & Buchanan & $3,125.36$ & $2,797.43$ & $2,727.16$ & $3,043.71$ & $2,216.09$ & $2,768.20$ & $3,490.85$ & $2,945.61$ & $1,637.65$ & $1,727.81$ & $26,479.87$ \\
\hline Va. & Dickenson & $1,601.36$ & 917.87 & $1,126.46$ & $1,788.01$ & $1,909.02$ & 816.21 & 423.23 & $1,040.04$ & 593.92 & 756.91 & $10,973.03$ \\
\hline Va. & Lee & $1,782.14$ & $2,419.27$ & $2,386.46$ & $2,997.87$ & $3,614.65$ & $5,255.99$ & $5,199.24$ & $5,418.30$ & $2,291.77$ & $1,757.00$ & $33,122.69$ \\
\hline
\end{tabular}


Table 1. Total weight of bituminous coal (in thousands of tons) that was mined in the Appalachian basin for electrical power generation.-Continued [Data are shown by county. Data for 1983 through 1995 from Attanasi and Milici (1998); data for 1996 through 2005 are from the COALdat database. nd, no data]

\begin{tabular}{|c|c|c|c|c|c|c|c|c|c|c|c|c|c|c|c|}
\hline State & County & 1983 & 1984 & 1985 & 1986 & 1987 & 1988 & 1989 & 1990 & 1991 & 1992 & 1993 & 1994 & 1995 & $\begin{array}{l}\text { Total weight } \\
\text { (1983-1995) }\end{array}$ \\
\hline Va. & Russell & $1,562.00$ & $1,876.00$ & $1,295.00$ & $1,367.00$ & $1,375.00$ & $1,488.00$ & $1,445.00$ & $1,774.00$ & $1,070.00$ & 619.00 & 462.00 & 787.00 & 799.00 & $15,919.00$ \\
\hline Va. & Scott & 0.00 & 0.00 & 0.00 & 2.00 & 0.00 & 0.00 & 0.00 & 0.00 & 0.00 & 0.00 & 0.00 & 0.00 & 0.00 & 2.00 \\
\hline Va. & Tazewell & 71.00 & 75.00 & 54.00 & 70.00 & 84.00 & 33.00 & 44.00 & 7.00 & 0.00 & 0.00 & 0.00 & 0.00 & 0.00 & 438.00 \\
\hline Va. & Wise & $6,081.00$ & $7,515.00$ & $7,945.00$ & $8,386.00$ & $9,850.00$ & $9,686.00$ & $9,741.00$ & $9,299.00$ & $8,935.00$ & $9,195.00$ & $9,050.00$ & $8,717.00$ & $7,989.00$ & $112,389.00$ \\
\hline W. Va. & Barbour & $2,388.00$ & $3,190.00$ & $2,492.00$ & $1,428.00$ & $1,528.00$ & $1,934.00$ & $1,905.00$ & $2,046.00$ & $2,059.00$ & $2,628.00$ & $2,830.00$ & $2,669.00$ & $1,074.00$ & $28,171.00$ \\
\hline W. Va. & Boone & $9,403.00$ & $10,007.00$ & $10,561.00$ & $13,014.00$ & $14,606.00$ & $13,480.00$ & $15,935.00$ & $16,711.00$ & $15,408.00$ & $14,208.00$ & $9,764.00$ & $14,343.00$ & $14,112.00$ & $171,552.00$ \\
\hline W. Va. & Braxton & 392.00 & 389.00 & 208.00 & 189.00 & 296.00 & 296.00 & 68.00 & 63.00 & 6.00 & 0.00 & 146.00 & 0.00 & 0.00 & $2,053.00$ \\
\hline W. Va. & Brooke & $1,040.00$ & $1,288.00$ & 990.00 & $1,014.00$ & $1,047.00$ & 833.00 & $1,188.00$ & $1,572.00$ & $1,505.00$ & $1,669.00$ & $1,694.00$ & $1,232.00$ & $1,049.00$ & $16,121.00$ \\
\hline W. Va. & Clay & 0.00 & 25.00 & 0.00 & 1.00 & 92.00 & 330.00 & 707.00 & 524.00 & 271.00 & 300.00 & 664.00 & 878.00 & 979.00 & $4,771.00$ \\
\hline W. Va. & Fayette & 391.00 & 366.00 & 322.00 & 405.00 & 491.00 & $1,425.00$ & $1,850.00$ & $2,088.00$ & $2,011.00$ & $1,930.00$ & $1,878.00$ & $2,142.00$ & $1,651.00$ & $16,950.00$ \\
\hline W. Va. & Gilmer & 5.00 & 5.00 & 0.00 & 0.00 & 38.00 & 8.00 & 0.00 & 0.00 & 0.00 & 0.00 & 0.00 & 0.00 & 0.00 & 56.00 \\
\hline W. Va. & Grant & $3,224.00$ & $3,678.00$ & $3,363.00$ & $3,110.00$ & $3,145.00$ & $3,368.00$ & $4,138.00$ & $4,117.00$ & $4,117.00$ & $3,959.00$ & $2,732.00$ & $2,853.00$ & $3,052.00$ & $44,856.00$ \\
\hline W. Va. & Greenbrier & 53.00 & 63.00 & 96.00 & 50.00 & 57.00 & 13.00 & 3.00 & 0.00 & 2.00 & 11.00 & 6.00 & 4.00 & 21.00 & 379.00 \\
\hline W. Va. & Harrison & $2,715.00$ & $2,782.00$ & $3,045.00$ & $3,353.00$ & $3,781.00$ & $3,353.00$ & $4,303.00$ & $4,369.00$ & $4,103.00$ & $3,901.00$ & $2,258.00$ & $4,124.00$ & $4,536.00$ & $46,623.00$ \\
\hline W. Va. & Kanawha & $5,618.00$ & $5,540.00$ & $4,324.00$ & $4,646.00$ & $3,957.00$ & $3,464.00$ & $4,332.00$ & $5,090.00$ & $5,582.00$ & $5,793.00$ & $5,912.00$ & $6,848.00$ & $8,159.00$ & $69,265.00$ \\
\hline W. Va. & Lewis & $1,191.00$ & $1,036.00$ & $1,059.00$ & $1,012.00$ & 665.00 & 565.00 & 192.00 & 48.00 & 0.00 & 0.00 & 2.00 & 56.00 & 30.00 & $5,856.00$ \\
\hline W. Va. & Lincoln & 0.00 & 0.00 & 0.00 & 0.00 & 22.00 & 31.00 & 45.00 & 24.00 & 66.00 & $1,456.00$ & $1,828.00$ & $1,335.00$ & 946.00 & $5,753.00$ \\
\hline W. Va. & Logan & $4,608.00$ & $6,008.00$ & $5,412.00$ & $8,060.00$ & $8,707.00$ & $8,649.00$ & $5,042.00$ & $7,349.00$ & $7,113.00$ & $6,840.00$ & $6,613.00$ & $8,917.00$ & $9,967.00$ & $93,285.00$ \\
\hline W. Va. & McDowell & 47.00 & 119.00 & 11.00 & 41.00 & 239.00 & 74.00 & 67.00 & 25.00 & 80.00 & 40.00 & 20.00 & 17.00 & 19.00 & 799.00 \\
\hline W. Va. & Marion & $9,342.00$ & $4,946.00$ & $4,593.00$ & $4,065.00$ & $4,527.00$ & $3,856.00$ & $3,706.00$ & $4,652.00$ & $3,885.00$ & $1,981.00$ & $1,977.00$ & $1,998.00$ & $1,272.00$ & $50,800.00$ \\
\hline W. Va. & Marshall & $5,281.00$ & $5,050.00$ & $4,583.00$ & $4,322.00$ & $5,723.00$ & $6,451.00$ & $6,700.00$ & $7,920.00$ & $8,165.00$ & $8,591.00$ & $4,366.00$ & $7,122.00$ & $8,249.00$ & $82,523.00$ \\
\hline W. Va. & Mason & 47.00 & 0.00 & 0.00 & 0.00 & 107.00 & 52.00 & 37.00 & 73.00 & 113.00 & 12.00 & 42.00 & 41.00 & 0.00 & 524.00 \\
\hline W. Va. & Mercer & 0.00 & 0.00 & 67.00 & 27.00 & 66.00 & 0.00 & 0.00 & 0.00 & 0.00 & 0.00 & 0.00 & 0.00 & 0.00 & 160.00 \\
\hline W. Va. & Mineral & 40.00 & 176.00 & 39.00 & 84.00 & 77.00 & 167.00 & 240.00 & 98.00 & 145.00 & 248.00 & 219.00 & 197.00 & 53.00 & $1,783.00$ \\
\hline W. Va. & Mingo & $5,590.00$ & $5,985.00$ & $5,097.00$ & $6,370.00$ & $7,329.00$ & $8,710.00$ & $8,929.00$ & $10,028.00$ & $12,468.00$ & $13,681.00$ & $15,145.00$ & $16,280.00$ & $13,887.00$ & $129,499.00$ \\
\hline W. Va. & Monongalia & $6,340.00$ & $13,080.00$ & $8,541.00$ & $9,682.00$ & $12,393.00$ & $11,424.00$ & $12,453.00$ & $12,624.00$ & $10,785.00$ & $11,127.00$ & $8,630.00$ & $11,734.00$ & $11,343.00$ & $140,156.00$ \\
\hline W. Va. & Nicholas & $1,499.00$ & $2,583.00$ & $2,482.00$ & $3,649.00$ & $4,472.00$ & $4,133.00$ & $3,268.00$ & $2,662.00$ & $1,922.00$ & $1,175.00$ & $1,155.00$ & $1,729.00$ & $1,655.00$ & $32,384.00$ \\
\hline W. Va. & Ohio & 62.00 & 0.00 & 435.00 & 163.00 & 85.00 & 15.00 & 286.00 & 255.00 & 94.00 & 162.00 & 495.00 & $1,029.00$ & 108.00 & $3,189.00$ \\
\hline W. Va. & Preston & $2,787.00$ & $4,096.00$ & $3,362.00$ & $3,053.00$ & $3,637.00$ & $3,599.00$ & $3,580.00$ & $3,144.00$ & $2,500.00$ & $2,093.00$ & $1,695.00$ & $1,940.00$ & $1,442.00$ & $36,928.00$ \\
\hline W. Va. & Raleigh & 70.00 & 31.00 & 73.00 & 77.00 & 0.00 & 0.00 & 10.00 & 45.00 & 68.00 & 250.00 & 10.00 & 0.00 & 0.00 & 634.00 \\
\hline
\end{tabular}


Table 1. Total weight of bituminous coal (in thousands of tons) that was mined in the Appalachian basin for electrical power generation.-Continued [Data are shown by county. Data for 1983 through 1995 from Attanasi and Milici (1998); data for 1996 through 2005 are from the COALdat database. nd, no data]

\begin{tabular}{|c|c|c|c|c|c|c|c|c|c|c|c|c|}
\hline State & County & 1996 & 1997 & 1998 & 1999 & 2000 & 2001 & 2002 & 2003 & 2004 & 2005 & $\begin{array}{l}\text { Total weight } \\
\text { (1996-2005) }\end{array}$ \\
\hline Va. & Russell & 615.14 & $1,251.80$ & $1,326.64$ & $1,440.34$ & $1,799.26$ & $2,081.82$ & $1,379.80$ & $1,515.48$ & $1,250.02$ & $1,069.20$ & $13,729.50$ \\
\hline Va. & Scott & nd & nd & nd & $\mathrm{nd}$ & nd & nd & nd & $\mathrm{nd}$ & nd & nd & 0.00 \\
\hline Va. & Tazewell & 14.84 & 0.00 & 0.00 & 0.00 & 0.00 & 0.00 & 131.00 & 85.43 & 21.21 & 185.49 & 437.97 \\
\hline Va. & Wise & $7,313.16$ & $7,894.78$ & $9,436.11$ & $10,501.09$ & $9,761.55$ & $10,463.30$ & $11,269.57$ & $12,179.83$ & $12,450.29$ & $11,257.39$ & $102,527.07$ \\
\hline W. Va. & Barbour & $1,430.68$ & $1,401.22$ & $1,340.81$ & $1,316.71$ & 587.08 & 419.48 & $1,088.98$ & 577.23 & 554.19 & 218.70 & $8,935.08$ \\
\hline W. Va. & Boone & $15,805.31$ & $16,591.65$ & $19,405.75$ & $21,413.87$ & $19,642.15$ & $20,259.40$ & $22,345.84$ & $17,991.56$ & $17,390.87$ & $17,530.85$ & $188,377.25$ \\
\hline W. Va. & Braxton & nd & nd & nd & nd & nd & nd & $\mathrm{nd}$ & $\mathrm{nd}$ & nd & $\mathrm{nd}$ & 0.00 \\
\hline W. Va. & Brooke & $1,034.30$ & $1,520.80$ & $1,858.60$ & $1,630.80$ & $1,373.10$ & 813.64 & 368.10 & 0.00 & 0.00 & 0.00 & $8,599.34$ \\
\hline W. Va. & Clay & $2,351.83$ & $3,566.51$ & $4,405.73$ & $4,512.39$ & $3,718.98$ & $3,352.04$ & $4,656.03$ & $3,660.15$ & $3,170.09$ & $2,667.93$ & $36,061.68$ \\
\hline W. Va. & Fayette & $1,989.50$ & $2,324.61$ & $1,715.09$ & $1,268.36$ & 989.34 & $1,074.72$ & 301.76 & 426.72 & 51.42 & 514.05 & $10,655.57$ \\
\hline W. Va. & Gilmer & nd & nd & nd & nd & nd & nd & nd & nd & nd & nd & 0.00 \\
\hline W. Va. & Grant & $3,597.31$ & $1,579.53$ & $1,319.60$ & $1,146.89$ & $1,048.01$ & 705.95 & 608.64 & 811.04 & 516.57 & 46.48 & $11,380.02$ \\
\hline W. Va. & Greenbrier & 0.00 & 0.00 & 0.00 & 57.62 & 240.08 & 273.59 & 281.32 & 6.82 & 0.00 & 0.00 & 859.43 \\
\hline W. Va. & Harrison & $5,322.42$ & $5,699.56$ & $6,362.78$ & $6,051.66$ & $6,346.36$ & $4,659.20$ & $5,926.26$ & $5,142.09$ & $5,792.22$ & $4,924.53$ & $56,227.08$ \\
\hline W. Va. & Kanawha & $10,917.43$ & $10,552.34$ & $13,001.72$ & $12,986.05$ & $11,792.65$ & $8,570.06$ & $12,640.36$ & $13,655.55$ & $11,962.58$ & $12,370.68$ & $118,449.42$ \\
\hline W. Va. & Lewis & nd & nd & nd & nd & nd & nd & nd & nd & nd & nd & 0.00 \\
\hline W. Va. & Lincoln & 54.30 & 29.80 & 102.90 & 1.60 & 259.77 & $1,307.46$ & 221.38 & 106.09 & 288.26 & 788.92 & $3,160.48$ \\
\hline W. Va. & Logan & $12,043.56$ & $11,417.21$ & $7,774.41$ & $4,786.92$ & $3,819.52$ & $3,526.70$ & $6,620.69$ & $6,381.00$ & $5,177.26$ & $5,785.50$ & $67,332.77$ \\
\hline W. Va. & McDowell & 10.70 & 0.00 & 0.00 & 41.60 & 291.89 & 624.91 & $1,063.62$ & 771.00 & 464.09 & 188.14 & $3,455.95$ \\
\hline W. Va. & Marion & $1,073.65$ & $2,601.98$ & $2,858.67$ & 968.14 & 0.00 & 338.94 & 28.82 & 465.67 & $1,926.86$ & $3,450.46$ & $13,713.19$ \\
\hline W. Va. & Marshall & $9,460.74$ & $11,009.08$ & $12,004.55$ & $10,698.35$ & $8,742.01$ & $7,610.70$ & $5,711.08$ & $6,278.32$ & $7,081.14$ & $8,025.50$ & $86,621.47$ \\
\hline W. Va. & Mason & 0.00 & 0.00 & 0.00 & 0.00 & 49.00 & 0.00 & 0.00 & 0.00 & 23.81 & 0.00 & 72.81 \\
\hline W. Va. & Mercer & 0.00 & 0.00 & 0.00 & 0.00 & 0.00 & 0.00 & 189.00 & 0.00 & 0.00 & 0.00 & 189.00 \\
\hline W. Va. & Mineral & 57.41 & 0.00 & 0.00 & 0.00 & 6.46 & 0.00 & 0.00 & 0.00 & 0.00 & 8.59 & 72.46 \\
\hline W. Va. & Mingo & $15,578.28$ & $15,780.88$ & $15,047.33$ & $14,232.95$ & $12,143.20$ & $15,068.58$ & $13,517.08$ & $13,063.86$ & $9,766.25$ & $9,253.90$ & $133,452.31$ \\
\hline W. Va. & Monongalia & $11,183.44$ & $9,289.71$ & $7,955.86$ & $8,733.56$ & $7,219.10$ & $7,306.60$ & $8,515.11$ & $5,621.55$ & $6,807.78$ & $7,167.00$ & $79,799.71$ \\
\hline W. Va. & Nicholas & 849.09 & 613.22 & 189.53 & $1,515.56$ & $3,417.99$ & $5,322.29$ & $4,001.05$ & $4,490.34$ & $3,911.82$ & $4,220.46$ & $28,531.35$ \\
\hline W. Va. & Ohio & 0.00 & 0.00 & 0.00 & 0.00 & 91.70 & 0.00 & 0.00 & 0.00 & 0.00 & 0.00 & 91.70 \\
\hline W. Va. & Preston & $1,679.62$ & $1,481.96$ & $1,374.09$ & $1,259.18$ & $1,244.28$ & $1,072.50$ & $2,280.97$ & $1,342.27$ & $1,510.85$ & $1,280.85$ & $14,526.57$ \\
\hline W. Va. & Raleigh & 92.90 & 322.43 & 458.91 & 285.50 & 62.10 & 218.10 & 394.45 & 312.61 & 468.55 & 848.80 & $3,464.35$ \\
\hline
\end{tabular}


Table 1. Total weight of bituminous coal (in thousands of tons) that was mined in the Appalachian basin for electrical power generation.-Continued

[Data are shown by county. Data for 1983 through 1995 from Attanasi and Milici (1998); data for 1996 through 2005 are from the COALdat database. nd, no data]

\begin{tabular}{|c|c|c|c|c|c|c|c|c|c|c|c|c|c|c|c|}
\hline State & County & 1983 & 1984 & 1985 & 1986 & 1987 & 1988 & 1989 & 1990 & 1991 & 1992 & 1993 & 1994 & 1995 & $\begin{array}{c}\text { Total weight } \\
\text { (1983-1995) }\end{array}$ \\
\hline W. Va. & Randolph & 677.00 & 861.00 & 990.00 & $1,215.00$ & $1,150.00$ & 989.00 & 868.00 & 551.00 & 234.00 & 52.00 & 81.00 & 37.00 & 10.00 & $7,715.00$ \\
\hline W. Va. & Taylor & 0.00 & 0.00 & 14.00 & 8.00 & 0.00 & 7.00 & 7.00 & 7.00 & 0.00 & 17.00 & 1.00 & 0.00 & 0.00 & 61.00 \\
\hline W. Va. & Tucker & 5.00 & 91.00 & 39.00 & 62.00 & 0.00 & 0.00 & 0.00 & 0.00 & 0.00 & 0.00 & 0.00 & 0.00 & 0.00 & 197.00 \\
\hline W. Va. & Upshur & $1,442.00$ & 983.00 & 866.00 & 439.00 & 260.00 & 188.00 & 185.00 & 98.00 & 40.00 & 269.00 & 533.00 & 330.00 & 472.00 & $6,105.00$ \\
\hline W. Va. & Wayne & 88.00 & 0.00 & 1.00 & 172.00 & 95.00 & 84.00 & 147.00 & 202.00 & 549.00 & $1,109.00$ & $2,346.00$ & $2,491.00$ & $3,866.00$ & $11,150.00$ \\
\hline W. Va. & Webster & 129.00 & 342.00 & 647.00 & 911.00 & $1,092.00$ & $1,426.00$ & $1,822.00$ & $1,670.00$ & $1,032.00$ & $1,248.00$ & $2,026.00$ & $2,102.00$ & $2,582.00$ & $17,029.00$ \\
\hline W. Va. & Wyoming & 869.00 & 476.00 & 743.00 & 374.00 & 318.00 & 317.00 & 202.00 & 0.00 & 0.00 & 0.00 & 43.00 & 40.00 & 24.00 & $3,406.00$ \\
\hline
\end{tabular}


Table 1. Total weight of bituminous coal (in thousands of tons) that was mined in the Appalachian basin for electrical power generation.-Continued [Data are shown by county. Data for 1983 through 1995 from Attanasi and Milici (1998); data for 1996 through 2005 are from the COALdat database. nd, no data]

\begin{tabular}{|c|c|c|c|c|c|c|c|c|c|c|c|c|}
\hline State & County & 1996 & 1997 & 1998 & 1999 & 2000 & 2001 & 2002 & 2003 & 2004 & 2005 & $\begin{array}{l}\text { Total weight } \\
\text { (1996-2005) }\end{array}$ \\
\hline W. Va. & Randolph & 0.00 & 0.00 & 0.00 & 0.00 & 0.00 & 0.00 & 0.00 & 0.55 & 0.00 & 0.00 & 0.55 \\
\hline W. Va. & Taylor & nd & nd & nd & nd & nd & nd & nd & nd & nd & nd & 0.00 \\
\hline W. Va. & Tucker & nd & nd & nd & nd & nd & nd & nd & nd & nd & nd & 0.00 \\
\hline W. Va. & Upshur & 775.65 & 761.17 & $1,078.08$ & $1,633.15$ & $2,541.44$ & $2,426.07$ & $1,408.77$ & $1,205.42$ & 844.20 & 890.47 & $13,564.42$ \\
\hline W. Va. & Wayne & $4,056.42$ & $4,525.65$ & $5,276.40$ & $6,398.99$ & $5,720.36$ & $4,729.62$ & $3,599.59$ & $3,915.70$ & $5,172.62$ & $6,087.81$ & $49,483.16$ \\
\hline W. Va. & Webster & $3,007.49$ & $3,507.67$ & $3,704.58$ & $4,431.69$ & $4,363.27$ & $4,274.94$ & $4,036.61$ & $3,660.83$ & $2,239.05$ & $2,553.30$ & $35,779.43$ \\
\hline W. Va. & Wyoming & 56.48 & 186.50 & 455.04 & 307.57 & 273.43 & 791.01 & 874.57 & 915.27 & 539.25 & 855.46 & $5,254.58$ \\
\hline
\end{tabular}


Table 2. Heating values of bituminous coal (in British thermal units per pound) that was mined in the Appalachian basin for electrical power generation. [Data are shown by county. Data for 1983 through 1995 are from Attanasi and Milici (1998); data for 1996 through 2005 are from the COALdat database. nd, no data]

\begin{tabular}{|c|c|c|c|c|c|c|c|c|c|c|c|c|c|c|c|c|c|c|c|c|c|c|c|c|}
\hline State & County & 1983 & 1984 & 1985 & 1986 & 1987 & 1988 & 1989 & 1990 & 1991 & 1992 & 1993 & 1994 & 1995 & 1996 & 1997 & 1998 & 1999 & 2000 & 2001 & 2002 & 2003 & 2004 & 2005 \\
\hline Ala. & Bibb & 0 & 0 & 0 & 0 & 0 & 0 & 0 & 0 & 0 & 0 & 0 & 0 & 10,263 & 11,820 & 11,742 & 12,340 & 0 & 0 & 0 & 0 & 0 & 11,775 & 12,179 \\
\hline Ala. & Blount & 0 & 12,309 & 12,246 & 12,452 & 12,897 & 13,040 & 0 & 11,979 & 12,050 & 0 & 0 & 0 & 0 & nd & nd & nd & nd & nd & nd & nd & nd & nd & nd \\
\hline Ala. & Cullman & 13,295 & 13,312 & 0 & 12,417 & 0 & 0 & 0 & 0 & 0 & 0 & 0 & 0 & 0 & nd & nd & nd & nd & nd & nd & nd & nd & nd & nd \\
\hline Ala. & De Kalb & 0 & 0 & 0 & 12,631 & 0 & 0 & 0 & 0 & 0 & 0 & 0 & 0 & 0 & nd & nd & nd & nd & nd & nd & nd & nd & nd & nd \\
\hline Ala. & Fayette & 12,042 & 12,043 & 12,099 & 12,211 & 12,201 & 12,125 & 12,075 & 12,060 & 11,999 & 12,049 & 12,015 & 11,994 & 12,089 & 12,169 & 0 & 0 & 0 & 0 & 12,015 & 12,152 & 0 & 0 & 0 \\
\hline
\end{tabular}

Ala.

Ala

Ala.

Ala.

Ala

Ala.

Ala.

Ala.

Ala.

Ala.

Ga.

Ky.

Ky.

Ky.

Ky.

Ky.

Ky.

Ky.

Ky.

Ky.

Ky.

Ky.

Ky.

Ky.

Ky.

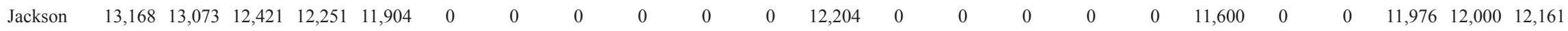
$\begin{array}{lllllllllllllllllllllllll}\text { Jefferson } & 12,414 & 12,389 & 12,470 & 12,466 & 12,573 & 12,478 & 12,395 & 12,397 & 12,312 & 12,210 & 12,339 & 12,377 & 12,463 & 12,413 & 12,315 & 12,351 & 12,306 & 12,392 & 12,265 & 12,247 & 12,177 & 12,155 & 12,142\end{array}$

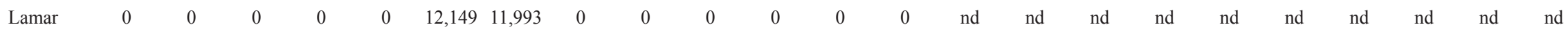

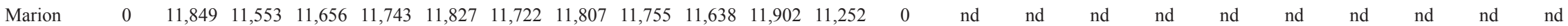

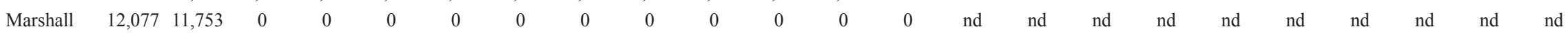

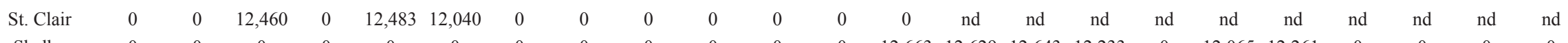
$\begin{array}{rrrrrrrrrrrrrrrrrrrrrrrrrrrrrr}\text { Shelby } & 0 & 0 & 0 & 0 & 0 & 0 & 0 & 0 & 0 & 0 & 0 & 0 & 12,663 & 12,629 & 12,643 & 12,233 & 0 & 12,065 & 12,261 & 0 & 0 & 0 & 0\end{array}$

$\begin{array}{llllllllllllllllllllllllll}\text { Tuscaloosa } & 12,057 & 12,491 & 12,209 & 12,499 & 12,614 & 12,612 & 12,446 & 12,439 & 12,325 & 12,276 & 12,378 & 12,358 & 12,435 & 12,319 & 12,306 & 12,495 & 12,355 & 12,225 & 12,095 & 12,265 & 12,219 & 12,159 & 12,205\end{array}$ $\begin{array}{llllllllllllllllllllllll}\text { Walker } & 11,934 & 11,966 & 11,998 & 12,127 & 12,123 & 12,021 & 11,934 & 11,999 & 11,996 & 11,941 & 11,930 & 12,027 & 12,126 & 12,104 & 12,139 & 12,138 & 11,902 & 11,851 & 11,843 & 11,890 & 11,792 & 11,588 & 11,725\end{array}$

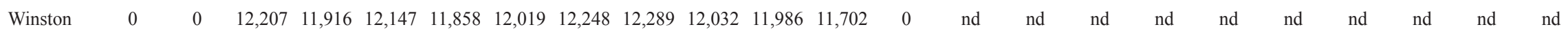

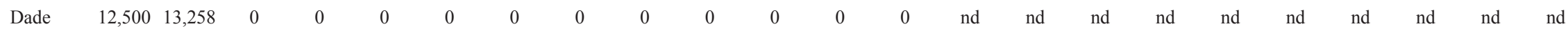

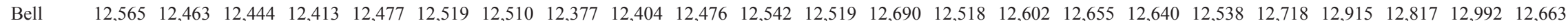

$\begin{array}{llllllllllllllllllllllllll}\text { Boyd } & 11,420 & 11,461 & 11,357 & 11,447 & 11,616 & 12,237 & 11,997 & 12,682 & 12,573 & 12,583 & 12,534 & 12,391 & 12,519 & 12,468 & 12,247 & 11,999 & 12,348 & 12,118 & 11,925 & 12,104 & 12,044 & 12,061 & 11,790\end{array}$ $\begin{array}{lllllllllllllllllllllllllllllllll}\text { Breathitt } & 11,892 & 11,628 & 11,523 & 11,601 & 11,806 & 11,908 & 11,705 & 11,823 & 12,135 & 12,080 & 12,098 & 12,062 & 12,042 & 12,032 & 12,188 & 12,001 & 11,997 & 12,336 & 12,041 & 12,314 & 12,252 & 12,186 & 12,416\end{array}$

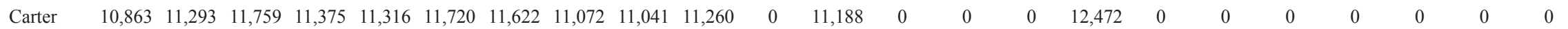

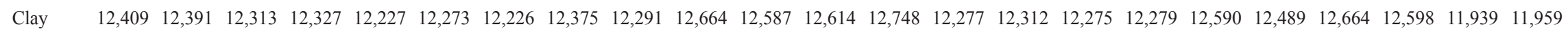

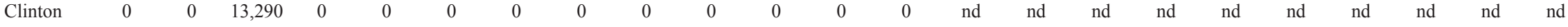

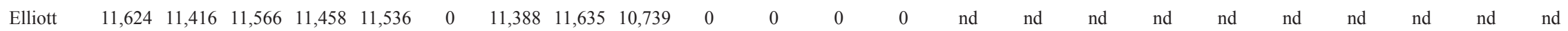
$\begin{array}{llllllllllllllllllllllll}\text { Estill } & 12,025 & 0 & 0 & 12,895 & 0 & 0 & 0 & 0 & 0 & 0 & 12,423 & 11,994 & 12,862 & 12,500 & 12,821 & 0 & 12,577 & 11,697 & 11,929 & 11,968 & 12,106 & 12,171 & 0\end{array}$

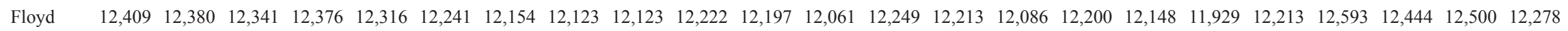

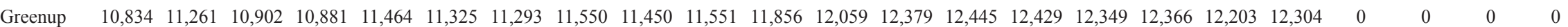
$\begin{array}{lllllllllllllllllllllllllllll}\text { Harlan } & 12,244 & 12,310 & 12,528 & 12,631 & 12,657 & 12,659 & 12,548 & 12,590 & 12,685 & 12,767 & 12,655 & 12,662 & 12,717 & 12,732 & 12,804 & 12,790 & 12,816 & 12,786 & 12,712 & 12,670 & 12,704 & 12,584 & 12,599\end{array}$

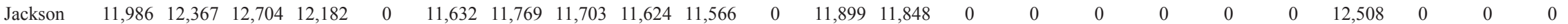
$\begin{array}{llllllllllllllllllllllllllll}\text { Johnson } & 11,782 & 11,670 & 11,686 & 11,859 & 11,925 & 11,963 & 11,668 & 11,829 & 11,778 & 11,852 & 11,795 & 11,743 & 11,942 & 11,947 & 11,560 & 11,932 & 12,049 & 12,058 & 11,881 & 11,949 & 11,928 & 11,899 & 11,463\end{array}$ $\begin{array}{llllllllllllllllllllllllll}\text { Knott } & 12,081 & 12,513 & 12,459 & 12,575 & 12,713 & 12,428 & 12,297 & 12,310 & 12,554 & 12,520 & 12,478 & 12,428 & 12,394 & 12,378 & 12,348 & 12,410 & 12,497 & 12,564 & 12,465 & 12,415 & 12,535 & 12,401 & 12,402\end{array}$ 
Table 2. Heating values of bituminous coal (in British thermal units per pound) that was mined in the Appalachian basin for electrical power generation.-Continued

[Data are shown by county. Data for 1983 through 1995 are from Attanasi and Milici (1998); data for 1996 through 2005 are from the COALdat database. nd, no data]

\begin{tabular}{|c|c|c|c|c|c|c|c|c|c|c|c|c|c|c|c|c|c|c|c|c|c|c|c|c|}
\hline State & County & 1983 & 1984 & 1985 & 1986 & 1987 & 1988 & 1989 & 1990 & 1991 & 1992 & 1993 & 1994 & 1995 & 1996 & 1997 & 1998 & 1999 & 2000 & 2001 & 2002 & 2003 & 2004 & 2005 \\
\hline Ky. & Knox & 13,180 & 12,758 & 12,494 & 12,401 & 12,230 & 12,232 & 12,258 & 11,292 & 13,120 & 12,901 & 12,987 & 11,812 & 12,352 & 0 & 0 & 11,556 & 0 & 0 & 0 & 11,934 & 12,353 & 12,014 & 11,905 \\
\hline Ky. & Laurel & 12,075 & 12,061 & 12,212 & 12,150 & 12,140 & 12,347 & 12,151 & 12,188 & 11,857 & 11,985 & 12,616 & 0 & 11,145 & 12,417 & 0 & 11,808 & 11,552 & 11,625 & 11,033 & 0 & 11,607 & 12,715 & 0 \\
\hline Ку. & Lawrence & 11,245 & 10,975 & 11,861 & 11,466 & 11,653 & 11,676 & 11,583 & 11,218 & 11,319 & 11,684 & 11,598 & 11,675 & 11,745 & 11,323 & 11,383 & 11,598 & 12,300 & 11,863 & 11,875 & 11,865 & 11,490 & 11,720 & 11,795 \\
\hline Ky. & Lee & 0 & 0 & 11,963 & 11,984 & 11,959 & 12,168 & 11,405 & 11,419 & 0 & 0 & 11,117 & 0 & 11,974 & 0 & 0 & 0 & 0 & 0 & 0 & 11,893 & 0 & 0 & 0 \\
\hline Ky. & Leslie & 12,084 & 12,038 & 12,223 & 12,447 & 12,469 & 12,353 & 12,306 & 12,281 & 12,391 & 12,508 & 12,391 & 12,460 & 12,500 & 12,670 & 12,786 & 12,564 & 12,492 & 12,584 & 12,297 & 12,254 & 12,244 & 11,940 & 11,873 \\
\hline Ку. & Letcher & 12,655 & 12,793 & 12,845 & 12,972 & 12,960 & 12,961 & 12,820 & 12,857 & 12,755 & 12,749 & 12,849 & 12,872 & 12,983 & 12,980 & 12,991 & 13,031 & 12,969 & 12,756 & 12,724 & 12,741 & 12,822 & 12,640 & 12,724 \\
\hline Ky. & McCreary & 12,319 & 12,490 & 12,356 & 12,383 & 12,556 & 12,425 & 0 & 0 & 0 & 13,250 & 12,886 & 13,017 & 13,167 & 13,121 & 0 & 0 & 0 & 0 & 0 & 0 & 0 & 0 & 0 \\
\hline Ky. & Magoffin & 12,126 & 12,021 & 11,715 & 11,925 & 11,945 & 11,989 & 11,879 & 12,004 & 12,167 & 12,093 & 11,996 & 11,829 & 11,864 & 11,952 & 11,778 & 11,655 & 12,455 & 12,486 & 12,622 & 12,575 & 12,681 & 11,979 & 11,945 \\
\hline Ky. & Martin & 12,447 & 12,480 & 12,513 & 12,415 & 12,380 & 12,421 & 12,393 & 12,418 & 12,484 & 12,440 & 12,317 & 12,329 & 12,356 & 12,305 & 12,247 & 12,200 & 12,045 & 12,060 & 12,005 & 12,102 & 12,101 & 12,111 & 12,311 \\
\hline Ky. & Morgan & 11,568 & 11,006 & 11,974 & 11,821 & 11,893 & 12,228 & 11,091 & 11,563 & 0 & 0 & 0 & 10,859 & 0 & 0 & 0 & 11,628 & 11,327 & 11,153 & 11,216 & 0 & 0 & 0 & 0 \\
\hline Ky. & Owsley & 11,696 & 11,920 & 11,915 & 11,723 & 11,667 & 11,739 & 11,698 & 11,728 & 11,993 & 12,058 & 11,692 & 12,188 & 0 & 0 & 0 & 12,718 & 12,693 & 0 & 0 & 12,472 & 12,326 & 11,211 & 12,099 \\
\hline Ky. & Perry & 12,161 & 12,163 & 12,114 & 12,177 & 12,165 & 12,172 & 12,126 & 12,190 & 12,164 & 12,215 & 12,314 & 12,262 & 12,299 & 12,253 & 12,218 & 12,328 & 12,403 & 12,362 & 12,298 & 12,313 & 12,347 & 12,357 & 12,343 \\
\hline Ky. & Pike & 12,719 & 12,617 & 12,652 & 12,734 & 12,755 & 12,752 & 12,693 & 12,836 & 12,826 & 12,708 & 12,692 & 12,700 & 12,708 & 12,694 & 12,606 & 12,602 & 12,645 & 12,660 & 12,561 & 12,599 & 12,590 & 12,443 & 12,482 \\
\hline Ky. & Pulaski & 12,413 & 12,067 & 11,911 & 12,021 & 12,156 & 12,108 & 11,916 & 11,929 & 12,177 & 12,198 & 12,122 & 12,163 & 12,194 & 0 & 0 & 12,503 & 12,534 & 11,863 & 11,948 & 11,968 & 12,140 & 12,009 & 0 \\
\hline Ky. & Rockcastle & 11,609 & 0 & 11,124 & 0 & 0 & 0 & 0 & 0 & 0 & 0 & 11,201 & 0 & 0 & 0 & 0 & 0 & 0 & 0 & 0 & 0 & 0 & 0 & 11,782 \\
\hline Ку. & Wayne & 0 & 0 & 0 & 11,693 & 0 & 0 & 0 & 0 & 0 & 0 & 0 & 0 & 0 & nd & nd & nd & nd & nd & nd & nd & nd & nd & nd \\
\hline Ky. & Whitley & 12,747 & 12,725 & 12,662 & 12,828 & 12,920 & 12,817 & 12,660 & 12,692 & 12,747 & 12,753 & 12,700 & 12,674 & 12,832 & 12,741 & 12,704 & 12,566 & 12,562 & 12,505 & 12,565 & 12,456 & 12,505 & 12,634 & 12,589 \\
\hline Ky. & Wolfe & 11,666 & 12,120 & 11,911 & 11,791 & 11,755 & 11,420 & 12,483 & 0 & 12,071 & 12,260 & 12,251 & 12,370 & 12,195 & 11,735 & 11,232 & 11,502 & 11,347 & 0 & 0 & 0 & 0 & 0 & 0 \\
\hline Md. & Allegany & 11,984 & 11,735 & 12,127 & 11,975 & 11,950 & 12,153 & 11,792 & 11,721 & 11,825 & 11,921 & 12,231 & 12,156 & 12,059 & 12,036 & 12,026 & 11,855 & 11,974 & 12,239 & 12,315 & 11,638 & 11,575 & 11,504 & 11,456 \\
\hline Md. & Garrett & 12,515 & 12,404 & 12,550 & 12,652 & 12,605 & 12,788 & 12,723 & 12,822 & 12,749 & 12,636 & 12,652 & 12,820 & 12,859 & 12,655 & 12,476 & 12,367 & 12,309 & 12,162 & 12,018 & 12,311 & 12,440 & 12,446 & 12,182 \\
\hline Ohio & Athens & 10,719 & 0 & 0 & 0 & 11,727 & 11,638 & 11,508 & 11,418 & 11,385 & 0 & 0 & 0 & 0 & 0 & 0 & 0 & 0 & 0 & 0 & 10,701 & 0 & 11,425 & 10,791 \\
\hline Ohio & Belmont & 11,674 & 11,720 & 11,815 & 11,855 & 11,890 & 11,914 & 11,862 & 12,115 & 12,334 & 12,442 & 12,329 & 12,429 & 12,509 & 12,400 & 12,434 & 12,380 & 12,408 & 12,422 & 12,337 & 12,316 & 12,416 & 12,371 & 12,231 \\
\hline Ohio & Carroll & 11,786 & 11,827 & 11,871 & 11,751 & 11,923 & 11,880 & 11,777 & 11,619 & 12,172 & 12,094 & 12,172 & 12,117 & 12,096 & 0 & 11,921 & 0 & 12,152 & 0 & 0 & 12,389 & 12,365 & 12,372 & 12,674 \\
\hline Ohio & Columbiana & 12,185 & 11,981 & 12,108 & 12,092 & 12,326 & 12,267 & 12,168 & 12,119 & 12,157 & 12,306 & 12,086 & 12,138 & 12,399 & 12,415 & 12,408 & 12,357 & 12,268 & 12,364 & 12,329 & 12,050 & 12,130 & 12,124 & 12,321 \\
\hline Ohio & Coshocton & 12,073 & 11,946 & 12,012 & 12,131 & 12,085 & 11,917 & 11,935 & 11,888 & 11,998 & 12,166 & 12,022 & 11,859 & 11,953 & 11,893 & 11,787 & 11,939 & 11,983 & 12,018 & 11,692 & 11,714 & 11,737 & 11,678 & 11,730 \\
\hline Ohio & Gallia & 11,934 & 11,517 & 11,589 & 11,797 & 11,709 & 0 & 0 & 0 & 0 & 0 & 11,165 & 11,174 & 11,110 & 11,077 & 11,120 & 10,982 & 11,067 & 10,975 & 10,936 & 12,163 & 12,270 & 12,352 & 12,438 \\
\hline Ohio & Guernsey & 0 & 11,025 & 0 & 0 & 0 & 11,499 & 11,292 & 11,232 & 12,102 & 11,893 & 11,853 & 11,544 & 11,725 & 11,594 & 11,470 & 11,414 & 11,001 & 11,688 & 11,635 & 0 & 0 & 0 & 0 \\
\hline Ohio & Harrison & 12,378 & 12,283 & 12,332 & 12,493 & 12,481 & 12,377 & 12,387 & 12,303 & 12,238 & 12,250 & 12,468 & 12,181 & 12,262 & 12,339 & 12,302 & 12,327 & 12,184 & 12,289 & 12,124 & 12,332 & 12,098 & 12,079 & 12,125 \\
\hline Ohio & Hocking & 11,174 & 11,078 & 11,158 & 11,168 & 11,118 & 11,212 & 11,147 & 11,199 & 11,310 & 11,399 & 11,506 & 11,170 & 0 & nd & nd & nd & nd & nd & nd & nd & nd & nd & nd \\
\hline Ohio & Holmes & 0 & 0 & 0 & 0 & 0 & 11,347 & 11,439 & 11,329 & 11,648 & 11,875 & 11,707 & 11,559 & 11,639 & 11,557 & 11,527 & 11,318 & 11,535 & 11,598 & 11,718 & 11,559 & 0 & 0 & 0 \\
\hline
\end{tabular}


Table 2. Heating values of bituminous coal (in British thermal units per pound) that was mined in the Appalachian basin for electrical power generation.- Continued [Data are shown by county. Data for 1983 through 1995 are from Attanasi and Milici (1998); data for 1996 through 2005 are from the COALdat database. nd, no data]

\begin{tabular}{|c|c|c|c|c|c|c|c|c|c|c|c|c|c|c|c|c|c|c|c|c|c|c|c|c|}
\hline State & County & 1983 & 1984 & 1985 & 1986 & 1987 & 1988 & 1989 & 1990 & 1991 & 1992 & 1993 & 1994 & 1995 & 1996 & 1997 & 1998 & 1999 & 2000 & 2001 & 2002 & 2003 & 2004 & 2005 \\
\hline Ohio & Jackson & 11,302 & 11,161 & 11,228 & 11,401 & 11,266 & 11,131 & 11,205 & 11,141 & 11,153 & 11,163 & 11,162 & 11,176 & 11,063 & 10,985 & 11,032 & 10,937 & 10,922 & 11,180 & 11,325 & 11,361 & 11,421 & 11,482 & 11,222 \\
\hline Ohio & Jefferson & 2,050 & 12,013 & 11,642 & 11,690 & 11,895 & 11,964 & 11,950 & 11,938 & 12,101 & 12,124 & 12,086 & 11,934 & 12,081 & 11,879 & 11,565 & 11,885 & 11,904 & 11,795 & 11,800 & 12,011 & 11,940 & 11,875 & 11,940 \\
\hline Ohio & Lawrence & 11,338 & 11,315 & 11,213 & 11,320 & 11,276 & 11,106 & 11,176 & 11,368 & 11,446 & 11,522 & 11,669 & 11,687 & 11,761 & nd & nd & nd & nd & nd & nd & nd & nd & nd & nd \\
\hline Ohio & Mahoning & 11,816 & 11,950 & 11,887 & 11,901 & 12,151 & 12,052 & 12,234 & 12,188 & 11,948 & 12,222 & 12,357 & 12,046 & 11,923 & 0 & 11,560 & 0 & 0 & 0 & 0 & 0 & 0 & 0 & 0 \\
\hline Ohio & Meigs & 11,133 & 11,127 & 11,213 & 11,252 & 11,234 & 11,216 & 11,146 & 11,203 & 11,259 & 11,337 & 11,458 & 11,543 & 11,454 & 11,363 & 11,349 & 11,187 & 11,221 & 11,304 & 11,301 & 11,364 & 0 & 0 & 0 \\
\hline Ohio & onroe & 12,322 & 12,134 & 12,073 & 12,126 & 12,099 & 12,056 & 12,084 & 12,066 & 12,180 & 12,147 & 12,199 & 12,060 & 12,105 & 12,136 & 12,239 & 12,203 & 12,200 & 12,223 & 12,579 & 12,442 & 12,451 & 12,431 & 12,477 \\
\hline Ohio & Morgan & 11,386 & 11,390 & 11,452 & 0 & 0 & 0 & 11,454 & 0 & 0 & 0 & 0 & 0 & 0 & nd & nd & nd & nd & nd & nd & nd & nd & nd & nd \\
\hline Ohio & Muskingum & 11,283 & 11,367 & 11,437 & 11,463 & 11,560 & 11,555 & 11,521 & 11,661 & 11,724 & 11,559 & 11,437 & 11,517 & 11,678 & 11,601 & 11,476 & 11,387 & 11,534 & 11,409 & 11,507 & 11,247 & 0 & 0 & 11,457 \\
\hline Ohio & Noble & 11,404 & 11,391 & 11,455 & 11,446 & 11,451 & 11,470 & 11,329 & 11,415 & 11,452 & 11,425 & 11,438 & 11,505 & 11,676 & 11,582 & 11,598 & 11,548 & 11,559 & 11,640 & 11,644 & 11,662 & 11,648 & 11,406 & 11,277 \\
\hline Ohio & Perry & 11,151 & 11,263 & 11,303 & 11,277 & 11,346 & 11,445 & 11,403 & 11,548 & 11,550 & 11,275 & 11,146 & 11,307 & 11,282 & 11,312 & 11,305 & 11,280 & 11,246 & 11,499 & 11,489 & 11,154 & 11,685 & 11,660 & 12,569 \\
\hline Ohio & & 0 & 0 & 0 & 0 & 0 & 0 & 0 & 0 & 0 & 0 & 0 & 0 & 11,941 & nd & nd & nd & nd & nd & nd & nd & nd & nd & nd \\
\hline Ohio & cioto & 0 & 11,582 & 0 & 0 & 0 & 0 & 0 & 0 & 0 & 0 & 0 & 0 & 0 & 0 & 0 & & & 0 & 0 & 0 & 0 & 12,532 & 0 \\
\hline Ohio & Stark & 12,001 & 11,591 & 11,723 & 11,653 & 11,835 & 11,560 & 11,740 & 11,827 & 11,849 & 11,643 & 11,612 & 0 & 0 & 11,751 & 11,515 & 11,702 & 11,965 & 12,556 & 12,654 & 0 & 0 & 0 & 0 \\
\hline Ohio & & 11,741 & 11,997 & & & & & & & & & & & 11,495 & & & & & & 931 & 11,235 & 11,470 & 11,117 & 0 \\
\hline Ohio & Vinton & 11,511 & 10,992 & 11,031 & 11,177 & 11,270 & 11,470 & 11,188 & 11,259 & 11,373 & 11,284 & 11,355 & 11,300 & 11,138 & 11,451 & 11,416 & 11,381 & 11,341 & 11,391 & 11,351 & 11,341 & 11,316 & 11,503 & 11,553 \\
\hline Ohio & Washington & 0 & 0 & 0 & 0 & 0 & 0 & 0 & 11,713 & 0 & 0 & 0 & 0 & c & c & c & ( & ( & 0 & 0 & 0 & 12,983 & 13,018 & 13,044 \\
\hline $\mathrm{Pa}$. & Allegheny & 12,852 & 12,889 & 12,795 & 12,606 & 12,432 & 12,404 & 12,421 & 12,190 & 12,356 & 12,578 & 12,276 & 12,213 & 0 & 13,171 & 8,202 & 0 & 11,922 & 12,139 & 12,120 & 12,591 & 0 & 0 & 10,859 \\
\hline $\mathrm{Pa}$. & Armstrong & 12,445 & 12,488 & 12,466 & 12,412 & 12,327 & 12,419 & 12,419 & 12,308 & 12,397 & 12,369 & 12,289 & 12,240 & 12,293 & 12,321 & 12,260 & 12,189 & 12,334 & 12,380 & 12,374 & 12,252 & 12,351 & 12,412 & 12,616 \\
\hline $\mathrm{Pa}$. & Beaver & 0 & 0 & 0 & 12,086 & 11,984 & 12,002 & 12,096 & 12,148 & 12,228 & 12,174 & 11,919 & 11,790 & 0 & 0 & 0 & 0 & 12,172 & 12,144 & 0 & 12,080 & 12,235 & 11,860 & 12,128 \\
\hline $\mathrm{Pa}$. & Bedford & 12,555 & 12,655 & 12,901 & 12,730 & 12,671 & 13,072 & 12,576 & 12,641 & 12,538 & 9,490 & 10,632 & 0 & 0 & 0 & 0 & 0 & 12,758 & 12,360 & 0 & 0 & 7,788 & 8,500 & 0 \\
\hline $\mathrm{Pa}$. & & 0 & 0 & 0 & 11,921 & 0 & 0 & 0 & 0 & 0 & 13,124 & 13,121 & 0 & 0 & 11,117 & 11,062 & 0 & 0 & 0 & 0 & 0 & 0 & 0 & 0 \\
\hline $\mathrm{Pa}$. & Bradford & 10,462 & 0 & 0 & 0 & 0 & 0 & 0 & 0 & 0 & 0 & 0 & 0 & 0 & nd & nd & nd & nd & nd & nd & nd & nd & nd & nd \\
\hline $\mathrm{Pa}$. & Butler & 12,355 & 12,280 & 12,285 & 12,395 & 12,475 & 12,470 & 12,701 & 12,698 & 12,095 & 12,093 & 12,092 & 12,062 & 12,105 & 11,883 & 11,773 & 12,033 & 11,858 & 12,034 & 12,000 & 12,090 & 11,899 & 11,879 & 11,430 \\
\hline $\mathrm{Pa}$. & Cambria & 12,429 & 12,515 & 12,517 & 12,479 & 12,495 & 12,353 & 12,394 & 12,346 & 12,387 & 12,447 & 12,451 & 12,614 & 12,580 & 12,645 & 12,443 & 12,353 & 12,423 & 12,501 & 12,400 & 7,806 & 6,978 & 6,590 & 6,013 \\
\hline $\mathrm{Pa}$. & Cameron & 0 & 0 & 0 & 0 & 11,599 & 0 & 0 & 0 & 0 & 0 & 0 & 0 & 0 & nd & nd & nd & nd & nd & nd & nd & nd & nd & nd \\
\hline $\mathrm{Pa}$. & & 12,724 & 12,688 & 12,801 & 12,738 & 12,548 & 12,209 & 11,214 & 12,250 & 11,850 & 11,905 & 12,223 & 11,914 & 12,333 & 12,672 & 12,939 & 12,757 & 0 & 0 & 0 & 0 & 12,134 & 0 & 0 \\
\hline $\mathrm{Pa}$. & Clarion & 12,727 & 12,661 & 12,668 & 12,822 & 12,713 & 12,811 & 12,722 & 12,669 & 12,614 & 12,656 & 12,687 & 12,755 & 12,766 & 12,513 & 12,350 & 12,562 & 12,638 & 12,805 & 12,831 & 12,229 & 12,389 & 12,422 & 11,103 \\
\hline $\mathrm{Pa}$. & Clearfield & 12,514 & 12,453 & 12,492 & 12,491 & 12,488 & 12,492 & 12,395 & 12,339 & 12,374 & 12,398 & 12,409 & 12,446 & 12,492 & 12,352 & 12,323 & 12,364 & 12,447 & 12,357 & 12,302 & 11,717 & 11,777 & 11,320 & 11,411 \\
\hline $\mathrm{Pa}$. & Clinton & 12,930 & 0 & 11,579 & 10,537 & 11,924 & 12,246 & 10,772 & 10,974 & 11,171 & 10,736 & 0 & 11,850 & 0 & nd & nd & nd & nd & nd & nd & nd & nd & nd & nd \\
\hline $\mathrm{Pa}$. & Elk & 12,762 & 12,683 & 12,655 & 12,526 & 12,267 & 12,045 & 12,168 & 12,150 & 12,291 & 12,449 & 12,223 & 11,810 & 11,808 & 12,064 & 12,522 & 12,066 & 12,472 & 12,547 & 12,514 & 12,244 & 11,113 & 10,322 & 9,659 \\
\hline
\end{tabular}


Table 2. Heating values of bituminous coal (in British thermal units per pound) that was mined in the Appalachian basin for electrical power generation.-Continued

[Data are shown by county. Data for 1983 through 1995 are from Attanasi and Milici (1998); data for 1996 through 2005 are from the COALdat database. nd, no data]

\begin{tabular}{|c|c|c|c|c|c|c|c|c|c|c|c|c|c|c|c|c|c|c|c|c|c|c|c|c|}
\hline State & County & 1983 & 1984 & 1985 & 1986 & 1987 & 1988 & 1989 & 1990 & 1991 & 1992 & 1993 & 1994 & 1995 & 1996 & 1997 & 1998 & 1999 & 2000 & 2001 & 2002 & 2003 & 2004 & 2005 \\
\hline $\mathrm{Pa}$. & Fayette & 12,335 & 12,248 & 12,363 & 12,390 & 12,472 & 12,567 & 12,517 & 12,520 & 12,267 & 12,366 & 12,387 & 12,381 & 12,268 & 12,276 & 12,101 & 12,175 & 12,624 & 12,542 & 12,591 & 11,987 & 11,904 & 11,625 & 11,747 \\
\hline $\mathrm{Pa}$. & ulton & 624 & 461 &, 593 & ,944 & 597 & 871 &, 701 & 2,594 & 2,502 & 12,556 & 12,135 & 0 & 12,306 & 12,502 & 0 & 0 & 0 & 0 & 0 & 0 & 0 & 0 & 0 \\
\hline $\mathrm{Pa}$. & Greene & 2,164 & ,983 & 2,311 & 2,788 & 2,945 & 13,002 & 13,185 & 3,183 & 13,061 & 13,098 & 13,040 & 13,058 & 13,077 & 13,014 & 13,027 & 13,039 & 13,077 & 13,041 & 12,984 & 12,967 & 12,950 & 12,902 & 12,958 \\
\hline $\mathrm{Pa}$. & Huntingdon & 0 & 0 & 0 & 0 & 0 & 0 & 0 & 11,246 & 11,786 & 10,972 & 10,691 & 11,138 & 0 & nd & nd & nd & nd & nd & nd & nd & nd & nd & nd \\
\hline $\mathrm{Pa}$. & Indiana & 12,036 & 12,054 & 12,083 & 12,145 & 12,152 & 12,061 & 12,083 & 12,056 & 12,122 & 12,141 & 12,220 & 12,111 & 12,077 & 11,907 & 11,931 & 11,909 & 11,720 & 11,541 & 11,562 & 11,820 & 11,494 & 11,285 & 11,287 \\
\hline Pa. & & 12,768 & 12,809 & 12,767 & 12,794 & 12,707 & 12,625 & 12,594 & 12,537 & 12,704 & 12,601 & 12,467 & 12,693 & 12,608 & 12,462 & 12,390 & 12,492 & 12,534 & & 12,645 & 12,210 & 12,545 & 11,818 & 11,774 \\
\hline $\mathrm{Pa}$. & Lawrence & 11,986 & 801 & 12,021 & 11,971 & 12,167 & 11,930 & 11,759 & 11,972 & 12,021 & 12,142 & 12,278 & 0 & 12,041 & 12,093 & 0 & 0 & 0 & 11,239 & 0 & 0 & 8,065 & 0 & 9,735 \\
\hline $\mathrm{Pa}$. & Lycoming & 12,362 & 1,987 & 11,997 & 11,577 & 11,385 & 11,535 & 10,967 & 10,780 & 11,034 & 11,125 & 11,345 & 10,994 & 11,441 & 11,633 & 11,489 & 10,575 & 10,561 & 10,500 & 0 & 10,514 & 11,329 & 10,690 & 10,905 \\
\hline $\mathrm{Pa}$. & Mercer & 12,477 & 2,494 & 12,414 & 12,585 & 12,412 & 12,542 & 12,568 & 12,381 & 12,731 & 12,924 & 12,669 & 12,092 & 0 & 0 & 0 & 0 & 0 & 0 & 0 & 0 & 10,996 & 10,732 & 0 \\
\hline $\mathrm{Pa}$. & Somerset & 12,250 & 2,247 & 12,280 & 12,308 & 12,469 & 12,444 & 12,427 & 12,437 & 12,572 & 12,538 & 12,405 & 12,482 & 12,338 & 12,474 & 12,363 & 12,235 & 12,512 & 12,522 & 12,424 & 12,127 & 11,846 & 11,667 & 11,561 \\
\hline Pa. & & 435 & , 155 & ,205 & , 158 &, 304 & 1,153 & ,276 & 8,781 & 0 & 0 & 0 & 0 & 0 & 0 & 0 & 0 & 0 & ( & 0 & & 7,932 & 6,812 & 7,381 \\
\hline $\mathrm{Pa}$. & enango & 12,712 & 12,656 & 12,928 & 13,023 & 13,031 & 12,710 & 12,765 & 12,787 & 12,746 & 12,627 & 0 & 0 & 0 & 11,719 & 10,580 & 10,419 & 11,014 & c & 0 & 9,983 & 10,122 & 10,187 & 10,719 \\
\hline $\mathrm{Pa}$. & Washington & 12,193 & 12,752 & 12,775 & 12,921 & 12,937 & 12,973 & 12,540 & 12,542 & 12,636 & 12,839 & 12,474 & 12,475 & 12,847 & 13,148 & 13,144 & 13,160 & 13,161 & 13,127 & 13,114 & 13,066 & 12,980 & 12,962 & 12,983 \\
\hline $\mathrm{Pa}$. & Westmo & 12,173 &, 110 &, 206 &, 196 & 2,311 & 12,335 & 12,266 & 12,385 & 12,393 & 12,375 & 12,209 & 12,131 & 12,154 & 12,307 & 12,198 & 12,391 & 12,442 & 12,291 & 12,400 & 0 & 0 & 8,909 & 10,064 \\
\hline Tenn. & Anderson & 12,315 & 12,395 & 12,273 & 12,609 & 12,719 & 12,545 & 12,515 & 12,525 & 12,445 & 12,448 & 12,611 & 12,519 & 12,932 & 12,190 & 12,432 & 12,200 & 12,341 & 0 & 0 & 0 & 12,396 & 12,791 & 12,665 \\
\hline Tenn. & & 0 & 11,875 & 12,539 & 12,683 & 11,801 & 0 & 0 & 0 & 0 & 0 & 0 & 0 & 0 & nd & nd & nd & nd & nd & nd & nd & nd & nd & nd \\
\hline Tenn. & Campbell & 12,270 & 12,419 & 12,366 & 12,465 & 12,395 & 12,482 & 12,341 & 12,538 & 12,731 & 12,713 & 12,645 & 12,507 & 12,793 & 12,749 & 12,844 & 0 & 0 & c & 12,790 & 12,566 & 12,508 & 12,439 & 12,580 \\
\hline Tenn. & laiborne & 12,966 &, 950 & 12,711 & 12,546 & 12,677 & 12,717 & 12,827 & 12,919 & 12,583 & 12,703 & 0 & 0 & 12,567 & 12,603 & 12,706 & 12,855 & 12,851 & 13,085 & 12,789 & 13,126 & 13,030 & 12,806 & 12,894 \\
\hline Tenn. & Cumberland & 11,860 & 11,909 & 12,395 & 12,264 & 12,326 & 12,755 & 0 & 0 & 0 & 0 & 0 & 0 & 0 & 0 & 0 & 12,737 & 12,563 & 12,500 & 12,672 & 12,603 & 12,353 & 12,318 & 12,500 \\
\hline Tenn. & Fentress & 12,192 & 11,649 & 11,929 & 12,054 & 12,142 & 11,726 & 11,803 & 11,857 & 0 & 0 & 0 & 12,910 & 0 & 0 & 0 & 0 & 0 & 0 & 0 & 0 & 0 & 11,495 & 0 \\
\hline Tenn. & irundy & 12,409 & 12,355 & 12,491 & 12,443 & 12,150 & 12,598 & 12,266 & 11,930 & 11,871 & 0 & 0 & 0 & 0 & 0 & 0 & 0 & 0 & 0 & 0 & 0 & 0 & 0 & 12,858 \\
\hline Tenn. & Marion & 0 & 0 & 12,437 & 12,525 & 12,285 & 12,345 & 12,374 & 12,209 & 0 & 0 & 0 & 0 & 0 & nd & nd & nd & nd & nd & nd & nd & nd & nd & nd \\
\hline Tenn. & Morgan & 12,392 & 12,573 & 12,353 & 12,440 & 12,324 & 12,363 & 12,587 & 13,333 & 13,308 & 12,803 & 12,975 & 13,041 & 12,632 & 12,637 & 13,000 & 13,000 & 12,799 & 0 & 0 & 0 & 0 & 0 & 0 \\
\hline Tenn. & & 13,120 & 935 &, 217 & & 12,944 & 0 & 0 & 0 & 0 & 0 & 0 & 0 & 0 & nd & nd & nd & nd & nd & nd & nd & nd & nd & nd \\
\hline Tenn. & Scott & 12,032 & 12,107 & 12,219 & 12,397 & 12,507 & 13,001 & 13,024 & 13,039 & 13,324 & 13,626 & 13,377 & 13,238 & 13,131 & 12,732 & 12,944 & 12,748 & 12,326 & 12,604 & 12,710 & 0 & 0 & 12,094 & 12,491 \\
\hline Tenn. & Sequatchie & 11,679 & 12,066 & 11,965 & 12,269 & 12,581 & 12,480 & 12,285 & 12,366 & 12,322 & 12,442 & 12,354 & 12,421 & 12,483 & 12,423 & 12,386 & 12,382 & 12,400 & 12,553 & 0 & 0 & 0 & 0 & 0 \\
\hline Tenn. & Van Buren & 11,411 & 0 & 0 & 0 & 0 & 0 & 0 & 0 & 0 & 0 & 0 & 0 & 0 & nd & nd & nd & nd & nd & nd & nd & nd & nd & nd \\
\hline Va. & Buchanan & 12,825 & 12,908 & 12,975 & 13,078 & 13,143 & 13,247 & 13,260 & 13,373 & 13,440 & 13,489 & 13,427 & 13,302 & 13,448 & 13,247 & 13,445 & 13,551 & 13,403 & 13,432 & 13,393 & 13,527 & 13,230 & 13,106 & 12,318 \\
\hline Va. & Dickenson & 12,907 & 13,071 & 13,141 & 13,176 & 13,127 & 13,288 & 13,046 & 13,224 & 12,951 & 12,842 & 12,870 & 12,736 & 12,863 & 12,601 & 12,779 & 12,800 & 12,914 & 12,907 & 12,871 & 13,183 & 12,907 & 12,750 & 12,861 \\
\hline Va. & Lee & 12,960 & 12,950 & 12,944 & 12,927 & 12,662 & 12,541 & 12,518 & 12,587 & 12,637 & 12,546 & 12,642 & 12,616 & 12,417 & 12,540 & 12,674 & 12,643 & 12,638 & 12,735 & 12,505 & 12,502 & 12,359 & 12,333 & 12,539 \\
\hline
\end{tabular}


Table 2. Heating values of bituminous coal (in British thermal units per pound) that was mined in the Appalachian basin for electrical power generation.- Continued [Data are shown by county. Data for 1983 through 1995 are from Attanasi and Milici (1998); data for 1996 through 2005 are from the COALdat database. nd, no data]

\begin{tabular}{|c|c|c|c|c|c|c|c|c|c|c|c|c|c|c|c|c|c|c|c|c|c|c|c|c|}
\hline State & County & 1983 & 1984 & 1985 & 1986 & 1987 & 1988 & 1989 & 1990 & 1991 & 1992 & 1993 & 1994 & 1995 & 1996 & 1997 & 1998 & 1999 & 2000 & 2001 & 2002 & 2003 & 2004 & 2005 \\
\hline Va. & Russell & 12,680 & 12,969 & 13,087 & 12,901 & 12,926 & 12,803 & 12,677 & 12,562 & 12,421 & 12,471 & 12,539 & 12,536 & 12,389 & 12,440 & 12,348 & 12,280 & 12,444 & 12,321 & 12,419 & 12,315 & 12,272 & 12,288 & 12,376 \\
\hline Va. & Scott & 0 & 0 & 0 & 12,869 & 0 & 0 & 0 & 0 & 0 & 0 & 0 & 0 & 0 & nd & nd & nd & nd & nd & nd & nd & nd & nd & nd \\
\hline Va. & Tazewell & 13,139 & 13,106 & 13,220 & 13,435 & 13,399 & 13,586 & 13,475 & 13,175 & 0 & 0 & 0 & 0 & 0 & 13,576 & 0 & 0 & 0 & 0 & 0 & 12,399 & 12,595 & 12,504 & 12,708 \\
\hline Va. & Wise & 12,634 & 12,661 & 12,718 & 12,823 & 12,934 & 12,990 & 12,875 & 12,944 & 12,855 & 12,827 & 12,795 & 12,744 & 12,801 & 12,778 & 12,793 & 12,800 & 12,831 & 12,904 & 12,788 & 12,897 & 12,831 & 12,665 & 12,629 \\
\hline W. Va. & Barbour & 12,769 & 12,585 & 12,531 & 12,480 & 12,645 & 12,999 & 12,848 & 12,880 & 12,848 & 12,821 & 12,805 & 12,945 & 12,995 & 12,889 & 12,792 & 12,818 & 12,954 & 12,936 & 13,061 & 12,719 & 12,644 & 12,968 & 12,966 \\
\hline W. Va. & oone & 12,407 & 12,552 & 12,666 & 12,623 & 12,743 & 12,707 & 12,529 & 12,507 & 12,521 & 12,445 & 12,473 & 12,422 & 12,435 & 12,376 & 12,288 & 12,300 & 12,369 & 12,294 & 12,176 & 12,325 & 12,271 & 12,254 & 12,278 \\
\hline W. Va. & Braxton & 13,133 & 3,330 & 3,165 & 13,546 & 13,514 & 13,351 & 12,958 & 12,949 & 13,071 & 0 & 12,366 & 0 & 0 & nd & nd & nd & nd & nd & nd & nd & nd & nd & nd \\
\hline W. Va. & rooke & 11,932 & 11,760 & 11,980 & 12,190 & 12,204 & 12,169 & 12,207 & 12,244 & 12,213 & 12,124 & 12,240 & 12,203 & 12,304 & 12,410 & 12,362 & 12,422 & 12,307 & 12,308 & 12,257 & 12,322 & 0 & 0 & 0 \\
\hline W. Va. & Clay & 0 & 13,190 & 0 & 11,633 & 12,097 & 12,156 & 12,063 & 12,175 & 12,202 & 12,161 & 12,109 & 12,163 & 12,198 & 12,358 & 12,410 & 12,415 & 12,415 & 12,436 & 12,288 & 12,404 & 12,460 & 12,383 & 12,361 \\
\hline W. Va. & Fayette & 12,263 & 11,949 & 12,490 & 12,558 & 12,240 & 12,238 & 12,245 & 12,439 & 12,403 & 12,413 & 12,434 & 12,486 & 12,545 & 12,557 & 12,442 & 12,288 & 12,479 & 12,672 & 12,585 & 12,285 & 12,035 & 12,402 & 12,577 \\
\hline W. Va. & Th & 2,864 & 2,581 & 0 & 0 & 12,984 & 2,924 & 0 & 0 & 0 & 0 & 0 & 0 & 0 & nd & nd & nd & nd & nd & nd & nd & nd & nd & nd \\
\hline W. Va. & Grant & 12,109 & 12,181 & 12,144 & 12,357 & 12,505 & 12,509 & 12,468 & 12,592 & 12,601 & 12,622 & 12,614 & 12,420 & 12,432 & 12,564 & 12,551 & 12,146 & 12,336 & 12,201 & 12,033 & 12,068 & 11,827 & 11,972 & 11,741 \\
\hline W. Va. & Greenbrier & 13,346 & 13,094 & 13,246 & 13,200 & 12,921 & 12,009 & 11,654 & 0 & 13,026 & 12,607 & 12,696 & 12,610 & 11,636 & 0 & 0 & 0 & 13,059 & 13,078 & 12,943 & 12,894 & 13,211 & 0 & 0 \\
\hline W. Va. & arrison & 13,087 & 3,005 & 13,025 & 12,992 & 13,076 & 13,124 & 13,062 & 13,039 & 13,111 & 13,091 & 13,066 & 13,053 & 12,490 & 12,549 & 12,632 & 12,656 & 12,584 & 12,519 & 12,339 & 12,374 & 12,382 & 12,328 & 12,305 \\
\hline W. Va. & Kanawha & 11,718 & 11,669 & 11,754 & 11,877 & 11,857 & 11,880 & 11,777 & 11,968 & 11,973 & 12,005 & 12,234 & 12,316 & 12,366 & 12,142 & 12,053 & 11,989 & 12,113 & 12,099 & 12,131 & 12,292 & 12,303 & 12,234 & 12,148 \\
\hline W. Va. & ewis & 12,864 & 12,793 & 12,896 & 12,951 & 12,898 & 12,900 & 12,837 & 12,968 & 0 & 0 & 12,950 & 12,463 & 12,577 & nd & nd & nd & nd & nd & nd & nd & nd & nd & nd \\
\hline W. Va. & Lincoln & 0 & 0 & 0 & 0 & 12,722 & 12,499 & 12,030 & 11,948 & 11,712 & 11,916 & 11,895 & 11,792 & 12,161 & 12,139 & 11,995 & 11,756 & 11,738 & 12,971 & 12,651 & 12,632 & 12,718 & 12,573 & 12,354 \\
\hline W. Va. & Logan & 12,614 & 12,540 & 12,495 & 12,438 & 12,457 & 12,407 & 12,351 & 12,410 & 12,437 & 12,369 & 12,304 & 12,344 & 12,281 & 12,206 & 12,121 & 12,092 & 12,230 & 12,161 & 12,068 & 12,385 & 12,311 & 12,179 & 12,121 \\
\hline W. Va. & McDowell & 13,253 & 13,048 & 12,600 & 12,847 & 13,133 & 12,870 & 12,775 & 12,995 & 12,921 & 13,742 & 13,339 & 13,147 & 11,842 & 12,290 & 0 & 0 & 13,756 & 13,456 & 12,946 & 13,262 & 13,442 & 13,521 & 13,632 \\
\hline W. Va. & Marion & 12,530 & 12,561 & 12,531 & 12,466 & 12,340 & 12,302 & 12,383 & 12,415 & 12,332 & 12,293 & 12,829 & 12,341 & 12,363 & 13,085 & 13,173 & 13,125 & 12,882 & 0 & 12,998 & 12,403 & 10,458 & 12,133 & 12,562 \\
\hline W. Va. & Marshall & 12,150 & 12,173 & 12,174 & 12,163 & 12,118 & 12,083 & 12,160 & 12,124 & 12,199 & 12,186 & 12,271 & 12,111 & 12,149 & 12,089 & 12,120 & 12,137 & 12,165 & 12,156 & 12,120 & 12,326 & 12,397 & 12,334 & 12,287 \\
\hline W. Va. & Mason & 11,546 & 0 & 0 & 0 & 11,648 & 11,648 & 11,844 & 12,072 & 12,077 & 11,788 & 11,508 & 11,250 & 0 & 0 & 0 & 0 & 0 & 13,596 & 0 & 0 & 0 & 12,399 & 0 \\
\hline W. Va. & Mercer & 0 & 0 & 13,106 & 12,202 & 12,803 & 0 & 0 & 0 & 0 & 0 & 0 & 0 & 0 & 0 & 0 & 0 & 0 & 0 & 0 & 13,018 & 0 & 0 & 0 \\
\hline W. Va. & Mineral & 11,862 & 11,608 & 11,736 & 11,676 & 12,144 & 12,278 & 12,048 & 11,778 & 11,694 & 11,709 & 11,692 & 12,035 & 11,811 & 11,714 & 0 & 0 & 0 & 11,951 & 0 & 0 & 0 & 0 & 11,975 \\
\hline W. Va. & Mingo & 12,570 & 12,441 & 12,347 & 12,440 & 12,451 & 12,398 & 12,403 & 12,486 & 12,578 & 12,570 & 12,595 & 12,615 & 12,533 & 12,511 & 12,452 & 12,430 & 12,340 & 12,325 & 12,267 & 12,172 & 12,195 & 12,150 & 12,259 \\
\hline W. Va. & Monongalia & 12,930 & 12,704 & 12,714 & 12,778 & 12,728 & 12,800 & 12,738 & 12,876 & 12,954 & 13,002 & 12,883 & 12,881 & 12,924 & 12,885 & 12,899 & 12,877 & 12,927 & 12,899 & 12,966 & 12,759 & 12,538 & 12,563 & 12,671 \\
\hline W. Va. & Nicholas & 12,868 & 12,874 & 12,852 & 12,880 & 12,796 & 12,808 & 12,768 & 12,679 & 12,764 & 12,727 & 12,668 & 12,554 & 12,603 & 12,537 & 12,783 & 12,372 & 12,378 & 12,359 & 12,120 & 12,493 & 12,392 & 12,149 & 12,026 \\
\hline W. Va. & Ohio & 13,302 & 0 & 10,721 & 10,748 & 10,935 & 11,696 & 12,432 & 11,855 & 11,845 & 12,197 & 12,181 & 12,187 & 12,202 & 0 & 0 & 0 & 0 & 12,043 & 0 & 0 & 0 & 0 & 0 \\
\hline W. Va. & Preston & 12,464 & 12,378 & 12,585 & 12,620 & 12,592 & 12,562 & 12,528 & 12,570 & 12,688 & 12,610 & 12,573 & 12,707 & 12,907 & 12,911 & 12,950 & 12,928 & 13,058 & 13,140 & 13,217 & 13,136 & 12,927 & 12,915 & 13,053 \\
\hline W. Va. & Raleigh & 12,421 & 13,676 & 13,808 & 13,574 & 0 & 0 & 13,113 & 13,581 & 13,325 & 13,449 & 13,830 & 0 & 0 & 11,879 & 11,332 & 11,374 & 11,434 & 10,904 & 11,615 & 12,950 & 12,768 & 12,340 & 12,472 \\
\hline
\end{tabular}


Table 2. Heating values of bituminous coal (in British thermal units per pound) that was mined in the Appalachian basin for electrical power generation.-Continued

[Data are shown by county. Data for 1983 through 1995 are from Attanasi and Milici (1998); data for 1996 through 2005 are from the COALdat database. nd, no data]

\begin{tabular}{|c|c|c|c|c|c|c|c|c|c|c|c|c|c|c|c|c|c|c|c|c|c|c|c|c|}
\hline State & County & 1983 & 1984 & 1985 & 1986 & 1987 & 1988 & 1989 & 1990 & 1991 & 1992 & 1993 & 1994 & 1995 & 1996 & 1997 & 1998 & 1999 & 2000 & 2001 & 2002 & 2003 & 2004 & 2005 \\
\hline W. Va. & Randolph & 13,104 & 13,019 & 13,154 & 13,189 & 12,990 & 12,749 & 12,568 & 12,124 & 11,979 & 12,701 & 12,109 & 12,714 & 11,973 & 0 & 0 & 0 & 0 & 0 & 0 & 0 & 12,697 & 0 & 0 \\
\hline W. Va. & Taylor & 0 & 0 & 12,177 & 12,148 & 0 & 12,117 & 11,971 & 11,970 & 0 & 12,234 & 11,893 & 0 & 0 & nd & nd & nd & nd & nd & nd & nd & nd & nd & nd \\
\hline W. Va. & Tucker & 11,855 & 11,558 & 11,555 & 11,636 & 0 & 0 & 0 & 0 & 0 & 0 & 0 & 0 & 0 & nd & nd & nd & nd & nd & nd & nd & nd & nd & nd \\
\hline W. Va. & Upshur & 13,069 & 13,025 & 13,022 & 13,107 & 13,088 & 13,144 & 12,927 & 12,938 & 13,061 & 13,095 & 12,838 & 12,868 & 12,679 & 12,551 & 12,510 & 12,758 & 12,956 & 12,892 & 12,925 & 12,836 & 12,649 & 12,121 & 12,307 \\
\hline W. Va. & Wayne & 12,458 & 0 & 11,290 & 12,083 & 11,305 & 11,646 & 11,947 & 12,149 & 12,290 & 12,030 & 11,873 & 11,876 & 11,911 & 11,940 & 11,985 & 11,968 & 11,934 & 11,942 & 11,946 & 12,135 & 12,307 & 12,193 & 12,036 \\
\hline W. Va. & Webster & 13,005 & 12,995 & 13,054 & 13,011 & 12,881 & 12,999 & 12,963 & 13,148 & 13,120 & 13,010 & 12,815 & 12,914 & 12,754 & 12,692 & 12,632 & 12,616 & 12,498 & 12,543 & 12,498 & 12,438 & 12,421 & 12,372 & 12,400 \\
\hline W. Va. & Wyoming & 13,737 & 13,856 & 13,767 & 13,679 & 13,469 & 13,270 & 13,725 & 0 & 0 & 0 & 13,793 & 13,089 & 12,833 & 12,119 & 12,720 & 13,000 & 13,708 & 13,649 & 12,869 & 12,885 & 12,819 & 12,876 & 12,837 \\
\hline
\end{tabular}


Table 3. Weighted average sulfur content (in weight percent) of bituminous coal that was mined in the Appalachian basin for electrical power generation. [Data are shown by county. Data for 1983 through 1995 are from Attanasi and Milici (1998); data for 1996 through 2005 are from the COALdat database. nd, no data]

\begin{tabular}{|c|c|c|c|c|c|c|c|c|c|c|c|c|c|c|c|}
\hline State & County & 1983 & 1984 & 1985 & 1986 & 1987 & 1988 & 1989 & 1990 & 1991 & 1992 & 1993 & 1994 & 1995 & $\begin{array}{l}\text { Weighted } \\
\text { average } \\
(1983-1995)\end{array}$ \\
\hline Ala. & Bibb & 0.00 & 0.00 & 0.00 & 0.00 & 0.00 & 0.00 & 0.00 & 0.00 & 0.00 & 0.00 & 0.00 & 0.00 & 0.73 & 0.73 \\
\hline Ala. & Blount & 0.00 & 2.37 & 2.27 & 1.80 & 1.09 & 1.00 & 0.00 & 2.45 & 1.96 & 0.00 & 0.00 & 0.00 & 0.00 & 2.21 \\
\hline Ala. & Cullman & 0.72 & 0.72 & 0.00 & 1.55 & 0.00 & 0.00 & 0.00 & 0.00 & 0.00 & 0.00 & 0.00 & 0.00 & 0.00 & 0.74 \\
\hline Ala. & De Kalb & 0.00 & 0.00 & 0.00 & 1.18 & 0.00 & 0.00 & 0.00 & 0.00 & 0.00 & 0.00 & 0.00 & 0.00 & 0.00 & 1.18 \\
\hline Ala. & Fayette & 2.32 & 2.39 & 2.22 & 2.06 & 2.07 & 2.20 & 2.05 & 2.13 & 2.26 & 2.10 & 1.86 & 1.82 & 1.81 & 2.08 \\
\hline Ala. & Jackson & 1.22 & 1.20 & 1.23 & 0.52 & 0.60 & 0.00 & 0.00 & 0.00 & 0.00 & 0.00 & 0.00 & 1.73 & 0.00 & 1.27 \\
\hline Ala. & Jefferson & 1.24 & 1.45 & 1.35 & 1.33 & 1.20 & 1.25 & 1.39 & 1.31 & 1.11 & 1.23 & 1.15 & 1.08 & 1.03 & 1.23 \\
\hline Ala. & Lamar & 0.00 & 0.00 & 0.00 & 0.00 & 0.00 & 1.38 & 1.36 & 0.00 & 0.00 & 0.00 & 0.00 & 0.00 & 0.00 & 1.37 \\
\hline Ala. & Marion & 0.00 & 1.56 & 1.14 & 1.12 & 1.07 & 1.19 & 1.33 & 1.42 & 1.83 & 1.57 & 1.48 & 1.26 & 0.00 & 1.30 \\
\hline Ala. & Marshall & 1.99 & 1.86 & 0.00 & 0.00 & 0.00 & 0.00 & 0.00 & 0.00 & 0.00 & 0.00 & 0.00 & 0.00 & 0.00 & 1.94 \\
\hline Ala. & St. Clair & 0.00 & 0.00 & 1.34 & 0.00 & 1.18 & 1.42 & 0.00 & 0.00 & 0.00 & 0.00 & 0.00 & 0.00 & 0.00 & 1.31 \\
\hline Ala. & Shelby & 0.00 & 0.00 & 0.00 & 0.00 & 0.00 & 0.00 & 0.00 & 0.00 & 0.00 & 0.00 & 0.00 & 0.00 & 0.84 & 0.84 \\
\hline Ala. & Tuscaloosa & 1.84 & 1.97 & 1.46 & 1.51 & 1.40 & 1.43 & 1.36 & 1.16 & 1.03 & 1.09 & 0.98 & 0.98 & 1.07 & 1.19 \\
\hline Ala. & Walker & 1.28 & 1.21 & 1.16 & 1.21 & 1.11 & 1.11 & 1.10 & 1.14 & 1.19 & 1.10 & 1.04 & 1.05 & 1.00 & 1.14 \\
\hline Ala. & Winston & 0.00 & 0.00 & 1.37 & 0.94 & 1.39 & 0.97 & 1.45 & 1.49 & 1.35 & 1.35 & 1.16 & 1.23 & 0.00 & 1.31 \\
\hline $\mathrm{Ga}$. & Dade & 1.50 & 1.61 & 0.00 & 0.00 & 0.00 & 0.00 & 0.00 & 0.00 & 0.00 & 0.00 & 0.00 & 0.00 & 0.00 & 1.60 \\
\hline Ky. & Bell & 1.32 & 1.30 & 1.29 & 1.24 & 1.28 & 1.29 & 1.23 & 1.33 & 1.26 & 1.19 & 1.26 & 1.31 & 1.37 & 1.28 \\
\hline Ky. & Boyd & 1.19 & 1.26 & 2.44 & 1.74 & 0.95 & 0.61 & 0.91 & 0.69 & 0.72 & 0.92 & 0.86 & 0.84 & 0.76 & 1.15 \\
\hline Ку. & Breathitt & 0.88 & 0.84 & 0.85 & 0.89 & 1.04 & 1.06 & 1.02 & 1.09 & 1.17 & 1.08 & 1.16 & 1.09 & 0.96 & 0.99 \\
\hline Ку. & Carter & 1.93 & 1.09 & 0.96 & 0.98 & 1.06 & 1.03 & 0.78 & 1.25 & 1.49 & 1.39 & 0.00 & 1.52 & 0.00 & 1.14 \\
\hline Ку. & Clay & 1.55 & 1.62 & 1.54 & 1.57 & 1.59 & 1.58 & 1.40 & 1.37 & 1.42 & 1.09 & 1.44 & 1.36 & 1.16 & 1.54 \\
\hline Ку. & Clinton & 0.00 & 0.00 & 0.84 & 0.00 & 0.00 & 0.00 & 0.00 & 0.00 & 0.00 & 0.00 & 0.00 & 0.00 & 0.00 & 0.84 \\
\hline Ку. & Elliott & 1.53 & 1.67 & 1.68 & 1.49 & 1.63 & 0.00 & 1.34 & 1.18 & 1.14 & 0.00 & 0.00 & 0.00 & 0.00 & 1.57 \\
\hline Ky. & Estill & 3.52 & 0.00 & 0.00 & 0.73 & 0.00 & 0.00 & 0.00 & 0.00 & 0.00 & 0.00 & 0.75 & 1.41 & 0.95 & 0.88 \\
\hline Ky. & Floyd & 1.06 & 1.07 & 1.10 & 1.08 & 1.10 & 1.06 & 1.03 & 0.97 & 0.96 & 0.94 & 1.08 & 1.07 & 0.95 & 1.04 \\
\hline Ky. & Greenup & 1.66 & 1.60 & 2.76 & 2.93 & 2.18 & 2.36 & 1.83 & 2.44 & 2.56 & 2.40 & 2.19 & 1.98 & 0.80 & 2.11 \\
\hline Ky. & Harlan & 1.20 & 1.14 & 1.03 & 1.04 & 1.06 & 1.08 & 1.18 & 1.18 & 1.13 & 1.09 & 1.14 & 1.06 & 1.03 & 1.10 \\
\hline Ky. & Jackson & 1.03 & 1.37 & 1.49 & 1.94 & 0.00 & 0.95 & 0.98 & 1.45 & 1.65 & 2.19 & 0.00 & 0.92 & 0.88 & 1.29 \\
\hline Ky. & Johnson & 1.20 & 1.19 & 1.02 & 1.15 & 1.17 & 1.25 & 1.32 & 1.21 & 1.19 & 1.25 & 1.29 & 1.38 & 1.23 & 1.21 \\
\hline Ky. & Knott & 1.17 & 1.04 & 1.09 & 1.14 & 0.90 & 0.78 & 1.20 & 1.21 & 1.13 & 1.05 & 1.14 & 1.05 & 0.97 & 1.07 \\
\hline
\end{tabular}


Table 3. Weighted average sulfur content (in weight percent) of bituminous coal that was mined in the Appalachian basin for electrical power generation.-Continued [Data are shown by county. Data for 1983 through 1995 are from Attanasi and Milici (1998); data for 1996 through 2005 are from the COALdat database. nd, no data]

\begin{tabular}{|c|c|c|c|c|c|c|c|c|c|c|c|c|}
\hline State & County & 1996 & 1997 & 1998 & 1999 & 2000 & 2001 & 2002 & 2003 & 2004 & 2005 & $\begin{array}{l}\text { Weighted } \\
\text { average } \\
(1996-2005)\end{array}$ \\
\hline Ala. & Bibb & 1.80 & 0.92 & 1.01 & 0.00 & 0.00 & 0.00 & 0.00 & 0.00 & 1.73 & 1.79 & 1.50 \\
\hline Ala. & Blount & nd & nd & nd & nd & nd & nd & nd & nd & nd & nd & No production \\
\hline Ala. & Cullman & nd & nd & nd & nd & nd & nd & nd & nd & nd & nd & No production \\
\hline Ala. & De Kalb & nd & nd & nd & nd & nd & nd & nd & nd & nd & nd & No production \\
\hline Ala. & Fayette & 1.83 & 0.00 & 0.00 & 0.00 & 0.00 & 1.59 & 1.76 & 0.00 & 0.00 & 0.00 & 1.65 \\
\hline Ala. & Jackson & 0.00 & 0.00 & 0.00 & 0.00 & 0.88 & 0.00 & 0.00 & 0.86 & 0.86 & 0.67 & 0.77 \\
\hline Ala. & Jefferson & 0.92 & 0.76 & 0.72 & 0.78 & 0.73 & 0.79 & 0.75 & 0.75 & 1.06 & 1.27 & 0.84 \\
\hline Ala. & Lamar & nd & nd & nd & nd & nd & nd & nd & nd & nd & nd & No production \\
\hline Ala. & Marion & nd & nd & nd & nd & nd & nd & nd & nd & nd & nd & No production \\
\hline Ala. & Marshall & nd & nd & nd & nd & nd & nd & nd & nd & nd & nd & No production \\
\hline Ala. & St. Clair & nd & nd & nd & nd & nd & nd & nd & nd & nd & nd & No production \\
\hline Ala. & Shelby & 0.70 & 0.68 & 0.73 & 0.00 & 0.71 & 0.67 & 0.00 & 0.00 & 0.00 & 0.00 & 0.70 \\
\hline Ala. & Tuscaloosa & 1.46 & 1.25 & 1.20 & 1.17 & 1.21 & 1.21 & 1.51 & 1.47 & 1.66 & 1.75 & 1.35 \\
\hline Ala. & Walker & 1.27 & 1.66 & 1.67 & 1.35 & 1.55 & 1.39 & 1.61 & 1.52 & 1.58 & 1.68 & 1.53 \\
\hline Ala. & Winston & nd & nd & nd & nd & nd & nd & nd & nd & nd & nd & No production \\
\hline Ga. & Dade & nd & nd & nd & nd & nd & nd & nd & nd & nd & nd & No production \\
\hline Ку. & Bell & 1.33 & 1.42 & 1.43 & 1.34 & 1.26 & 1.39 & 1.06 & 1.07 & 0.97 & 1.05 & 1.29 \\
\hline Ку. & Boyd & 0.72 & 0.72 & 0.85 & 0.77 & 0.87 & 0.83 & 1.05 & 1.00 & 0.98 & 0.96 & 0.90 \\
\hline Ky. & Breathitt & 1.09 & 1.50 & 1.44 & 1.28 & 1.03 & 1.15 & 1.61 & 1.49 & 1.25 & 1.54 & 1.35 \\
\hline Ky. & Carter & 0.00 & 0.00 & 0.86 & 0.00 & 0.00 & 0.00 & 0.00 & 0.00 & 0.00 & 0.00 & 0.86 \\
\hline Ку. & Clay & 1.18 & 1.14 & 1.10 & 1.13 & 1.40 & 1.27 & 1.22 & 1.27 & 1.66 & 1.40 & 1.26 \\
\hline Ку. & Clinton & nd & nd & nd & nd & nd & nd & nd & nd & nd & nd & No production \\
\hline Ку. & Elliott & nd & nd & nd & nd & nd & nd & nd & nd & nd & nd & No production \\
\hline Ку. & Estill & 0.88 & 0.82 & 0.00 & 0.91 & 1.21 & 1.06 & 0.66 & 0.67 & 0.70 & 0.00 & 1.03 \\
\hline Ку. & Floyd & 1.00 & 1.09 & 0.93 & 0.95 & 0.92 & 1.05 & 1.18 & 1.13 & 1.33 & 1.28 & 1.05 \\
\hline Ку. & Greenup & 0.81 & 0.79 & 0.91 & 0.89 & 0.88 & 0.84 & 0.00 & 0.00 & 0.00 & 0.00 & 0.85 \\
\hline Ку. & Harlan & 1.11 & 1.08 & 1.05 & 1.04 & 1.04 & 1.07 & 1.06 & 1.03 & 1.03 & 1.00 & 1.05 \\
\hline Ky. & Jackson & 0.00 & 0.00 & 0.00 & 0.00 & 0.00 & 0.00 & 0.83 & 0.00 & 0.00 & 0.00 & 0.83 \\
\hline Ку. & Johnson & 1.28 & 1.30 & 1.30 & 1.30 & 1.13 & 1.10 & 1.07 & 1.02 & 1.05 & 1.03 & 1.19 \\
\hline Ку. & Knott & 1.11 & 1.14 & 1.13 & 1.12 & 1.03 & 1.08 & 1.08 & 1.14 & 1.20 & 1.14 & 1.12 \\
\hline
\end{tabular}


Table 3. Weighted average sulfur content (in weight percent) of bituminous coal that was mined in the Appalachian basin for electrical power generation.-Continued [Data are shown by county. Data for 1983 through 1995 are from Attanasi and Milici (1998); data for 1996 through 2005 are from the COALdat database. nd, no data]

\begin{tabular}{|c|c|c|c|c|c|c|c|c|c|c|c|c|c|c|c|}
\hline State & County & 1983 & 1984 & 1985 & 1986 & 1987 & 1988 & 1989 & 1990 & 1991 & 1992 & 1993 & 1994 & 1995 & $\begin{array}{l}\text { Weighted } \\
\text { average } \\
\text { (1983-1995) }\end{array}$ \\
\hline Ky. & Knox & 1.13 & 1.16 & 1.37 & 1.52 & 1.56 & 1.42 & 1.24 & 1.05 & 0.75 & 0.99 & 1.17 & 1.83 & 1.09 & 1.36 \\
\hline Ky. & Laurel & 1.53 & 1.71 & 1.51 & 1.54 & 1.45 & 1.28 & 1.17 & 1.11 & 0.85 & 1.41 & 1.97 & 0.00 & 1.63 & 1.48 \\
\hline Ky. & Lawrence & 1.42 & 1.53 & 0.85 & 1.32 & 1.06 & 1.10 & 1.10 & 1.35 & 1.12 & 1.07 & 1.06 & 1.21 & 1.43 & 1.19 \\
\hline Ky. & Lee & 0.00 & 0.00 & 1.97 & 2.04 & 1.02 & 1.05 & 1.47 & 2.20 & 0.00 & 0.00 & 1.69 & 0.00 & 1.15 & 1.84 \\
\hline Ky. & Leslie & 1.42 & 1.43 & 1.32 & 1.25 & 1.31 & 1.51 & 1.38 & 1.35 & 1.26 & 1.23 & 1.35 & 1.19 & 1.13 & 1.30 \\
\hline Kу. & Letcher & 0.88 & 0.84 & 0.90 & 0.82 & 0.78 & 0.80 & 0.84 & 0.85 & 0.91 & 0.95 & 1.05 & 1.09 & 1.11 & 0.92 \\
\hline Ky. & McCreary & 1.22 & 1.06 & 1.48 & 1.53 & 1.63 & 2.29 & 0.00 & 0.00 & 0.00 & 0.99 & 1.11 & 0.96 & 0.92 & 1.49 \\
\hline Ky. & Magoffin & 0.98 & 1.04 & 1.09 & 1.08 & 1.10 & 1.09 & 1.11 & 1.08 & 1.14 & 1.11 & 1.01 & 0.99 & 1.05 & 1.08 \\
\hline Ky. & Martin & 0.86 & 0.89 & 0.93 & 0.92 & 0.92 & 0.91 & 0.91 & 0.94 & 0.88 & 0.88 & 0.94 & 0.94 & 0.83 & 0.90 \\
\hline Ky. & Morgan & 1.30 & 2.06 & 1.27 & 1.29 & 0.84 & 1.36 & 1.36 & 1.34 & 0.00 & 0.00 & 0.00 & 1.43 & 0.00 & 1.14 \\
\hline Ky. & Owsley & 1.21 & 1.00 & 1.03 & 1.78 & 2.00 & 2.25 & 1.90 & 1.24 & 1.44 & 1.25 & 1.88 & 1.52 & 0.00 & 1.71 \\
\hline Ky. & Perry & 1.13 & 1.12 & 1.08 & 1.12 & 1.10 & 1.12 & 1.09 & 1.05 & 1.07 & 1.00 & 1.13 & 1.09 & 0.99 & 1.08 \\
\hline Ky. & Pike & 0.85 & 0.94 & 0.91 & 0.94 & 0.90 & 0.88 & 0.92 & 0.94 & 0.94 & 0.96 & 1.00 & 0.98 & 0.89 & 0.93 \\
\hline Ky. & Pulaski & 1.63 & 1.66 & 1.78 & 1.96 & 1.92 & 1.65 & 1.46 & 1.56 & 1.58 & 1.65 & 1.72 & 1.60 & 1.41 & 1.67 \\
\hline Ку. & Rockcastle & 1.27 & 0.00 & 0.99 & 0.00 & 0.00 & 0.00 & 0.00 & 0.00 & 0.00 & 0.00 & 0.50 & 0.00 & 0.00 & 1.21 \\
\hline Ky. & Wayne & 0.00 & 0.00 & 0.00 & 0.59 & 0.00 & 0.00 & 0.00 & 0.00 & 0.00 & 0.00 & 0.00 & 0.00 & 0.00 & 0.59 \\
\hline Ky. & Whitley & 1.31 & 1.23 & 1.19 & 1.09 & 1.09 & 1.12 & 1.16 & 1.27 & 1.20 & 1.31 & 1.23 & 1.25 & 1.02 & 1.18 \\
\hline Ку. & Wolfe & 1.19 & 2.42 & 2.64 & 2.61 & 2.86 & 2.38 & 1.55 & 0.00 & 1.18 & 1.23 & 1.28 & 1.30 & 1.27 & 2.21 \\
\hline Md. & Allegany & 1.79 & 1.81 & 1.39 & 1.54 & 1.62 & 1.63 & 1.78 & 1.72 & 1.65 & 1.61 & 1.59 & 1.45 & 1.54 & 1.66 \\
\hline Md. & Garrett & 1.67 & 1.66 & 1.68 & 1.56 & 1.63 & 1.58 & 1.60 & 1.59 & 1.57 & 1.69 & 1.68 & 1.63 & 1.51 & 1.61 \\
\hline Ohio & Athens & 2.78 & 0.00 & 0.00 & 0.00 & 1.72 & 2.69 & 2.72 & 2.27 & 2.43 & 0.00 & 0.00 & 0.00 & 0.00 & 2.33 \\
\hline Ohio & Belmont & 2.93 & 2.92 & 2.85 & 2.88 & 2.89 & 2.90 & 3.14 & 3.50 & 3.71 & 3.74 & 3.72 & 3.99 & 3.96 & 3.26 \\
\hline Ohio & Carroll & 1.94 & 2.39 & 2.94 & 3.08 & 2.65 & 2.93 & 2.43 & 2.12 & 2.78 & 2.48 & 2.59 & 2.78 & 3.00 & 2.66 \\
\hline Ohio & Columbiana & 2.25 & 2.48 & 2.57 & 2.37 & 2.32 & 2.58 & 2.70 & 2.49 & 2.70 & 2.17 & 2.19 & 2.23 & 2.25 & 2.43 \\
\hline Ohio & Coshocton & 3.18 & 3.21 & 3.21 & 3.07 & 3.23 & 3.22 & 3.22 & 3.23 & 3.21 & 3.26 & 3.20 & 2.92 & 2.52 & 3.13 \\
\hline Ohio & Gallia & 3.53 & 3.10 & 3.07 & 3.55 & 3.16 & 0.00 & 0.00 & 0.00 & 0.00 & 0.00 & 3.37 & 3.07 & 2.97 & 3.20 \\
\hline Ohio & Guernsey & 0.00 & 4.56 & 0.00 & 0.00 & 0.00 & 2.65 & 4.13 & 4.19 & 1.91 & 1.87 & 2.14 & 2.68 & 3.04 & 3.33 \\
\hline Ohio & Harrison & 2.63 & 2.70 & 2.63 & 2.51 & 2.41 & 2.49 & 2.55 & 2.51 & 3.16 & 3.16 & 2.75 & 3.33 & 3.89 & 2.86 \\
\hline Ohio & Hocking & 3.32 & 3.50 & 3.31 & 3.30 & 3.15 & 3.24 & 3.47 & 3.55 & 3.56 & 3.50 & 3.58 & 3.68 & 0.00 & 3.39 \\
\hline Ohio & Holmes & 0.00 & 0.00 & 0.00 & 0.00 & 0.00 & 3.41 & 3.69 & 3.72 & 3.44 & 3.43 & 3.56 & 3.65 & 3.37 & 3.56 \\
\hline
\end{tabular}


Table 3. Weighted average sulfur content (in weight percent) of bituminous coal that was mined in the Appalachian basin for electrical power generation.-Continued [Data are shown by county. Data for 1983 through 1995 are from Attanasi and Milici (1998); data for 1996 through 2005 are from the COALdat database. nd, no data]

\begin{tabular}{|c|c|c|c|c|c|c|c|c|c|c|c|c|}
\hline State & County & 1996 & 1997 & 1998 & 1999 & 2000 & 2001 & 2002 & 2003 & 2004 & 2005 & $\begin{array}{l}\text { Weighted } \\
\text { average } \\
(1996-2005)\end{array}$ \\
\hline Ky. & Knox & 0.00 & 0.00 & 3.53 & 0.00 & 0.00 & 0.00 & 0.94 & 1.09 & 1.68 & 1.68 & 1.68 \\
\hline Ky. & Laurel & 0.87 & 0.00 & 1.23 & 1.54 & 0.80 & 0.66 & 0.00 & 1.06 & 0.85 & 0.00 & 0.96 \\
\hline Ку. & Lawrence & 0.94 & 0.84 & 0.91 & 1.15 & 1.24 & 1.18 & 1.04 & 1.38 & 1.41 & 1.18 & 1.25 \\
\hline Ky. & Lee & 0.00 & 0.00 & 0.00 & 0.00 & 0.00 & 0.00 & 0.91 & 0.00 & 0.00 & 0.00 & 0.91 \\
\hline Ky. & Leslie & 1.08 & 1.02 & 1.03 & 1.10 & 0.98 & 1.09 & 1.23 & 1.28 & 1.23 & 1.16 & 1.07 \\
\hline Ky. & Letcher & 1.16 & 1.21 & 1.22 & 1.41 & 1.12 & 1.19 & 1.10 & 1.19 & 1.19 & 1.25 & 1.21 \\
\hline Ку. & McCreary & 0.93 & 0.00 & 0.00 & 0.00 & 0.00 & 0.00 & 0.00 & 0.00 & 0.00 & 0.00 & 0.93 \\
\hline Ky. & Magoffin & 0.94 & 1.66 & 0.98 & 0.80 & 0.68 & 0.70 & 0.70 & 0.72 & 0.86 & 0.97 & 0.95 \\
\hline Ку. & Martin & 0.88 & 0.91 & 0.93 & 0.86 & 0.90 & 0.89 & 0.84 & 0.86 & 0.93 & 1.02 & 0.90 \\
\hline Ку. & Morgan & 0.00 & 0.00 & 0.67 & 0.64 & 0.81 & 1.17 & 0.00 & 0.00 & 0.00 & 0.00 & 1.00 \\
\hline Ку. & Owsley & 0.00 & 0.00 & 1.24 & 1.71 & 0.00 & 0.00 & 0.83 & 1.54 & 1.48 & 1.54 & 1.47 \\
\hline Ky. & Perry & 0.99 & 0.99 & 1.04 & 1.00 & 0.97 & 1.03 & 0.99 & 1.01 & 1.01 & 1.03 & 1.01 \\
\hline Ky. & Pike & 0.93 & 1.00 & 0.97 & 0.97 & 0.93 & 1.01 & 1.03 & 1.03 & 1.01 & 1.10 & 1.00 \\
\hline Ку. & Pulaski & 0.00 & 0.00 & 1.37 & 1.32 & 1.20 & 1.41 & 1.30 & 1.26 & 1.28 & 0.00 & 1.28 \\
\hline Ку. & Rockcastle & 0.00 & 0.00 & 0.00 & 0.00 & 0.00 & 0.00 & 0.00 & 0.00 & 0.00 & 0.78 & 0.78 \\
\hline Ky. & Wayne & nd & nd & nd & nd & nd & nd & nd & nd & nd & nd & No production \\
\hline Ку. & Whitley & 1.05 & 1.04 & 1.16 & 0.98 & 1.16 & 1.00 & 0.96 & 1.08 & 1.12 & 1.20 & 1.10 \\
\hline Ky. & Wolfe & 0.97 & 0.90 & 0.99 & 1.00 & 0.00 & 0.00 & 0.00 & 0.00 & 0.00 & 0.00 & 0.94 \\
\hline Md. & Allegany & 1.41 & 1.30 & 1.44 & 1.34 & 1.02 & 0.98 & 1.60 & 1.58 & 1.64 & 1.73 & 1.57 \\
\hline Md. & Garrett & 1.57 & 1.67 & 1.68 & 1.87 & 1.79 & 1.65 & 1.69 & 1.39 & 1.67 & 1.91 & 1.69 \\
\hline Ohio & Athens & 0.00 & 0.00 & 0.00 & 0.00 & 0.00 & 0.00 & 1.87 & 0.00 & 1.85 & 1.54 & 1.70 \\
\hline Ohio & Belmont & 3.88 & 3.80 & 3.80 & 3.88 & 3.80 & 3.89 & 3.89 & 4.00 & 4.02 & 4.04 & 3.90 \\
\hline Ohio & Carroll & 0.00 & 2.46 & 0.00 & 3.82 & 0.00 & 0.00 & 1.58 & 1.64 & 1.94 & 1.32 & 1.74 \\
\hline Ohio & Columbiana & 2.14 & 2.43 & 2.25 & 2.34 & 2.19 & 2.25 & 2.59 & 2.88 & 2.71 & 2.62 & 2.39 \\
\hline Ohio & Coshocton & 2.68 & 2.68 & 2.70 & 2.70 & 2.36 & 2.15 & 2.47 & 2.27 & 2.23 & 2.24 & 2.44 \\
\hline Ohio & Gallia & 2.83 & 2.84 & 2.69 & 2.81 & 2.84 & 2.70 & 3.65 & 3.80 & 3.70 & 3.61 & 3.60 \\
\hline Ohio & Guernsey & 4.89 & 4.31 & 4.40 & 2.04 & 3.06 & 3.19 & 0.00 & 0.00 & 0.00 & 0.00 & 4.53 \\
\hline Ohio & Harrison & 3.14 & 3.14 & 2.97 & 2.59 & 2.63 & 2.54 & 2.63 & 2.90 & 2.85 & 2.79 & 2.82 \\
\hline Ohio & Hocking & nd & nd & nd & nd & nd & nd & nd & nd & nd & nd & No production \\
\hline Ohio & Holmes & 3.38 & 3.44 & 3.49 & 3.58 & 3.81 & 3.89 & 4.21 & 0.00 & 0.00 & 0.00 & 3.62 \\
\hline
\end{tabular}


Table 3. Weighted average sulfur content (in weight percent) of bituminous coal that was mined in the Appalachian basin for electrical power generation.-Continued [Data are shown by county. Data for 1983 through 1995 are from Attanasi and Milici (1998); data for 1996 through 2005 are from the COALdat database. nd, no data]

\begin{tabular}{|c|c|c|c|c|c|c|c|c|c|c|c|c|c|c|c|}
\hline State & County & 1983 & 1984 & 1985 & 1986 & 1987 & 1988 & 1989 & 1990 & 1991 & 1992 & 1993 & 1994 & 1995 & $\begin{array}{l}\text { Weighted } \\
\text { average } \\
\text { (1983-1995) }\end{array}$ \\
\hline Ohio & Jackson & 2.34 & 2.95 & 3.03 & 2.33 & 2.94 & 3.15 & 3.17 & 3.29 & 3.25 & 3.49 & 3.46 & 3.39 & 3.70 & 3.22 \\
\hline Ohio & Jefferson & 2.83 & 3.08 & 2.93 & 2.86 & 2.66 & 2.70 & 2.87 & 3.00 & 2.99 & 3.08 & 3.12 & 3.23 & 2.73 & 2.92 \\
\hline Ohio & Lawrence & 3.46 & 3.30 & 3.01 & 3.25 & 2.44 & 2.91 & 2.41 & 2.53 & 2.74 & 2.57 & 2.57 & 2.84 & 2.82 & 2.71 \\
\hline Ohio & Mahoning & 2.98 & 2.74 & 2.82 & 3.11 & 3.06 & 3.57 & 3.41 & 2.96 & 3.12 & 4.01 & 3.25 & 3.62 & 3.02 & 3.15 \\
\hline Ohio & Meigs & 3.48 & 3.40 & 3.34 & 3.39 & 3.44 & 3.41 & 3.25 & 3.43 & 3.44 & 3.36 & 3.29 & 3.10 & 3.04 & 3.34 \\
\hline Ohio & Monroe & 3.85 & 4.09 & 4.14 & 4.15 & 4.04 & 3.96 & 4.01 & 4.14 & 4.10 & 3.87 & 3.32 & 3.72 & 3.65 & 4.00 \\
\hline Ohio & Morgan & 4.36 & 4.27 & 4.32 & 0.00 & 0.00 & 0.00 & 2.48 & 0.00 & 0.00 & 0.00 & 0.00 & 0.00 & 0.00 & 4.16 \\
\hline Ohio & Muskingum & 4.24 & 4.15 & 4.26 & 4.31 & 4.11 & 4.21 & 4.24 & 4.20 & 4.34 & 4.27 & 4.52 & 4.53 & 4.63 & 4.25 \\
\hline Ohio & Noble & 4.38 & 4.28 & 4.27 & 4.40 & 4.57 & 4.62 & 4.59 & 4.59 & 4.74 & 4.41 & 4.58 & 4.73 & 4.90 & 4.58 \\
\hline Ohio & Perry & 2.44 & 2.47 & 2.14 & 2.13 & 2.06 & 2.37 & 2.51 & 2.37 & 3.04 & 2.86 & 2.69 & 3.35 & 3.20 & 2.38 \\
\hline Ohio & Pike & 0.00 & 0.00 & 0.00 & 0.00 & 0.00 & 0.00 & 0.00 & 0.00 & 0.00 & 0.00 & 0.00 & 0.00 & 2.60 & 2.60 \\
\hline Ohio & Scioto & 0.00 & 1.08 & 0.00 & 0.00 & 0.00 & 0.00 & 0.00 & 0.00 & 0.00 & 0.00 & 0.00 & 0.00 & 0.00 & 1.08 \\
\hline Ohio & Stark & 3.07 & 4.10 & 3.57 & 3.66 & 3.18 & 3.29 & 3.89 & 3.33 & 3.97 & 2.66 & 2.62 & 0.00 & 0.00 & 3.49 \\
\hline Ohio & Tuscarawas & 3.62 & 3.14 & 3.25 & 2.88 & 3.03 & 3.18 & 3.09 & 3.02 & 3.35 & 3.21 & 3.39 & 3.41 & 3.50 & 3.24 \\
\hline Ohio & Vinton & 3.28 & 3.45 & 3.38 & 3.24 & 3.06 & 2.95 & 3.15 & 3.20 & 3.23 & 3.23 & 3.24 & 3.12 & 2.97 & 3.18 \\
\hline Ohio & Washington & 0.00 & 0.00 & 0.00 & 0.00 & 0.00 & 0.00 & 0.00 & 0.00 & 0.00 & 0.00 & 0.00 & 0.00 & 0.00 & 0.00 \\
\hline Pa. & Allegheny & 1.26 & 1.17 & 1.35 & 1.43 & 1.31 & 1.32 & 1.38 & 1.42 & 1.53 & 1.67 & 2.78 & 2.16 & 0.00 & 1.40 \\
\hline $\mathrm{Pa}$. & Armstrong & 1.82 & 1.66 & 1.58 & 1.57 & 1.53 & 1.66 & 1.69 & 1.71 & 1.74 & 1.71 & 1.89 & 1.79 & 1.79 & 1.70 \\
\hline $\mathrm{Pa}$. & Beaver & 0.00 & 0.00 & 0.00 & 3.28 & 2.97 & 2.62 & 1.95 & 1.99 & 2.85 & 2.15 & 1.72 & 1.59 & 0.00 & 2.40 \\
\hline Pa. & Bedford & 2.12 & 2.03 & 1.98 & 1.92 & 1.68 & 1.90 & 1.47 & 1.83 & 2.31 & 1.17 & 1.36 & 0.00 & 0.00 & 1.90 \\
\hline $\mathrm{Pa}$. & Blair & 0.00 & 0.00 & 0.00 & 2.12 & 0.00 & 0.00 & 0.00 & 0.00 & 0.00 & 1.83 & 1.64 & 0.00 & 0.00 & 1.81 \\
\hline $\mathrm{Pa}$. & Bradford & 0.82 & 0.00 & 0.00 & 0.00 & 0.00 & 0.00 & 0.00 & 0.00 & 0.00 & 0.00 & 0.00 & 0.00 & 0.00 & 0.82 \\
\hline Pa. & Butler & 1.83 & 1.86 & 1.87 & 2.17 & 2.44 & 2.63 & 2.07 & 1.88 & 1.84 & 2.19 & 1.80 & 1.92 & 1.72 & 2.08 \\
\hline $\mathrm{Pa}$. & Cambria & 1.54 & 1.58 & 1.47 & 1.49 & 1.45 & 1.72 & 1.66 & 1.72 & 1.71 & 1.91 & 1.87 & 1.87 & 1.81 & 1.64 \\
\hline $\mathrm{Pa}$. & Cameron & 0.00 & 0.00 & 0.00 & 0.00 & 0.58 & 0.00 & 0.00 & 0.00 & 0.00 & 0.00 & 0.00 & 0.00 & 0.00 & 0.58 \\
\hline $\mathrm{Pa}$. & Centre & 1.84 & 1.96 & 1.82 & 1.99 & 1.85 & 1.62 & 1.58 & 1.65 & 1.66 & 1.76 & 1.75 & 1.65 & 1.64 & 1.77 \\
\hline Pa. & Clarion & 2.39 & 2.44 & 2.34 & 2.27 & 2.00 & 2.17 & 2.22 & 2.25 & 2.21 & 2.27 & 2.05 & 1.77 & 1.61 & 2.21 \\
\hline Pa. & Clearfield & 1.82 & 1.87 & 1.82 & 1.81 & 1.86 & 1.85 & 1.82 & 1.95 & 1.91 & 1.93 & 1.87 & 1.85 & 1.79 & 1.86 \\
\hline Pa. & Clinton & 1.47 & 0.00 & 0.89 & 0.60 & 1.64 & 2.03 & 0.67 & 0.96 & 0.71 & 0.72 & 0.00 & 1.57 & 0.00 & 1.01 \\
\hline Pa. & Elk & 1.79 & 1.94 & 1.62 & 1.80 & 1.63 & 1.92 & 1.77 & 1.75 & 1.66 & 1.68 & 1.86 & 1.55 & 1.37 & 1.75 \\
\hline
\end{tabular}


Table 3. Weighted average sulfur content (in weight percent) of bituminous coal that was mined in the Appalachian basin for electrical power generation.-Continued [Data are shown by county. Data for 1983 through 1995 are from Attanasi and Milici (1998); data for 1996 through 2005 are from the COALdat database. nd, no data]

\begin{tabular}{|c|c|c|c|c|c|c|c|c|c|c|c|c|}
\hline State & County & 1996 & 1997 & 1998 & 1999 & 2000 & 2001 & 2002 & 2003 & 2004 & 2005 & $\begin{array}{l}\text { Weighted } \\
\text { average } \\
(1996-2005)\end{array}$ \\
\hline Ohio & Jackson & 3.65 & 3.42 & 3.55 & 3.52 & 3.11 & 2.98 & 3.17 & 3.36 & 3.42 & 3.70 & 3.40 \\
\hline Ohio & Jefferson & 3.59 & 3.00 & 2.46 & 2.29 & 2.46 & 2.50 & 2.37 & 2.37 & 2.21 & 2.34 & 2.48 \\
\hline Ohio & Lawrence & 0.00 & 2.84 & 0.00 & 0.00 & 0.00 & 0.00 & 0.00 & 0.00 & 0.00 & 0.00 & 2.84 \\
\hline Ohio & Mahoning & nd & nd & nd & nd & nd & nd & nd & nd & nd & nd & No production \\
\hline Ohio & Meigs & 3.20 & 3.52 & 3.52 & 3.53 & 3.54 & 3.62 & 3.68 & 0.00 & 0.00 & 0.00 & 3.48 \\
\hline Ohio & Monroe & 4.28 & 4.32 & 4.41 & 4.37 & 3.87 & 3.73 & 4.19 & 4.13 & 4.37 & 4.33 & 4.30 \\
\hline Ohio & Morgan & nd & nd & nd & nd & nd & nd & nd & nd & nd & nd & No production \\
\hline Ohio & Muskingum & 4.95 & 4.32 & 4.41 & 4.58 & 3.13 & 3.00 & 2.91 & 0.00 & 0.00 & 3.73 & 3.61 \\
\hline Ohio & Noble & 4.97 & 5.06 & 5.09 & 4.64 & 3.03 & 2.78 & 2.49 & 1.99 & 2.55 & 4.01 & 3.64 \\
\hline Ohio & Perry & 2.93 & 3.01 & 2.79 & 2.76 & 3.34 & 3.55 & 3.71 & 3.35 & 3.81 & 4.07 & 3.35 \\
\hline Ohio & Pike & nd & nd & nd & nd & nd & nd & nd & nd & nd & nd & No production \\
\hline Ohio & Scioto & 0.00 & 0.00 & 0.00 & 0.00 & 0.00 & 0.00 & 0.00 & 0.00 & 1.93 & 0.00 & 1.93 \\
\hline Ohio & Stark & 3.86 & 3.56 & 3.21 & 3.01 & 2.34 & 1.94 & 0.00 & 0.00 & 0.00 & 0.00 & 3.24 \\
\hline Ohio & Tuscarawas & 3.33 & 2.97 & 3.47 & 3.35 & 3.57 & 3.61 & 3.78 & 3.98 & 2.83 & 0.00 & 3.45 \\
\hline Ohio & Vinton & 2.71 & 3.00 & 2.96 & 3.00 & 2.92 & 3.17 & 3.22 & 2.99 & 3.21 & 3.02 & 2.99 \\
\hline Ohio & Washington & 0.00 & 0.00 & 0.00 & 0.00 & 0.00 & 0.00 & 0.00 & 2.59 & 2.61 & 2.70 & 2.63 \\
\hline $\mathrm{Pa}$. & Allegheny & 2.42 & 0.85 & 0.00 & 1.43 & 2.31 & 2.36 & 0.73 & 0.00 & 0.00 & 1.62 & 1.50 \\
\hline Pa. & Armstrong & 1.95 & 2.13 & 2.16 & 1.93 & 2.20 & 2.01 & 2.22 & 2.41 & 2.41 & 2.28 & 2.22 \\
\hline $\mathrm{Pa}$. & Beaver & 0.00 & 0.00 & 0.00 & 0.90 & 2.16 & 0.00 & 1.80 & 1.70 & 2.55 & 2.09 & 2.19 \\
\hline Pa. & Bedford & 0.00 & 0.00 & 0.00 & 1.12 & 0.96 & 0.00 & 0.00 & 0.92 & 0.97 & 0.00 & 0.95 \\
\hline $\mathrm{Pa}$. & Blair & 2.83 & 2.26 & 0.00 & 0.00 & 0.00 & 0.00 & 0.00 & 0.00 & 0.00 & 0.00 & 2.54 \\
\hline Pa. & Bradford & nd & nd & nd & nd & nd & nd & nd & nd & nd & nd & No production \\
\hline Pa. & Butler & 1.95 & 1.83 & 2.06 & 1.97 & 1.59 & 1.60 & 1.76 & 1.04 & 2.08 & 2.69 & 1.88 \\
\hline Pa. & Cambria & 1.82 & 2.03 & 2.15 & 2.21 & 2.04 & 2.50 & 2.06 & 2.30 & 2.25 & 2.39 & 2.21 \\
\hline Pa. & Cameron & nd & nd & nd & nd & nd & nd & nd & nd & nd & nd & No production \\
\hline Pa. & Centre & 1.46 & 1.25 & 1.17 & 0.00 & 0.00 & 0.00 & 0.00 & 2.20 & 0.00 & 0.00 & 1.52 \\
\hline Pa. & Clarion & 1.71 & 1.80 & 1.61 & 1.73 & 1.26 & 1.25 & 2.08 & 1.12 & 1.68 & 1.93 & 1.67 \\
\hline $\mathrm{Pa}$. & Clearfield & 1.89 & 1.90 & 1.80 & 1.81 & 1.74 & 1.80 & 1.70 & 1.68 & 1.78 & 1.67 & 1.80 \\
\hline Pa. & Clinton & nd & nd & nd & nd & nd & nd & nd & nd & nd & nd & No production \\
\hline Pa. & Elk & 1.29 & 1.74 & 1.94 & 1.61 & 1.91 & 2.24 & 1.89 & 1.60 & 1.79 & 1.63 & 1.74 \\
\hline
\end{tabular}


Table 3. Weighted average sulfur content (in weight percent) of bituminous coal that was mined in the Appalachian basin for electrical power generation.-Continued [Data are shown by county. Data for 1983 through 1995 are from Attanasi and Milici (1998); data for 1996 through 2005 are from the COALdat database. nd, no data]

\begin{tabular}{|c|c|c|c|c|c|c|c|c|c|c|c|c|c|c|c|}
\hline State & County & 1983 & 1984 & 1985 & 1986 & 1987 & 1988 & 1989 & 1990 & 1991 & 1992 & 1993 & 1994 & 1995 & $\begin{array}{l}\text { Weighted } \\
\text { average } \\
\text { (1983-1995) }\end{array}$ \\
\hline $\mathrm{Pa}$. & Fayette & 1.97 & 2.13 & 1.99 & 1.93 & 1.80 & 1.85 & 1.75 & 1.85 & 1.56 & 1.67 & 1.71 & 1.41 & 1.82 & 1.85 \\
\hline $\mathrm{Pa}$. & Fulton & 1.40 & 1.93 & 1.90 & 2.18 & 1.92 & 2.05 & 2.09 & 2.33 & 2.03 & 2.38 & 2.09 & 0.00 & 1.54 & 2.01 \\
\hline $\mathrm{Pa}$. & Greene & 1.82 & 1.92 & 1.70 & 1.62 & 1.60 & 1.59 & 1.58 & 1.76 & 1.78 & 1.79 & 1.97 & 1.82 & 1.70 & 1.77 \\
\hline Pa. & Huntingdon & 0.00 & 0.00 & 0.00 & 0.00 & 0.00 & 0.00 & 0.00 & 0.72 & 1.66 & 1.59 & 1.35 & 2.36 & 0.00 & 1.91 \\
\hline Pa. & Indiana & 1.92 & 1.93 & 1.99 & 2.03 & 1.95 & 1.92 & 1.86 & 1.93 & 1.95 & 1.87 & 1.78 & 1.87 & 1.70 & 1.92 \\
\hline Pa. & Jefferson & 1.77 & 1.72 & 1.72 & 1.71 & 1.81 & 1.83 & 1.71 & 1.63 & 1.66 & 1.71 & 1.77 & 1.57 & 1.55 & 1.71 \\
\hline Pa. & Lawrence & 1.99 & 2.62 & 2.60 & 2.50 & 1.85 & 2.11 & 2.32 & 2.45 & 2.03 & 2.74 & 2.58 & 0.00 & 1.42 & 2.26 \\
\hline $\mathrm{Pa}$. & Lycoming & 0.89 & 0.88 & 0.78 & 0.78 & 0.82 & 0.88 & 0.86 & 0.97 & 0.93 & 0.99 & 0.93 & 0.94 & 0.97 & 0.90 \\
\hline Pa. & Mercer & 2.29 & 1.51 & 1.50 & 1.60 & 1.33 & 1.54 & 1.56 & 1.62 & 1.69 & 1.98 & 1.52 & 2.85 & 0.00 & 1.72 \\
\hline Pa. & Somerset & 1.46 & 1.24 & 1.26 & 1.39 & 1.53 & 1.60 & 1.55 & 1.67 & 1.71 & 1.95 & 1.99 & 1.90 & 2.08 & 1.73 \\
\hline Pa. & Tioga & 1.16 & 1.14 & 1.02 & 0.86 & 1.01 & 1.34 & 1.28 & 0.83 & 0.00 & 0.00 & 0.00 & 0.00 & 0.00 & 1.10 \\
\hline Pa. & Venango & 3.37 & 3.30 & 3.14 & 2.52 & 2.48 & 2.31 & 2.19 & 2.34 & 2.05 & 1.64 & 0.00 & 0.00 & 0.00 & 2.64 \\
\hline Pa. & Washington & 1.75 & 1.76 & 1.88 & 1.77 & 1.54 & 1.70 & 1.99 & 2.29 & 2.52 & 2.26 & 2.57 & 2.45 & 1.90 & 2.11 \\
\hline Pa. & Westmoreland & 1.55 & 1.23 & 1.15 & 1.37 & 1.90 & 2.13 & 1.87 & 1.91 & 2.07 & 2.09 & 2.16 & 2.17 & 2.08 & 1.92 \\
\hline Tenn. & Anderson & 1.75 & 1.58 & 1.45 & 1.50 & 1.69 & 1.92 & 2.13 & 2.22 & 2.05 & 2.33 & 1.56 & 1.52 & 0.68 & 1.85 \\
\hline Tenn. & Bledsoe & 0.00 & 1.20 & 1.17 & 1.30 & 0.60 & 0.00 & 0.00 & 0.00 & 0.00 & 0.00 & 0.00 & 0.00 & 0.00 & 1.18 \\
\hline Tenn. & Campbell & 1.83 & 1.77 & 1.69 & 1.66 & 1.56 & 1.74 & 1.21 & 1.31 & 1.20 & 1.31 & 1.40 & 1.24 & 1.06 & 1.48 \\
\hline Tenn. & Claiborne & 0.88 & 0.90 & 0.97 & 1.01 & 1.03 & 1.03 & 1.06 & 1.13 & 1.52 & 1.12 & 0.00 & 0.00 & 1.45 & 1.01 \\
\hline Tenn. & Cumberland & 1.39 & 1.20 & 0.79 & 0.85 & 0.95 & 0.87 & 0.00 & 0.00 & 0.00 & 0.00 & 0.00 & 0.00 & 0.00 & 1.07 \\
\hline Tenn. & Fentress & 1.91 & 2.43 & 2.61 & 1.93 & 1.76 & 3.10 & 1.33 & 1.90 & 0.00 & 0.00 & 0.00 & 1.36 & 0.00 & 2.14 \\
\hline Tenn. & Grundy & 0.89 & 0.86 & 0.95 & 0.89 & 0.81 & 0.80 & 0.75 & 0.92 & 0.88 & 0.00 & 0.00 & 0.00 & 0.00 & 0.88 \\
\hline Tenn. & Marion & 0.00 & 0.00 & 1.70 & 0.84 & 1.48 & 0.89 & 0.71 & 0.70 & 0.00 & 0.00 & 0.00 & 0.00 & 0.00 & 0.81 \\
\hline Tenn. & Morgan & 1.17 & 0.91 & 1.45 & 1.17 & 1.37 & 1.34 & 1.38 & 2.08 & 1.82 & 1.82 & 2.27 & 2.40 & 1.32 & 1.48 \\
\hline Tenn. & Rhea & 1.20 & 1.88 & 1.34 & 1.55 & 1.20 & 0.00 & 0.00 & 0.00 & 0.00 & 0.00 & 0.00 & 0.00 & 0.00 & 1.60 \\
\hline Tenn. & Scott & 1.86 & 2.28 & 1.76 & 1.83 & 1.44 & 1.33 & 1.66 & 1.55 & 1.53 & 1.70 & 1.61 & 1.47 & 1.28 & 1.59 \\
\hline Tenn. & Sequatchie & 2.79 & 3.10 & 3.40 & 1.10 & 0.74 & 0.79 & 0.83 & 0.79 & 1.00 & 0.80 & 0.91 & 0.87 & 0.86 & 0.92 \\
\hline Tenn. & Van Buren & 1.80 & 0.00 & 0.00 & 0.00 & 0.00 & 0.00 & 0.00 & 0.00 & 0.00 & 0.00 & 0.00 & 0.00 & 0.00 & 1.80 \\
\hline Va. & Buchanan & 1.06 & 1.03 & 1.09 & 1.13 & 1.07 & 1.07 & 1.06 & 1.06 & 0.98 & 1.00 & 0.91 & 0.87 & 0.85 & 1.02 \\
\hline Va. & Dickenson & 1.07 & 1.07 & 1.25 & 1.28 & 1.30 & 1.44 & 1.32 & 1.21 & 1.17 & 0.99 & 0.94 & 0.91 & 1.02 & 1.10 \\
\hline Va. & Lee & 0.92 & 0.88 & 0.87 & 0.91 & 0.93 & 1.14 & 1.21 & 1.14 & 1.14 & 1.12 & 1.26 & 1.15 & 0.83 & 1.04 \\
\hline
\end{tabular}


Table 3. Weighted average sulfur content (in weight percent) of bituminous coal that was mined in the Appalachian basin for electrical power generation.-Continued [Data are shown by county. Data for 1983 through 1995 are from Attanasi and Milici (1998); data for 1996 through 2005 are from the COALdat database. nd, no data]

\begin{tabular}{|c|c|c|c|c|c|c|c|c|c|c|c|c|}
\hline State & County & 1996 & 1997 & 1998 & 1999 & 2000 & 2001 & 2002 & 2003 & 2004 & 2005 & $\begin{array}{l}\text { Weighted } \\
\text { average } \\
(1996-2005)\end{array}$ \\
\hline $\mathrm{Pa}$. & Fayette & 1.83 & 1.60 & 2.00 & 1.61 & 1.54 & 1.60 & 1.07 & 1.08 & 1.74 & 2.78 & 1.82 \\
\hline Pa. & Fulton & 2.12 & 0.00 & 0.00 & 0.00 & 0.00 & 0.00 & 0.00 & 0.00 & 0.00 & 0.00 & 2.12 \\
\hline Pa. & Greene & 1.81 & 1.81 & 1.79 & 1.92 & 1.89 & 1.88 & 2.00 & 2.18 & 2.06 & 1.96 & 1.92 \\
\hline Pa. & Huntingdon & nd & nd & nd & nd & nd & nd & nd & nd & nd & nd & No production \\
\hline Pa. & Indiana & 1.78 & 1.76 & 1.77 & 2.05 & 2.16 & 2.16 & 2.72 & 2.61 & 2.41 & 2.46 & 2.11 \\
\hline Pa. & Jefferson & 1.72 & 1.73 & 1.70 & 1.74 & 1.77 & 1.55 & 1.73 & 1.53 & 2.16 & 1.84 & 1.74 \\
\hline Pa. & Lawrence & 1.20 & 0.00 & 0.00 & 0.00 & 2.95 & 0.00 & 0.00 & 3.69 & 0.00 & 3.39 & 2.43 \\
\hline Pa. & Lycoming & 0.76 & 0.86 & 0.68 & 0.70 & 0.60 & 0.00 & 0.87 & 0.89 & 1.04 & 0.92 & 0.78 \\
\hline Pa. & Mercer & 0.00 & 0.00 & 0.00 & 0.00 & 0.00 & 0.00 & 0.00 & 6.40 & 5.42 & 0.00 & 5.93 \\
\hline $\mathrm{Pa}$. & Somerset & 1.91 & 2.11 & 2.11 & 1.97 & 1.97 & 2.05 & 2.08 & 2.21 & 2.33 & 1.87 & 2.06 \\
\hline Pa. & Tioga & 0.00 & 0.00 & 0.00 & 0.00 & 0.00 & 0.00 & 0.87 & 0.64 & 0.50 & 0.48 & 0.63 \\
\hline Pa. & Venango & 1.84 & 2.35 & 2.47 & 2.26 & 0.00 & 0.00 & 3.00 & 3.16 & 4.90 & 4.05 & 2.94 \\
\hline Pa. & Washington & 1.52 & 1.49 & 1.46 & 1.49 & 1.47 & 1.48 & 1.57 & 1.61 & 1.55 & 1.59 & 1.52 \\
\hline Pa. & Westmoreland & 2.03 & 2.14 & 2.32 & 2.17 & 2.34 & 2.30 & 0.00 & 0.00 & 0.71 & 1.43 & 2.11 \\
\hline Tenn. & Anderson & 1.53 & 1.19 & 1.50 & 1.30 & 0.00 & 0.00 & 0.00 & 0.84 & 0.89 & 1.42 & 1.35 \\
\hline Tenn. & Bledsoe & nd & nd & nd & nd & nd & nd & nd & nd & nd & nd & No production \\
\hline Tenn. & Campbell & 1.07 & 1.21 & 0.00 & 0.00 & 0.00 & 0.94 & 0.90 & 0.88 & 0.95 & 1.40 & 1.07 \\
\hline Tenn. & Claiborne & 1.50 & 1.50 & 1.40 & 1.37 & 1.30 & 1.37 & 1.33 & 1.26 & 1.28 & 1.32 & 1.34 \\
\hline Tenn. & Cumberland & 0.00 & 0.00 & 0.81 & 0.94 & 1.02 & 0.73 & 0.70 & 0.75 & 0.88 & 0.73 & 0.83 \\
\hline Tenn. & Fentress & 0.00 & 0.00 & 0.00 & 0.00 & 0.00 & 0.00 & 0.00 & 0.00 & 2.21 & 0.00 & 2.21 \\
\hline Tenn. & Grundy & 0.00 & 0.00 & 0.00 & 0.00 & 0.00 & 0.00 & 0.00 & 0.00 & 0.00 & 2.17 & 2.17 \\
\hline Tenn. & Marion & nd & nd & nd & nd & nd & nd & nd & nd & nd & nd & No production \\
\hline Tenn. & Morgan & 1.20 & 1.50 & 1.60 & 1.56 & 0.00 & 0.00 & 0.00 & 0.00 & 0.00 & 0.00 & 1.23 \\
\hline Tenn. & Rhea & nd & nd & nd & nd & nd & nd & nd & nd & nd & nd & No production \\
\hline Tenn. & Scott & 1.32 & 1.27 & 1.28 & 1.35 & 1.28 & 1.04 & 0.00 & 0.00 & 1.10 & 1.66 & 1.31 \\
\hline Tenn. & Sequatchie & 0.88 & 0.86 & 0.88 & 0.89 & 0.75 & 0.00 & 0.00 & 0.00 & 0.00 & 0.00 & 0.86 \\
\hline Tenn. & Van Buren & nd & nd & nd & nd & nd & nd & nd & nd & nd & nd & No production \\
\hline Va. & Buchanan & 0.87 & 0.83 & 0.80 & 0.83 & 0.77 & 0.80 & 0.84 & 0.86 & 0.87 & 0.85 & 0.83 \\
\hline Va. & Dickenson & 1.06 & 1.09 & 0.93 & 1.13 & 1.03 & 1.01 & 0.99 & 1.10 & 1.11 & 1.35 & 1.08 \\
\hline Va. & Lee & 1.03 & 1.06 & 0.97 & 0.93 & 0.74 & 0.76 & 0.76 & 0.77 & 0.84 & 0.88 & 0.84 \\
\hline
\end{tabular}


Table 3. Weighted average sulfur content (in weight percent) of bituminous coal that was mined in the Appalachian basin for electrical power generation.-Continued [Data are shown by county. Data for 1983 through 1995 are from Attanasi and Milici (1998); data for 1996 through 2005 are from the COALdat database. nd, no data]

\begin{tabular}{|c|c|c|c|c|c|c|c|c|c|c|c|c|c|c|c|}
\hline State & County & 1983 & 1984 & 1985 & 1986 & 1987 & 1988 & 1989 & 1990 & 1991 & 1992 & 1993 & 1994 & 1995 & $\begin{array}{l}\text { Weighted } \\
\text { average } \\
\text { (1983-1995) }\end{array}$ \\
\hline Va. & Russell & 0.71 & 0.76 & 0.74 & 0.75 & 0.76 & 0.76 & 0.75 & 0.77 & 0.81 & 0.75 & 0.77 & 0.70 & 0.75 & 0.75 \\
\hline Va. & Scott & 0.00 & 0.00 & 0.00 & 1.00 & 0.00 & 0.00 & 0.00 & 0.00 & 0.00 & 0.00 & 0.00 & 0.00 & 0.00 & 1.00 \\
\hline Va. & Tazewell & 0.79 & 0.76 & 0.92 & 0.85 & 0.79 & 0.76 & 1.22 & 0.56 & 0.00 & 0.00 & 0.00 & 0.00 & 0.00 & 0.85 \\
\hline Va. & Wise & 0.97 & 1.04 & 1.08 & 1.07 & 1.12 & 1.13 & 1.20 & 1.23 & 1.22 & 1.16 & 1.06 & 1.12 & 1.13 & 1.12 \\
\hline W. Va. & Barbour & 2.65 & 2.32 & 2.19 & 2.04 & 2.21 & 2.06 & 1.81 & 1.82 & 1.82 & 1.83 & 1.80 & 1.76 & 1.19 & 2.00 \\
\hline W. Va. & Boone & 0.82 & 0.79 & 0.78 & 0.78 & 0.78 & 0.78 & 0.79 & 0.82 & 0.82 & 0.84 & 0.86 & 0.82 & 0.80 & 0.81 \\
\hline W. Va. & Braxton & 0.80 & 0.78 & 0.72 & 0.81 & 0.79 & 0.82 & 0.92 & 0.84 & 0.89 & 0.00 & 1.02 & 0.00 & 0.00 & 0.81 \\
\hline W. Va. & Brooke & 3.27 & 3.39 & 3.22 & 3.32 & 3.24 & 3.31 & 3.22 & 2.95 & 2.85 & 2.88 & 2.83 & 3.27 & 3.27 & 3.12 \\
\hline W. Va. & Clay & 0.00 & 0.84 & 0.00 & 1.77 & 0.89 & 1.05 & 1.35 & 1.16 & 1.10 & 1.11 & 0.82 & 0.77 & 0.72 & 0.96 \\
\hline W. Va. & Fayette & 0.78 & 1.18 & 0.68 & 0.72 & 1.02 & 1.33 & 1.27 & 1.04 & 1.19 & 1.30 & 1.23 & 0.93 & 0.88 & 1.11 \\
\hline W. Va. & Gilmer & 0.77 & 0.71 & 0.00 & 0.00 & 2.79 & 2.61 & 0.00 & 0.00 & 0.00 & 0.00 & 0.00 & 0.00 & 0.00 & 2.40 \\
\hline W. Va. & Grant & 1.78 & 1.77 & 1.77 & 1.76 & 1.75 & 1.73 & 1.75 & 1.70 & 1.63 & 1.64 & 1.62 & 1.73 & 1.65 & 1.71 \\
\hline W. Va. & Greenbrier & 1.16 & 1.22 & 1.05 & 1.15 & 1.06 & 0.89 & 0.80 & 0.00 & 0.62 & 0.93 & 0.97 & 1.02 & 0.88 & 1.08 \\
\hline W. Va. & Harrison & 3.22 & 3.19 & 3.26 & 3.16 & 3.07 & 3.02 & 3.08 & 3.17 & 3.12 & 3.14 & 2.97 & 3.10 & 3.57 & 3.17 \\
\hline W. Va. & Kanawha & 0.74 & 0.76 & 0.77 & 0.78 & 0.79 & 0.77 & 0.82 & 0.89 & 0.93 & 0.88 & 0.92 & 0.82 & 0.83 & 0.83 \\
\hline W. Va. & Lewis & 2.73 & 2.80 & 2.86 & 2.88 & 2.80 & 3.30 & 3.03 & 1.62 & 0.00 & 0.00 & 2.43 & 2.68 & 2.99 & 2.86 \\
\hline W. Va. & Lincoln & 0.00 & 0.00 & 0.00 & 0.00 & 0.68 & 0.80 & 0.77 & 0.70 & 0.84 & 0.79 & 0.78 & 0.85 & 0.73 & 0.79 \\
\hline W. Va. & Logan & 0.67 & 0.66 & 0.66 & 0.78 & 0.79 & 0.80 & 0.74 & 0.73 & 0.71 & 0.71 & 0.69 & 0.70 & 0.70 & 0.72 \\
\hline W. Va. & McDowell & 0.83 & 0.74 & 0.73 & 0.64 & 0.81 & 0.72 & 0.98 & 0.79 & 0.96 & 0.66 & 0.95 & 0.61 & 1.03 & 0.81 \\
\hline W. Va. & Marion & 2.47 & 2.06 & 1.99 & 1.86 & 1.80 & 1.70 & 1.65 & 1.71 & 1.86 & 1.74 & 2.26 & 1.70 & 1.91 & 1.96 \\
\hline W. Va. & Marshall & 3.81 & 3.83 & 3.88 & 3.84 & 3.91 & 3.91 & 4.04 & 4.13 & 4.11 & 3.95 & 3.68 & 3.88 & 3.72 & 3.92 \\
\hline W. Va. & Mason & 2.45 & 0.00 & 0.00 & 0.00 & 2.85 & 2.85 & 2.93 & 2.48 & 2.84 & 2.59 & 3.59 & 3.54 & 0.00 & 2.87 \\
\hline W. Va. & Mercer & 0.00 & 0.00 & 0.71 & 0.57 & 0.65 & 0.00 & 0.00 & 0.00 & 0.00 & 0.00 & 0.00 & 0.00 & 0.00 & 0.66 \\
\hline W. Va. & Mineral & 1.55 & 1.74 & 1.78 & 1.49 & 1.38 & 1.52 & 1.63 & 1.69 & 1.67 & 1.75 & 1.80 & 1.71 & 1.66 & 1.67 \\
\hline W. Va. & Mingo & 0.82 & 0.75 & 0.68 & 0.73 & 0.74 & 0.72 & 0.75 & 0.77 & 0.78 & 0.76 & 0.78 & 0.78 & 0.74 & 0.76 \\
\hline W. Va. & Monongalia & 2.42 & 2.58 & 2.33 & 2.37 & 2.43 & 2.40 & 2.26 & 2.35 & 2.32 & 2.26 & 2.20 & 2.28 & 2.31 & 2.35 \\
\hline W. Va. & Nicholas & 0.84 & 0.89 & 0.88 & 0.84 & 0.92 & 0.90 & 0.87 & 0.94 & 0.89 & 0.95 & 1.10 & 0.92 & 0.86 & 0.90 \\
\hline W. Va. & Ohio & 1.60 & 0.00 & 2.88 & 3.02 & 2.69 & 3.03 & 2.94 & 2.98 & 2.90 & 2.87 & 3.12 & 3.68 & 2.78 & 3.16 \\
\hline W. Va. & Preston & 1.82 & 1.86 & 1.57 & 1.54 & 1.65 & 1.71 & 1.59 & 1.60 & 1.50 & 1.51 & 1.47 & 1.45 & 1.47 & 1.62 \\
\hline W. Va. & Raleigh & 0.92 & 1.05 & 0.93 & 1.01 & 0.00 & 0.00 & 0.83 & 0.69 & 0.80 & 0.80 & 0.85 & 0.00 & 0.00 & 0.86 \\
\hline
\end{tabular}


Table 3. Weighted average sulfur content (in weight percent) of bituminous coal that was mined in the Appalachian basin for electrical power generation.-Continued [Data are shown by county. Data for 1983 through 1995 are from Attanasi and Milici (1998); data for 1996 through 2005 are from the COALdat database. nd, no data]

\begin{tabular}{|c|c|c|c|c|c|c|c|c|c|c|c|c|}
\hline State & County & 1996 & 1997 & 1998 & 1999 & 2000 & 2001 & 2002 & 2003 & 2004 & 2005 & $\begin{array}{l}\text { Weighted } \\
\text { average } \\
(1996-2005)\end{array}$ \\
\hline Va. & Russell & 0.70 & 0.74 & 0.74 & 0.73 & 0.70 & 0.75 & 0.73 & 0.70 & 0.74 & 0.85 & 0.74 \\
\hline Va. & Scott & nd & nd & nd & nd & nd & nd & nd & nd & nd & nd & No production \\
\hline Va. & Tazewell & 1.18 & 0.00 & 0.00 & 0.00 & 0.00 & 0.00 & 0.59 & 0.56 & 0.52 & 0.53 & 0.58 \\
\hline Va. & Wise & 1.14 & 1.17 & 1.10 & 1.08 & 1.07 & 1.11 & 1.01 & 1.02 & 1.01 & 1.01 & 1.06 \\
\hline W. Va. & Barbour & 1.83 & 2.35 & 2.47 & 2.04 & 1.72 & 1.31 & 1.74 & 2.41 & 1.66 & 2.54 & 2.04 \\
\hline W. Va. & Boone & 0.81 & 0.78 & 0.78 & 0.79 & 0.78 & 0.78 & 0.78 & 0.82 & 0.83 & 0.80 & 0.79 \\
\hline W. Va. & Braxton & nd & nd & nd & nd & nd & nd & nd & nd & nd & nd & No production \\
\hline W. Va. & Brooke & 3.64 & 3.76 & 4.05 & 3.72 & 3.76 & 3.68 & 3.79 & 0.00 & 0.00 & 0.00 & 3.79 \\
\hline W. Va. & Clay & 0.79 & 0.82 & 0.85 & 0.89 & 0.75 & 0.74 & 0.79 & 0.77 & 0.74 & 0.77 & 0.80 \\
\hline W. Va. & Fayette & 1.03 & 1.02 & 1.05 & 1.09 & 1.20 & 1.14 & 0.74 & 0.64 & 1.20 & 0.84 & 1.03 \\
\hline W. Va. & Gilmer & nd & nd & nd & nd & nd & nd & nd & nd & nd & nd & No production \\
\hline W. Va. & Grant & 1.61 & 1.53 & 1.61 & 1.74 & 1.71 & 1.60 & 1.68 & 1.67 & 1.81 & 1.75 & 1.64 \\
\hline W. Va. & Greenbrier & 0.00 & 0.00 & 0.00 & 0.89 & 0.86 & 0.93 & 0.85 & 0.53 & 0.00 & 0.00 & 0.88 \\
\hline W. Va. & Harrison & 3.38 & 3.48 & 3.44 & 3.34 & 3.27 & 3.27 & 3.28 & 3.25 & 3.51 & 3.54 & 3.38 \\
\hline W. Va. & Kanawha & 0.79 & 0.76 & 0.79 & 0.78 & 0.76 & 0.75 & 0.73 & 0.72 & 0.73 & 0.75 & 0.76 \\
\hline W. Va. & Lewis & nd & nd & nd & nd & nd & nd & nd & nd & nd & nd & No production \\
\hline W. Va. & Lincoln & 0.60 & 0.68 & 0.86 & 1.20 & 1.25 & 0.93 & 0.86 & 0.90 & 0.75 & 0.84 & 0.90 \\
\hline W. Va. & Logan & 0.73 & 0.72 & 0.71 & 0.80 & 0.69 & 0.72 & 0.73 & 0.74 & 0.75 & 0.75 & 0.73 \\
\hline W. Va. & McDowell & 0.71 & 0.00 & 0.00 & 0.71 & 0.79 & 0.80 & 0.80 & 0.70 & 0.80 & 0.83 & 0.78 \\
\hline W. Va. & Marion & 2.50 & 2.08 & 2.14 & 2.78 & 0.00 & 2.11 & 3.13 & 2.04 & 2.22 & 2.33 & 2.26 \\
\hline W. Va. & Marshall & 3.71 & 3.63 & 3.65 & 3.67 & 3.53 & 3.36 & 3.14 & 2.61 & 2.29 & 2.40 & 3.28 \\
\hline W. Va. & Mason & 0.00 & 0.00 & 0.00 & 0.00 & 0.78 & 0.00 & 0.00 & 0.00 & 0.70 & 0.00 & 0.75 \\
\hline W. Va. & Mercer & 0.00 & 0.00 & 0.00 & 0.00 & 0.00 & 0.00 & 1.12 & 0.00 & 0.00 & 0.00 & 1.12 \\
\hline W. Va. & Mineral & 1.61 & 0.00 & 0.00 & 0.00 & 1.69 & 0.00 & 0.00 & 0.00 & 0.00 & 2.03 & 1.67 \\
\hline W. Va. & Mingo & 0.78 & 0.78 & 0.77 & 0.74 & 0.72 & 0.73 & 0.74 & 0.82 & 0.80 & 0.72 & 0.76 \\
\hline W. Va. & Monongalia & 2.18 & 2.12 & 2.26 & 2.26 & 2.27 & 2.28 & 2.17 & 2.17 & 2.35 & 2.21 & 2.22 \\
\hline W. Va. & Nicholas & 0.90 & 0.73 & 0.82 & 1.02 & 0.84 & 0.79 & 1.03 & 0.97 & 0.93 & 0.99 & 0.92 \\
\hline W. Va. & Ohio & 0.00 & 0.00 & 0.00 & 0.00 & 4.17 & 0.00 & 0.00 & 0.00 & 0.00 & 0.00 & 4.17 \\
\hline W. Va. & Preston & 1.41 & 1.48 & 1.49 & 1.37 & 1.47 & 1.46 & 1.69 & 1.61 & 1.64 & 1.58 & 1.53 \\
\hline W. Va. & Raleigh & 0.76 & 1.04 & 0.99 & 1.01 & 1.10 & 1.07 & 0.88 & 0.98 & 1.36 & 1.30 & 1.11 \\
\hline
\end{tabular}


Table 3. Weighted average sulfur content (in weight percent) of bituminous coal that was mined in the Appalachian basin for electrical power generation.-Continued [Data are shown by county. Data for 1983 through 1995 are from Attanasi and Milici (1998); data for 1996 through 2005 are from the COALdat database. nd, no data]

\begin{tabular}{|c|c|c|c|c|c|c|c|c|c|c|c|c|c|c|c|}
\hline State & County & 1983 & 1984 & 1985 & 1986 & 1987 & 1988 & 1989 & 1990 & 1991 & 1992 & 1993 & 1994 & 1995 & $\begin{array}{l}\text { Weighted } \\
\text { average } \\
\text { (1983-1995) }\end{array}$ \\
\hline W. Va. & Randolph & 1.10 & 1.11 & 1.07 & 1.14 & 1.17 & 1.24 & 1.24 & 1.45 & 1.37 & 1.08 & 1.56 & 0.85 & 1.10 & 1.18 \\
\hline W. Va. & Taylor & 0.00 & 0.00 & 1.39 & 1.41 & 0.00 & 1.53 & 1.59 & 1.66 & 0.00 & 1.59 & 1.70 & 0.00 & 0.00 & 1.52 \\
\hline W. Va. & Tucker & 2.03 & 1.80 & 1.92 & 1.60 & 0.00 & 0.00 & 0.00 & 0.00 & 0.00 & 0.00 & 0.00 & 0.00 & 0.00 & 1.77 \\
\hline W. Va. & Upshur & 1.19 & 1.17 & 1.19 & 1.18 & 1.53 & 2.58 & 2.28 & 2.07 & 2.63 & 2.88 & 2.19 & 2.41 & 2.51 & 1.63 \\
\hline W. Va. & Wayne & 0.98 & 0.00 & 2.25 & 1.16 & 1.83 & 1.08 & 0.84 & 0.69 & 0.68 & 0.84 & 0.89 & 0.86 & 0.83 & 0.86 \\
\hline W. Va. & Webster & 0.66 & 0.69 & 0.76 & 0.77 & 0.83 & 0.85 & 0.82 & 0.82 & 0.82 & 0.85 & 0.96 & 0.84 & 0.84 & 0.84 \\
\hline W. Va. & Wyoming & 0.98 & 1.11 & 0.94 & 0.96 & 0.76 & 0.77 & 0.73 & 0.00 & 0.00 & 0.00 & 0.65 & 0.68 & 0.74 & 0.92 \\
\hline
\end{tabular}


Table 3. Weighted average sulfur content (in weight percent) of bituminous coal that was mined in the Appalachian basin for electrical power generation.-Continued [Data are shown by county. Data for 1983 through 1995 are from Attanasi and Milici (1998); data for 1996 through 2005 are from the COALdat database. nd, no data]

\begin{tabular}{|c|c|c|c|c|c|c|c|c|c|c|c|c|}
\hline State & County & 1996 & 1997 & 1998 & 1999 & 2000 & 2001 & 2002 & 2003 & 2004 & 2005 & $\begin{array}{l}\text { Weighted } \\
\text { average } \\
\text { (1996-2005) }\end{array}$ \\
\hline W. Va. & Randolph & 0.00 & 0.00 & 0.00 & 0.00 & 0.00 & 0.00 & 0.00 & 0.72 & 0.00 & 0.00 & 0.72 \\
\hline W. Va. & Taylor & nd & nd & nd & nd & nd & nd & nd & nd & nd & nd & No production \\
\hline W. Va. & Tucker & nd & nd & nd & nd & nd & nd & nd & nd & nd & nd & No production \\
\hline W. Va. & Upshur & 2.42 & 2.02 & 1.61 & 1.49 & 1.53 & 1.55 & 2.20 & 2.42 & 2.09 & 1.61 & 1.80 \\
\hline W. Va. & Wayne & 0.79 & 0.79 & 0.80 & 0.81 & 0.78 & 0.79 & 0.81 & 0.77 & 0.80 & 0.84 & 0.80 \\
\hline W. Va. & Webster & 0.83 & 0.86 & 0.88 & 0.92 & 0.96 & 0.95 & 1.07 & 1.09 & 1.12 & 1.09 & 0.97 \\
\hline W. Va. & Wyoming & 1.08 & 0.65 & 0.74 & 0.71 & 0.79 & 0.75 & 0.98 & 0.85 & 0.80 & 0.93 & 0.84 \\
\hline
\end{tabular}


Table 4. Potential $\mathrm{SO}_{2}$ emissions (in pounds of $\mathrm{SO}_{2}$ per million British thermal units) of bituminous coal that was mined in the Appalachian basin for electrical power generation.

[Data are shown by county. Data for 1983 through 1995 were calculated from data given in tables 1-3; data for 1996 through 2005 are from the COALdat database. n/a, not available]

\begin{tabular}{|c|c|c|c|c|c|c|c|c|c|c|c|c|c|c|c|}
\hline State & County & 1983 & 1984 & 1985 & 1986 & 1987 & 1988 & 1989 & 1990 & 1991 & 1992 & 1993 & 1994 & 1995 & $\begin{array}{c}\text { Weighted average } \\
\text { (1983-1995) }\end{array}$ \\
\hline Ala. & Bibb & 0.00 & 0.00 & 0.00 & 0.00 & 0.00 & 0.00 & 0.00 & 0.00 & 0.00 & 0.00 & 0.00 & 0.00 & 1.42 & 1.42 \\
\hline Ala. & Blount & 0.00 & 3.85 & 3.71 & 2.89 & 1.69 & 0.00 & 0.00 & 4.09 & 3.25 & 0.00 & 0.00 & 0.00 & 0.00 & 3.61 \\
\hline Ala. & Cullman & 1.08 & 1.08 & 0.00 & 2.50 & 0.00 & 0.00 & 0.00 & 0.00 & 0.00 & 0.00 & 0.00 & 0.00 & 0.00 & 1.12 \\
\hline Ala. & De Kalb & 0.00 & 0.00 & 0.00 & 1.87 & 0.00 & 0.00 & 0.00 & 0.00 & 0.00 & 0.00 & 0.00 & 0.00 & 0.00 & 1.87 \\
\hline Ala. & Fayette & 3.85 & 3.97 & 3.67 & 3.37 & 3.39 & 3.63 & 3.40 & 3.53 & 3.77 & 3.49 & 3.10 & 3.03 & 2.99 & 3.45 \\
\hline Ala. & Jackson & 1.85 & 1.84 & 1.98 & 0.85 & 1.01 & 0.00 & 0.00 & 0.00 & 0.00 & 0.00 & 0.00 & 2.84 & 0.00 & 2.00 \\
\hline Ala. & Jefferson & 2.00 & 2.34 & 2.17 & 2.13 & 1.91 & 2.00 & 2.24 & 2.11 & 1.80 & 2.01 & 1.86 & 1.75 & 1.65 & 1.98 \\
\hline Ala. & Lamar & 0.00 & 0.00 & 0.00 & 0.00 & 0.00 & 2.27 & 2.27 & 0.00 & 0.00 & 0.00 & 0.00 & 0.00 & 0.00 & 2.27 \\
\hline Ala. & Marion & 0.00 & 2.63 & 1.97 & 1.92 & 1.82 & 2.01 & 2.27 & 2.41 & 3.11 & 2.70 & 2.49 & 2.24 & 0.00 & 2.21 \\
\hline Ala. & Marshall & 3.30 & 3.17 & 0.00 & 0.00 & 0.00 & 0.00 & 0.00 & 0.00 & 0.00 & 0.00 & 0.00 & 0.00 & 0.00 & 3.25 \\
\hline Ala. & St. Clair & 0.00 & 0.00 & 2.15 & 0.00 & 1.89 & 2.36 & 0.00 & 0.00 & 0.00 & 0.00 & 0.00 & 0.00 & 0.00 & 2.11 \\
\hline Ala. & Shelby & 0.00 & 0.00 & 0.00 & 0.00 & 0.00 & 0.00 & 0.00 & 0.00 & 0.00 & 0.00 & 0.00 & 0.00 & 1.33 & 1.33 \\
\hline Ala. & Tuscaloosa & 3.05 & 3.15 & 2.39 & 2.42 & 2.22 & 2.27 & 2.19 & 1.87 & 1.67 & 1.78 & 1.58 & 1.59 & 1.72 & 1.92 \\
\hline Ala. & Walker & 2.15 & 2.02 & 1.93 & 2.00 & 1.83 & 1.85 & 1.84 & 1.90 & 1.98 & 1.84 & 1.74 & 1.75 & 1.65 & 1.90 \\
\hline Ala. & Winston & 0.00 & 0.00 & 2.24 & 1.58 & 2.29 & 1.64 & 2.41 & 2.43 & 2.20 & 2.24 & 1.94 & 2.10 & 0.00 & 2.17 \\
\hline Ga. & Dade & 2.40 & 2.43 & 0.00 & 0.00 & 0.00 & 0.00 & 0.00 & 0.00 & 0.00 & 0.00 & 0.00 & 0.00 & 0.00 & 2.43 \\
\hline Ky. & Bell & 2.10 & 2.09 & 2.07 & 2.00 & 2.05 & 2.06 & 1.97 & 2.15 & 2.03 & 1.91 & 2.01 & 2.09 & 2.16 & 2.05 \\
\hline Ку. & Boyd & 2.08 & 2.20 & 4.30 & 3.04 & 1.64 & 1.00 & 1.52 & 1.09 & 1.15 & 1.46 & 1.37 & 1.36 & 1.21 & 1.96 \\
\hline Ky. & Breathitt & 1.48 & 1.44 & 1.48 & 1.53 & 1.76 & 1.78 & 1.74 & 1.84 & 1.93 & 1.79 & 1.92 & 1.81 & 1.59 & 1.68 \\
\hline Ky. & Carter & 3.55 & 1.93 & 1.63 & 1.72 & 1.87 & 1.76 & 1.34 & 2.26 & 2.70 & 2.47 & 0.00 & 2.72 & 0.00 & 2.01 \\
\hline Ky. & Clay & 2.50 & 2.61 & 2.50 & 2.55 & 2.60 & 2.57 & 2.29 & 2.21 & 2.31 & 1.72 & 2.29 & 2.16 & 1.82 & 2.50 \\
\hline Ky. & Clinton & 0.00 & 0.00 & 1.26 & 0.00 & 0.00 & 0.00 & 0.00 & 0.00 & 0.00 & 0.00 & 0.00 & 0.00 & 0.00 & 1.26 \\
\hline Ky. & Elliott & 2.63 & 2.93 & 2.91 & 2.60 & 2.83 & 0.00 & 2.35 & 2.03 & 2.12 & 0.00 & 0.00 & 0.00 & 0.00 & 2.73 \\
\hline Ky. & Estill & 5.85 & 0.00 & 0.00 & 1.13 & 0.00 & 0.00 & 0.00 & 0.00 & 0.00 & 0.00 & 1.21 & 2.35 & 1.48 & 1.41 \\
\hline Ky. & Floyd & 1.71 & 1.73 & 1.78 & 1.75 & 1.79 & 1.73 & 1.69 & 1.60 & 1.58 & 1.54 & 1.77 & 1.77 & 1.55 & 1.69 \\
\hline Ky. & Greenup & 3.06 & 2.84 & 5.06 & 5.39 & 3.80 & 4.17 & 3.24 & 4.23 & 4.47 & 4.16 & 3.69 & 3.28 & 1.29 & 3.67 \\
\hline Ку. & Harlan & 1.96 & 1.85 & 1.64 & 1.65 & 1.67 & 1.71 & 1.88 & 1.87 & 1.78 & 1.71 & 1.80 & 1.67 & 1.62 & 1.75 \\
\hline Ky. & Jackson & 1.72 & 2.22 & 2.35 & 3.19 & 0.00 & 1.63 & 1.67 & 2.48 & 2.84 & 3.79 & 0.00 & 1.55 & 1.49 & 2.13 \\
\hline Ky. & Johnson & 2.04 & 2.04 & 1.75 & 1.94 & 1.96 & 2.09 & 2.26 & 2.05 & 2.02 & 2.11 & 2.19 & 2.35 & 2.06 & 2.06 \\
\hline Ку. & Knott & 1.94 & 1.66 & 1.75 & 1.81 & 1.42 & 1.26 & 1.95 & 1.97 & 1.80 & 1.68 & 1.83 & 1.69 & 1.57 & 1.72 \\
\hline
\end{tabular}


Table 4. Potential $\mathrm{SO}_{2}$ emissions (in pounds of $\mathrm{SO}_{2}$ per million British thermal units) of bituminous coal that was mined in the Appalachian basin for electrical power generation.-Continued

[Data are shown by county. Data for 1983 through 1995 were calculated from data given in tables 1-3; data for 1996 through 2005 are from the COALdat database. n/a, not available]

\begin{tabular}{|c|c|c|c|c|c|c|c|c|c|c|c|c|}
\hline State & County & 1996 & 1997 & 1998 & 1999 & 2000 & 2001 & 2002 & 2003 & 2004 & 2005 & $\begin{array}{l}\text { Weighted average } \\
\text { (1996-2005) }\end{array}$ \\
\hline Ala. & Bibb & 3.04 & 1.57 & 1.64 & 0.00 & 0.00 & 0.00 & 0.00 & 0.00 & 2.94 & 2.94 & 2.49 \\
\hline Ala. & Blount & $\mathrm{n} / \mathrm{a}$ & $\mathrm{n} / \mathrm{a}$ & $\mathrm{n} / \mathrm{a}$ & $\mathrm{n} / \mathrm{a}$ & $\mathrm{n} / \mathrm{a}$ & $\mathrm{n} / \mathrm{a}$ & $\mathrm{n} / \mathrm{a}$ & $\mathrm{n} / \mathrm{a}$ & $\mathrm{n} / \mathrm{a}$ & $\mathrm{n} / \mathrm{a}$ & No production \\
\hline Ala. & Cullman & $\mathrm{n} / \mathrm{a}$ & $\mathrm{n} / \mathrm{a}$ & $\mathrm{n} / \mathrm{a}$ & $\mathrm{n} / \mathrm{a}$ & $\mathrm{n} / \mathrm{a}$ & $\mathrm{n} / \mathrm{a}$ & $\mathrm{n} / \mathrm{a}$ & $\mathrm{n} / \mathrm{a}$ & $\mathrm{n} / \mathrm{a}$ & $\mathrm{n} / \mathrm{a}$ & No production \\
\hline Ala. & De Kalb & $\mathrm{n} / \mathrm{a}$ & $\mathrm{n} / \mathrm{a}$ & $\mathrm{n} / \mathrm{a}$ & $\mathrm{n} / \mathrm{a}$ & $\mathrm{n} / \mathrm{a}$ & $\mathrm{n} / \mathrm{a}$ & $\mathrm{n} / \mathrm{a}$ & $\mathrm{n} / \mathrm{a}$ & $\mathrm{n} / \mathrm{a}$ & $\mathrm{n} / \mathrm{a}$ & No production \\
\hline Ala. & Fayette & 3.01 & 0.00 & 0.00 & 0.00 & 0.00 & 2.64 & 2.90 & 0.00 & 0.00 & 0.00 & 2.73 \\
\hline Ala. & Jackson & 0.00 & 0.00 & 0.00 & 0.00 & 1.52 & 0.00 & 0.00 & 1.43 & 1.43 & 1.11 & 1.29 \\
\hline Ala. & Jefferson & 1.49 & 1.23 & 1.17 & 1.26 & 1.18 & 1.28 & 1.22 & 1.23 & 1.74 & 2.09 & 1.36 \\
\hline Ala. & Lamar & $\mathrm{n} / \mathrm{a}$ & $\mathrm{n} / \mathrm{a}$ & $\mathrm{n} / \mathrm{a}$ & $\mathrm{n} / \mathrm{a}$ & $\mathrm{n} / \mathrm{a}$ & $\mathrm{n} / \mathrm{a}$ & $\mathrm{n} / \mathrm{a}$ & $\mathrm{n} / \mathrm{a}$ & $\mathrm{n} / \mathrm{a}$ & $\mathrm{n} / \mathrm{a}$ & No production \\
\hline Ala. & Marion & $\mathrm{n} / \mathrm{a}$ & $\mathrm{n} / \mathrm{a}$ & $\mathrm{n} / \mathrm{a}$ & $\mathrm{n} / \mathrm{a}$ & $\mathrm{n} / \mathrm{a}$ & $\mathrm{n} / \mathrm{a}$ & $\mathrm{n} / \mathrm{a}$ & $\mathrm{n} / \mathrm{a}$ & $\mathrm{n} / \mathrm{a}$ & $\mathrm{n} / \mathrm{a}$ & No production \\
\hline Ala. & Marshall & $\mathrm{n} / \mathrm{a}$ & $\mathrm{n} / \mathrm{a}$ & $\mathrm{n} / \mathrm{a}$ & $\mathrm{n} / \mathrm{a}$ & $\mathrm{n} / \mathrm{a}$ & $\mathrm{n} / \mathrm{a}$ & $\mathrm{n} / \mathrm{a}$ & $\mathrm{n} / \mathrm{a}$ & $\mathrm{n} / \mathrm{a}$ & $\mathrm{n} / \mathrm{a}$ & No production \\
\hline Ala. & St. Clair & $\mathrm{n} / \mathrm{a}$ & $\mathrm{n} / \mathrm{a}$ & $\mathrm{n} / \mathrm{a}$ & $\mathrm{n} / \mathrm{a}$ & $\mathrm{n} / \mathrm{a}$ & $\mathrm{n} / \mathrm{a}$ & $\mathrm{n} / \mathrm{a}$ & $\mathrm{n} / \mathrm{a}$ & $\mathrm{n} / \mathrm{a}$ & $\mathrm{n} / \mathrm{a}$ & No production \\
\hline Ala. & Shelby & 1.11 & 1.07 & 1.19 & 0.00 & 1.18 & 1.09 & 0.00 & 0.00 & 0.00 & 0.00 & 1.11 \\
\hline Ala. & Tuscaloosa & 2.37 & 2.04 & 1.91 & 1.90 & 1.98 & 2.00 & 2.47 & 2.41 & 2.73 & 2.86 & 2.20 \\
\hline Ala. & Walker & 2.10 & 2.73 & 2.75 & 2.26 & 2.61 & 2.34 & 2.70 & 2.58 & 2.72 & 2.86 & 2.57 \\
\hline Ala. & Winston & $\mathrm{n} / \mathrm{a}$ & $\mathrm{n} / \mathrm{a}$ & $\mathrm{n} / \mathrm{a}$ & $\mathrm{n} / \mathrm{a}$ & $\mathrm{n} / \mathrm{a}$ & $\mathrm{n} / \mathrm{a}$ & $\mathrm{n} / \mathrm{a}$ & $\mathrm{n} / \mathrm{a}$ & $\mathrm{n} / \mathrm{a}$ & $\mathrm{n} / \mathrm{a}$ & No production \\
\hline Ga. & Dade & $\mathrm{n} / \mathrm{a}$ & $\mathrm{n} / \mathrm{a}$ & $\mathrm{n} / \mathrm{a}$ & $\mathrm{n} / \mathrm{a}$ & $\mathrm{n} / \mathrm{a}$ & $\mathrm{n} / \mathrm{a}$ & $\mathrm{n} / \mathrm{a}$ & $\mathrm{n} / \mathrm{a}$ & $\mathrm{n} / \mathrm{a}$ & $\mathrm{n} / \mathrm{a}$ & No production \\
\hline Ky. & Bell & 2.12 & 2.25 & 2.26 & 2.12 & 2.01 & 2.18 & 1.63 & 1.67 & 1.49 & 1.66 & 2.04 \\
\hline Ky. & Boyd & 1.16 & 1.17 & 1.42 & 1.25 & 1.44 & 1.39 & 1.74 & 1.66 & 1.62 & 1.63 & 1.50 \\
\hline Ky. & Breathitt & 1.82 & 2.45 & 2.40 & 2.13 & 1.67 & 1.90 & 2.61 & 2.43 & 2.04 & 2.48 & 2.22 \\
\hline Ky. & Carter & 0.00 & 0.00 & 1.37 & 0.00 & 0.00 & 0.00 & 0.00 & 0.00 & 0.00 & 0.00 & 1.37 \\
\hline Ky. & Clay & 1.92 & 1.85 & 1.80 & 1.84 & 2.23 & 2.03 & 1.93 & 2.02 & 2.78 & 2.34 & 2.05 \\
\hline Ky. & Clinton & $\mathrm{n} / \mathrm{a}$ & $\mathrm{n} / \mathrm{a}$ & $\mathrm{n} / \mathrm{a}$ & $\mathrm{n} / \mathrm{a}$ & $\mathrm{n} / \mathrm{a}$ & $\mathrm{n} / \mathrm{a}$ & $\mathrm{n} / \mathrm{a}$ & $\mathrm{n} / \mathrm{a}$ & $\mathrm{n} / \mathrm{a}$ & $\mathrm{n} / \mathrm{a}$ & No production \\
\hline Ky. & Elliott & $\mathrm{n} / \mathrm{a}$ & $\mathrm{n} / \mathrm{a}$ & $\mathrm{n} / \mathrm{a}$ & $\mathrm{n} / \mathrm{a}$ & $\mathrm{n} / \mathrm{a}$ & $\mathrm{n} / \mathrm{a}$ & $\mathrm{n} / \mathrm{a}$ & $\mathrm{n} / \mathrm{a}$ & $\mathrm{n} / \mathrm{a}$ & $\mathrm{n} / \mathrm{a}$ & No production \\
\hline Ky. & Estill & 1.41 & 1.28 & 0.00 & 1.45 & 2.06 & 1.78 & 1.10 & 1.11 & 1.14 & 0.00 & 1.73 \\
\hline Ку. & Floyd & 1.64 & 1.80 & 1.53 & 1.56 & 1.54 & 1.72 & 1.88 & 1.81 & 2.13 & 2.09 & 1.71 \\
\hline Ky. & Greenup & 1.30 & 1.26 & 1.47 & 1.44 & 1.45 & 1.37 & 0.00 & 0.00 & 0.00 & 0.00 & 1.38 \\
\hline Ky. & Harlan & 1.75 & 1.68 & 1.64 & 1.63 & 1.62 & 1.68 & 1.68 & 1.62 & 1.64 & 1.58 & 1.65 \\
\hline Ky. & Jackson & 0.00 & 0.00 & 0.00 & 0.00 & 0.00 & 0.00 & 1.33 & 0.00 & 0.00 & 0.00 & 1.33 \\
\hline Ky. & Johnson & 2.14 & 2.26 & 2.17 & 2.16 & 1.87 & 1.86 & 1.78 & 1.71 & 1.76 & 1.80 & 2.01 \\
\hline Ky. & Knott & 1.79 & 1.85 & 1.81 & 1.80 & 1.64 & 1.73 & 1.74 & 1.81 & 1.93 & 1.83 & 1.79 \\
\hline
\end{tabular}




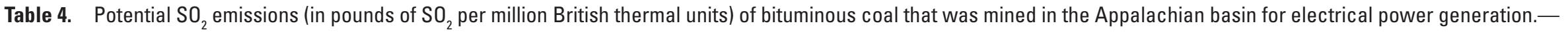
Continued

[Data are shown by county. Data for 1983 through 1995 were calculated from data given in tables 1-3; data for 1996 through 2005 are from the COALdat database. n/a, not available]

\begin{tabular}{|c|c|c|c|c|c|c|c|c|c|c|c|c|c|c|c|}
\hline State & County & 1983 & 1984 & 1985 & 1986 & 1987 & 1988 & 1989 & 1990 & 1991 & 1992 & 1993 & 1994 & 1995 & $\begin{array}{c}\text { Weighted average } \\
\text { (1983-1995) }\end{array}$ \\
\hline Ky. & Knox & 1.71 & 1.82 & 2.19 & 2.45 & 2.55 & 2.32 & 2.02 & 1.86 & 1.14 & 1.53 & 1.80 & 3.10 & 1.76 & 2.18 \\
\hline Ky. & Laurel & 2.53 & 2.84 & 2.47 & 2.53 & 2.39 & 2.07 & 1.93 & 1.82 & 1.43 & 2.35 & 3.12 & 0.00 & 2.93 & 2.43 \\
\hline Ky. & Lawrence & 2.53 & 2.79 & 1.43 & 2.30 & 1.82 & 1.88 & 1.90 & 2.41 & 1.98 & 1.83 & 1.83 & 2.07 & 2.44 & 2.07 \\
\hline Ky. & Lee & 0.00 & 0.00 & 3.29 & 3.40 & 1.71 & 1.73 & 2.58 & 3.85 & 0.00 & 0.00 & 3.04 & 0.00 & 1.92 & 3.10 \\
\hline Ky. & Leslie & 2.35 & 2.38 & 2.16 & 2.01 & 2.10 & 2.44 & 2.24 & 2.20 & 2.03 & 1.97 & 2.18 & 1.91 & 1.81 & 2.10 \\
\hline Ky. & Letcher & 1.39 & 1.31 & 1.40 & 1.26 & 1.20 & 1.23 & 1.31 & 1.32 & 1.43 & 1.49 & 1.63 & 1.69 & 1.71 & 1.43 \\
\hline Ky. & McCreary & 1.98 & 1.70 & 2.40 & 2.47 & 2.60 & 3.69 & 0.00 & 0.00 & 0.00 & 1.49 & 1.72 & 1.47 & 1.40 & 2.39 \\
\hline Ky. & Magoffin & 1.62 & 1.73 & 1.86 & 1.81 & 1.84 & 1.82 & 1.87 & 1.80 & 1.87 & 1.84 & 1.68 & 1.67 & 1.77 & 1.80 \\
\hline Ky. & Martin & 1.38 & 1.43 & 1.49 & 1.48 & 1.49 & 1.47 & 1.47 & 1.51 & 1.41 & 1.41 & 1.53 & 1.52 & 1.34 & 1.46 \\
\hline Ky. & Morgan & 2.25 & 3.74 & 2.12 & 2.18 & 1.41 & 2.22 & 2.45 & 2.32 & 0.00 & 0.00 & 0.00 & 2.63 & 0.00 & 1.97 \\
\hline Ky. & Owsley & 2.07 & 1.68 & 1.73 & 3.04 & 3.43 & 3.83 & 3.25 & 2.11 & 2.40 & 2.07 & 3.22 & 2.49 & 0.00 & 2.91 \\
\hline Ку. & Perry & 1.86 & 1.84 & 1.78 & 1.84 & 1.81 & 1.84 & 1.80 & 1.72 & 1.76 & 1.64 & 1.84 & 1.78 & 1.61 & 1.77 \\
\hline Kу. & Pike & 1.34 & 1.49 & 1.44 & 1.48 & 1.41 & 1.38 & 1.45 & 1.46 & 1.47 & 1.51 & 1.58 & 1.54 & 1.40 & 1.47 \\
\hline Ky. & Pulaski & 2.63 & 2.75 & 2.99 & 3.26 & 3.16 & 2.73 & 2.45 & 2.62 & 2.60 & 2.71 & 2.84 & 2.63 & 2.31 & 2.76 \\
\hline Ky. & Rockcastle & 2.19 & 0.00 & 1.78 & 0.00 & 0.00 & 0.00 & 0.00 & 0.00 & 0.00 & 0.00 & 0.89 & 0.00 & 0.00 & 2.09 \\
\hline Ky. & Wayne & 0.00 & 0.00 & 0.00 & 1.01 & 0.00 & 0.00 & 0.00 & 0.00 & 0.00 & 0.00 & 0.00 & 0.00 & 0.00 & 1.01 \\
\hline Ky. & Whitley & 2.06 & 1.93 & 1.88 & 1.70 & 1.69 & 1.75 & 1.83 & 2.00 & 1.88 & 2.05 & 1.94 & 1.97 & 1.59 & 1.86 \\
\hline Ку. & Wolfe & 2.04 & 3.99 & 4.43 & 4.43 & 4.87 & 4.17 & 2.48 & 0.00 & 1.96 & 2.01 & 2.09 & 2.10 & 2.08 & 3.72 \\
\hline Md. & Allegany & 2.99 & 3.08 & 2.29 & 2.57 & 2.71 & 2.68 & 3.02 & 2.93 & 2.79 & 2.70 & 2.60 & 2.39 & 2.55 & 2.79 \\
\hline Md. & Garrett & 2.67 & 2.68 & 2.68 & 2.47 & 2.59 & 2.47 & 2.52 & 2.48 & 2.46 & 2.67 & 2.66 & 2.54 & 2.35 & 2.54 \\
\hline Ohio & Athens & 5.19 & 0.00 & 0.00 & 0.00 & 2.93 & 4.62 & 4.73 & 3.98 & 4.27 & 0.00 & 0.00 & 0.00 & 0.00 & 4.03 \\
\hline Ohio & Belmont & 5.02 & 4.98 & 4.82 & 4.86 & 4.86 & 4.87 & 5.29 & 5.78 & 6.02 & 6.01 & 6.03 & 6.42 & 6.33 & 5.41 \\
\hline Ohio & Carroll & 3.29 & 4.04 & 4.95 & 5.24 & 4.45 & 4.93 & 4.13 & 3.65 & 4.57 & 4.10 & 4.26 & 4.59 & 4.96 & 4.44 \\
\hline Ohio & Columbiana & 3.69 & 4.14 & 4.25 & 3.92 & 3.76 & 4.21 & 4.44 & 4.11 & 4.44 & 3.53 & 3.62 & 3.67 & 3.63 & 3.99 \\
\hline Ohio & Coshocton & 5.27 & 5.37 & 5.34 & 5.06 & 5.35 & 5.40 & 5.40 & 5.43 & 5.35 & 5.36 & 5.32 & 4.92 & 4.22 & 5.22 \\
\hline Ohio & Gallia & 5.92 & 5.38 & 5.30 & 6.02 & 5.40 & 0.00 & 0.00 & 0.00 & 0.00 & 0.00 & 6.04 & 5.49 & 5.35 & 5.58 \\
\hline Ohio & Guernsey & 0.00 & 8.27 & 0.00 & 0.00 & 0.00 & 0.00 & 7.31 & 7.46 & 3.16 & 3.14 & 3.61 & 4.64 & 5.19 & 5.84 \\
\hline Ohio & Harrison & 4.25 & 4.40 & 4.27 & 4.02 & 3.86 & 4.02 & 4.12 & 4.08 & 5.16 & 5.16 & 4.41 & 5.47 & 6.34 & 4.63 \\
\hline Ohio & Hocking & 5.94 & 6.32 & 5.93 & 5.91 & 5.67 & 5.78 & 6.23 & 6.34 & 6.30 & 6.14 & 6.22 & 6.59 & 0.00 & 6.07 \\
\hline Ohio & Holmes & 0.00 & 0.00 & 0.00 & 0.00 & 0.00 & 6.01 & 6.45 & 6.57 & 5.91 & 5.78 & 6.08 & 6.32 & 5.79 & 6.14 \\
\hline
\end{tabular}


Table 4. Potential $\mathrm{SO}_{2}$ emissions (in pounds of $\mathrm{SO}_{2}$ per million British thermal units) of bituminous coal that was mined in the Appalachian basin for electrical power generation.-Continued

[Data are shown by county. Data for 1983 through 1995 were calculated from data given in tables 1-3; data for 1996 through 2005 are from the COALdat database. n/a, not available]

\begin{tabular}{|c|c|c|c|c|c|c|c|c|c|c|c|c|}
\hline State & County & 1996 & 1997 & 1998 & 1999 & 2000 & 2001 & 2002 & 2003 & 2004 & 2005 & $\begin{array}{c}\text { Weighted average } \\
\text { (1996-2005) }\end{array}$ \\
\hline Ky. & Knox & 0.00 & 0.00 & 6.11 & 0.00 & 0.00 & 0.00 & 1.56 & 1.76 & 2.79 & 2.83 & 2.80 \\
\hline Ky. & Laurel & 1.40 & 0.00 & 2.08 & 2.67 & 1.38 & 1.20 & 0.00 & 1.83 & 1.33 & 0.00 & 1.58 \\
\hline Ky. & Lawrence & 1.65 & 1.48 & 1.58 & 1.87 & 2.08 & 2.00 & 1.76 & 2.39 & 2.40 & 2.01 & 2.13 \\
\hline Ky. & Lee & 0.00 & 0.00 & 0.00 & 0.00 & 0.00 & 0.00 & 1.53 & 0.00 & 0.00 & 0.00 & 1.53 \\
\hline Ky. & Leslie & 1.71 & 1.60 & 1.63 & 1.76 & 1.56 & 1.77 & 2.00 & 2.10 & 2.06 & 1.95 & 1.70 \\
\hline Ky. & Letcher & 1.79 & 1.86 & 1.88 & 2.18 & 1.76 & 1.87 & 1.73 & 1.86 & 1.89 & 1.96 & 1.88 \\
\hline Ky. & McCreary & 1.42 & 0.00 & 0.00 & 0.00 & 0.00 & 0.00 & 0.00 & 0.00 & 0.00 & 0.00 & 1.42 \\
\hline Ky. & Magoffin & 1.57 & 2.82 & 1.68 & 1.28 & 1.09 & 1.11 & 1.11 & 1.14 & 1.43 & 1.63 & 1.59 \\
\hline Ky. & Martin & 1.42 & 1.49 & 1.52 & 1.43 & 1.50 & 1.49 & 1.38 & 1.42 & 1.54 & 1.66 & 1.48 \\
\hline Ку. & Morgan & 0.00 & 0.00 & 1.14 & 1.14 & 1.46 & 2.08 & 0.00 & 0.00 & 0.00 & 0.00 & 1.78 \\
\hline Ky. & Owsley & 0.00 & 0.00 & 1.95 & 2.70 & 0.00 & 0.00 & 1.33 & 2.49 & 2.64 & 2.54 & 2.42 \\
\hline Ky. & Perry & 1.62 & 1.62 & 1.69 & 1.62 & 1.57 & 1.68 & 1.61 & 1.63 & 1.63 & 1.67 & 1.64 \\
\hline Ky. & Pike & 1.46 & 1.58 & 1.53 & 1.53 & 1.47 & 1.61 & 1.64 & 1.64 & 1.63 & 1.77 & 1.59 \\
\hline Ky. & Pulaski & 0.00 & 0.00 & 2.19 & 2.11 & 2.03 & 2.37 & 2.18 & 2.08 & 2.13 & 0.00 & 2.13 \\
\hline Ky. & Rockcastle & 0.00 & 0.00 & 0.00 & 0.00 & 0.00 & 0.00 & 0.00 & 0.00 & 0.00 & 1.32 & 1.32 \\
\hline Ky. & Wayne & $\mathrm{n} / \mathrm{a}$ & $\mathrm{n} / \mathrm{a}$ & $\mathrm{n} / \mathrm{a}$ & $\mathrm{n} / \mathrm{a}$ & $\mathrm{n} / \mathrm{a}$ & $\mathrm{n} / \mathrm{a}$ & $\mathrm{n} / \mathrm{a}$ & $\mathrm{n} / \mathrm{a}$ & $\mathrm{n} / \mathrm{a}$ & $\mathrm{n} / \mathrm{a}$ & No production \\
\hline Ky. & Whitley & 1.64 & 1.64 & 1.84 & 1.56 & 1.86 & 1.59 & 1.55 & 1.72 & 1.78 & 1.91 & 1.74 \\
\hline Ky. & Wolfe & 1.64 & 1.60 & 1.73 & 1.76 & 0.00 & 0.00 & 0.00 & 0.00 & 0.00 & 0.00 & 1.65 \\
\hline Md. & Allegany & 2.34 & 2.17 & 2.43 & 2.23 & 1.67 & 1.59 & 2.75 & 2.73 & 2.84 & 3.01 & 2.69 \\
\hline Md. & Garrett & 2.48 & 2.67 & 2.71 & 3.04 & 2.94 & 2.75 & 2.74 & 2.23 & 2.68 & 3.13 & 2.74 \\
\hline Ohio & Athens & 0.00 & 0.00 & 0.00 & 0.00 & 0.00 & 0.00 & 3.50 & 0.00 & 3.23 & 2.86 & 3.06 \\
\hline Ohio & Belmont & 6.27 & 6.12 & 6.14 & 6.26 & 6.12 & 6.31 & 6.31 & 6.45 & 6.51 & 6.61 & 6.30 \\
\hline Ohio & Carroll & 0.00 & 4.12 & 0.00 & 6.29 & 0.00 & 0.00 & 2.55 & 2.66 & 3.14 & 2.08 & 2.82 \\
\hline Ohio & Columbiana & 3.45 & 3.91 & 3.64 & 3.81 & 3.54 & 3.65 & 4.29 & 4.74 & 4.48 & 4.25 & 3.89 \\
\hline Ohio & Coshocton & 4.50 & 4.55 & 4.52 & 4.50 & 3.93 & 3.68 & 4.21 & 3.87 & 3.82 & 3.83 & 4.13 \\
\hline Ohio & Gallia & 5.11 & 5.11 & 4.90 & 5.07 & 5.18 & 4.94 & 6.00 & 6.20 & 6.00 & 5.80 & 5.89 \\
\hline Ohio & Guernsey & 8.43 & 7.52 & 7.72 & 3.71 & 5.24 & 5.48 & 0.00 & 0.00 & 0.00 & 0.00 & 7.89 \\
\hline Ohio & Harrison & 5.10 & 5.10 & 4.82 & 4.25 & 4.28 & 4.19 & 4.27 & 4.79 & 4.72 & 4.61 & 4.62 \\
\hline Ohio & Hocking & $\mathrm{n} / \mathrm{a}$ & $\mathrm{n} / \mathrm{a}$ & $\mathrm{n} / \mathrm{a}$ & $\mathrm{n} / \mathrm{a}$ & $\mathrm{n} / \mathrm{a}$ & $\mathrm{n} / \mathrm{a}$ & $\mathrm{n} / \mathrm{a}$ & $\mathrm{n} / \mathrm{a}$ & $\mathrm{n} / \mathrm{a}$ & $\mathrm{n} / \mathrm{a}$ & No production \\
\hline Ohio & Holmes & 5.85 & 5.97 & 6.17 & 6.22 & 6.57 & 6.64 & 7.28 & 0.00 & 0.00 & 0.00 & 6.28 \\
\hline
\end{tabular}




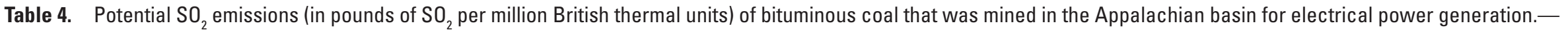
Continued

[Data are shown by county. Data for 1983 through 1995 were calculated from data given in tables 1-3; data for 1996 through 2005 are from the COALdat database. n/a, not available]

\begin{tabular}{|c|c|c|c|c|c|c|c|c|c|c|c|c|c|c|c|}
\hline State & County & 1983 & 1984 & 1985 & 1986 & 1987 & 1988 & 1989 & 1990 & 1991 & 1992 & 1993 & 1994 & 1995 & $\begin{array}{c}\text { Weighted average } \\
\text { (1983-1995) }\end{array}$ \\
\hline Ohio & Jackson & 4.14 & 5.29 & 5.40 & 4.09 & 5.22 & 5.66 & 5.66 & 5.91 & 5.83 & 6.25 & 6.20 & 6.07 & 6.69 & 5.76 \\
\hline Ohio & Jefferson & 4.70 & 5.13 & 5.03 & 4.89 & 4.47 & 4.51 & 4.80 & 5.03 & 4.94 & 5.08 & 5.16 & 5.41 & 4.52 & 4.90 \\
\hline Ohio & Lawrence & 6.10 & 5.83 & 5.37 & 5.74 & 4.33 & 5.24 & 4.31 & 4.45 & 4.79 & 4.46 & 4.40 & 4.86 & 4.80 & 4.73 \\
\hline Ohio & Mahoning & 5.04 & 4.59 & 4.74 & 5.23 & 5.04 & 5.92 & 5.57 & 4.86 & 5.22 & 6.56 & 5.26 & 6.01 & 5.07 & 5.22 \\
\hline Ohio & Meigs & 6.25 & 6.11 & 5.96 & 6.03 & 6.12 & 6.08 & 5.83 & 6.12 & 6.11 & 5.93 & 5.74 & 5.37 & 5.31 & 5.93 \\
\hline Ohio & Monroe & 6.25 & 6.74 & 6.86 & 6.84 & 6.68 & 6.57 & 6.64 & 6.86 & 6.73 & 6.37 & 5.44 & 6.17 & 6.03 & 6.60 \\
\hline Ohio & Morgan & 7.66 & 7.50 & 7.54 & 0.00 & 0.00 & 0.00 & 4.33 & 0.00 & 0.00 & 0.00 & 0.00 & 0.00 & 0.00 & 7.29 \\
\hline Ohio & Muskingum & 7.52 & 7.30 & 7.45 & 7.52 & 7.11 & 7.29 & 7.36 & 7.20 & 7.40 & 7.39 & 7.90 & 7.87 & 7.93 & 7.43 \\
\hline Ohio & Noble & 7.68 & 7.51 & 7.46 & 7.69 & 7.98 & 8.06 & 8.10 & 8.04 & 8.28 & 7.72 & 8.01 & 8.22 & 8.39 & 8.00 \\
\hline Ohio & Perry & 4.38 & 4.39 & 3.79 & 3.78 & 3.63 & 4.14 & 4.40 & 4.10 & 5.26 & 5.07 & 4.83 & 5.93 & 5.67 & 4.21 \\
\hline Ohio & Pike & 0.00 & 0.00 & 0.00 & 0.00 & 0.00 & 0.00 & 0.00 & 0.00 & 0.00 & 0.00 & 0.00 & 0.00 & 4.35 & 4.35 \\
\hline Ohio & Scioto & 0.00 & 1.86 & 0.00 & 0.00 & 0.00 & 0.00 & 0.00 & 0.00 & 0.00 & 0.00 & 0.00 & 0.00 & 0.00 & 1.86 \\
\hline Ohio & Stark & 5.12 & 7.07 & 6.09 & 6.28 & 5.37 & 5.69 & 6.63 & 5.63 & 6.70 & 4.57 & 4.51 & 0.00 & 0.00 & 5.95 \\
\hline Ohio & Tuscarawas & 6.17 & 5.23 & 5.39 & 4.76 & 5.00 & 5.30 & 5.15 & 5.04 & 5.68 & 5.43 & 5.75 & 5.81 & 6.09 & 5.45 \\
\hline Ohio & Vinton & 5.70 & 6.28 & 6.13 & 5.80 & 5.43 & 5.14 & 5.63 & 5.68 & 5.68 & 5.72 & 5.71 & 5.52 & 5.33 & 5.65 \\
\hline Ohio & Washington & 0.00 & 0.00 & 0.00 & 0.00 & 0.00 & 0.00 & 0.00 & 0.00 & 0.00 & 0.00 & 0.00 & 0.00 & 0.00 & 0.00 \\
\hline $\mathrm{Pa}$. & Allegheny & 1.96 & 1.82 & 2.11 & 2.27 & 2.11 & 2.13 & 2.22 & 2.33 & 2.48 & 2.66 & 4.53 & 3.54 & 0.00 & 2.25 \\
\hline Pa. & Armstrong & 2.92 & 2.66 & 2.53 & 2.53 & 2.48 & 2.67 & 2.72 & 2.78 & 2.81 & 2.76 & 3.08 & 2.92 & 2.91 & 2.74 \\
\hline Pa. & Beaver & 0.00 & 0.00 & 0.00 & 5.43 & 4.96 & 4.37 & 3.22 & 3.28 & 4.66 & 3.53 & 2.89 & 2.70 & 0.00 & 3.97 \\
\hline Pa. & Bedford & 3.38 & 3.21 & 3.07 & 3.02 & 2.65 & 2.91 & 2.34 & 2.90 & 3.68 & 2.47 & 2.56 & 0.00 & 0.00 & 3.01 \\
\hline $\mathrm{Pa}$. & Blair & 0.00 & 0.00 & 0.00 & 0.00 & 0.00 & 0.00 & 0.00 & 0.00 & 0.00 & 2.79 & 2.50 & 0.00 & 0.00 & 2.76 \\
\hline Pa. & Bradford & 1.57 & 0.00 & 0.00 & 0.00 & 0.00 & 0.00 & 0.00 & 0.00 & 0.00 & 0.00 & 0.00 & 0.00 & 0.00 & 1.57 \\
\hline Pa. & Butler & 2.96 & 3.03 & 3.04 & 3.50 & 3.91 & 4.22 & 3.26 & 2.96 & 3.04 & 3.62 & 2.98 & 3.18 & 2.84 & 3.36 \\
\hline Pa. & Cambria & 2.48 & 2.52 & 2.35 & 2.39 & 2.32 & 2.78 & 2.68 & 2.79 & 2.76 & 3.07 & 3.00 & 2.96 & 2.88 & 2.64 \\
\hline Pa. & Cameron & 0.00 & 0.00 & 0.00 & 0.00 & 1.00 & 0.00 & 0.00 & 0.00 & 0.00 & 0.00 & 0.00 & 0.00 & 0.00 & 1.00 \\
\hline Pa. & Centre & 2.89 & 3.09 & 2.84 & 3.12 & 2.95 & 2.65 & 2.82 & 2.69 & 2.80 & 2.96 & 2.86 & 2.77 & 2.66 & 2.89 \\
\hline Pa. & Clarion & 3.76 & 3.85 & 3.69 & 3.54 & 3.15 & 3.39 & 3.49 & 3.55 & 3.50 & 3.59 & 3.23 & 2.78 & 2.52 & 3.48 \\
\hline Pa. & Clearfield & 2.91 & 3.00 & 2.91 & 2.90 & 2.98 & 2.96 & 2.94 & 3.16 & 3.09 & 3.11 & 3.01 & 2.97 & 2.87 & 2.98 \\
\hline Pa. & Clinton & 2.27 & 0.00 & 1.54 & 1.14 & 2.75 & 3.32 & 1.24 & 1.75 & 1.27 & 1.34 & 0.00 & 2.65 & 0.00 & 1.73 \\
\hline Pa. & Elk & 2.81 & 3.06 & 2.56 & 2.87 & 2.66 & 3.19 & 2.91 & 2.88 & 2.70 & 2.70 & 3.04 & 2.62 & 2.32 & 2.79 \\
\hline
\end{tabular}


Table 4. Potential $\mathrm{SO}_{2}$ emissions (in pounds of $\mathrm{SO}_{2}$ per million British thermal units) of bituminous coal that was mined in the Appalachian basin for electrical power generation.-Continued

[Data are shown by county. Data for 1983 through 1995 were calculated from data given in tables 1-3; data for 1996 through 2005 are from the COALdat database. n/a, not available]

\begin{tabular}{|c|c|c|c|c|c|c|c|c|c|c|c|c|}
\hline State & County & 1996 & 1997 & 1998 & 1999 & 2000 & 2001 & 2002 & 2003 & 2004 & 2005 & $\begin{array}{c}\text { Weighted average } \\
(1996-2005)\end{array}$ \\
\hline Ohio & Jackson & 6.65 & 6.20 & 6.50 & 6.45 & 5.57 & 5.26 & 5.58 & 5.89 & 5.97 & 6.60 & 6.10 \\
\hline Ohio & Jefferson & 6.04 & 5.19 & 4.13 & 3.84 & 4.18 & 4.24 & 3.94 & 3.97 & 3.72 & 3.92 & 4.17 \\
\hline Ohio & Lawrence & 0.00 & 4.91 & 0.00 & 0.00 & 0.00 & 0.00 & 0.00 & 0.00 & 0.00 & 0.00 & 4.91 \\
\hline Ohio & Mahoning & $\mathrm{n} / \mathrm{a}$ & $\mathrm{n} / \mathrm{a}$ & $\mathrm{n} / \mathrm{a}$ & $\mathrm{n} / \mathrm{a}$ & $\mathrm{n} / \mathrm{a}$ & $\mathrm{n} / \mathrm{a}$ & $\mathrm{n} / \mathrm{a}$ & $\mathrm{n} / \mathrm{a}$ & $\mathrm{n} / \mathrm{a}$ & $\mathrm{n} / \mathrm{a}$ & No production \\
\hline Ohio & Meigs & 5.64 & 6.20 & 6.29 & 6.29 & 6.26 & 6.40 & 6.47 & 0.00 & 0.00 & 0.00 & 6.16 \\
\hline Ohio & Monroe & 7.06 & 7.05 & 7.22 & 7.17 & 6.33 & 5.94 & 6.74 & 6.64 & 7.02 & 6.95 & 6.96 \\
\hline Ohio & Morgan & $\mathrm{n} / \mathrm{a}$ & $\mathrm{n} / \mathrm{a}$ & $\mathrm{n} / \mathrm{a}$ & $\mathrm{n} / \mathrm{a}$ & $\mathrm{n} / \mathrm{a}$ & $\mathrm{n} / \mathrm{a}$ & $\mathrm{n} / \mathrm{a}$ & $\mathrm{n} / \mathrm{a}$ & $\mathrm{n} / \mathrm{a}$ & $\mathrm{n} / \mathrm{a}$ & No production \\
\hline Ohio & Muskingum & 8.54 & 7.53 & 7.74 & 7.95 & 5.48 & 5.21 & 5.18 & 0.00 & 0.00 & 6.52 & 6.30 \\
\hline Ohio & Noble & 8.59 & 8.73 & 8.81 & 8.03 & 5.20 & 4.78 & 4.27 & 3.41 & 4.47 & 7.10 & 6.29 \\
\hline Ohio & Perry & 5.18 & 5.32 & 4.94 & 4.91 & 5.80 & 6.17 & 6.65 & 5.73 & 6.53 & 6.47 & 5.80 \\
\hline Ohio & Pike & $\mathrm{n} / \mathrm{a}$ & $\mathrm{n} / \mathrm{a}$ & $\mathrm{n} / \mathrm{a}$ & $\mathrm{n} / \mathrm{a}$ & $\mathrm{n} / \mathrm{a}$ & $\mathrm{n} / \mathrm{a}$ & $\mathrm{n} / \mathrm{a}$ & $\mathrm{n} / \mathrm{a}$ & $\mathrm{n} / \mathrm{a}$ & $\mathrm{n} / \mathrm{a}$ & No production \\
\hline Ohio & Scioto & 0.00 & 0.00 & 0.00 & 0.00 & 0.00 & 0.00 & 0.00 & 0.00 & 3.08 & 0.00 & 3.08 \\
\hline Ohio & Stark & 6.56 & 6.18 & 5.49 & 5.03 & 3.72 & 3.07 & 0.00 & 0.00 & 0.00 & 0.00 & 5.48 \\
\hline Ohio & Tuscarawas & 5.89 & 5.04 & 6.02 & 5.82 & 6.40 & 6.61 & 6.73 & 6.94 & 5.09 & 0.00 & 6.10 \\
\hline Ohio & Vinton & 4.74 & 5.26 & 5.20 & 5.30 & 5.13 & 5.58 & 5.67 & 5.29 & 5.58 & 5.22 & 5.26 \\
\hline Ohio & Washington & 0.00 & 0.00 & 0.00 & 0.00 & 0.00 & 0.00 & 0.00 & 4.00 & 4.01 & 4.14 & 4.05 \\
\hline $\mathrm{Pa}$. & Allegheny & 3.67 & 2.08 & 0.00 & 2.40 & 3.80 & 3.89 & 1.16 & 0.00 & 0.00 & 2.99 & 2.56 \\
\hline Pa. & Armstrong & 3.17 & 3.48 & 3.54 & 3.13 & 3.55 & 3.25 & 3.62 & 3.90 & 3.88 & 3.62 & 3.60 \\
\hline Pa. & Beaver & 0.00 & 0.00 & 0.00 & 1.48 & 3.55 & 0.00 & 2.98 & 2.77 & 4.30 & 3.44 & 3.64 \\
\hline Pa. & Bedford & 0.00 & 0.00 & 0.00 & 1.76 & 1.55 & 0.00 & 0.00 & 2.35 & 2.28 & 0.00 & 1.76 \\
\hline $\mathrm{Pa}$. & Blair & 5.09 & 4.09 & 0.00 & 0.00 & 0.00 & 0.00 & 0.00 & 0.00 & 0.00 & 0.00 & 4.58 \\
\hline Pa. & Bradford & $\mathrm{n} / \mathrm{a}$ & $\mathrm{n} / \mathrm{a}$ & $\mathrm{n} / \mathrm{a}$ & $\mathrm{n} / \mathrm{a}$ & $\mathrm{n} / \mathrm{a}$ & $\mathrm{n} / \mathrm{a}$ & $\mathrm{n} / \mathrm{a}$ & $\mathrm{n} / \mathrm{a}$ & $\mathrm{n} / \mathrm{a}$ & $\mathrm{n} / \mathrm{a}$ & No production \\
\hline Pa. & Butler & 3.28 & 3.10 & 3.42 & 3.32 & 2.64 & 2.67 & 2.91 & 1.75 & 3.50 & 4.71 & 3.15 \\
\hline Pa. & Cambria & 2.88 & 3.26 & 3.47 & 3.56 & 3.26 & 4.03 & 5.28 & 6.59 & 6.84 & 7.94 & 5.71 \\
\hline Pa. & Cameron & $\mathrm{n} / \mathrm{a}$ & $\mathrm{n} / \mathrm{a}$ & $\mathrm{n} / \mathrm{a}$ & $\mathrm{n} / \mathrm{a}$ & $\mathrm{n} / \mathrm{a}$ & $\mathrm{n} / \mathrm{a}$ & $\mathrm{n} / \mathrm{a}$ & $\mathrm{n} / \mathrm{a}$ & $\mathrm{n} / \mathrm{a}$ & $\mathrm{n} / \mathrm{a}$ & No production \\
\hline Pa. & Centre & 2.30 & 1.93 & 1.83 & 0.00 & 0.00 & 0.00 & 0.00 & 3.63 & 0.00 & 0.00 & 2.42 \\
\hline Pa. & Clarion & 2.73 & 2.91 & 2.56 & 2.73 & 1.97 & 1.94 & 3.39 & 1.82 & 2.71 & 3.48 & 2.71 \\
\hline Pa. & Clearfield & 3.06 & 3.09 & 2.91 & 2.91 & 2.81 & 2.93 & 2.90 & 2.86 & 3.14 & 2.93 & 2.97 \\
\hline Pa. & Clinton & $\mathrm{n} / \mathrm{a}$ & $\mathrm{n} / \mathrm{a}$ & $\mathrm{n} / \mathrm{a}$ & $\mathrm{n} / \mathrm{a}$ & $\mathrm{n} / \mathrm{a}$ & $\mathrm{n} / \mathrm{a}$ & $\mathrm{n} / \mathrm{a}$ & $\mathrm{n} / \mathrm{a}$ & $\mathrm{n} / \mathrm{a}$ & $\mathrm{n} / \mathrm{a}$ & No production \\
\hline Pa. & Elk & 2.13 & 2.78 & 3.22 & 2.58 & 3.04 & 3.58 & 3.10 & 2.88 & 3.48 & 3.38 & 3.18 \\
\hline
\end{tabular}




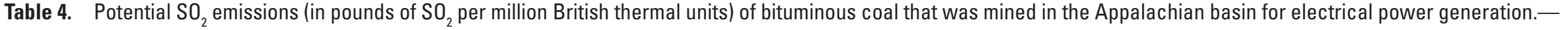
Continued

[Data are shown by county. Data for 1983 through 1995 were calculated from data given in tables 1-3; data for 1996 through 2005 are from the COALdat database. n/a, not available]

\begin{tabular}{|c|c|c|c|c|c|c|c|c|c|c|c|c|c|c|c|}
\hline State & County & 1983 & 1984 & 1985 & 1986 & 1987 & 1988 & 1989 & 1990 & 1991 & 1992 & 1993 & 1994 & 1995 & $\begin{array}{c}\text { Weighted average } \\
\text { (1983-1995) }\end{array}$ \\
\hline Pa. & Fayette & 3.19 & 3.48 & 3.22 & 3.12 & 2.89 & 2.94 & 2.80 & 2.96 & 2.54 & 2.70 & 2.76 & 2.28 & 2.97 & 2.99 \\
\hline $\mathrm{Pa}$. & Fulton & 2.22 & 3.10 & 3.02 & 3.37 & 3.05 & 3.19 & 3.29 & 3.70 & 3.25 & 3.79 & 3.44 & 0.00 & 2.50 & 3.19 \\
\hline $\mathrm{Pa}$. & Greene & 2.99 & 3.20 & 2.76 & 2.53 & 2.47 & 2.45 & 2.40 & 2.67 & 2.73 & 2.73 & 3.02 & 2.79 & 2.60 & 2.73 \\
\hline Pa. & Huntingdon & 0.00 & 0.00 & 0.00 & 0.00 & 0.00 & 0.00 & 0.00 & 0.00 & 2.82 & 2.90 & 2.53 & 4.24 & 0.00 & 3.43 \\
\hline Pa. & Indiana & 3.19 & 3.20 & 3.29 & 3.34 & 3.21 & 3.18 & 3.08 & 3.20 & 3.22 & 3.08 & 2.91 & 3.09 & 2.82 & 3.17 \\
\hline $\mathrm{Pa}$. & Jefferson & 2.77 & 2.69 & 2.69 & 2.67 & 2.85 & 2.90 & 2.72 & 2.60 & 2.61 & 2.71 & 2.84 & 2.47 & 2.46 & 2.70 \\
\hline $\mathrm{Pa}$. & Lawrence & 3.32 & 4.44 & 4.33 & 4.18 & 3.04 & 3.54 & 3.95 & 4.09 & 3.38 & 4.51 & 4.20 & 0.00 & 2.36 & 3.78 \\
\hline $\mathrm{Pa}$. & Lycoming & 1.44 & 1.47 & 1.30 & 1.35 & 1.44 & 1.53 & 1.57 & 1.80 & 1.69 & 1.78 & 1.64 & 1.71 & 1.70 & 1.59 \\
\hline $\mathrm{Pa}$. & Mercer & 3.67 & 2.42 & 2.42 & 2.54 & 2.14 & 2.46 & 2.48 & 2.62 & 2.65 & 3.06 & 2.40 & 4.71 & 0.00 & 2.72 \\
\hline $\mathrm{Pa}$. & Somerset & 2.38 & 2.02 & 2.05 & 2.26 & 2.45 & 2.57 & 2.49 & 2.69 & 2.72 & 3.11 & 3.21 & 3.04 & 3.37 & 2.79 \\
\hline $\mathrm{Pa}$. & Tioga & 2.03 & 2.04 & 1.82 & 1.54 & 1.79 & 2.40 & 2.27 & 1.89 & 0.00 & 0.00 & 0.00 & 0.00 & 0.00 & 1.96 \\
\hline $\mathrm{Pa}$. & Venango & 5.30 & 5.21 & 4.86 & 3.87 & 3.81 & 3.63 & 3.43 & 3.66 & 3.22 & 2.60 & 0.00 & 0.00 & 0.00 & 4.11 \\
\hline Pa. & Washington & 2.87 & 2.76 & 2.94 & 2.74 & 2.38 & 2.62 & 3.17 & 3.65 & 3.99 & 3.52 & 4.12 & 3.93 & 2.96 & 3.33 \\
\hline Pa. & Westmoreland & 2.55 & 2.03 & 1.88 & 2.25 & 3.09 & 3.45 & 3.05 & 3.08 & 3.34 & 3.38 & 3.54 & 3.58 & 3.42 & 3.13 \\
\hline Tenn. & Anderson & 2.84 & 2.55 & 2.36 & 2.38 & 2.66 & 3.06 & 3.40 & 3.54 & 3.29 & 3.74 & 2.47 & 2.43 & 1.05 & 2.97 \\
\hline Tenn. & Bledsoe & 0.00 & 2.02 & 1.87 & 2.05 & 1.02 & 0.00 & 0.00 & 0.00 & 0.00 & 0.00 & 0.00 & 0.00 & 0.00 & 1.88 \\
\hline Tenn. & Campbell & 2.98 & 2.85 & 2.73 & 2.66 & 2.52 & 2.79 & 1.96 & 2.09 & 1.89 & 2.06 & 2.21 & 1.98 & 1.66 & 2.37 \\
\hline Tenn. & Claiborne & 1.36 & 1.39 & 1.53 & 1.61 & 1.62 & 1.62 & 1.65 & 1.75 & 2.42 & 1.76 & 0.00 & 0.00 & 2.31 & 1.58 \\
\hline Tenn. & Cumberland & 2.34 & 2.02 & 1.27 & 1.39 & 1.54 & 1.36 & 0.00 & 0.00 & 0.00 & 0.00 & 0.00 & 0.00 & 0.00 & 1.77 \\
\hline Tenn. & Fentress & 3.13 & 4.17 & 4.38 & 3.20 & 2.90 & 5.29 & 2.25 & 3.20 & 0.00 & 0.00 & 0.00 & 0.00 & 0.00 & 3.60 \\
\hline Tenn. & Grundy & 1.43 & 1.39 & 1.52 & 1.43 & 1.33 & 1.27 & 1.22 & 1.54 & 1.48 & 0.00 & 0.00 & 0.00 & 0.00 & 1.43 \\
\hline Tenn. & Marion & 0.00 & 0.00 & 2.73 & 1.34 & 2.41 & 1.44 & 1.15 & 1.15 & 0.00 & 0.00 & 0.00 & 0.00 & 0.00 & 1.30 \\
\hline Tenn. & Morgan & 1.89 & 1.45 & 2.35 & 1.88 & 2.22 & 2.17 & 2.19 & 3.12 & 2.74 & 2.84 & 3.50 & 3.68 & 2.09 & 2.33 \\
\hline Tenn. & Rhea & 1.83 & 3.15 & 2.19 & 2.47 & 1.85 & 0.00 & 0.00 & 0.00 & 0.00 & 0.00 & 0.00 & 0.00 & 0.00 & 2.61 \\
\hline Tenn. & Scott & 3.09 & 3.77 & 2.88 & 2.95 & 2.30 & 2.05 & 2.55 & 2.38 & 2.30 & 2.50 & 2.41 & 2.22 & 1.95 & 2.46 \\
\hline Tenn. & Sequatchie & 4.78 & 5.14 & 5.68 & 1.79 & 1.18 & 1.27 & 1.35 & 1.28 & 1.62 & 1.29 & 1.47 & 1.40 & 1.38 & 1.50 \\
\hline Tenn. & Van Buren & 3.15 & 0.00 & 0.00 & 0.00 & 0.00 & 0.00 & 0.00 & 0.00 & 0.00 & 0.00 & 0.00 & 0.00 & 0.00 & 3.15 \\
\hline Va. & Buchanan & 1.65 & 1.60 & 1.68 & 1.73 & 1.63 & 1.62 & 1.60 & 1.59 & 1.46 & 1.48 & 1.36 & 1.31 & 1.26 & 1.55 \\
\hline Va. & Dickenson & 1.66 & 1.64 & 1.90 & 1.94 & 1.98 & 2.17 & 2.02 & 1.83 & 1.81 & 1.54 & 1.46 & 1.43 & 1.59 & 1.70 \\
\hline Va. & Lee & 1.42 & 1.36 & 1.34 & 1.41 & 1.47 & 1.82 & 1.93 & 1.81 & 1.80 & 1.79 & 1.99 & 1.82 & 1.34 & 1.64 \\
\hline
\end{tabular}


Table 4. Potential $\mathrm{SO}_{2}$ emissions (in pounds of $\mathrm{SO}_{2}$ per million British thermal units) of bituminous coal that was mined in the Appalachian basin for electrical power generation.-Continued

[Data are shown by county. Data for 1983 through 1995 were calculated from data given in tables 1-3; data for 1996 through 2005 are from the COALdat database. n/a, not available]

\begin{tabular}{|c|c|c|c|c|c|c|c|c|c|c|c|c|}
\hline State & County & 1996 & 1997 & 1998 & 1999 & 2000 & 2001 & 2002 & 2003 & 2004 & 2005 & $\begin{array}{c}\text { Weighted average } \\
\text { (1996-2005) }\end{array}$ \\
\hline $\mathrm{Pa}$. & Fayette & 2.98 & 2.65 & 3.29 & 2.54 & 2.46 & 2.54 & 1.78 & 1.81 & 3.00 & 4.74 & 3.00 \\
\hline $\mathrm{Pa}$. & Fulton & 3.39 & 0.00 & 0.00 & 0.00 & 0.00 & 0.00 & 0.00 & 0.00 & 0.00 & 0.00 & 3.39 \\
\hline $\mathrm{Pa}$. & Greene & 2.78 & 2.78 & 2.75 & 2.94 & 2.90 & 2.90 & 3.08 & 3.37 & 3.20 & 3.02 & 2.96 \\
\hline $\mathrm{Pa}$. & Huntingdon & $\mathrm{n} / \mathrm{a}$ & $\mathrm{n} / \mathrm{a}$ & $\mathrm{n} / \mathrm{a}$ & $\mathrm{n} / \mathrm{a}$ & $\mathrm{n} / \mathrm{a}$ & $\mathrm{n} / \mathrm{a}$ & $\mathrm{n} / \mathrm{a}$ & $\mathrm{n} / \mathrm{a}$ & $\mathrm{n} / \mathrm{a}$ & $\mathrm{n} / \mathrm{a}$ & No production \\
\hline $\mathrm{Pa}$. & Indiana & 2.99 & 2.96 & 2.98 & 3.50 & 3.75 & 3.73 & 4.61 & 4.54 & 4.28 & 4.36 & 3.62 \\
\hline $\mathrm{Pa}$. & Jefferson & 2.77 & 2.79 & 2.72 & 2.77 & 2.82 & 2.46 & 2.83 & 2.45 & 3.65 & 3.13 & 2.81 \\
\hline $\mathrm{Pa}$. & Lawrence & 1.99 & 0.00 & 0.00 & 0.00 & 5.25 & 0.00 & 0.00 & 9.14 & 0.00 & 6.96 & 4.69 \\
\hline $\mathrm{Pa}$. & Lycoming & 1.30 & 1.50 & 1.29 & 1.32 & 1.14 & 0.00 & 1.65 & 1.57 & 1.94 & 1.69 & 1.44 \\
\hline $\mathrm{Pa}$. & Mercer & 0.00 & 0.00 & 0.00 & 0.00 & 0.00 & 0.00 & 0.00 & 11.64 & 10.11 & 0.00 & 10.90 \\
\hline $\mathrm{Pa}$. & Somerset & 3.06 & 3.41 & 3.45 & 3.14 & 3.15 & 3.30 & 3.43 & 3.73 & 3.99 & 3.24 & 3.36 \\
\hline $\mathrm{Pa}$. & Tioga & 0.00 & 0.00 & 0.00 & 0.00 & 0.00 & 0.00 & 2.27 & 1.63 & 1.46 & 1.30 & 1.69 \\
\hline $\mathrm{Pa}$. & Venango & 3.14 & 4.44 & 4.74 & 4.10 & 0.00 & 0.00 & 6.00 & 6.25 & 9.63 & 7.55 & 5.70 \\
\hline $\mathrm{Pa}$. & Washington & 2.31 & 2.26 & 2.22 & 2.27 & 2.24 & 2.26 & 2.41 & 2.48 & 2.39 & 2.44 & 2.32 \\
\hline $\mathrm{Pa}$. & Westmoreland & 3.30 & 3.50 & 3.75 & 3.49 & 3.82 & 3.71 & 0.00 & 0.00 & 1.59 & 2.83 & 3.46 \\
\hline Tenn. & Anderson & 2.52 & 1.92 & 2.46 & 2.11 & 0.00 & 0.00 & 0.00 & 1.34 & 1.39 & 2.24 & 2.20 \\
\hline Tenn. & Bledsoe & $\mathrm{n} / \mathrm{a}$ & $\mathrm{n} / \mathrm{a}$ & $\mathrm{n} / \mathrm{a}$ & $\mathrm{n} / \mathrm{a}$ & $\mathrm{n} / \mathrm{a}$ & $\mathrm{n} / \mathrm{a}$ & $\mathrm{n} / \mathrm{a}$ & $\mathrm{n} / \mathrm{a}$ & $\mathrm{n} / \mathrm{a}$ & $\mathrm{n} / \mathrm{a}$ & No production \\
\hline Tenn. & Campbell & 1.67 & 1.88 & 0.00 & 0.00 & 0.00 & 1.47 & 1.44 & 1.40 & 1.53 & 2.23 & 1.70 \\
\hline Tenn. & Claiborne & 2.37 & 2.36 & 2.18 & 2.13 & 1.99 & 2.14 & 2.03 & 1.93 & 1.99 & 2.04 & 2.08 \\
\hline Tenn. & Cumberland & 0.00 & 0.00 & 1.28 & 1.49 & 1.63 & 1.15 & 1.11 & 1.21 & 1.43 & 1.17 & 1.32 \\
\hline Tenn. & Fentress & 0.00 & 0.00 & 0.00 & 0.00 & 0.00 & 0.00 & 0.00 & 0.00 & 3.84 & 0.00 & 3.84 \\
\hline Tenn. & Grundy & 0.00 & 0.00 & 0.00 & 0.00 & 0.00 & 0.00 & 0.00 & 0.00 & 0.00 & 3.38 & 3.38 \\
\hline Tenn. & Marion & $\mathrm{n} / \mathrm{a}$ & $\mathrm{n} / \mathrm{a}$ & $\mathrm{n} / \mathrm{a}$ & $\mathrm{n} / \mathrm{a}$ & $\mathrm{n} / \mathrm{a}$ & $\mathrm{n} / \mathrm{a}$ & $\mathrm{n} / \mathrm{a}$ & $\mathrm{n} / \mathrm{a}$ & $\mathrm{n} / \mathrm{a}$ & $\mathrm{n} / \mathrm{a}$ & No production \\
\hline Tenn. & Morgan & 1.89 & 2.31 & 2.46 & 2.44 & 0.00 & 0.00 & 0.00 & 0.00 & 0.00 & 0.00 & 1.93 \\
\hline Tenn. & Rhea & $\mathrm{n} / \mathrm{a}$ & $\mathrm{n} / \mathrm{a}$ & $\mathrm{n} / \mathrm{a}$ & $\mathrm{n} / \mathrm{a}$ & $\mathrm{n} / \mathrm{a}$ & $\mathrm{n} / \mathrm{a}$ & $\mathrm{n} / \mathrm{a}$ & $\mathrm{n} / \mathrm{a}$ & $\mathrm{n} / \mathrm{a}$ & $\mathrm{n} / \mathrm{a}$ & No production \\
\hline Tenn. & Scott & 2.07 & 1.96 & 2.00 & 2.20 & 2.03 & 1.64 & 0.00 & 0.00 & 1.81 & 2.66 & 2.06 \\
\hline Tenn. & Sequatchie & 1.42 & 1.40 & 1.42 & 1.44 & 1.19 & 0.00 & 0.00 & 0.00 & 0.00 & 0.00 & 1.39 \\
\hline Tenn. & Van Buren & $\mathrm{n} / \mathrm{a}$ & $\mathrm{n} / \mathrm{a}$ & $\mathrm{n} / \mathrm{a}$ & $\mathrm{n} / \mathrm{a}$ & $\mathrm{n} / \mathrm{a}$ & $\mathrm{n} / \mathrm{a}$ & $\mathrm{n} / \mathrm{a}$ & $\mathrm{n} / \mathrm{a}$ & $\mathrm{n} / \mathrm{a}$ & $\mathrm{n} / \mathrm{a}$ & No production \\
\hline Va. & Buchanan & 1.31 & 1.24 & 1.18 & 1.24 & 1.14 & 1.19 & 1.24 & 1.30 & 1.33 & 1.38 & 1.25 \\
\hline Va. & Dickenson & 1.69 & 1.70 & 1.45 & 1.75 & 1.60 & 1.57 & 1.51 & 1.71 & 1.73 & 2.10 & 1.68 \\
\hline Va. & Lee & 1.64 & 1.68 & 1.53 & 1.47 & 1.17 & 1.22 & 1.21 & 1.25 & 1.37 & 1.41 & 1.34 \\
\hline
\end{tabular}




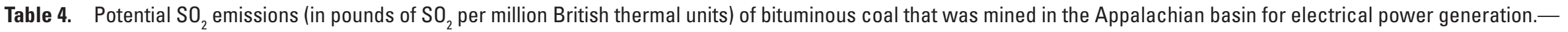
Continued

[Data are shown by county. Data for 1983 through 1995 were calculated from data given in tables 1-3; data for 1996 through 2005 are from the COALdat database. n/a, not available]

\begin{tabular}{|c|c|c|c|c|c|c|c|c|c|c|c|c|c|c|c|}
\hline State & County & 1983 & 1984 & 1985 & 1986 & 1987 & 1988 & 1989 & 1990 & 1991 & 1992 & 1993 & 1994 & 1995 & $\begin{array}{c}\text { Weighted average } \\
\text { (1983-1995) }\end{array}$ \\
\hline Va. & Russell & 1.12 & 1.17 & 1.13 & 1.16 & 1.18 & 1.19 & 1.18 & 1.23 & 1.30 & 1.20 & 1.23 & 1.12 & 1.21 & 1.18 \\
\hline Va. & Scott & 0.00 & 0.00 & 0.00 & 1.55 & 0.00 & 0.00 & 0.00 & 0.00 & 0.00 & 0.00 & 0.00 & 0.00 & 0.00 & 1.55 \\
\hline Va. & Tazewell & 1.20 & 1.16 & 1.39 & 1.27 & 1.18 & 1.12 & 1.81 & 0.85 & 0.00 & 0.00 & 0.00 & 0.00 & 0.00 & 1.27 \\
\hline Va. & Wise & 1.54 & 1.64 & 1.70 & 1.67 & 1.73 & 1.74 & 1.86 & 1.90 & 1.90 & 1.81 & 1.66 & 1.76 & 1.77 & 1.75 \\
\hline W. Va. & Barbour & 4.15 & 3.69 & 3.50 & 3.27 & 3.50 & 3.17 & 2.82 & 2.83 & 2.83 & 2.85 & 2.81 & 2.72 & 1.83 & 3.13 \\
\hline W. Va. & Boone & 1.32 & 1.26 & 1.23 & 1.24 & 1.22 & 1.23 & 1.26 & 1.31 & 1.31 & 1.35 & 1.38 & 1.32 & 1.29 & 1.29 \\
\hline W. Va. & Braxton & 1.22 & 1.17 & 1.09 & 1.20 & 1.17 & 1.23 & 1.42 & 1.30 & 1.36 & 0.00 & 1.65 & 0.00 & 0.00 & 1.23 \\
\hline W. Va. & Brooke & 5.48 & 5.77 & 5.38 & 5.45 & 5.31 & 5.44 & 5.28 & 4.82 & 4.67 & 4.75 & 4.62 & 5.36 & 5.32 & 5.14 \\
\hline W. Va. & Clay & 0.00 & 1.27 & 0.00 & 3.04 & 1.47 & 1.73 & 2.24 & 1.91 & 1.80 & 1.83 & 1.35 & 1.27 & 1.18 & 1.58 \\
\hline W. Va. & Fayette & 1.27 & 1.98 & 1.09 & 1.15 & 1.67 & 2.17 & 2.07 & 1.67 & 1.92 & 2.09 & 1.98 & 1.49 & 1.40 & 1.79 \\
\hline W. Va. & Gilmer & 1.20 & 1.13 & 0.00 & 0.00 & 4.30 & 4.04 & 0.00 & 0.00 & 0.00 & 0.00 & 0.00 & 0.00 & 0.00 & 3.70 \\
\hline W. Va. & Grant & 2.94 & 2.91 & 2.92 & 2.85 & 2.80 & 2.77 & 2.81 & 2.70 & 2.59 & 2.60 & 2.57 & 2.79 & 2.65 & 2.76 \\
\hline W. Va. & Greenbrier & 1.74 & 1.86 & 1.59 & 1.74 & 1.64 & 1.48 & 1.37 & 0.00 & 0.95 & 1.48 & 1.53 & 1.62 & 1.51 & 1.67 \\
\hline W. Va. & Harrison & 4.92 & 4.91 & 5.01 & 4.86 & 4.70 & 4.60 & 4.72 & 4.86 & 4.76 & 4.80 & 4.55 & 4.75 & 5.72 & 4.88 \\
\hline W. Va. & Kanawha & 1.26 & 1.30 & 1.31 & 1.31 & 1.33 & 1.30 & 1.39 & 1.49 & 1.55 & 1.47 & 1.50 & 1.33 & 1.34 & 1.38 \\
\hline W. Va. & Lewis & 4.24 & 4.38 & 4.44 & 4.45 & 4.34 & 5.12 & 4.72 & 2.50 & 0.00 & 0.00 & 3.75 & 4.30 & 4.75 & 4.44 \\
\hline W. Va. & Lincoln & 0.00 & 0.00 & 0.00 & 0.00 & 1.07 & 1.28 & 1.28 & 1.17 & 1.43 & 1.33 & 1.31 & 1.44 & 1.20 & 1.33 \\
\hline W. Va. & Logan & 1.06 & 1.05 & 1.06 & 1.25 & 1.27 & 1.29 & 1.20 & 1.18 & 1.14 & 1.15 & 1.12 & 1.13 & 1.14 & 1.17 \\
\hline W. Va. & McDowell & 1.25 & 1.13 & 1.16 & 1.00 & 1.23 & 1.12 & 1.53 & 1.22 & 1.49 & 0.96 & 1.42 & 0.93 & 1.74 & 1.24 \\
\hline W. Va. & Marion & 3.94 & 3.28 & 3.18 & 2.98 & 2.92 & 2.76 & 2.66 & 2.75 & 3.02 & 2.83 & 3.52 & 2.76 & 3.09 & 3.15 \\
\hline W. Va. & Marshall & 6.27 & 6.29 & 6.37 & 6.31 & 6.45 & 6.47 & 6.64 & 6.81 & 6.74 & 6.48 & 6.00 & 6.41 & 6.12 & 6.44 \\
\hline W. Va. & Mason & 4.24 & 0.00 & 0.00 & 0.00 & 4.89 & 4.89 & 4.95 & 4.11 & 4.70 & 4.39 & 6.24 & 6.29 & 0.00 & 4.89 \\
\hline W. Va. & Mercer & 0.00 & 0.00 & 1.08 & 0.93 & 1.02 & 0.00 & 0.00 & 0.00 & 0.00 & 0.00 & 0.00 & 0.00 & 0.00 & 1.03 \\
\hline W. Va. & Mineral & 2.61 & 3.00 & 3.03 & 2.55 & 2.27 & 2.48 & 2.71 & 2.87 & 2.86 & 2.99 & 3.08 & 2.84 & 2.81 & 2.82 \\
\hline W. Va. & Mingo & 1.30 & 1.21 & 1.10 & 1.17 & 1.19 & 1.16 & 1.21 & 1.23 & 1.24 & 1.21 & 1.24 & 1.24 & 1.18 & 1.21 \\
\hline W. Va. & Monongalia & 3.74 & 4.06 & 3.67 & 3.71 & 3.82 & 3.75 & 3.55 & 3.65 & 3.58 & 3.48 & 3.42 & 3.54 & 3.57 & 3.66 \\
\hline W. Va. & Nicholas & 1.31 & 1.38 & 1.37 & 1.30 & 1.44 & 1.41 & 1.36 & 1.48 & 1.39 & 1.49 & 1.74 & 1.47 & 1.36 & 1.41 \\
\hline W. Va. & Ohio & 2.41 & 0.00 & 5.37 & 5.62 & 4.92 & 5.18 & 4.73 & 5.03 & 4.90 & 4.71 & 5.12 & 6.04 & 4.56 & 5.33 \\
\hline W. Va. & Preston & 2.92 & 3.01 & 2.50 & 2.44 & 2.62 & 2.72 & 2.54 & 2.55 & 2.36 & 2.39 & 2.34 & 2.28 & 2.28 & 2.58 \\
\hline W. Va. & Raleigh & 1.48 & 1.54 & 1.35 & 1.49 & 0.00 & 0.00 & 1.27 & 1.02 & 1.20 & 1.19 & 1.23 & 0.00 & 0.00 & 1.28 \\
\hline
\end{tabular}


Table 4. Potential $\mathrm{SO}_{2}$ emissions (in pounds of $\mathrm{SO}_{2}$ per million British thermal units) of bituminous coal that was mined in the Appalachian basin for electrical power generation.-Continued

[Data are shown by county. Data for 1983 through 1995 were calculated from data given in tables 1-3; data for 1996 through 2005 are from the COALdat database. n/a, not available]

\begin{tabular}{|c|c|c|c|c|c|c|c|c|c|c|c|c|}
\hline State & County & 1996 & 1997 & 1998 & 1999 & 2000 & 2001 & 2002 & 2003 & 2004 & 2005 & $\begin{array}{c}\text { Weighted average } \\
\text { (1996-2005) }\end{array}$ \\
\hline Va. & Russell & 1.13 & 1.19 & 1.20 & 1.17 & 1.13 & 1.21 & 1.19 & 1.14 & 1.21 & 1.38 & 1.19 \\
\hline Va. & Scott & $\mathrm{n} / \mathrm{a}$ & $\mathrm{n} / \mathrm{a}$ & $\mathrm{n} / \mathrm{a}$ & $\mathrm{n} / \mathrm{a}$ & $\mathrm{n} / \mathrm{a}$ & $\mathrm{n} / \mathrm{a}$ & $\mathrm{n} / \mathrm{a}$ & $\mathrm{n} / \mathrm{a}$ & $\mathrm{n} / \mathrm{a}$ & $\mathrm{n} / \mathrm{a}$ & No production \\
\hline Va. & Tazewell & 1.74 & 0.00 & 0.00 & 0.00 & 0.00 & 0.00 & 0.95 & 0.89 & 0.83 & 0.84 & 0.91 \\
\hline Va. & Wise & 1.79 & 1.82 & 1.71 & 1.69 & 1.66 & 1.73 & 1.57 & 1.59 & 1.59 & 1.60 & 1.66 \\
\hline W. Va. & Barbour & 2.85 & 3.67 & 3.86 & 3.15 & 2.65 & 2.01 & 2.73 & 3.81 & 2.55 & 3.92 & 3.18 \\
\hline W. Va. & Boone & 1.30 & 1.27 & 1.26 & 1.27 & 1.27 & 1.28 & 1.27 & 1.34 & 1.35 & 1.31 & 1.29 \\
\hline W. Va. & Braxton & $\mathrm{n} / \mathrm{a}$ & $\mathrm{n} / \mathrm{a}$ & $\mathrm{n} / \mathrm{a}$ & $\mathrm{n} / \mathrm{a}$ & $\mathrm{n} / \mathrm{a}$ & $\mathrm{n} / \mathrm{a}$ & $\mathrm{n} / \mathrm{a}$ & $\mathrm{n} / \mathrm{a}$ & $\mathrm{n} / \mathrm{a}$ & $\mathrm{n} / \mathrm{a}$ & No production \\
\hline W. Va. & Brooke & 5.86 & 6.08 & 6.53 & 6.05 & 6.11 & 6.01 & 6.16 & 0.00 & 0.00 & 0.00 & 6.15 \\
\hline W. Va. & Clay & 1.28 & 1.32 & 1.37 & 1.43 & 1.21 & 1.20 & 1.27 & 1.24 & 1.20 & 1.25 & 1.28 \\
\hline W. Va. & Fayette & 1.63 & 1.64 & 1.72 & 1.75 & 1.89 & 1.81 & 1.20 & 1.07 & 1.94 & 1.33 & 1.66 \\
\hline W. Va. & Gilmer & $\mathrm{n} / \mathrm{a}$ & $\mathrm{n} / \mathrm{a}$ & $\mathrm{n} / \mathrm{a}$ & $\mathrm{n} / \mathrm{a}$ & $\mathrm{n} / \mathrm{a}$ & $\mathrm{n} / \mathrm{a}$ & $\mathrm{n} / \mathrm{a}$ & $\mathrm{n} / \mathrm{a}$ & $\mathrm{n} / \mathrm{a}$ & $\mathrm{n} / \mathrm{a}$ & No production \\
\hline W. Va. & Grant & 2.57 & 2.43 & 2.65 & 2.82 & 2.80 & 2.66 & 2.78 & 2.83 & 3.03 & 2.99 & 2.66 \\
\hline W. Va. & Greenbrier & 0.00 & 0.00 & 0.00 & 1.37 & 1.31 & 1.44 & 1.32 & 0.80 & 0.00 & 0.00 & 1.35 \\
\hline W. Va. & Harrison & 5.39 & 5.51 & 5.43 & 5.31 & 5.23 & 5.29 & 5.30 & 5.25 & 5.69 & 5.75 & 5.41 \\
\hline W. Va. & Kanawha & 1.30 & 1.26 & 1.31 & 1.29 & 1.26 & 1.24 & 1.19 & 1.17 & 1.20 & 1.24 & 1.25 \\
\hline W. Va. & Lewis & $\mathrm{n} / \mathrm{a}$ & $\mathrm{n} / \mathrm{a}$ & $\mathrm{n} / \mathrm{a}$ & $\mathrm{n} / \mathrm{a}$ & $\mathrm{n} / \mathrm{a}$ & $\mathrm{n} / \mathrm{a}$ & $\mathrm{n} / \mathrm{a}$ & $\mathrm{n} / \mathrm{a}$ & $\mathrm{n} / \mathrm{a}$ & $\mathrm{n} / \mathrm{a}$ & No production \\
\hline W. Va. & Lincoln & 0.98 & 1.13 & 1.46 & 2.04 & 1.92 & 1.46 & 1.36 & 1.41 & 1.20 & 1.36 & 1.43 \\
\hline W. Va. & Logan & 1.19 & 1.19 & 1.17 & 1.31 & 1.14 & 1.19 & 1.18 & 1.21 & 1.23 & 1.24 & 1.20 \\
\hline W. Va. & McDowell & 1.16 & 0.00 & 0.00 & 1.03 & 1.17 & 1.23 & 1.20 & 1.04 & 1.18 & 1.22 & 1.16 \\
\hline W. Va. & Marion & 3.82 & 3.16 & 3.27 & 4.31 & 0.00 & 3.25 & 5.05 & 3.90 & 3.67 & 3.71 & 3.56 \\
\hline W. Va. & Marshall & 6.14 & 5.99 & 6.02 & 6.03 & 5.82 & 5.55 & 5.10 & 4.22 & 3.71 & 3.90 & 5.39 \\
\hline W. Va. & Mason & 0.00 & 0.00 & 0.00 & 0.00 & 1.15 & 0.00 & 0.00 & 0.00 & 1.13 & 0.00 & 1.14 \\
\hline W. Va. & Mercer & 0.00 & 0.00 & 0.00 & 0.00 & 0.00 & 0.00 & 1.72 & 0.00 & 0.00 & 0.00 & 1.72 \\
\hline W. Va. & Mineral & 2.76 & 0.00 & 0.00 & 0.00 & 2.83 & 0.00 & 0.00 & 0.00 & 0.00 & 3.39 & 2.84 \\
\hline W. Va. & Mingo & 1.24 & 1.25 & 1.23 & 1.19 & 1.17 & 1.19 & 1.22 & 1.34 & 1.32 & 1.17 & 1.23 \\
\hline W. Va. & Monongalia & 3.38 & 3.29 & 3.51 & 3.50 & 3.52 & 3.52 & 3.40 & 3.46 & 3.73 & 3.48 & 3.47 \\
\hline W. Va. & Nicholas & 1.44 & 1.14 & 1.32 & 1.65 & 1.37 & 1.30 & 1.65 & 1.57 & 1.54 & 1.64 & 1.50 \\
\hline W. Va. & Ohio & 0.00 & 0.00 & 0.00 & 0.00 & 6.93 & 0.00 & 0.00 & 0.00 & 0.00 & 0.00 & 6.93 \\
\hline W. Va. & Preston & 2.19 & 2.28 & 2.30 & 2.10 & 2.24 & 2.20 & 2.58 & 2.50 & 2.54 & 2.42 & 2.35 \\
\hline W. Va. & Raleigh & 1.28 & 1.83 & 1.73 & 1.77 & 2.02 & 1.84 & 1.37 & 1.53 & 2.21 & 2.09 & 1.84 \\
\hline
\end{tabular}




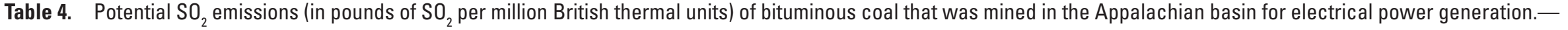
Continued

[Data are shown by county. Data for 1983 through 1995 were calculated from data given in tables 1-3; data for 1996 through 2005 are from the COALdat database. n/a, not available]

\begin{tabular}{|c|c|c|c|c|c|c|c|c|c|c|c|c|c|c|c|}
\hline State & County & 1983 & 1984 & 1985 & 1986 & 1987 & 1988 & 1989 & 1990 & 1991 & 1992 & 1993 & 1994 & 1995 & $\begin{array}{c}\text { Weighted average } \\
\text { (1983-1995) }\end{array}$ \\
\hline W. Va. & Randolph & 1.68 & 1.71 & 1.63 & 1.73 & 1.80 & 1.95 & 1.97 & 2.39 & 2.29 & 1.70 & 2.58 & 1.34 & 1.84 & 1.85 \\
\hline W. Va. & Taylor & 0.00 & 0.00 & 2.28 & 2.32 & 0.00 & 2.53 & 2.66 & 2.77 & 0.00 & 2.60 & 2.86 & 0.00 & 0.00 & 2.51 \\
\hline W. Va. & Tucker & 3.42 & 3.11 & 3.32 & 2.75 & 0.00 & 0.00 & 0.00 & 0.00 & 0.00 & 0.00 & 0.00 & 0.00 & 0.00 & 3.05 \\
\hline W. Va. & Upshur & 1.82 & 1.80 & 1.83 & 1.80 & 2.34 & 3.93 & 3.53 & 3.20 & 4.03 & 4.40 & 3.41 & 3.75 & 3.96 & 2.51 \\
\hline W. Va. & Wayne & 1.57 & 0.00 & 3.99 & 1.92 & 3.24 & 1.85 & 1.41 & 1.14 & 1.11 & 1.40 & 1.50 & 1.45 & 1.39 & 1.44 \\
\hline W. Va. & Webster & 1.01 & 1.06 & 1.16 & 1.18 & 1.29 & 1.31 & 1.27 & 1.25 & 1.25 & 1.31 & 1.50 & 1.30 & 1.32 & 1.30 \\
\hline W. Va. & Wyoming & 1.43 & 1.60 & 1.37 & 1.40 & 1.13 & 1.16 & 1.06 & 0.00 & 0.00 & 0.00 & 0.94 & 1.04 & 1.15 & 1.35 \\
\hline
\end{tabular}


Table 4. Potential $\mathrm{SO}_{2}$ emissions (in pounds of $\mathrm{SO}_{2}$ per million British thermal units) of bituminous coal that was mined in the Appalachian basin for electrical power generation.-Continued

[Data are shown by county. Data for 1983 through 1995 were calculated from data given in tables 1-3; data for 1996 through 2005 are from the COALdat database. n/a, not available]

\begin{tabular}{|c|c|c|c|c|c|c|c|c|c|c|c|c|}
\hline State & County & 1996 & 1997 & 1998 & 1999 & 2000 & 2001 & 2002 & 2003 & 2004 & 2005 & $\begin{array}{l}\text { Weighted average } \\
\text { (1996-2005) }\end{array}$ \\
\hline W. Va. & Randolph & 0.00 & 0.00 & 0.00 & 0.00 & 0.00 & 0.00 & 0.00 & 1.13 & 0.00 & 0.00 & 1.13 \\
\hline W. Va. & Taylor & $\mathrm{n} / \mathrm{a}$ & $\mathrm{n} / \mathrm{a}$ & $\mathrm{n} / \mathrm{a}$ & $\mathrm{n} / \mathrm{a}$ & $\mathrm{n} / \mathrm{a}$ & $\mathrm{n} / \mathrm{a}$ & $\mathrm{n} / \mathrm{a}$ & $\mathrm{n} / \mathrm{a}$ & $\mathrm{n} / \mathrm{a}$ & $\mathrm{n} / \mathrm{a}$ & No production \\
\hline W. Va. & Tucker & $\mathrm{n} / \mathrm{a}$ & $\mathrm{n} / \mathrm{a}$ & $\mathrm{n} / \mathrm{a}$ & $\mathrm{n} / \mathrm{a}$ & $\mathrm{n} / \mathrm{a}$ & $\mathrm{n} / \mathrm{a}$ & $\mathrm{n} / \mathrm{a}$ & $\mathrm{n} / \mathrm{a}$ & $\mathrm{n} / \mathrm{a}$ & $\mathrm{n} / \mathrm{a}$ & No production \\
\hline W. Va. & Upshur & 3.86 & 3.23 & 2.53 & 2.30 & 2.37 & 2.40 & 3.43 & 3.83 & 3.44 & 2.61 & 2.84 \\
\hline W. Va. & Wayne & 1.33 & 1.31 & 1.34 & 1.35 & 1.30 & 1.32 & 1.34 & 1.25 & 1.31 & 1.40 & 1.33 \\
\hline W. Va. & Webster & 1.31 & 1.37 & 1.39 & 1.47 & 1.54 & 1.52 & 1.72 & 1.75 & 1.81 & 1.76 & 1.55 \\
\hline W. Va. & Wyoming & 1.78 & 1.02 & 1.14 & 1.04 & 1.16 & 1.16 & 1.52 & 1.32 & 1.25 & 1.44 & 1.30 \\
\hline
\end{tabular}


Table 5. Summary statistics comparing weighted average sulfur content (in weight percent) and potential $\mathrm{SO}_{2}$ emissions (in pounds of $\mathrm{SO}_{2}$ per million British thermal units) for 1983 through 1995 and 1996 through 2005.

[Data are shown by county. Data shown in columns 3 and 4 are from table 3; data shown in columns 8 and 9 are from table $4 . \mathrm{SO}_{2}$, sulfur dioxide; lb $\mathrm{SO}_{2} / \mathrm{MMBtu}$, pounds of sulfur dioxide per million British thermal units]

\begin{tabular}{|c|c|c|c|c|c|c|c|c|c|c|c|}
\hline State & County & $\begin{array}{l}\text { Weighted } \\
\text { average } \\
\text { sulfur for } \\
1983-1995\end{array}$ & $\begin{array}{c}\text { Weighted } \\
\text { average sulfur } \\
\text { for 1996-2005 }\end{array}$ & $\begin{array}{c}\text { Change in } \\
\text { weighted } \\
\text { average sulfur } \\
\text { (weight percent) }\end{array}$ & $\begin{array}{l}\text { Decrease or } \\
\text { increase }\end{array}$ & $\begin{array}{l}\text { Decrease } \\
\text { or increase } \\
\text { greater } \\
\text { than } 1\end{array}$ & $\begin{array}{c}\text { Weighted average } \\
\text { of potential } \mathrm{SO}_{2} \\
\text { emissions for } \\
1983-1995\end{array}$ & $\begin{array}{l}\text { Weighted aver- } \\
\text { age of potential } \\
\mathrm{SO}_{2} \text { emissions for } \\
1996-2005\end{array}$ & $\begin{array}{l}\text { Change in } \\
\text { weighted aver- } \\
\text { age of potential } \\
\mathrm{SO}_{2} \text { emissions } \\
\left.\text { (lb } \mathrm{SO}_{2} / \mathrm{MMBtu}\right)\end{array}$ & $\begin{array}{l}\text { Decrease or } \\
\text { increase }\end{array}$ & $\begin{array}{c}\text { Decrease } \\
\text { or increase } \\
\text { greater } \\
\text { than } 1\end{array}$ \\
\hline Ala. & Bibb & 0.73 & 1.50 & 0.77 & Increase & & 1.42 & 2.49 & 1.07 & Increase & 1.07 \\
\hline Ala. & Blount & 2.21 & No production & No production & No production & & 3.61 & No production & No production & No production & \\
\hline Ala. & Cullman & 0.74 & No production & No production & No production & & 1.12 & No production & No production & No production & \\
\hline Ala. & De Kalb & 1.18 & No production & No production & No production & & 1.87 & No production & No production & No production & \\
\hline Ala. & Fayette & 2.08 & 1.65 & -0.43 & Decrease & & 3.45 & 2.73 & -0.72 & Decrease & \\
\hline Ala. & Jackson & 1.27 & 0.77 & -0.50 & Decrease & & 2.00 & 1.29 & -0.71 & Decrease & \\
\hline Ala. & Jefferson & 1.23 & 0.84 & -0.39 & Decrease & & 1.98 & 1.36 & -0.62 & Decrease & \\
\hline Ala. & Lamar & 1.37 & No production & No production & No production & & 2.27 & No production & No production & No production & \\
\hline Ala. & Marion & 1.30 & No production & No production & No production & & 2.21 & No production & No production & No production & \\
\hline Ala. & Marshall & 1.94 & No production & No production & No production & & 3.25 & No production & No production & No production & \\
\hline Ala. & St. Clair & 1.31 & No production & No production & No production & & 2.11 & No production & No production & No production & \\
\hline Ala. & Shelby & 0.84 & 0.70 & -0.14 & Decrease & & 1.33 & 1.11 & -0.22 & Decrease & \\
\hline Ala. & Tuscaloosa & 1.19 & 1.35 & 0.16 & Increase & & 1.92 & 2.20 & 0.28 & Increase & \\
\hline Ala. & Walker & 1.14 & 1.53 & 0.39 & Increase & & 1.90 & 2.57 & 0.67 & Increase & \\
\hline Ala. & Winston & 1.31 & No production & No production & No production & & 2.17 & No production & No production & No production & \\
\hline Ga. & Dade & 1.60 & No production & No production & No production & & 2.43 & No production & No production & No production & \\
\hline Ky. & Bell & 1.28 & 1.29 & 0.01 & Increase & & 2.05 & 2.04 & -0.01 & Decrease & \\
\hline Ky. & Boyd & 1.15 & 0.90 & -0.25 & Decrease & & 1.96 & 1.50 & -0.46 & Decrease & \\
\hline Ку. & Breathitt & 0.99 & 1.35 & 0.36 & Increase & & 1.68 & 2.22 & 0.54 & Increase & \\
\hline Ку. & Carter & 1.14 & 0.86 & -0.28 & Decrease & & 2.01 & 1.37 & -0.64 & Decrease & \\
\hline Ky. & Clay & 1.54 & 1.26 & -0.28 & Decrease & & 2.50 & 2.05 & -0.45 & Decrease & \\
\hline Ky. & Clinton & 0.84 & No production & No production & No production & & 1.26 & No production & No production & No production & \\
\hline Ky. & Elliott & 1.57 & No production & No production & No production & & 2.73 & No production & No production & No production & \\
\hline Ky. & Estill & 0.88 & 1.03 & 0.15 & Increase & & 1.41 & 1.73 & 0.32 & Increase & \\
\hline Ky. & Floyd & 1.04 & 1.05 & 0.01 & Increase & & 1.69 & 1.71 & 0.02 & Increase & \\
\hline
\end{tabular}




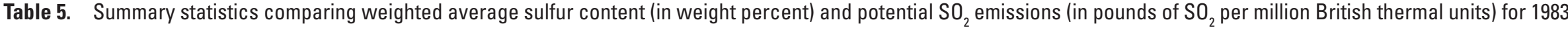
through 1995 and 1996 through 2005.-Continued

[Data are shown by county. Data shown in columns 3 and 4 are from table 3; data shown in columns 8 and 9 are from table $4 . \mathrm{SO}_{2}$, sulfur dioxide; lb $\mathrm{SO}_{2} / \mathrm{MMBtu}$, pounds of sulfur dioxide per million British thermal units]

\begin{tabular}{|c|c|c|c|c|c|c|c|c|c|c|c|}
\hline State & County & $\begin{array}{l}\text { Weighted } \\
\text { average } \\
\text { sulfur for } \\
\text { 1983-1995 }\end{array}$ & $\begin{array}{l}\text { Weighted } \\
\text { average sulfur } \\
\text { for 1996-2005 }\end{array}$ & $\begin{array}{c}\text { Change in } \\
\text { weighted } \\
\text { average sulfur } \\
\text { (weight percent) }\end{array}$ & $\begin{array}{l}\text { Decrease or } \\
\text { increase }\end{array}$ & $\begin{array}{l}\text { Decrease } \\
\text { or increase } \\
\text { greater } \\
\text { than } 1\end{array}$ & $\begin{array}{c}\text { Weighted average } \\
\text { of potential } \mathrm{SO}_{2} \\
\text { emissions for } \\
1983-1995\end{array}$ & $\begin{array}{l}\text { Weighted aver- } \\
\text { age of potential } \\
\mathrm{SO}_{2} \text { emissions for } \\
\text { 1996-2005 }\end{array}$ & $\begin{array}{l}\text { Change in } \\
\text { weighted aver- } \\
\text { age of potential } \\
\mathrm{SO}_{2} \text { emissions } \\
\text { (lb SO} \mathrm{O}_{2} / \mathrm{MMBtu} \text { ) }\end{array}$ & $\begin{array}{l}\text { Decrease or } \\
\text { increase }\end{array}$ & $\begin{array}{c}\text { Decrease } \\
\text { or increase } \\
\text { greater } \\
\text { than } 1\end{array}$ \\
\hline Ky. & Greenup & 2.11 & 0.85 & -1.26 & Decrease & -1.26 & 3.67 & 1.38 & -2.29 & Decrease & -2.29 \\
\hline Ky. & Harlan & 1.10 & 1.05 & -0.05 & Decrease & & 1.75 & 1.65 & -0.10 & Decrease & \\
\hline Ky. & Jackson & 1.29 & 0.83 & -0.46 & Decrease & & 2.13 & 1.33 & -0.80 & Decrease & \\
\hline Ky. & Johnson & 1.21 & 1.19 & -0.02 & Decrease & & 2.06 & 2.01 & -0.05 & Decrease & \\
\hline Ky. & Knott & 1.07 & 1.12 & 0.05 & Increase & & 1.72 & 1.79 & 0.07 & Increase & \\
\hline Ky. & Knox & 1.36 & 1.68 & 0.32 & Increase & & 2.18 & 2.80 & 0.62 & Increase & \\
\hline Ky. & Laurel & 1.48 & 0.96 & -0.52 & Decrease & & 2.43 & 1.58 & -0.85 & Decrease & \\
\hline Ky. & Lawrence & 1.19 & 1.25 & 0.06 & Increase & & 2.07 & 2.13 & 0.06 & Increase & \\
\hline Ky. & Lee & 1.84 & 0.91 & -0.93 & Decrease & & 3.10 & 1.53 & -1.57 & Decrease & -1.57 \\
\hline Ky. & Leslie & 1.30 & 1.07 & -0.23 & Decrease & & 2.10 & 1.70 & -0.40 & Decrease & \\
\hline Ky. & Letcher & 0.92 & 1.21 & 0.29 & Increase & & 1.43 & 1.88 & 0.45 & Increase & \\
\hline Ky. & McCreary & 1.49 & 0.93 & -0.56 & Decrease & & 2.39 & 1.42 & -0.97 & Decrease & \\
\hline Ky. & Magoffin & 1.08 & 0.95 & -0.13 & Decrease & & 1.80 & 1.59 & -0.21 & Decrease & \\
\hline Ky. & Martin & 0.90 & 0.90 & 0.00 & No change & & 1.46 & 1.48 & 0.02 & Increase & \\
\hline Ky. & Morgan & 1.14 & 1.00 & -0.14 & Decrease & & 1.97 & 1.78 & -0.19 & Decrease & \\
\hline Ky. & Owsley & 1.71 & 1.47 & -0.24 & Decrease & & 2.91 & 2.42 & -0.49 & Decrease & \\
\hline Ky. & Perry & 1.08 & 1.01 & -0.07 & Decrease & & 1.77 & 1.64 & -0.13 & Decrease & \\
\hline Ky. & Pike & 0.93 & 1.00 & 0.07 & Increase & & 1.47 & 1.59 & 0.12 & Increase & \\
\hline Ky. & Pulaski & 1.67 & 1.28 & -0.39 & Decrease & & 2.76 & 2.13 & -0.63 & Decrease & \\
\hline Ky. & Rockcastle & 1.21 & 0.78 & -0.43 & Decrease & & 2.09 & 1.32 & -0.77 & Decrease & \\
\hline Ky. & Wayne & 0.59 & No production & No production & No production & & 1.01 & No production & No production & No production & \\
\hline Ky. & Whitley & 1.18 & 1.10 & -0.08 & Decrease & & 1.86 & 1.74 & -0.12 & Decrease & \\
\hline Ky. & Wolfe & 2.21 & 0.94 & -1.27 & Decrease & -1.27 & 3.72 & 1.65 & -2.07 & Decrease & -2.07 \\
\hline Md. & Allegany & 1.66 & 1.57 & -0.09 & Decrease & & 2.79 & 2.69 & -0.10 & Decrease & \\
\hline Md. & Garrett & 1.61 & 1.69 & 0.08 & Increase & & 2.54 & 2.74 & 0.20 & Increase & \\
\hline
\end{tabular}




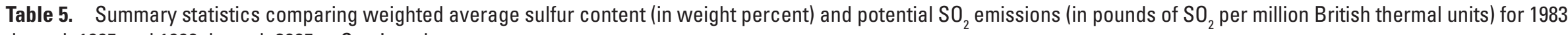
through 1995 and 1996 through 2005.-Continued

[Data are shown by county. Data shown in columns 3 and 4 are from table 3; data shown in columns 8 and 9 are from table 4 . $\mathrm{SO}_{2}$, sulfur dioxide; lb $\mathrm{SO}_{2} / \mathrm{MMBtu}$, pounds of sulfur dioxide per million British thermal units]

\begin{tabular}{|c|c|c|c|c|c|c|c|c|c|c|c|}
\hline State & County & $\begin{array}{l}\text { Weighted } \\
\text { average } \\
\text { sulfur for } \\
\text { 1983-1995 }\end{array}$ & $\begin{array}{c}\text { Weighted } \\
\text { average sulfur } \\
\text { for 1996-2005 }\end{array}$ & $\begin{array}{c}\text { Change in } \\
\text { weighted } \\
\text { average sulfur } \\
\text { (weight percent) }\end{array}$ & $\begin{array}{l}\text { Decrease or } \\
\text { increase }\end{array}$ & $\begin{array}{l}\text { Decrease } \\
\text { or increase } \\
\text { greater } \\
\text { than } 1\end{array}$ & $\begin{array}{c}\text { Weighted average } \\
\text { of potential } \mathrm{SO}_{2} \\
\text { emissions for } \\
1983-1995\end{array}$ & $\begin{array}{l}\text { Weighted aver- } \\
\text { age of potential } \\
\mathrm{SO}_{2} \text { emissions for } \\
1996-2005\end{array}$ & $\begin{array}{c}\text { Change in } \\
\text { weighted aver- } \\
\text { age of potential } \\
\mathrm{SO}_{2} \text { emissions } \\
\left.\text { (lb SO } \mathrm{O}_{2} / \mathrm{MMBtu}\right)\end{array}$ & $\begin{array}{c}\text { Decrease or } \\
\text { increase }\end{array}$ & $\begin{array}{l}\text { Decrease } \\
\text { or increase } \\
\text { greater } \\
\text { than } 1\end{array}$ \\
\hline Ohio & Athens & 2.33 & 1.70 & -0.63 & Decrease & & 4.03 & 3.06 & -0.97 & Decrease & \\
\hline Ohio & Belmont & 3.26 & 3.90 & 0.64 & Increase & & 5.41 & 6.30 & 0.89 & Increase & \\
\hline Ohio & Carroll & 2.66 & 1.74 & -0.92 & Decrease & & 4.44 & 2.82 & -1.62 & Decrease & -1.62 \\
\hline Ohio & Columbiana & 2.43 & 2.39 & -0.04 & Decrease & & 3.99 & 3.89 & -0.10 & Decrease & \\
\hline Ohio & Coshocton & 3.13 & 2.44 & -0.69 & Decrease & & 5.22 & 4.13 & -1.09 & Decrease & -1.09 \\
\hline Ohio & Gallia & 3.20 & 3.60 & 0.40 & Increase & & 5.58 & 5.89 & 0.31 & Increase & \\
\hline Ohio & Guernsey & 3.33 & 4.53 & 1.20 & Increase & 1.20 & 5.84 & 7.89 & 2.05 & Increase & 2.05 \\
\hline Ohio & Harrison & 2.86 & 2.82 & -0.04 & Decrease & & 4.63 & 4.62 & -0.01 & Decrease & \\
\hline Ohio & Hocking & 3.39 & No production & No production & No production & & 6.07 & No production & No production & No production & \\
\hline Ohio & Holmes & 3.56 & 3.62 & 0.06 & Increase & & 6.14 & 6.28 & 0.14 & Increase & \\
\hline Ohio & Jackson & 3.22 & 3.40 & 0.18 & Increase & & 5.76 & 6.10 & 0.34 & Increase & \\
\hline Ohio & Jefferson & 2.92 & 2.48 & -0.44 & Decrease & & 4.90 & 4.17 & -0.73 & Decrease & \\
\hline Ohio & Lawrence & 2.71 & 2.84 & 0.13 & Increase & & 4.73 & 4.91 & 0.18 & Increase & \\
\hline Ohio & Mahoning & 3.15 & No production & No production & No production & & 5.22 & No production & No production & No production & \\
\hline Ohio & Meigs & 3.34 & 3.48 & 0.14 & Increase & & 5.93 & 6.16 & 0.23 & Increase & \\
\hline Ohio & Monroe & 4.00 & 4.30 & 0.30 & Increase & & 6.60 & 6.96 & 0.36 & Increase & \\
\hline Ohio & Morgan & 4.16 & No production & No production & No production & & 7.29 & No production & No production & No production & \\
\hline Ohio & Muskingum & 4.25 & 3.61 & -0.64 & Decrease & & 7.43 & 6.30 & -1.13 & Decrease & -1.13 \\
\hline Ohio & Noble & 4.58 & 3.64 & -0.94 & Decrease & & 8.00 & 6.29 & -1.71 & Decrease & -1.71 \\
\hline Ohio & Perry & 2.38 & 3.35 & 0.97 & Increase & & 4.21 & 5.80 & 1.59 & Increase & 1.59 \\
\hline Ohio & Pike & 2.60 & No production & No production & No production & & 4.35 & No production & No production & No production & \\
\hline Ohio & Scioto & 1.08 & 1.93 & 0.85 & Increase & & 1.86 & 3.08 & 1.22 & Increase & 1.22 \\
\hline Ohio & Stark & 3.49 & 3.24 & -0.25 & Decrease & & 5.95 & 5.48 & -0.47 & Decrease & \\
\hline Ohio & Tuscarawas & 3.24 & 3.45 & 0.21 & Increase & & 5.45 & 6.10 & 0.65 & Increase & \\
\hline Ohio & Vinton & 3.18 & 2.99 & -0.19 & Decrease & & 5.65 & 5.26 & -0.39 & Decrease & \\
\hline
\end{tabular}


Table 5. Summary statistics comparing weighted average sulfur content (in weight percent) and potential $\mathrm{SO}_{2}$ emissions (in pounds of $\mathrm{SO}_{2}$ per million $\mathrm{British}$ thermal units) for 1983 through 1995 and 1996 through 2005.-Continued

[Data are shown by county. Data shown in columns 3 and 4 are from table 3; data shown in columns 8 and 9 are from table $4 . \mathrm{SO}_{2}$, sulfur dioxide; lb $\mathrm{SO}_{2} / \mathrm{MMBtu}$, pounds of sulfur dioxide per million British thermal units]

\begin{tabular}{|c|c|c|c|c|c|c|c|c|c|c|c|}
\hline State & County & $\begin{array}{l}\text { Weighted } \\
\text { average } \\
\text { sulfur for } \\
1983-1995\end{array}$ & $\begin{array}{l}\text { Weighted } \\
\text { average sulfur } \\
\text { for 1996-2005 }\end{array}$ & $\begin{array}{c}\text { Change in } \\
\text { weighted } \\
\text { average sulfur } \\
\text { (weight percent) }\end{array}$ & $\begin{array}{l}\text { Decrease or } \\
\text { increase }\end{array}$ & $\begin{array}{l}\text { Decrease } \\
\text { or increase } \\
\text { greater } \\
\text { than } 1\end{array}$ & $\begin{array}{c}\text { Weighted average } \\
\text { of potential } \mathrm{SO}_{2} \\
\text { emissions for } \\
1983-1995\end{array}$ & $\begin{array}{l}\text { Weighted aver- } \\
\text { age of potential } \\
\mathrm{SO}_{2} \text { emissions for } \\
1996-2005\end{array}$ & $\begin{array}{l}\text { Change in } \\
\text { weighted aver- } \\
\text { age of potential } \\
\mathrm{SO}_{2} \text { emissions } \\
\left.\text { (Ib } \mathrm{SO}_{2} / \mathrm{MMBtu}\right)\end{array}$ & $\begin{array}{c}\text { Decrease or } \\
\text { increase }\end{array}$ & $\begin{array}{c}\text { Decrease } \\
\text { or increase } \\
\text { greater } \\
\text { than } 1\end{array}$ \\
\hline Ohio & Washington & 0.00 & 2.63 & 2.63 & Increase & 2.63 & 0.00 & 4.05 & 4.05 & Increase & 4.05 \\
\hline $\mathrm{Pa}$. & Allegheny & 1.40 & 1.50 & 0.10 & Increase & & 2.25 & 2.56 & 0.31 & Increase & \\
\hline $\mathrm{Pa}$. & Armstrong & 1.70 & 2.22 & 0.52 & Increase & & 2.74 & 3.60 & 0.86 & Increase & \\
\hline $\mathrm{Pa}$. & Beaver & 2.40 & 2.19 & -0.21 & Decrease & & 3.97 & 3.64 & -0.33 & Decrease & \\
\hline $\mathrm{Pa}$. & Bedford & 1.90 & 0.95 & -0.95 & Decrease & & 3.01 & 1.76 & -1.25 & Decrease & -1.25 \\
\hline $\mathrm{Pa}$. & Blair & 1.81 & 2.54 & 0.73 & Increase & & 2.76 & 4.58 & 1.82 & Increase & 1.82 \\
\hline $\mathrm{Pa}$. & Bradford & 0.82 & No production & No production & No production & & 1.57 & No production & No production & No production & \\
\hline $\mathrm{Pa}$. & Butler & 2.08 & 1.88 & -0.20 & Decrease & & 3.36 & 3.15 & -0.21 & Decrease & \\
\hline $\mathrm{Pa}$. & Cambria & 1.64 & 2.21 & 0.57 & Increase & & 2.64 & 5.71 & 3.07 & Increase & 3.07 \\
\hline $\mathrm{Pa}$. & Cameron & 0.58 & No production & No production & No production & & 1.00 & No production & No production & No production & \\
\hline $\mathrm{Pa}$. & Centre & 1.77 & 1.52 & -0.25 & Decrease & & 2.89 & 2.42 & -0.47 & Decrease & \\
\hline $\mathrm{Pa}$. & Clarion & 2.21 & 1.67 & -0.54 & Decrease & & 3.48 & 2.71 & -0.77 & Decrease & \\
\hline $\mathrm{Pa}$. & Clearfield & 1.86 & 1.80 & -0.06 & Decrease & & 2.98 & 2.97 & -0.01 & Decrease & \\
\hline $\mathrm{Pa}$. & Clinton & 1.01 & No production & No production & No production & & 1.73 & No production & No production & No production & \\
\hline $\mathrm{Pa}$. & Elk & 1.75 & 1.74 & -0.01 & Decrease & & 2.79 & 3.18 & 0.39 & Increase & \\
\hline $\mathrm{Pa}$. & Fayette & 1.85 & 1.82 & -0.03 & Decrease & & 2.99 & 3.00 & 0.01 & Increase & \\
\hline $\mathrm{Pa}$. & Fulton & 2.01 & 2.12 & 0.11 & Increase & & 3.19 & 3.39 & 0.20 & Increase & \\
\hline $\mathrm{Pa}$. & Greene & 1.77 & 1.92 & 0.15 & Increase & & 2.73 & 2.96 & 0.23 & Increase & \\
\hline $\mathrm{Pa}$. & Huntingdon & 1.91 & No production & No production & No production & & 3.43 & No production & No production & No production & \\
\hline $\mathrm{Pa}$. & Indiana & 1.92 & 2.11 & 0.19 & Increase & & 3.17 & 3.62 & 0.45 & Increase & \\
\hline $\mathrm{Pa}$. & Jefferson & 1.71 & 1.74 & 0.03 & Increase & & 2.70 & 2.81 & 0.11 & Increase & \\
\hline $\mathrm{Pa}$. & Lawrence & 2.26 & 2.43 & 0.17 & Increase & & 3.78 & 4.69 & 0.91 & Increase & \\
\hline $\mathrm{Pa}$. & Lycoming & 0.90 & 0.78 & -0.12 & Decrease & & 1.59 & 1.44 & -0.15 & Decrease & \\
\hline $\mathrm{Pa}$. & Mercer & 1.72 & 5.93 & 4.21 & Increase & 4.21 & 2.72 & 10.90 & 8.18 & Increase & 8.18 \\
\hline $\mathrm{Pa}$. & Somerset & 1.73 & 2.06 & 0.33 & Increase & & 2.79 & 3.36 & 0.57 & Increase & \\
\hline
\end{tabular}




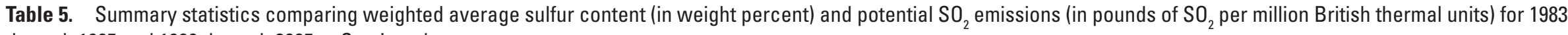
through 1995 and 1996 through 2005.-Continued

[Data are shown by county. Data shown in columns 3 and 4 are from table 3; data shown in columns 8 and 9 are from table $4 . \mathrm{SO}_{2}$, sulfur dioxide; $1 \mathrm{~b} \mathrm{SO} / \mathrm{MMBtu}_{2}$ pounds of sulfur dioxide per million British thermal units]

\begin{tabular}{|c|c|c|c|c|c|c|c|c|c|c|c|}
\hline State & County & $\begin{array}{l}\text { Weighted } \\
\text { average } \\
\text { sulfur for } \\
\text { 1983-1995 }\end{array}$ & $\begin{array}{c}\text { Weighted } \\
\text { average sulfur } \\
\text { for 1996-2005 }\end{array}$ & $\begin{array}{c}\text { Change in } \\
\text { weighted } \\
\text { average sulfur } \\
\text { (weight percent) }\end{array}$ & $\begin{array}{l}\text { Decrease or } \\
\text { increase }\end{array}$ & $\begin{array}{l}\text { Decrease } \\
\text { or increase } \\
\text { greater } \\
\text { than } 1\end{array}$ & $\begin{array}{c}\text { Weighted average } \\
\text { of potential } \mathrm{SO}_{2} \\
\text { emissions for } \\
1983-1995\end{array}$ & $\begin{array}{l}\text { Weighted aver- } \\
\text { age of potential } \\
\mathrm{SO}_{2} \text { emissions for } \\
1996-2005\end{array}$ & $\begin{array}{l}\text { Change in } \\
\text { weighted aver- } \\
\text { age of potential } \\
\mathrm{SO}_{2} \text { emissions } \\
\left.\text { (lb SO} \mathrm{I}_{2} / \mathrm{MMBtu}\right)\end{array}$ & $\begin{array}{l}\text { Decrease or } \\
\text { increase }\end{array}$ & $\begin{array}{l}\text { Decrease } \\
\text { or increase } \\
\text { greater } \\
\text { than } 1\end{array}$ \\
\hline Pa. & Tioga & 1.10 & 0.63 & -0.47 & Decrease & & 1.96 & 1.69 & -0.27 & Decrease & \\
\hline Pa. & Venango & 2.64 & 2.94 & 0.30 & Increase & & 4.11 & 5.70 & 1.59 & Increase & 1.59 \\
\hline Pa. & Washington & 2.11 & 1.52 & -0.59 & Decrease & & 3.33 & 2.32 & -1.01 & Decrease & -1.01 \\
\hline Pa. & Westmoreland & 1.92 & 2.11 & 0.19 & Increase & & 3.13 & 3.46 & 0.33 & Increase & \\
\hline Tenn. & Anderson & 1.85 & 1.35 & -0.50 & Decrease & & 2.97 & 2.20 & -0.77 & Decrease & \\
\hline Tenn. & Bledsoe & 1.18 & No production & No production & No production & & 1.88 & No production & No production & No production & \\
\hline Tenn. & Campbell & 1.48 & 1.07 & -0.41 & Decrease & & 2.37 & 1.70 & -0.67 & Decrease & \\
\hline Tenn. & Claiborne & 1.01 & 1.34 & 0.33 & Increase & & 1.58 & 2.08 & 0.50 & Increase & \\
\hline Tenn. & Cumberland & 1.07 & 0.83 & -0.24 & Decrease & & 1.77 & 1.32 & -0.45 & Decrease & \\
\hline Tenn. & Fentress & 2.14 & 2.21 & 0.07 & Increase & & 3.60 & 3.84 & 0.24 & Increase & \\
\hline Tenn. & Grundy & 0.88 & 2.17 & 1.29 & Increase & 1.29 & 1.43 & 3.38 & 1.95 & Increase & 1.95 \\
\hline Tenn. & Marion & 0.81 & No production & No production & No production & & 1.30 & No production & No production & No production & \\
\hline Tenn. & Morgan & 1.48 & 1.23 & -0.25 & Decrease & & 2.33 & 1.93 & -0.40 & Decrease & \\
\hline Tenn. & Rhea & 1.60 & No production & No production & No production & & 2.61 & No production & No production & No production & \\
\hline Tenn. & Scott & 1.59 & 1.31 & -0.28 & Decrease & & 2.46 & 2.06 & -0.40 & Decrease & \\
\hline Tenn. & Sequatchie & 0.92 & 0.86 & -0.06 & Decrease & & 1.50 & 1.39 & -0.11 & Decrease & \\
\hline Tenn. & Van Buren & 1.80 & No production & No production & No production & & 3.15 & No production & No production & No production & \\
\hline Va. & Buchanan & 1.02 & 0.83 & -0.19 & Decrease & & 1.55 & 1.25 & -0.30 & Decrease & \\
\hline Va. & Dickenson & 1.10 & 1.08 & -0.02 & Decrease & & 1.70 & 1.68 & -0.02 & Decrease & \\
\hline Va. & Lee & 1.04 & 0.84 & -0.20 & Decrease & & 1.64 & 1.34 & -0.30 & Decrease & \\
\hline Va. & Russell & 0.75 & 0.74 & -0.01 & Decrease & & 1.18 & 1.19 & 0.01 & Increase & \\
\hline Va. & Scott & 1.00 & No production & No production & No production & & 1.55 & No production & No production & No production & \\
\hline Va. & Tazewell & 0.85 & 0.58 & -0.27 & Decrease & & 1.27 & 0.91 & -0.36 & Decrease & \\
\hline Va. & Wise & 1.12 & 1.06 & -0.06 & Decrease & & 1.75 & 1.66 & -0.09 & Decrease & \\
\hline W. Va. & Barbour & 2.00 & 2.04 & 0.04 & Increase & & 3.13 & 3.18 & 0.05 & Increase & \\
\hline
\end{tabular}


Table 5. Summary statistics comparing weighted average sulfur content (in weight percent) and potential $\mathrm{SO}_{2}$ emissions (in pounds of $\mathrm{SO}_{2}$ per million $\mathrm{British}$ thermal units) for 1983 through 1995 and 1996 through 2005.-Continued

[Data are shown by county. Data shown in columns 3 and 4 are from table 3; data shown in columns 8 and 9 are from table $4 . \mathrm{SO}_{2}$, sulfur dioxide; lb $\mathrm{SO}_{2} / \mathrm{MMBtu}$, pounds of sulfur dioxide per million British thermal units]

\begin{tabular}{|c|c|c|c|c|c|c|c|c|c|c|c|}
\hline State & County & $\begin{array}{l}\text { Weighted } \\
\text { average } \\
\text { sulfur for } \\
\text { 1983-1995 }\end{array}$ & $\begin{array}{c}\text { Weighted } \\
\text { average sulfur } \\
\text { for 1996-2005 }\end{array}$ & $\begin{array}{c}\text { Change in } \\
\text { weighted } \\
\text { average sulfur } \\
\text { (weight percent) }\end{array}$ & $\begin{array}{l}\text { Decrease or } \\
\text { increase }\end{array}$ & $\begin{array}{l}\text { Decrease } \\
\text { or increase } \\
\text { greater } \\
\text { than } 1\end{array}$ & $\begin{array}{c}\text { Weighted average } \\
\text { of potential } \mathrm{SO}_{2} \\
\text { emissions for } \\
1983-1995\end{array}$ & $\begin{array}{l}\text { Weighted aver- } \\
\text { age of potential } \\
\mathrm{SO}_{2} \text { emissions for } \\
1996-2005\end{array}$ & $\begin{array}{c}\text { Change in } \\
\text { weighted aver- } \\
\text { age of potential } \\
\mathrm{SO}_{2} \text { emissions } \\
\text { (lb SO } \mathrm{S}_{2} / \mathrm{MMBtu} \text { ) }\end{array}$ & $\begin{array}{l}\text { Decrease or } \\
\text { increase }\end{array}$ & $\begin{array}{l}\text { Decrease } \\
\text { or increase } \\
\text { greater } \\
\text { than } 1\end{array}$ \\
\hline W. Va. & Boone & 0.81 & 0.79 & -0.02 & Decrease & & 1.29 & 1.29 & 0.00 & No change & \\
\hline W. Va. & Braxton & 0.81 & No production & No production & No production & & 1.23 & No production & No production & No production & \\
\hline W. Va. & Brooke & 3.12 & 3.79 & 0.67 & Increase & & 5.14 & 6.15 & 1.01 & Increase & 1.01 \\
\hline W. Va. & Clay & 0.96 & 0.80 & -0.16 & Decrease & & 1.58 & 1.28 & -0.30 & Decrease & \\
\hline W. Va. & Fayette & 1.11 & 1.03 & -0.08 & Decrease & & 1.79 & 1.66 & -0.13 & Decrease & \\
\hline W. Va. & Gilmer & 2.40 & No production & No production & No production & & 3.70 & No production & No production & No production & \\
\hline W. Va. & Grant & 1.71 & 1.64 & -0.07 & Decrease & & 2.76 & 2.66 & -0.10 & Decrease & \\
\hline W. Va. & Greenbrier & 1.08 & 0.88 & -0.20 & Decrease & & 1.67 & 1.35 & -0.32 & Decrease & \\
\hline W. Va. & Harrison & 3.17 & 3.38 & 0.21 & Increase & & 4.88 & 5.41 & 0.53 & Increase & \\
\hline W. Va. & Kanawha & 0.83 & 0.76 & -0.07 & Decrease & & 1.38 & 1.25 & -0.13 & Decrease & \\
\hline W. Va. & Lewis & 2.86 & No production & No production & No production & & 4.44 & No production & No production & No production & \\
\hline W. Va. & Lincoln & 0.79 & 0.90 & 0.11 & Increase & & 1.33 & 1.43 & 0.10 & Increase & \\
\hline W. Va. & Logan & 0.72 & 0.73 & 0.01 & Increase & & 1.17 & 1.20 & 0.03 & Increase & \\
\hline W. Va. & McDowell & 0.81 & 0.78 & -0.03 & Decrease & & 1.24 & 1.16 & -0.08 & Decrease & \\
\hline W. Va. & Marion & 1.96 & 2.26 & 0.30 & Increase & & 3.15 & 3.56 & 0.41 & Increase & \\
\hline W. Va. & Marshall & 3.92 & 3.28 & -0.64 & Decrease & & 6.44 & 5.39 & -1.05 & Decrease & -1.05 \\
\hline W. Va. & Mason & 2.87 & 0.75 & -2.12 & Decrease & -2.12 & 4.89 & 1.14 & -3.75 & Decrease & -3.75 \\
\hline W. Va. & Mercer & 0.66 & 1.12 & 0.46 & Increase & & 1.03 & 1.72 & 0.69 & Increase & \\
\hline W. Va. & Mineral & 1.67 & 1.67 & 0.00 & No change & & 2.82 & 2.84 & 0.02 & Increase & \\
\hline W. Va. & Mingo & 0.76 & 0.76 & 0.00 & No change & & 1.21 & 1.23 & 0.02 & Increase & \\
\hline W. Va. & Monongalia & 2.35 & 2.22 & -0.13 & Decrease & & 3.66 & 3.47 & -0.19 & Decrease & \\
\hline W. Va. & Nicholas & 0.90 & 0.92 & 0.02 & Increase & & 1.41 & 1.50 & 0.09 & Increase & \\
\hline W. Va. & Ohio & 3.16 & 4.17 & 1.01 & Increase & 1.01 & 5.33 & 6.93 & 1.60 & Increase & 1.60 \\
\hline W. Va. & Preston & 1.62 & 1.53 & -0.09 & Decrease & & 2.58 & 2.35 & -0.23 & Decrease & \\
\hline W. Va. & Raleigh & 0.86 & 1.11 & 0.25 & Increase & & 1.28 & 1.84 & 0.56 & Increase & \\
\hline
\end{tabular}


Table 5. Summary statistics comparing weighted average sulfur content (in weight percent) and potential $\mathrm{SO}_{2}$ emissions (in pounds of $\mathrm{SO}_{2}$ per million $\mathrm{British}$ thermal units) for 1983 through 1995 and 1996 through 2005.-Continued

[Data are shown by county. Data shown in columns 3 and 4 are from table 3; data shown in columns 8 and 9 are from table $4 . \mathrm{SO}_{2}$, sulfur dioxide; $1 \mathrm{~b} \mathrm{SO} / \mathrm{MMBtu}_{2}$ pounds of sulfur dioxide per million British thermal units]

\begin{tabular}{|c|c|c|c|c|c|c|c|c|c|c|c|}
\hline State & County & $\begin{array}{l}\text { Weighted } \\
\text { average } \\
\text { sulfur for } \\
1983-1995\end{array}$ & $\begin{array}{l}\text { Weighted } \\
\text { average sulfur } \\
\text { for 1996-2005 }\end{array}$ & $\begin{array}{l}\text { Change in } \\
\text { weighted } \\
\text { average sulfur } \\
\text { (weight percent) }\end{array}$ & $\begin{array}{l}\text { Decrease or } \\
\text { increase }\end{array}$ & $\begin{array}{l}\text { Decrease } \\
\text { or increase } \\
\text { greater } \\
\text { than } 1\end{array}$ & $\begin{array}{c}\text { Weighted average } \\
\text { of potential } \mathrm{SO}_{2} \\
\text { emissions for } \\
1983-1995\end{array}$ & $\begin{array}{l}\text { Weighted aver- } \\
\text { age of potential } \\
\mathrm{SO}_{2} \text { emissions for } \\
1996-2005\end{array}$ & $\begin{array}{c}\text { Change in } \\
\text { weighted aver- } \\
\text { age of potential } \\
\mathrm{SO}_{2} \text { emissions } \\
\left.\text { (lb SO} \mathrm{S}_{2} / \mathrm{MMBtu}\right)\end{array}$ & $\begin{array}{l}\text { Decrease or } \\
\text { increase }\end{array}$ & $\begin{array}{l}\text { Decrease } \\
\text { or increase } \\
\text { greater } \\
\text { than } 1\end{array}$ \\
\hline W. Va. & Randolph & 1.18 & 0.72 & -0.46 & Decrease & & 1.85 & 1.13 & -0.72 & Decrease & \\
\hline W. Va. & Taylor & 1.52 & No production & No production & No production & & 2.51 & No production & No production & No production & \\
\hline W. Va. & Tucker & 1.77 & No production & No production & No production & & 3.05 & No production & No production & No production & \\
\hline W. Va. & Upshur & 1.63 & 1.80 & 0.17 & Increase & & 2.51 & 2.84 & 0.33 & Increase & \\
\hline W. Va. & Wayne & 0.86 & 0.80 & -0.06 & Decrease & & 1.44 & 1.33 & -0.11 & Decrease & \\
\hline W. Va. & Webster & 0.84 & 0.97 & 0.13 & Increase & & 1.30 & 1.55 & 0.25 & Increase & \\
\hline \multirow[t]{12}{*}{ W. Va. } & Wyoming & 0.92 & 0.84 & -0.08 & Decrease & & 1.35 & 1.30 & -0.05 & Decrease & \\
\hline & & & Total records & 157 & 100.0 percent & & & Total records & 157 & 100.0 percent & \\
\hline & & No produ & ction 1996-2005 & 30 & 19.1 percent & & No pr & roduction 1996-2005 & 30 & 19.1 percent & \\
\hline & & Prod & luced 1996-2005 & 127 & 80.9 percent & & & Produced 1996-2005 & 127 & 80.9 percent & \\
\hline & & howed increas & ed percent sulfur & 53 & 41.7 percent & & Showed increa & ase in $\mathrm{lb} \mathrm{SO}_{2} / \mathrm{MMBtu}$ & 58 & 45.7 percent & \\
\hline & & howed decreas & ed percent sulfur & 71 & 55.9 percent & & Showed decrea & ase in $\mathrm{lb} \mathrm{SO}_{2} / \mathrm{MMBtu}$ & 68 & 53.5 percent & \\
\hline & Sho & wed no change & in percent sulfur & 3 & 2.4 percent & & Showed no chan & ge in $\mathrm{lb} \mathrm{SO}_{2} / \mathrm{MMBtu}$ & 1 & 0.8 percent & \\
\hline & & Showed increa & ase greater than 1 & 5 & 4.0 percent & & Showed in & acrease greater than 1 & 12 & 9.5 percent & \\
\hline & & Showed decr & rease less than -1 & 3 & 2.4 percent & & Showed & decrease less than -1 & 11 & 8.7 percent & \\
\hline & & nowed change 1 & between -1 and 1 & 116 & 93.5 percent & & Showed chan & nge between -1 and 1 & 103 & 81.7 percent & \\
\hline & & & aximum increase & 4.21 & & & & Maximum increase & 8.18 & & \\
\hline & & & aximum decrease & -2.12 & & & & Maximum decrease & -3.75 & & \\
\hline
\end{tabular}

